Portland State University

PDXScholar

Winter 2-12-2016

\title{
One-Dimensional Nanostructure and Sensing Applications: Tin Dioxide Nanowires and Carbon Nanotubes
}

Hoang Anh Tran

Portland State University

Follow this and additional works at: https://pdxscholar.library.pdx.edu/open_access_etds

Part of the Chemistry Commons, Materials Science and Engineering Commons, and the Nanoscience and Nanotechnology Commons

Let us know how access to this document benefits you.

\section{Recommended Citation}

Tran, Hoang Anh, "One-Dimensional Nanostructure and Sensing Applications: Tin Dioxide Nanowires and Carbon Nanotubes" (2016). Dissertations and Theses. Paper 2689.

https://doi.org/10.15760/etd.2685

This Dissertation is brought to you for free and open access. It has been accepted for inclusion in Dissertations and Theses by an authorized administrator of PDXScholar. Please contact us if we can make this document more accessible: pdxscholar@pdx.edu. 
One-Dimensional Nanostructure and Sensing Applications:

Tin Dioxide Nanowires and Carbon Nanotubes

by

Hoang Anh Tran

A dissertation submitted in partial fulfillment of the requirements for the degree of

Doctor of Philosophy

in

Chemistry

Dissertation Committee:

Shankar Rananavare, Chair

Carl Wamser

Albert Benight

Raj Solanki

Erik Sanchez

James Blackwell

Portland State University

2016 


\begin{abstract}
The key challenge for a nanomaterial based sensor is how to synthesize in bulk quantity and fabricate an actual device with insightful understanding of operational mechanisms during performance. I report here effective, controllable methods that exploit the concepts of the "green approach" to synthesize two different one-dimensional nanostructures, including tin oxide nanowires and carbon nanotubes. The syntheses are followed by product characterization and sensing device fabrications as well as sensor performance understanding at the molecular level. Sensor-analyte response and recovery kinetics are also presented.
\end{abstract}

The first part of the thesis describes bulk-scale synthesis and characterization of tin oxide nanowires by the molten salt synthetic method and the nanowire doping with antimony (n-types) and lithium. The work builds on the success of using n-doped $\mathrm{SnO}_{2}$ nanoparticles to selectively detect chlorine gas at room temperature. Replacing n-doped nanoparticles with n-doped nanowires reduces the number of inter-particle electron hops between sensing electrodes. The nanowire based sensors show unprecedented $5 \mathrm{ppb}$ detectability of corrosive $\mathrm{Cl}_{2}$ gas concentration in air. At the higher range, $10 \mathrm{ppm}$ of $\mathrm{Cl}_{2}$ gas leads to a 250 fold increase in the device resistance. During sensor recovery, FT-IR studies show that dichlorine monoxide $\left(\mathrm{Cl}_{2} \mathrm{O}\right)$ and chlorine dioxide $\left(\mathrm{ClO}_{2}\right)$ are the desorbing species. Long term stability of devices is affected by lattice oxygen vacancies replaced by chlorine atoms.

Bulk-scale synthesis of multiwall carbon nanotube (MWCNTs) was achieved by a novel inexpensive synthetic method. The green chemistry method uses the non-toxic 
and easy to handle solid carbon source naphthalene. The synthesis is carried out by simply heating naphthalene and organometallic precursors as catalysts in a sealed glass tube. Synthesis at $610^{\circ} \mathrm{C}$ leads to MWCNTs of $50 \mathrm{~nm}$ diameter and lengths exceeding well over microns. MWCNT doping is attempted with nitrogen (n-type) and boron (ptype) precursors. Palladium nanoparticles decorated on as-synthesized MWCNTs are employed for specific detection of explosive hydrogen gas with concentrations far below the explosive concentration limits. During performance, the sensor exhibits abnormal response behaviors at hydrogen gas concentrations higher than $1 \%$. A model of charge carrier inversion, brought about by reduction of MWCNT by hydrogen molecules dissociated by Pd nanoparticles is proposed. 
To my beloved Dad, I miss you here on Earth.

This dissertation is dedicated to you. 


\section{Acknowledgements}

It gives me a pleasure to gratefully and sincerely thank my advisor, Professor S.B Rananavare. I have been extremely fortunate to have an exceptional advisor who gave me invaluable guidance, constant inspiration and continued support during my academic career at Portland State University. All that has been achieved in this thesis was made possible by him.

I am also grateful to Professors Carl Wamser, Albert Benight, Raj Solanki, and Erik Sanchez for their time and invaluable inputs in my dissertation committee. I also would like to express my gratitude to Dr. James Blackwell for not only serving in my dissertation committee but also giving me a chance to work with different collaborators and sponsoring me through Intel's funding.

I am indebted to Professor Andrea Goforth for photoluminescence analysis, Professor Andres La Rosa for the usage of the Raman system. Especially, I am thankful to Dr. Daner Abdulla for his intellectual help on Raman spectroscopy as well as uncountable advice on my thesis. Many thanks go to Dr. Joo Chan and Dr. Allen Chapadraza for the treasure of knowledge I had inherited when I was still an undergraduate student. I also would like to thank Professor Rananavare's research group Ryan Lerud, Srikar Rao, Kayode Morakinyo, Sayan just to mention a few, for their support and help during my PhD studies.

Most importantly, none of this would have been possible without the support, love patience of my family. I would like to take this opportunity to thank my uncle Le's 
family, who has given me all the supports and foundation in this country. Without them I would not be the person that I am today.

To my wife Leyna, I know you suffer from my imperfections and my shortcomings largely during the challenging moments. Your sweetness, your kindness, the beauty of your heart and your thoughtfulness never cease to amaze me. You are the motivation of my life.

To my mom, I know I owe you everything that I am. I could not ask for a better mom than you. Thank-you mom for your patience, your wisdom and most important, your love. 
Table of Contents

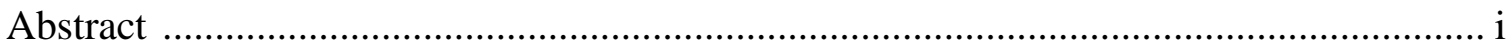

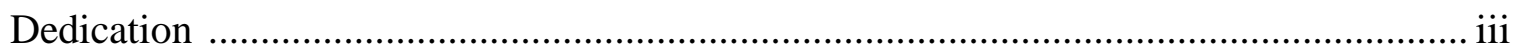

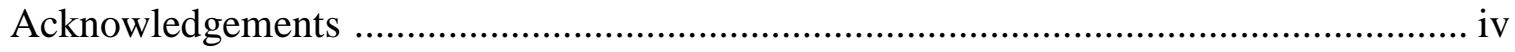

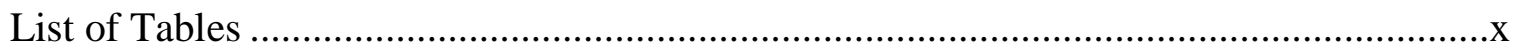

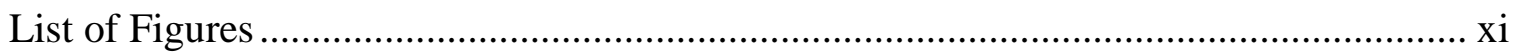

CHAPTER 1. SENSOR BASED ON ONE DIMENSIONAL NANOMATERIALS........1

1.1 General Introduction Nanomaterials .............................................................

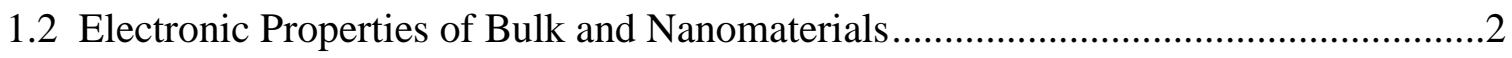

1.3 One Dimensional Nanomaterials for Sensing Applications ..................................6

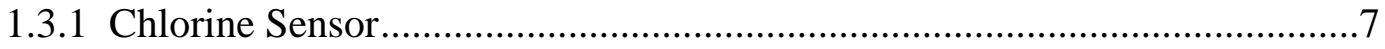

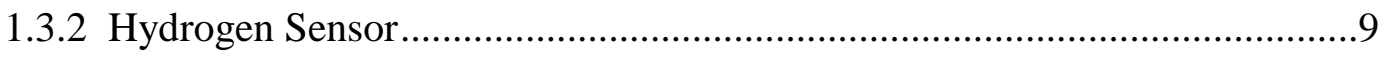

CHAPTER 2. INSTRUMENTATION, DEVICE CHARACTERIZATION AND

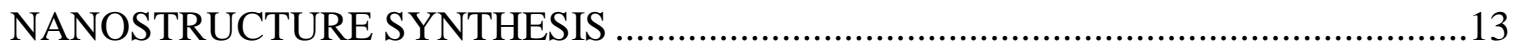

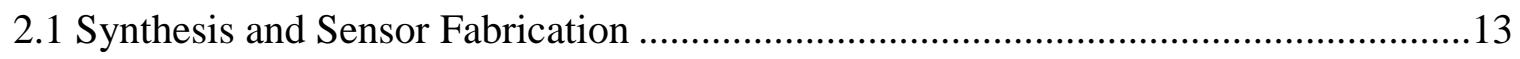

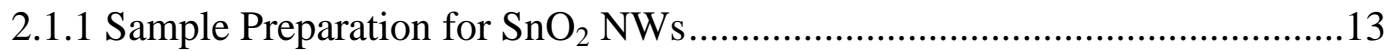

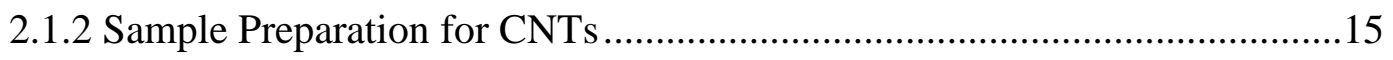

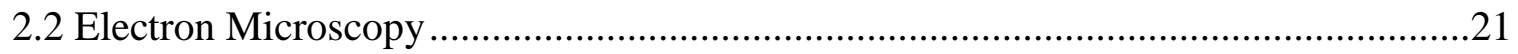

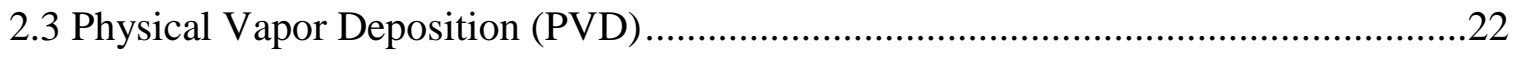

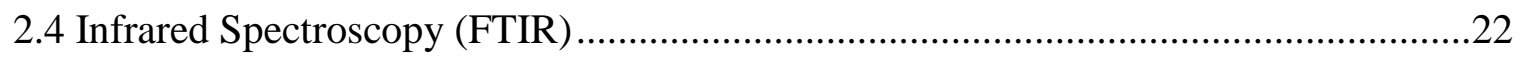

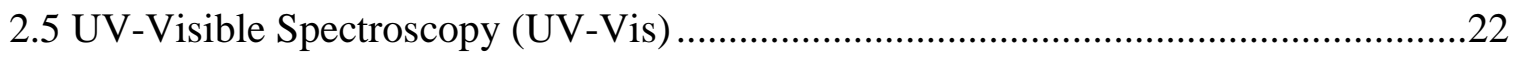

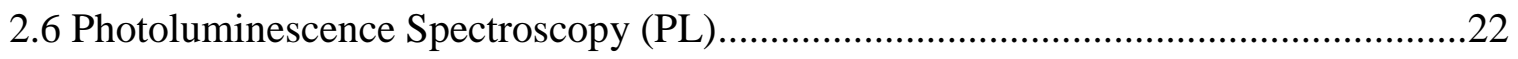




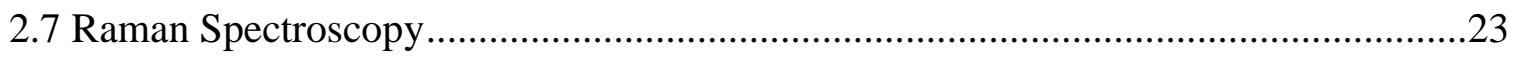

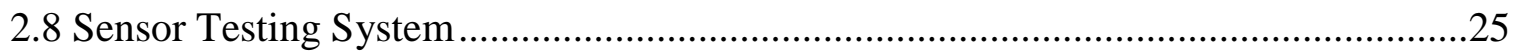

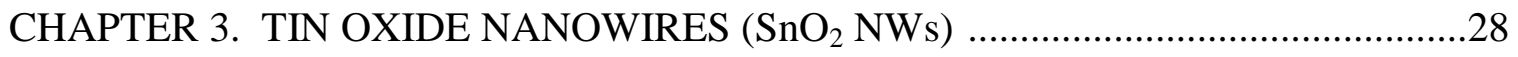

3.1 Background and Significance ………..................................................................28

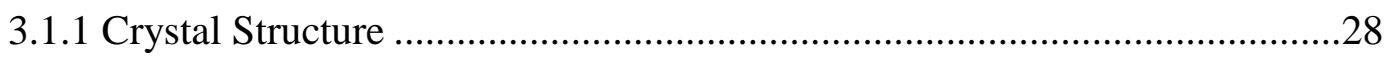

3.1.2 Stoichiometry, Defect Structure and Conductivity ....................................29

3.1.3 Raman Active Vibrational Modes of $\mathrm{SnO}_{2}$ Rutile Structure .........................31

3.1.4 Growth of $\mathrm{SnO}_{2} \mathrm{NWs}$

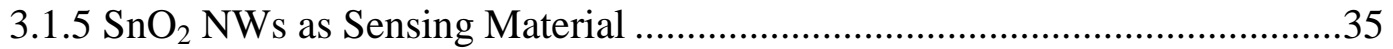

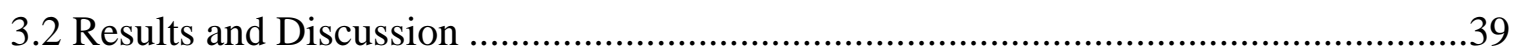

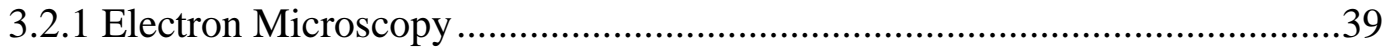

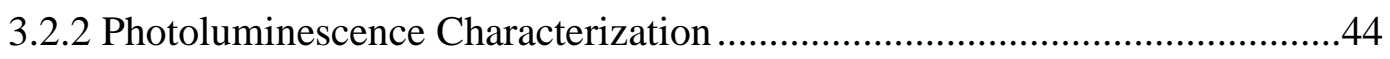

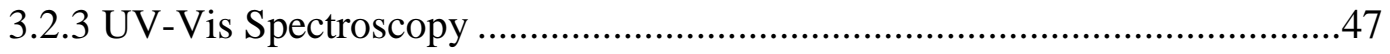

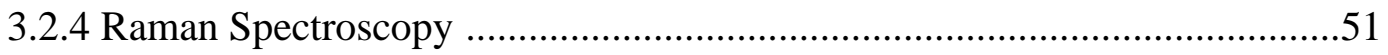

3.2.5 Electrical Properties of Thick Film Using n-doped $\mathrm{SnO}_{2} \mathrm{NWs}$...................53

3.3 Sensing Application: Chlorine Resistive Gas Sensor Based on n-doped $\mathrm{SnO}_{2} \mathrm{NWs}_{55}$

3.3.1 Sensor Response, Response Time and Recovery Time .................................55

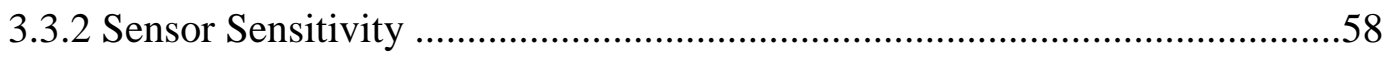

3.3.3 Sensor Stability and Understanding Sensing Mechanism ..........................63

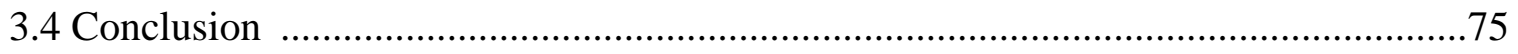

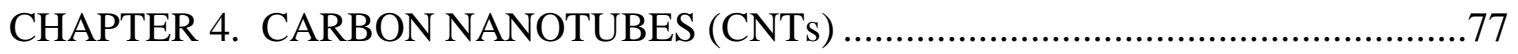

4.1 Background and Significance …………………….................................................77 
4.1.1 Electronic Properties

4.1.2 Growth of CNTs .79

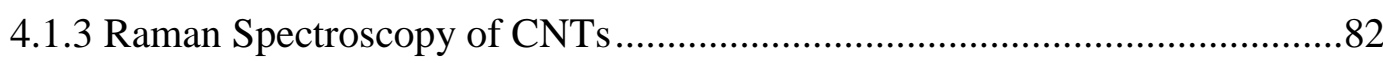

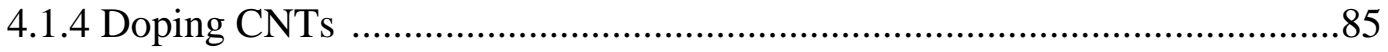

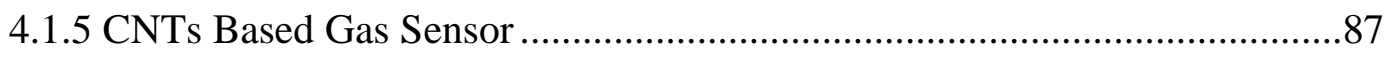

4.2 Results and Discussion ...................................................................................

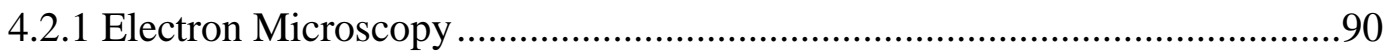

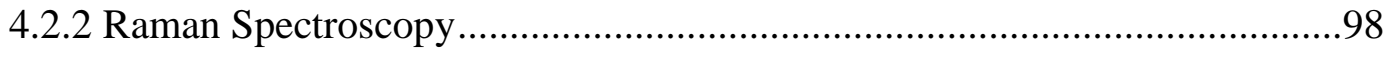

4.2.3 Palladium Nanoparticles Attached MWCNTs for Hydrogen Sensor .........103

4.3 Sensing Application: Hydrogen Gas Sensor Based on Pd NPs Attached MWCNTs 107

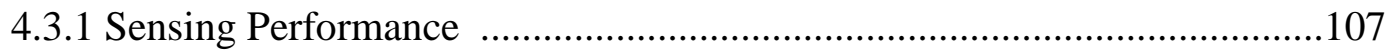

4.3.2 Understanding Sensing Mechanism.........................................................113

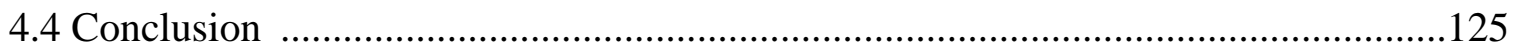

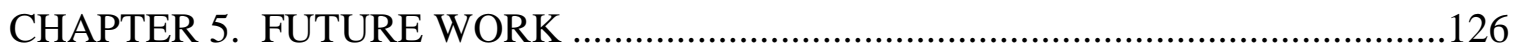

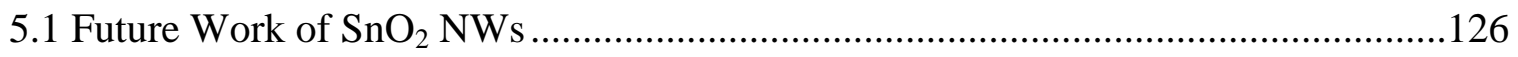

5.2 Future Work of CNTs ………………………...............................................127

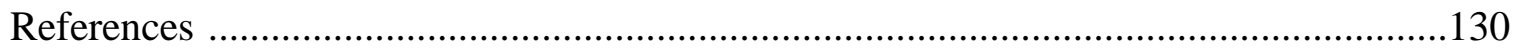

Appendices

Appendix A. Chemical Reagents ...........................................................................150

Appendix B. Characterization of $\mathrm{SnO}_{2} \mathrm{NWs}$ at Different Synthetic Conditions …........151

Appendix C. Synthesis and Characterization of Heterojuctions of Silicon Nanowires (SiNWs) and Carbon Nanotubes (CNTs) ………….............................................158 
Appendix D. Negative Differential Resistance Observed from Pd NPs - CNTs .161 
List of Tables

Table 1: A brief summary of nanomaterial based chlorine sensors ............................... 8

Table 2: A brief summary of metal oxide based hydrogen sensors .............................. 10

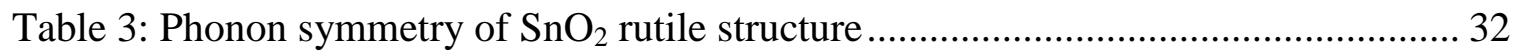

Table 4: The effect of synthetic conditions on $\mathrm{SnO}_{2}$ NWs morphology ........................ 43

Table 5: Optical bandgap determined from UV absorption edge and PL...................... 50

Table 6: Raman data analysis of different $\mathrm{SnO}_{2}$ samples ........................................ 52

Table 7: Kinetic analysis of response curves during the exposure process. .................... 70

Table 8: Kinetic analysis of response curves during the recovery process..................... 72

Table 9: IR observation of dichlorine monoxide $\left(\mathrm{Cl}_{2} \mathrm{O}\right)$ and chlorine dioxide $\left(\mathrm{ClO}_{2}\right) \ldots . .74$

Table 10: The non-linear least squares analysis of D and G bands from Raman

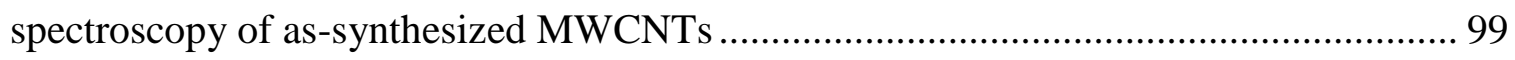

Table 11: G' bands from Raman spectroscopy of as-synthesized MWCNTs ............... 101

Table 12: Summary kinetic constants from fitted response curves ........................... 115

Table 13: Summary of kinetic constants from fitted recovery curves ......................... 118 


\section{List of Figures}

Figure 1: Illustration of band diagram for an intrinsic semiconductor.

Figure 2: The density of states $\mathrm{D}(\mathrm{E})$ (left) for a 3D semiconductor and Fermi-Dirac distribution $\mathrm{f}(\mathrm{E})$ (right) at different temperatures for intrinsic semiconductor................. 4

Figure 3: Schematic showing density of state of low dimensional nanostructures. .......... 5

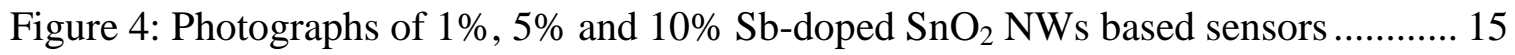

Figure 5: An optical image of duo-chamber glass reaction vessel for the synthesis of

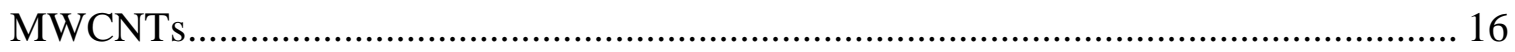

Figure 6: Schematic illustration of CNTs synthesis from three different synthetic

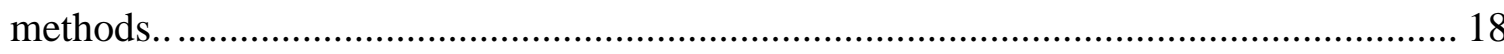

Figure 7: The photograph of the custom-built CNT synthesis system housed inside a tube

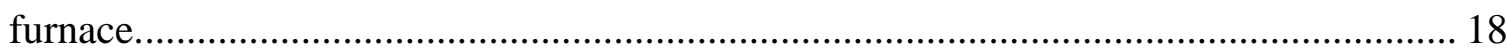

Figure 8: Molecular structure of doping agents for CNTs...................................... 19

Figure 9: A photograph of fabricated CNT based sensor using method C..................... 21

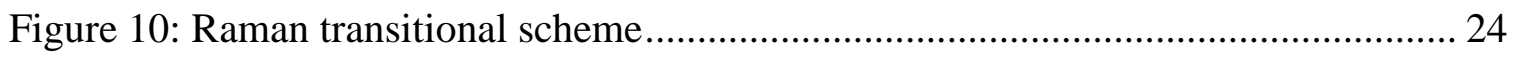

Figure 11: Schematic showing the home-built Raman system with major components. . 25

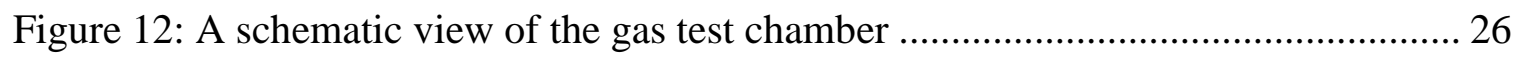

Figure 13: Schematic of gas diluting system (left) and the photograph of home-built

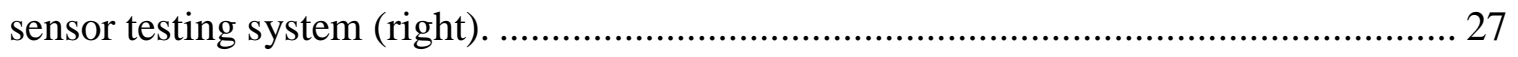

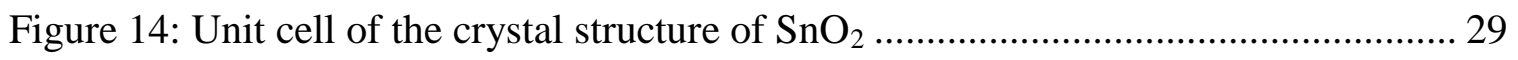

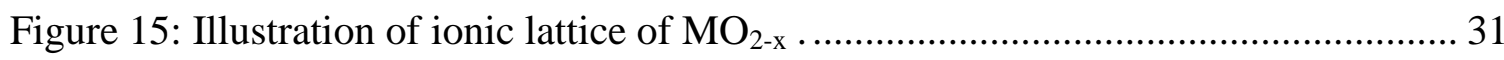

Figure 16: Raman active vibrational modes of $\mathrm{SnO}_{2}$ rutile structure ........................... 33 
Figure 17: A. Energy levels of oxidizing and reducing gases in relation to band edges of

$\mathrm{SnO}_{2}$. B. Location and position $\mathrm{Sb}$ reduction potential. .......................................... 37

Figure 18: Schematic diagrams of porous sensing layers...................................... 38

Figure 19: Optical images of undoped (left) and Sb doped NWs (right) ....................... 39

Figure 20: SEM images of undoped (left) and Sb doped NWs (right) ....................... 41

Figure 21: TEM images of n-doped $\mathrm{SnO}_{2} \mathrm{NWs}$ with low (left) and high magnifications along with electron diffraction in the inset (right)............................................ 41

Figure 22: Optical image and SEM image of Li-doped $\mathrm{SnO}_{2} \mathrm{NWs}$............................ 42

Figure 23: SEM image of Li-doped $\mathrm{SnO}_{2} \mathrm{NWs}$ synthesized at $600^{\circ} \mathrm{C}$ and $700^{\circ} \mathrm{C} \ldots \ldots . .43$

Figure 24: A) HR-TEM of $\mathrm{SnO}_{2-\mathrm{x}}$ coated $\mathrm{Sn}$ NP precursors and B) TEM of Li-doped

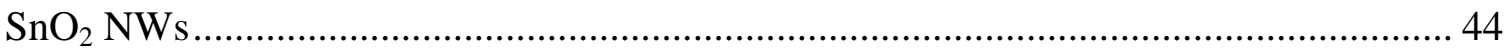

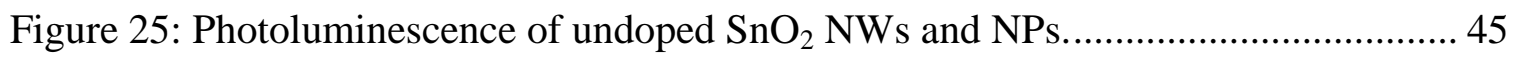

Figure 26: Photoluminescence of undoped and n-doped $\mathrm{SnO}_{2} \mathrm{NWs}^{2}$.......................... 46

Figure 27: Photoluminescence of undoped, 5\% n-doped and 5\% Li-doped................... 47

Figure 28: UV-Vis spectra of different $\mathrm{SnO}_{2} \mathrm{NW}$ samples: undoped, 5\% n-doped and 5\%

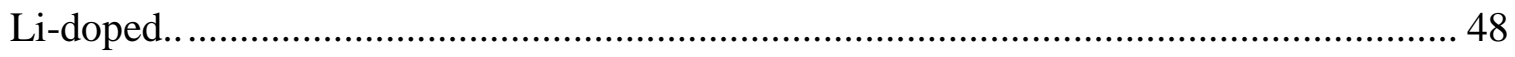

Figure 29: Determination of optical bandgap from the UV absorption edge................. 49

Figure 30: Raman spectrum of $\mathrm{SnO}_{2}$ NWs samples............................................. 51

Figure 31: Illustration of curve fitting of $2 \%$ n-doped $\mathrm{SnO}_{2} \mathrm{NWs}$ sample....................... 52

Figure 32: I-V curves of different dopant concentrations of n-doped NW based sensors 54

Figure 33: The sensor resistance (A) and recovery time (B) at six different $\mathrm{Cl}_{2}$

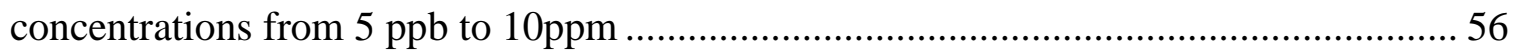


Figure 34: Response and recovery time (minute) as a function of temperature $\left({ }^{\circ} \mathrm{K}\right) \ldots \ldots . .56$

Figure 35: Response as a function of chlorine concentration at room temperature.......... 59

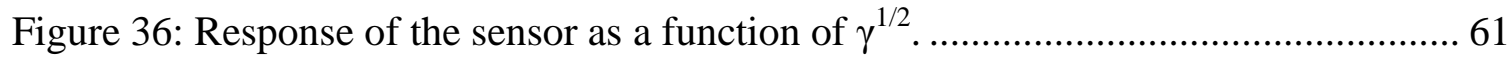

Figure 37: Response of the sensor as a function of doping concentration....................... 62

Figure 38: Schematic diagram of the porous sensing layer composed from nanowires... 65

Figure 39: Response of the sensor from 4 continuous cycles of exposure and recovery. 65

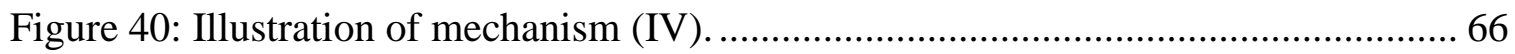

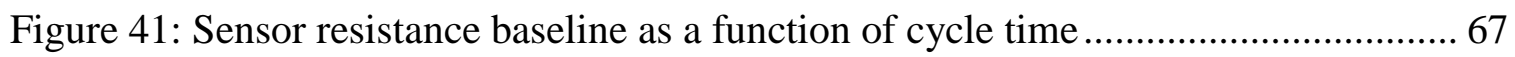

Figure 42: Sensor response curves after storing in high $\mathrm{Cl}_{2}$ concentration ................... 68

Figure 43: An example of fitting sensor response resistance as a function of time.......... 70

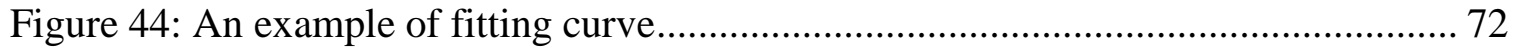

Figure 45: Infrared spectrum composed from blank sample (A), mixture of chorine and

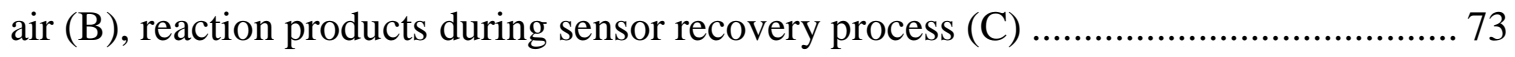

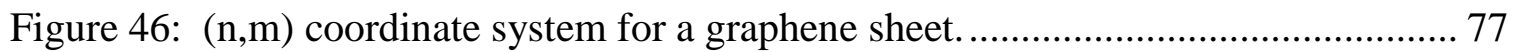

Figure 47: Schematic diagram of a DC-arc discharge system for CNT synthesis........... 80

Figure 48: Schematic diagram of a laser ablation system to synthesize CNTs .............. 81

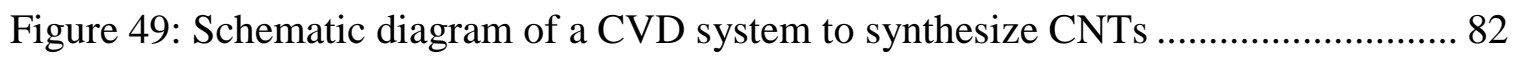

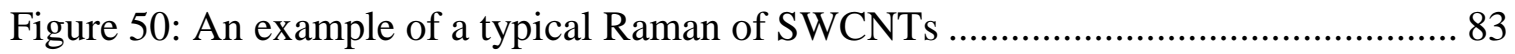

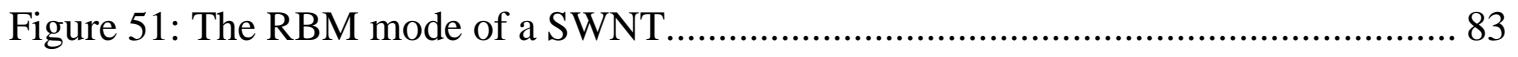

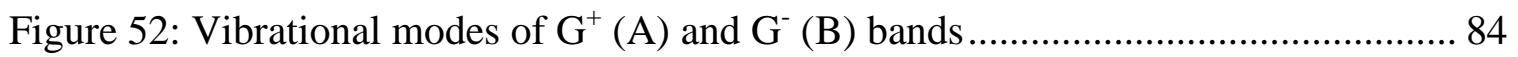

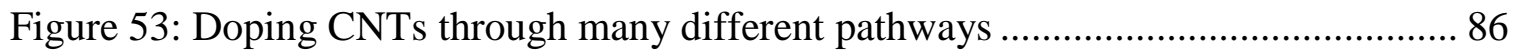

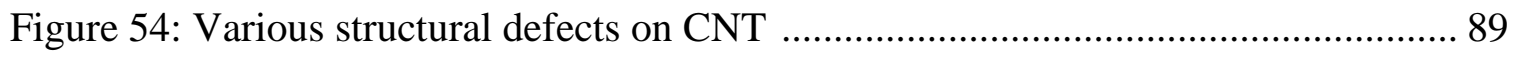


Figure 55: An example of $\mathrm{NH}_{3}$ molecule adsorption on defective CNT 89

Figure 56: Formation of Ni nanoparticle catalysts after annealing 5nm and 20nm thick Ni thin films at $680^{\circ} \mathrm{C}$. 90

Figure 57: Method A for the synthesis of CNTs at $590^{\circ} \mathrm{C}, 600^{\circ} \mathrm{C}, 610^{\circ} \mathrm{C}$ and $620^{\circ} \mathrm{C} \ldots \ldots 91$ Figure 58: HRTEM of CNT synthesized at $600^{\circ} \mathrm{C}$ in $2 \mathrm{hrs}$ (top) and EDX of catalyst (bottom) and STEM of a CNT. 94

Figure 59: Method B for the synthesis of CNTs in uncontrolled manner at $590^{\circ} \mathrm{C}, 600^{\circ} \mathrm{C}$, $610^{\circ} \mathrm{C}$ and $620^{\circ} \mathrm{C}$ 95

Figure 60: Optical images of method C for the synthesis of CNTs ferrocene (top) and

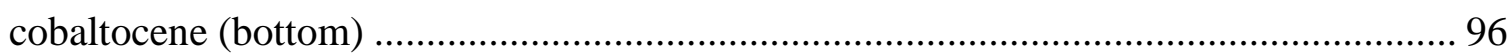

Figure 61: SEM images of CNTs by method C.................................................... 96

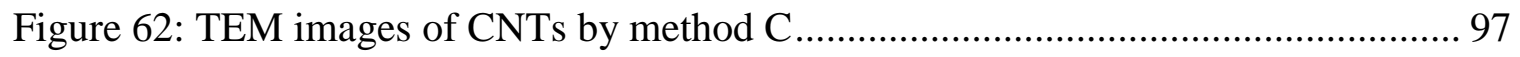

Figure 63: Raman spectroscopy of as-synthesized MWCNTs ................................. 98

Figure 64: An example of fitting using the undoped CNT sample.............................. 99

Figure 65: Raman Spectroscopy of as-synthesized MWCNTs.................................. 101

Figure 66: The band intensity ratios of D/G and G'/G of p-doped CNTs (left) and ndoped CNTs (right)

Figure 67: SEM images of commercial CNTs and palladium nanoparticle decorated CNTs. 104

Figure 68: Illustrative concept for the Decoration of CNTs with Pd Nanoparticles ...... 105 Figure 69: SEM of thin film of CNTs deposited on the wall of glass tube before and after the attachment of Pd nanoparticles. 106 
Figure 70: Low and high magnification TEM images of as-synthesized CNTs decorated with Pd nanoparticles 106

Figure 71: Response of testing sensor upon the exposure of $4 \% \mathrm{H}_{2}$ with the inset showing the initial stage of $\mathrm{H}_{2}$ exposure with the flow rate of $1 \mathrm{~L} /$ minute 107 Figure 72: Response of Pd nanoparticles attached as-synthesized CNTs sensor to different $\mathrm{H}_{2}$ concentrations from 10ppm to $4 \%(40,000 \mathrm{ppm})$................................ 109 Figure 73: An example of fitted curve to $10 \mathrm{ppm} \mathrm{H}_{2}$ 110 Figure 74: Slope of the response curve as a function of $\mathrm{H}_{2}$ concentrations.

Figure 75: Abnormal behaviors of sensor response and recovery at high $\mathrm{H}_{2}$ concentrations.

Figure 76: Electrochemical energy level diagram of hydrogen, palladium and oxygen in relation to the Fermi level of CNTs

Figure 77: Illustration of the response mechanism of Pd Nps decorated CNTs upon the exposure with $\mathrm{H}_{2}$

Figure 78: Sensor response at 10ppm $\mathrm{H}_{2}$ concentration . 115

Figure 79: $\mathrm{H}_{2}$ molecular dissociation constant (left) and electrons transferred to nanotube constant (right) change as a function of $\mathrm{H}_{2}$ concentration 116

Figure 80: Sensor recovery after $10 \mathrm{ppm} \mathrm{H}_{2}$ exposure 117 Figure 81: a) 5 stages of charge carrier inversion process b) Time dependence of excess hole and electron concentration in CNT. 119 Figure 82: Mechanism of 5 stages of charge carrier inversion process (left) and electronic band diagram of sensing process (right) 121 
Figure 83: Kinetic of charge carrier inversion and slow recovery using diluted air...... 123

Figure 84: Response of n-doped $\mathrm{SnO}_{2} / \mathrm{Pd}$ (right) sensors to different hydrogen

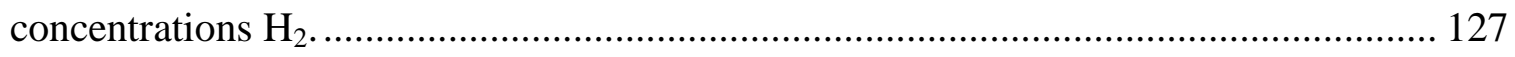

Figure 85: The sketch illustrating experiment for future study of NDR-like behavior using

two individuals Pd Np attached CNTs........................................................ 128 


\section{CHAPTER 1. SENSOR BASED ON ONE DIMENSIONAL NANOMATERIALS}

\subsection{General Introduction Nanomaterials}

Nanomaterials are cornerstone of nanoscience and nanotechnology. In the past few decades, a broad and interdisciplinary area of nanostructure research and development has been growing explosively due to their novel properties and their emerging new applications. However, nanomaterials have a long scientific history, which began after the first research report by Michael Faraday in 1857 with the introduction of colloidal gold nanoparticle (NP) synthesis [1]. One of the major industrial applications of nanomaterials emerged in the early 1940s in the United States of America and Germany with the replacement of ultrafine carbon black by precipitated and fumed silica nanoparticles for rubber reinforcements [2]. Another prime example is the investigation of nanostructural aspects of catalysts over the last 150 years [3]. Today, nanophase engineering has expanded in a rapidly growing number of structural and functional materials, both inorganic and organic, allowing for the manipulation of mechanical, catalytic, electric, magnetic, optical and electronic functions [3].

Nanomaterials are defined as the materials whose structures have at least one dimension less than $100 \mathrm{~nm}$. Depending on how many dimensions are less than $100 \mathrm{~nm}$, nanomaterials are classified into three different categories: zero (0D, nanoparticles), one (1D, nanowires /nanotubes), and two dimensional (2D, nanofilms) nanostructures [3]. The size and dimension of nanomaterials lead to some remarkable properties that may be significantly different from the properties of bulk materials. Some such peculiar properties have been well-known and studied, but there is surely a plethora more to be 
discovered. Many physical properties of nanomaterials are related to the increased surface area and quantum confinement effects [4]. Compared to their bulk counterparts, nanomaterials have a much greater surface area to volume ratio, which can provide greater chemical reactivity and can provide better mechanical strength in composites. Quantum effects at the nanoscale determine the material's properties and characteristics, leading to novel optical (bandgap engineering e.g., CdSe, Si NPs), electrical and magnetic characteristics different from their conventional bulk forms. Currently, intense worldwide research has been focused on the synthesis, characterization, assembly and manipulation of nanostructural blocks for their device applications [5].

In this thesis, sensors based on one dimensional nanomaterials are developed that exploit their unusual electrical properties, especially conductivity, which is reviewed in the following section.

\subsection{Electronic Properties of Bulk and Nanomaterials}

A fundamental electronic property of materials is its conductivity which varies dramatically for metals, semiconductors and insulators. The conductivity $\sigma_{\text {tot }}$ of a general semiconductor crystal can be described as the sum due to electronic (ionic $\sigma_{i o n}$, electron $\sigma_{\mathrm{e}}$ and hole $\sigma_{\mathrm{p}}$ ) carriers if the associated conduction processes are considered independent [6]:

$$
\sigma_{\text {tot }}=\sigma_{\varepsilon}+\sigma_{p}+\sum \sigma_{i o n, i} \approx \sigma_{e}+\sigma_{p}=n \cdot \mu_{e} \cdot e+p \cdot \mu_{p} . e
$$

Where $n, p$ and $e$ are electron concentration, hole concentration and elementary charge $\left(1.6 \times 10^{-19}\right.$ coulombs), respectively. $\mu_{i}$ denotes the mobility of carrier i. In 
chemical sensing carrier concentrations or mobilities of the carriers are modulated by interaction with analyte.

Bulk material:

The resistance, an experimentally measured quantity of a homogeneous bulk material, can be related to its bulk conductivity $\sigma_{b}$, and geometric features. Geometrical features that affect resistance are its length $l$ and cross section area $A$. Thus for a bulk semiconductor:

$$
R_{b}=\frac{l}{\sigma_{b} \cdot A} \text { with } \sigma_{b}=\sigma_{e}+\sigma_{p}=n \cdot \mu_{e} \cdot e+p \cdot \mu_{p} \cdot e
$$

The charge carrier concentrations of electrons $(n)$ and holes $(p)$ for an intrinsic semiconductor can be calculated [6]:

$$
n=\int_{E_{c}}^{\infty} D(E) f(E) d E \quad ; \quad p=\int_{-\infty}^{E_{v}} D(E)(1-f(E)) d E
$$

With the density of states $D(E)$ and Fermi-Dirac distribution $f(E)$ are defined as [7]:

$$
D(E)=\frac{1}{2 \pi^{2}}\left(\frac{2 m_{e}}{\hbar^{2}}\right)^{\frac{3}{2}}\left(E-E_{C}\right)^{\frac{1}{2}} ; f(E)=\frac{1}{1+\exp \left(\frac{E-E_{F}}{k T}\right)}
$$

Where $E_{C}, E_{V}$ and $E_{F}$ are conduction band and valence band edges and Fermi energy level, respectively. $k, m_{e}, h$ are Boltzmann constant, mass of carrier, and Planck's constant respectively. These band edges are depicted along with band gap $E_{g}$ in Figure 1: 


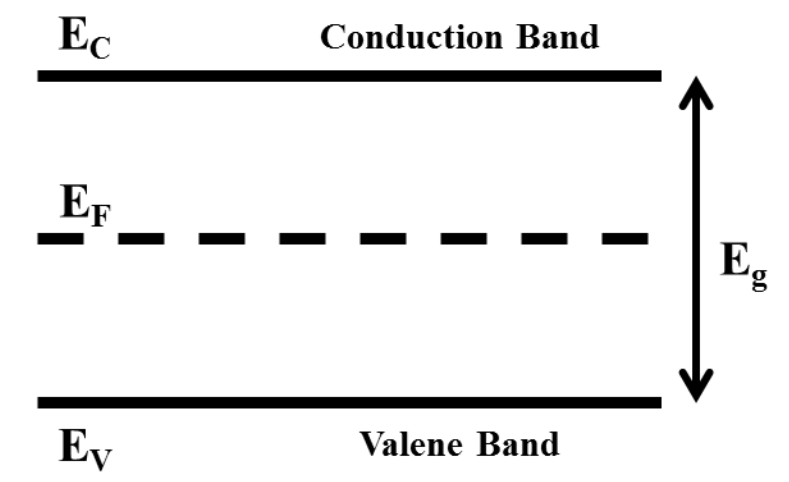

Figure 1: Illustration of band diagram for an intrinsic semiconductor.

The variation of density of state for a bulk 3D semiconductor and the Fermi functions for a bulk semiconductor are illustrated below in Figure 2:
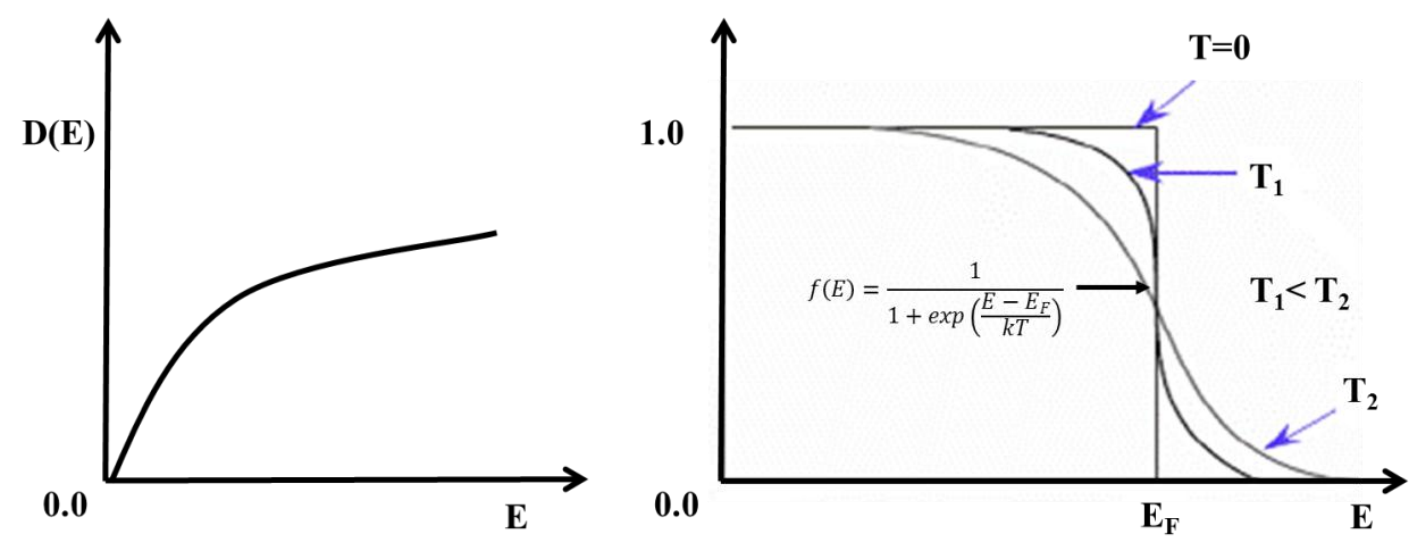

Figure 2: The density of states $\mathrm{D}(\mathrm{E})$ (left) for a 3D semiconductor and Fermi-Dirac distribution $\mathrm{f}(\mathrm{E})$ (right) at different temperatures for intrinsic semiconductor [8].

Low-dimensional nanostructures: 0,1 , and 2 dimensions:

In cases of low-dimensional nanostructures, the conductivity is different from its counterpart in bulk 3D material. It can also be defined as equation (1.1), and the charge carrier concentrations electrons $(n)$ and holes $(p)$ can be calculated by equation (1.3). The Fermi-Dirac distribution function $f(E)$ stays the same, but the density of states $D(E)$ depends on the dimensionality. 
A general method to calculate the density of states in energy and wavevector space (E versus $\mathrm{k}$ space) of these low-dimensional nanostructures follows equation (1.5) with the representative density of states for 0,1 and 2 dimensional nanostructures are illustrated in Figure 3:

$$
D(E)=2 \frac{V}{(2 \pi)^{3}} \frac{1}{\frac{d E_{k}}{d \vec{k}}}
$$

Where $\mathrm{V}$ is the volume of a spherical shell in $\mathrm{k}$ space

In one-dimensional nanostructure the density of state is calculated as [8]:

$$
D(E)=\frac{L}{2 \pi}\left(\frac{\hbar}{2 m^{*}}\right) \frac{1}{\sqrt{E}} \equiv \frac{L}{2 \pi}\left(\frac{\hbar}{2 m^{*}}\right) \frac{1}{\sqrt{E-E_{C}}}
$$

Where $\mathrm{m}^{*}$ and $\mathrm{L}$ are the effective mass of electron and the width barrier of $1 \mathrm{D}$ quantum well, respectively. $\mathrm{E}$ and $\mathrm{E}_{\mathrm{C}}$ are the electron energy in and at the bottom of conduction band, respectively.
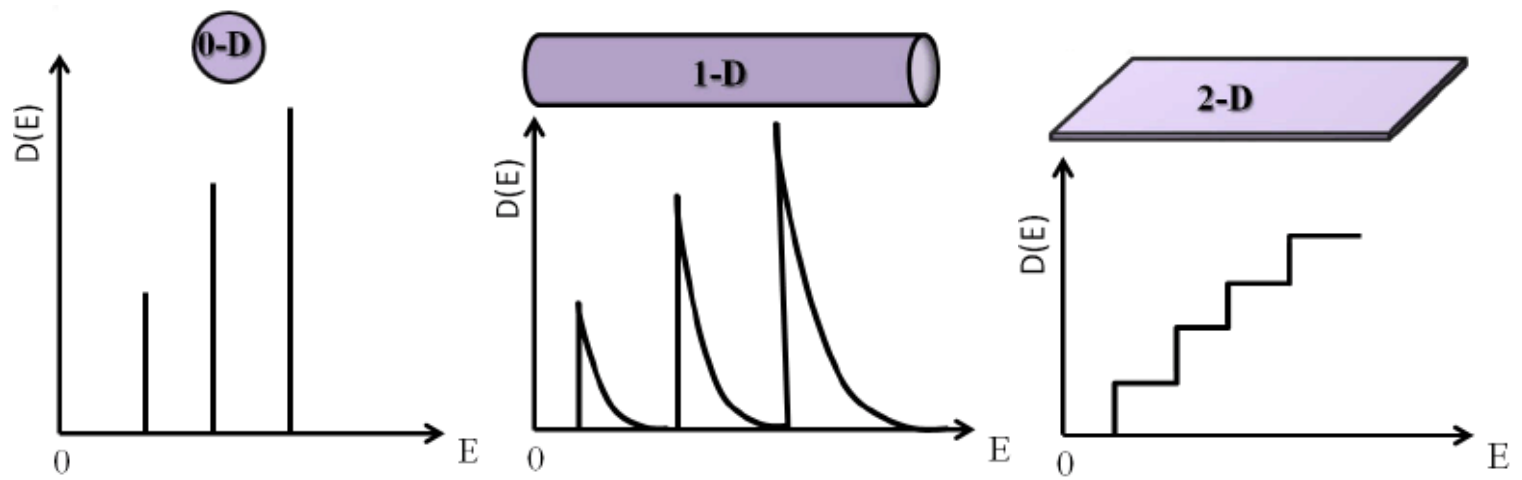

Figure 3: Schematic showing density of state of low dimensional nanostructures [9].

For one dimensional structures the $\mathrm{D}(\mathrm{E})$ has singularities implying high conductivity at these points. The modification of conductivity of sensors based on the 1D system occurs through changes in carrier concentration brought about by electron transport between analyte and the nanostructure. In addition, geometric factors due to 
creation of the depletion layer also affect sensor resistance. Therefore, 1D nanostructures are an ideal candidates for sensing material. This is the primary hypothesis behind the work developed in this thesis. Its validity is tested for $\mathrm{SnO}_{\mathrm{x}}$ nanowires and multiwall carbon nanotubes based sensors for selective and sensitive detection of chlorine and hydrogen gases, respectively.

\subsection{One Dimensional (1D) Nanomaterials for Sensing Applications}

In the last fifteen years, the number of publications involving $1 \mathrm{D}$ nanostructures has exploded due to their unique electronic properties and also due to development of methods for their bulk-scale syntheses. With the lowest dimension for efficient electrical carrier transport, it is ideally utilized for the task of moving charges and photons, i.e., for applications involving nanoelectronics and nanophotonics. They have found wide ranging applications in devices such as transistors, diodes, circuits or as light emitters. In addition, 1D nanostructure, including tubular structures with the unique features such as low mass density, high porosity and extremely large surface area to volume ratios, can provide superior performance as catalysts, fuel cell electrodes, and chemical/biochemical sensors. Out of these, sensor devices based on 1D nanostructures have attracted much greater attention. A comprehensive review of current research activities of chemical sensors on nanotubes, nanorods, nanobelts and nanowires, focusing on experimental principles, design of sensing devices and sensing mechanisms has recently appeared [10]. For example, silicon nanowires based gas sensors have been fabricated for the detection of ammonia and water vapor at room temperature [11]. Sensors utilizing tin oxide nanobelts, operating at $400^{\circ} \mathrm{C}$, can detect carbon monoxide as low as $0.5 \mathrm{ppm}$ [12], . Zinc 
oxide nanorods, grown by plasma-enhanced chemical vapor deposition (PECVD) on polycrystalline $\mathrm{Al}_{2} \mathrm{O}_{3}$ have been used as a chemical sensor for nitrogen dioxide gas detection [13]. Carbon nanotubes have been well-known as not only a chemical sensor for $\mathrm{NO}_{2}$ and $\mathrm{NH}_{3}$ [14], but also a biological sensor [15]. The fundamental sensing mechanism of these gas sensors involves changes in conductivity of nanostructures as result of gas adsorption and interaction between the underlying semiconductor and surface complexes (such as $\mathrm{O}^{-}, \mathrm{H}^{+}, \mathrm{OH}^{-}$), reactive chemical species. The adsorption and charge transport (i.e., change in the $\mathrm{n}$ or $\mathrm{p}$ the carrier concentrations) from the analyte brings the underlying semiconductor in either the depletion or accumulation region leading to changes in channel conductivity.

However, so far, the synthesis and integration for practical, inexpensive sensor devices based on $1 \mathrm{D}$ nanostructures, in term of commercialization, has not received much attention. Integration requires a complete understanding of the device operational parameters, reasonable lifetime and sustained high sensitivity. Therefore, the work presented in this thesis explored ways in which different 1D nanostructures which are easily synthesized and employed for sensing applications for detection of chlorine and hydrogen as an oxidizing and a reducing gases, respectively.

\subsubsection{Chlorine sensor}

Recently, the usage of gas sensors has been on the rise due to their wide array of applications, ranging from safety equipment (explosion, leakage, fire, contamination of poisoning protection) to emission and air quality monitoring, quality assurance, process instrumentation, and measurement technology [16]. In principle, materials that change 
their properties upon exposure to ambient gas can be used as gas sensing materials. Many metal oxides are suitable for gas resistive sensors. These sensors incorporate nanostructures in porous a thick film which provides a high surface to volume ratio enhancing the detection sensitivity. These device operate at elevated temperatures around $300^{\circ} \mathrm{C}[17]$ to enable rapid response time.

In order to detect oxidizing and highly corrosive gases or operate in harsh conditions, appropriate sensing materials must be selected. A prime example of one such gas is chlorine, which is useful in many industrial processes, but is deadly toxic gas at 510 ppm level. A few oxide based $\mathrm{Cl}_{2}$ gas sensor have been developed and summarized in Table 1 below:

Table 1: A brief summary of nanomaterial based chlorine sensors. Adapted and modified from ref [18]

\begin{tabular}{|l|l|l|}
\hline Sensor type & $\begin{array}{l}\text { Detection limit } \\
(\mathrm{ppm})\end{array}$ & $\begin{array}{l}\text { Operation temperature } \\
\left({ }^{\circ} \mathrm{C}\right)\end{array}$ \\
\hline $\mathrm{ZnO}$ & 300 & 400 \\
\hline $\mathrm{WO}_{3} / \mathrm{FeNbO}_{4}$ & 2 & 230 \\
\hline $\mathrm{CdIn}_{2} \mathrm{O}_{4}$ & 0.2 & 250 \\
\hline $\mathrm{SnInO}$ & 3 & 300 \\
\hline $\mathrm{CdSnO}$ & 0.1 & 250 \\
\hline $\mathrm{CuO}-\mathrm{CdIn}_{2} \mathrm{O}_{4}$ & 400 & 250 \\
\hline $\mathrm{In}_{2} \mathrm{O}_{3}$ & 0.5 & 250 \\
\hline $\mathrm{Zn}_{2} \mathrm{In}_{2} \mathrm{O}_{5}-\mathrm{MgIn}_{2} \mathrm{O}_{4}$ & 0.01 & 300 \\
\hline $\mathrm{Mg}_{-} \mathrm{phthalocyanine}$ & 0.18 & 200 \\
\hline $\mathrm{FEP} /$ polyaniline & 100 & 25 \\
\hline $\mathrm{Cu}-$ phthalocyanine & 0.18 & 170 \\
\hline $\mathrm{WO}_{3}$ & 1 & 300 \\
\hline $\mathrm{In}_{2} \mathrm{O}_{3}-\mathrm{Fe}_{2} \mathrm{O}_{3}$ & 0.2 & 250 \\
\hline $\mathrm{NiFe}_{2} \mathrm{O}_{4}$ & 1000 & 250 \\
\hline & &
\end{tabular}

However, the most commonly used in gas sensing materials is nanostructured $\mathrm{SnO}_{2}$ due to its several key advantages over other metal oxides such as low cost of production and bulk-scale availability [19]. Moreover, $\mathrm{SnO}_{2}$ is chemically stable and is 
readily regenerated making it an ideal candidate for detection of such a highly corrosive gas. Additionally, we recently developed an $\mathrm{Sb}$-doped $\mathrm{SnO}_{2} \mathrm{NPs}$ sensor, which operates even at room temperature and can detect $\mathrm{Cl}_{2}$ gas at ppm level. As discussed earlier, in the research presented here replaces the n-doped $\mathrm{SnO}_{2} \mathrm{NPs}$ by n-doped $\mathrm{SnO}_{2} \mathrm{NW}$. The use of NWs, as opposed to NPs, reduces inter-particles electron hopping thereby leading to better sensor performance. This thesis focuses on the study of a highly sensitive, thickfilm $\mathrm{Cl}_{2}$ resistive sensor fabricated from $\mathrm{Sb}$-doped $\mathrm{SnO}_{2} \mathrm{NWs}$. A novel feature of this system is that it also works at room temperature providing good sensor response (50ppb to $10 \mathrm{ppm}$ ) and sensitivity. The work presented here explores the mechanisms of sensor stability and develops chemical models for the sensing mechanism.

\subsubsection{Hydrogen sensor}

Hydrogen is not only one of the fundamental building blocks of living things in nature, but also it is a key starting material in the chemical industry. Recently, hydrogen has emerged as an alternate energy source that can replace dwindling fossil fuel reserves. Additional advantages of its use are that it provides energy security, and it helps reduce global warming. As a colorless, odorless and tasteless flammable gas, hydrogen cannot be detected by human senses, even at concentrations at which it poses an explosion risk. Therefore, a rapid and accurate hydrogen gas sensor with long term stability and wide dynamic range of concentration is desirable.

Numerous approaches are currently being investigated to develop effective hydrogen hydrogen sensors. These sensors employ thermoelectric, electrochemical, optical and acoustic methods of detection [20]. However, the most commercially available hydrogen 
sensors are based on metal oxide materials such as tin oxide. Typically, these types of sensors operate at elevated temperatures to maintain proper performance [21]. Thus, these kinds of sensors still have poor energy efficiency and require complicated fabrication methods $[21,22]$.

Table 2: A brief summary of metal oxide based hydrogen sensors. Adapted and modified from refer [21]

\begin{tabular}{|c|c|c|}
\hline Sensor type & Detection limit (ppm) & Operation temperature $\left({ }^{\circ} \mathrm{C}\right)$ \\
\hline $\mathrm{SnO}_{2}$ nanowires & 10 & 300 \\
\hline $\mathrm{SnO}_{2}$ nanorod & 500 & 230 \\
\hline $\mathrm{SnO}_{2}$ nanobelts & 30,000 & 80 \\
\hline $\mathrm{SnO}_{2}$ film & 10 & 300 \\
\hline $\mathrm{Sn}_{0.9} \mathrm{Ti}_{0.1} \mathrm{O}$ film & 1000 & 450 \\
\hline $\mathrm{Pt}-\mathrm{Ag}-\mathrm{SnO}_{2}$ film & 1000 & 250 \\
\hline Pd-ZnO nanorods & 100 & 25 \\
\hline $\mathrm{WO}_{3}$ film & 1000 & 110 \\
\hline $\mathrm{Pt} / \mathrm{Li}-\mathrm{NiO}$ film & 30,000 & 100 \\
\hline $\mathrm{Pt} / \mathrm{NiO}$ film & 30,000 & 100 \\
\hline $\mathrm{Pt} / \mathrm{Ga}_{2} \mathrm{O}_{3}$ & 10,000 & 610 \\
\hline
\end{tabular}

Recently, palladium has been reported as one of the most promising materials for the detection of $\mathrm{H}_{2}$. Different Pd nanostructures have been proposed as an alternative to the metal oxide to achieve high sensitivity and selectivity toward hydrogen. A thin film of Pd nanoparticle based alloy was employed to detect relative high concentrations of hydrogen at room temperature [23]. The detection principle was based on the formation of Pd hydride, resulting from hydrogen atom incorporation into the Pd lattice structure which changes its overall resistance. These so-called chemiresistor sensors have been further modified to use $\mathrm{Pd}$ nanowire arrays for the specific sensing of $\mathrm{H}_{2}$ [24].

Along with $\mathrm{Pd}$, other one-dimensional nanostructure based sensors of other materials have also attracted a great attention due to their extraordinary properties such as size, unique electronic properties and thus superior sensitivity, and power consumption. One such a sensor employs titanium dioxide nanotubes [25]. Furthermore, one- 
dimensional nanostructures have been incorporated in hybrid structure sensors. For example, Pd-functionalized tin oxide nanowires exhibited a dramatic improvement in sensitivity toward hydrogen [26]..

It is well-known that carbon nanotubes (CNTs) have become one of the most exciting one-dimensional nanomaterials due to their extraordinary electrical properties that have been exploited in a wide variety of applications. One of these is the sensing application mentioned above. However, very weak adsorption of hydrogen gas results in poor electronic interaction with CNT and even with defective CNTs, poor sensitivity was observed [27]. Therefore, CNTs composites were proposed as a sensing material for hydrogen detection and demonstrated through many studies. Enhanced $\mathrm{H}_{2}$ sensitivity was observed when a composite based on Pd evaporated on single wall carbon nanotubes was used as hydrogen sensing material $[27,28]$. Pd nanoparticles were attached to single walled CNTs for a better sensor performance [29]. Even though there was some preliminary explanation about the electronic interaction between adsorbates $\left(\mathrm{H}_{2}\right)$, catalysts (Pd) and sensing materials (CNTs) in this type of structure, the understanding about this sensing mechanism is still not fully established. Especially, in the case of replacing semiconducting SWNTs by multiwall CNTs, not much work has been conducted.

In this study, a better hydrogen gas sensor performance operating at room temperature and using hybrid structure of MWCNTs and Pd nanoparticles is reported. The work presented here provides a new green synthetic method developed to produce defective MWCNTs in a simple evacuated glass tube. These CNTs, decorated with Pd 
NPs, show remarkably wide dynamic range for $\mathrm{H}_{2}$ detection from a few ppm to $4 \%$. The sensing mechanism during chemiresistive process is also established that entails a carrier inversion in the p-type nanotubes and even observation of negative differential resistance. These phenomena can be explained in terms simple reduction of CNTs by atomic/anionic hydrogen generation catalyzed by Pd NPs. 
CHAPTER 2. INSTRUMENTATION, DEVICE CHARACTERIZATION AND NANOSTRUCTURE SYNTHESIS

\subsection{Synthesis and Sensor Fabrication}

The synthesis of nanostructures includes bulk scale preparation of $\mathrm{SnO}_{2} \mathrm{NWs}$, CNTs, and their doped counterparts. Sensor fabrications based on these nanomaterials for of $\mathrm{H}_{2}$ and $\mathrm{Cl}_{2}$ detection are also presented in this section.

\subsubsection{Sample preparation for $\mathrm{SnO}_{2} \mathrm{NWs}$}

2.1.1.1 Synthesis of undoped and doped $\mathrm{SnO}_{2} \mathrm{NWs:}$

\section{A. Undoped $\mathrm{SnO}_{2} \mathrm{NWs}$}

Method I: The precursors Sn NPs were synthesized by reducing a thoroughly mixed solution of $50 \mathrm{ml}$ of $0.05 \mathrm{M} \mathrm{SnCl}_{4} \cdot 5 \mathrm{H}_{2} \mathrm{O}$ and $0.5 \mathrm{~g}$ of 1, 10-phenanthroline with $100 \mathrm{ml}$ of $0.1 \mathrm{M} \mathrm{NaBH}_{4}$ aqueous solution. The reducing solution was introduced drop wise to synthesize phenanthroline capped Sn nanoparticles [30]. NPs were separated from the reaction mixture by centrifuging $(10,000 \mathrm{rpm}$ for 15 minutes) after two hours of reaction time. The precipitate was dried at $50^{\circ} \mathrm{C}$ in $2 \mathrm{hrs}$ on a hotplate. $0.2 \mathrm{~g}$ of 1,10 phenanthroline capped Sn NP powder was mixed with a mixture of $0.4 \mathrm{~g}$ of $\mathrm{NaCl}$ and 0.6 $\mathrm{g}$ of $\mathrm{KCl}$ (eutecting point at $657 \mathrm{C}^{\circ}$ ), grounded into a fine powder, and heated at $750{ }^{\circ} \mathrm{C}$ for $2 \mathrm{~h}$ in a furnace in air. The molten mixture was then slowly cooled to room temperature. The solidified product was washed several times with deionized (DI) water, to remove $\mathrm{KCl}$ and $\mathrm{NaCl}$. Their removal was tested with $0.05 \mathrm{mM} \mathrm{AgNO}_{3}$ solution for any residual $\mathrm{Cl}^{-}$anion. The wet powder was dried overnight in an oven kept at $100^{\circ} \mathrm{C}$ to remove water. 
Method II: A modified synthetic method could be adapted to produce much higher quantity products of $\mathrm{SnO}_{2} \mathrm{NWs} .5 \mathrm{~g}$ of $\mathrm{SnCl}_{4} .5 \mathrm{H}_{2} \mathrm{O}$ was dissolved in $150 \mathrm{mls}$ of water. The solution was stirred for 15 minutes before drop wise addition of $50 \mathrm{ml}$ of $2 \mathrm{~g}$ $\mathrm{NaBH}_{4}$ dissolved in water. The mixture turned black, indicating formation of the oxide coated Sn NPs. The precipitate was collected by filtering and left to dry in air overnight. $2 \mathrm{~g}$ of the above precursor was mixed with $0.2 \mathrm{~g}$ of 1,10-phenanthroline, $4 \mathrm{~g}$ of $\mathrm{NaCl}$ and 6 $\mathrm{g}$ of $\mathrm{KCl}$, ground into a fine powder, and kept at $750^{\circ} \mathrm{C}$ for $2 \mathrm{hr}$ in an oven in air.

B. n-doped $\mathrm{SnO}_{2} \mathrm{NWs}$

For n-doping, varying amount of antimony chloride (1-5\% at. wt. in relation to Sn) was added to the initial solution of $\mathrm{SnCl}_{4} \cdot 5 \mathrm{H}_{2} \mathrm{O}$. The rest of the synthetic procedure was identical to the synthesis of undoped NWs as described in method II. Note that the actual amount of dopant inserted in NWs could be different than the concentration of dopant atoms used during synthesis.

\section{Li-dopedSnO 2 NWs}

Method III: For Li-doping, LiCl replaced antimony chloride as a dopant. The amounts of lithium chloride added to the initial reaction mixture ranged from 1 to $5 \mathrm{wt} \%$. The precursors, Sn NPs coated with a thin layer of tin oxide, were synthesized without capping agents by adding $0.35 \mathrm{~g} \mathrm{SnCl}_{4} \cdot 5 \mathrm{H}_{2} \mathrm{O}$ with $0.1 \mathrm{M} \mathrm{NaBH}_{4}$ aqueous solution containing $\mathrm{LiCl}$. The mixture turned black, indicating formation of oxide coated Sn NPs. The precipitate was collected by filtering and left to dry in air over night. $0.2 \mathrm{~g}$ of the above precursor was mixed with $0.2 \mathrm{~g}$ of 1,10 -phenanthroline, $0.31 \mathrm{~g}$ of $\mathrm{LiCl}$ and $0.6 \mathrm{~g}$ of $\mathrm{KCl}$ (eutectic point at $353 \mathrm{C}^{\circ}$ ), ground into a fine powder, and kept at $480 \mathrm{C}^{\circ}$ for $2 \mathrm{hr}$. 
The molten mixture was then slowly cooled to room temperature at a cooling rate of $4^{0} \mathrm{C} /$ minute. The products were collected and purified as in the case of undoped NWs by multiple washing with DI water till $\mathrm{Cl}^{-}$ions were not detected by the $\mathrm{AgNO}_{3}$ test.

\subsubsection{Sensor fabrication using n-doped $\mathrm{SnO}_{2} \mathrm{NWs:}$}

To fabricate the sensor, a simple powder compression technique was employed. For the chlorine sensor, $2 \mathrm{~g}$ of $\mathrm{Sb}$-doped $\mathrm{SnO}_{2}$ were mixed with $0.5 \mathrm{ml}$ of $2 \%$ ethyl silicate in acidified ethanol. The paste was compressed using a pressure of $5463 \mathrm{~kg} \mathrm{~cm}^{-2}$ to yield a $13 \mathrm{~mm}$ pellet with a thickness of $800 \mu \mathrm{m}$. The pellet was heated at $450{ }^{\circ} \mathrm{C}$ for 30 minutes. It was then mounted onto a glass substrate and copper contacts attached using conductive silver epoxy.
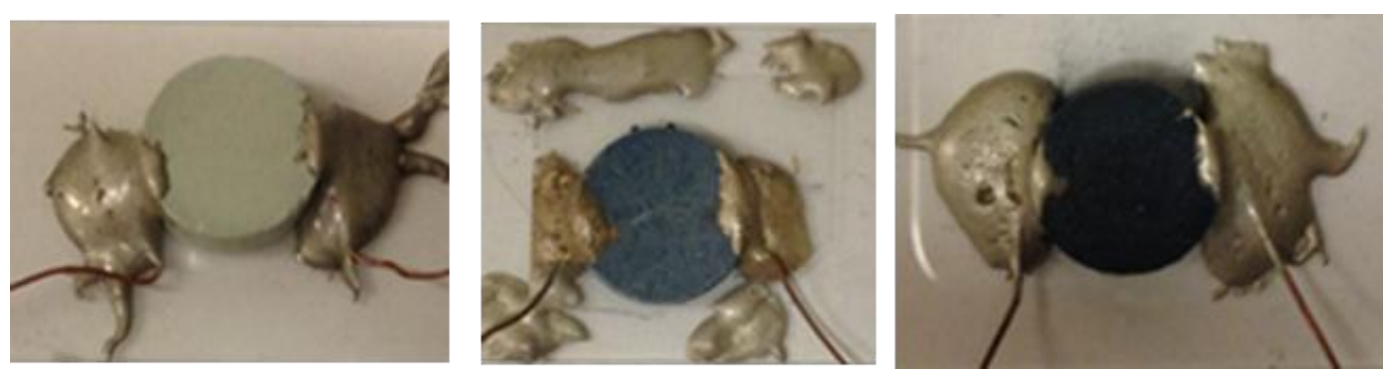

Figure 4: Photographs of $1 \%, 5 \%$ and $10 \% \mathrm{Sb}$-doped $\mathrm{SnO}_{2} \mathrm{NWs}$ based sensors (from left to right). Note the increase in the dopant concentration leads to increasingly blueish color to the pellets. Electrical contacts (silver epoxy and copper wire) and compressed nanoparticle pellet are mounted on a microscope glass slide.

\subsubsection{Sample preparation for CNTs}

\subsubsection{Synthesis of undoped MWCNTs:}

A. Dual chamber method $(\operatorname{method} A)$ :

This method was carried out through two steps including the synthesis of Ni nanoparticles as catalyst precursors, which are used as a catalyst for the synthesis of CNTs through vapor-liquid-solid (VLS) mechanism. 
Synthesis of Ni nanoparticles: A $5 \mathrm{~nm}$ thin film of Nickel on silicon substrate was deposited by using the physical vapor deposition (PVD) system (see below). The thin film covered silicon substrate was then placed in a glass tube, which was evacuated to 80mTorr pressure. After evacuation, the glass tube was sealed off. The nickel nanoparticle catalysts were formed by annealing at $675^{\circ} \mathrm{C}$ for 15 minutes which breaks the Ni thin film into nano-size islands by dewetting.

Synthesis of undoped CNTs: $10 \mathrm{mg}$ of Naphthalene was first placed into the bottom of a borosilicate glass tube $(0.8 \mathrm{~cm}$ O.D. X $0.6 \mathrm{~cm}$ I.D.), ensuring that the solid did not adhere to the sidewalls. The tube was sectioned into two chambers by thinning its diameter to a neck with a propane torch at approximately $5 \mathrm{~cm}$ away from the bottom of the tube. The catalytic Ni nanoparticle coated substrate was then inserted into the top half of the tube. The thinned neck prevents the substrate from falling into the precursor, naphthalene. The top of the tube was thinned to a neck with a propane torch approximately $25 \mathrm{~cm}$ from the bottom of the tube for ease of sealing. The whole tube was connected to glass vacuum line at pressure of 80 mTorr. After evacuation, the glass tube was sealed off resulting in a $20 \mathrm{~cm}$ duo-chamber glass reaction vessel as shown in Figure 5 below

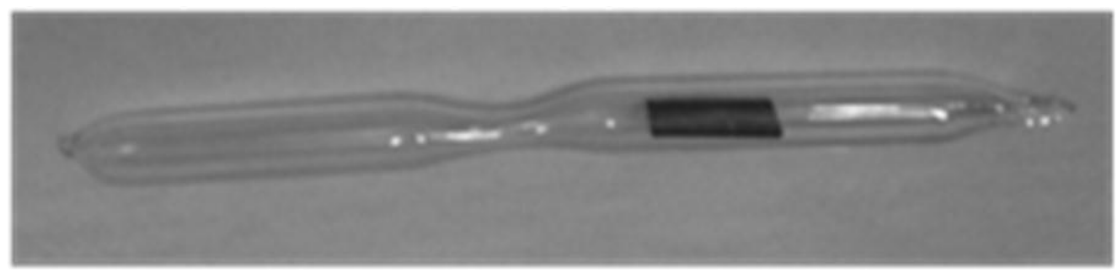

Figure 5: An optical image of duo-chamber glass reaction vessel for the synthesis of MWCNTs. Catalyst is deposited on the silicon substrate in the right side chamber; and Naphthalene, the CNT feed stock, is kept in the left chamber. 
The two chambers were heated to different temperatures using separate heating tapes. To ensure that the $\mathrm{Si}$ substrate reaches the desired temperature, the substrate chamber temperatures was adjusted first. The naphthalene containing glass chamber temperature was adjusted once the substrate temperature reached the desired range. . The naphthalene containing chamber temperature was kept at $100^{\circ} \mathrm{C}$ at the beginning of the reaction. Over the course of reaction (about 2 hours) the chamber temperature rose to about $200^{\circ} \mathrm{C}$ since the substrate side is kept at about $600^{\circ} \mathrm{C}$. This is termed controlled synthesis and illustrated in Figure 6a.

B. Single Chamber method (method B):

In this case the procedure was carried out similarly to method A, but the length of glass tube was changed to $10 \mathrm{~cm}$ after sealing. Both Ni nanoparticles coated substrate and naphthalene were located in single chamber. The whole tube was then inserted at once into the furnace kept at desired temperature. This is termed as uncontrolled synthesis. See Figure 6b. 

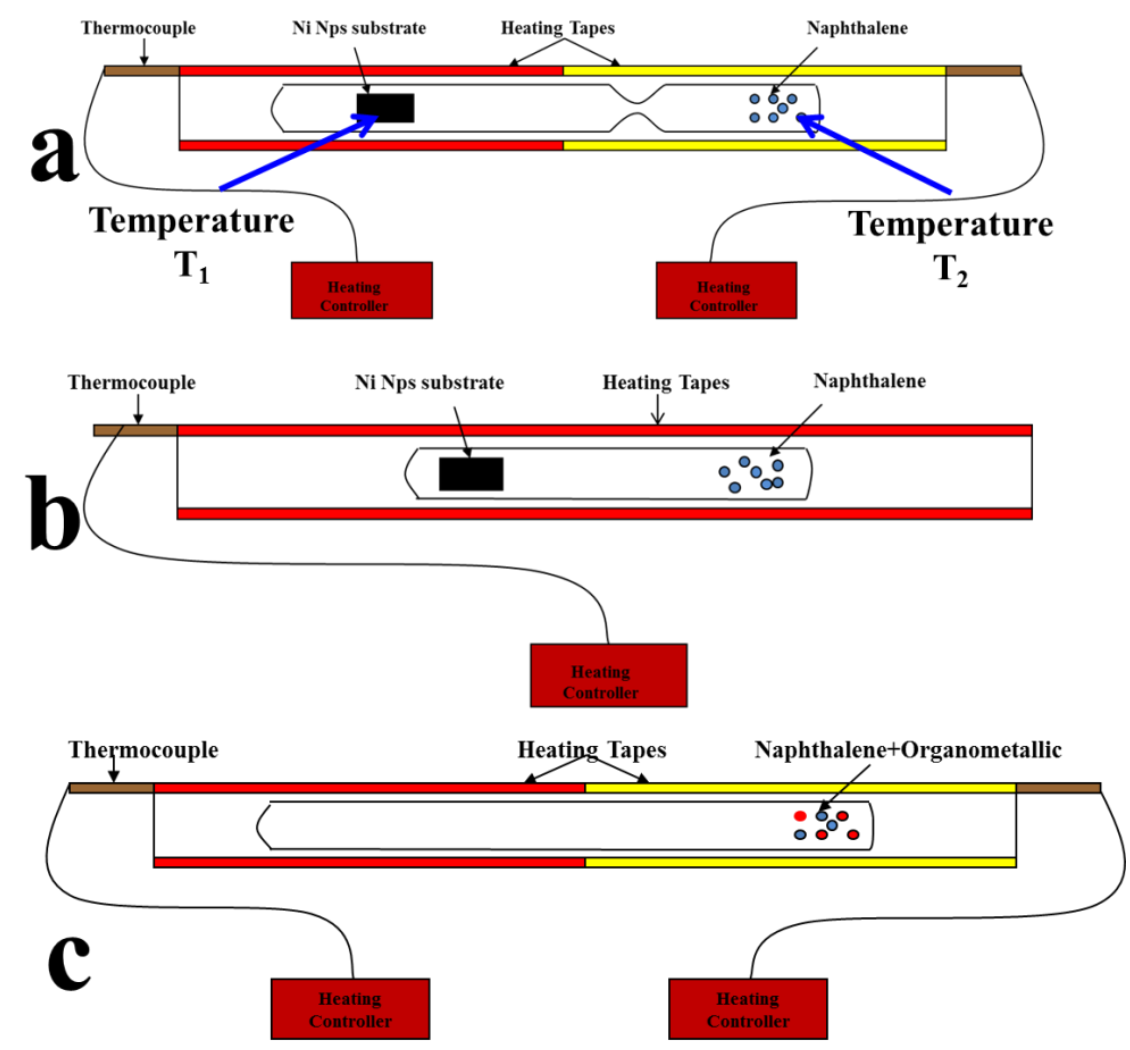

Figure 6: Schematic illustration of CNTs synthesis from three different synthetic methods. a) Dual chamber separating catalyst from the reactant. Independent temperature control on the two chambers was obtained using 2 heating sources (heating tapes, Omega, STH051-080). b) Single chamber with one heating source. c) Single chamber reactor where catalyst and reactant are mixed.

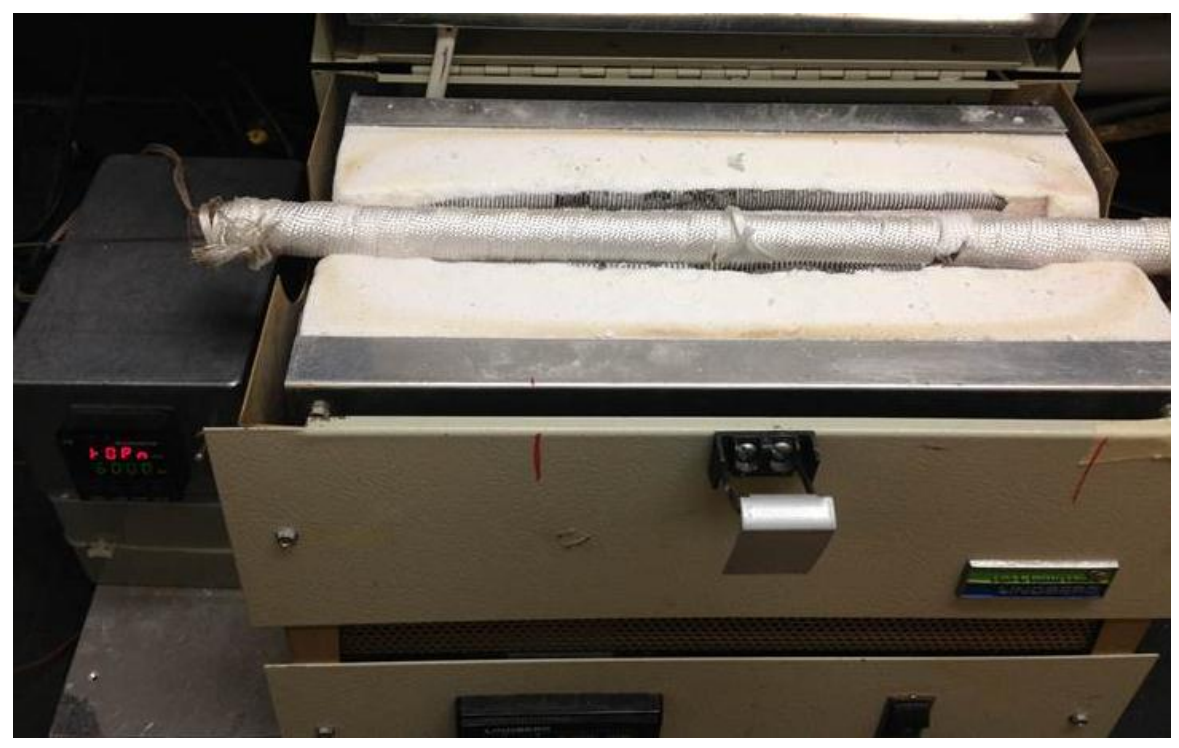

Figure 7: The photograph of the custom-built CNT synthesis system housed inside a tube furnace. 


\section{Using Ferrocene and Cobaltocene as catalyst generators (method C):}

The preparation method was similar to the previous methods using vacuum sealed glass tubes. However, no substrate coated with catalysts was utilized. CNTs were grown directly on the inner wall of the glass tube (Figure 6c). A mixture of $15 \mathrm{mg}$ of naphthalene mixed thoroughly with ferrocene or cobaltocene with mass ratio 5:1 was placed in one side of the tube and vacuumed down to $60 \mathrm{mTorr}$ followed by sealing. The temperature of this side was ramped up from $100^{\circ} \mathrm{C}$ to $200^{\circ} \mathrm{C}$ with the heating rate of $1^{\circ} \mathrm{C} /$ minute while the other side was kept at $625^{\circ} \mathrm{C}$.

\subsubsection{Synthesis of doped MWCNTs:}

In this work, the "green approach" was demonstrated by not using toxic gases as precursors or dopants. Dopants were based on the use of boron and nitrogen containing organic compounds, which are in the solid phase at room temperature. Triphenylborane and benzylamine (Figure 8) are considered as p- and n- type substitutional dopants for carbon, respectively.<smiles>c1ccc(B(c2ccccc2)c2ccccc2)cc1</smiles>

Triphenylborane

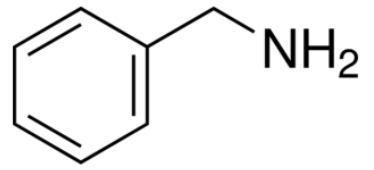

Benzylamine

Figure 8: Molecular structure of doping agents for CNTs

Boron- and Nitrogen-doped CNTs were synthesized using various levels of triphenylborane and benzylamine as p- and n-type doping sources, respectively. The 
syntheses were carried out using a non-substrate pathway (method C). Different amounts of dopant were added to the mixture of cobaltocene and naphthalene to alter the doping concentrations.

\subsubsection{Synthesis of palladium nanoparticles attached MWCNTs}

The attachment of palladium nanoparticles on the walls of CNTs was adapted from Karousis's method [31]. $5 \mathrm{mg}$ of commercial MWCNTs were suspended in $10 \mathrm{mls}$ of $0.05 \mathrm{M}$ aqueous sodium dodecyl sulfate (SDS) solution along with the $10 \mathrm{mg}$ of palladium acetate. This mixture was ultrasonicated for 20 minutes and refluxed at $90^{\circ} \mathrm{C}$ for $6 \mathrm{hrs}$. The palladium nanoparticle decorated CNTs were collected and separated from the reaction mixtures by centrifugation and repeated washings with ethanol to remove the excess adsorbed surfactant. The precipitate was then suspended in dimethylformamide solvent (DMF).

The sensor was constructed by depositing a thin film of the above suspension. One $\mathrm{ml}$ of the final solution was slowly deposited drop wise and evaporated at $40^{\circ} \mathrm{C}$ on a $5 \mathrm{~mm} \times 10 \mathrm{~mm}$ silicon substrate. Conductive silver epoxy was used as the material for electrodes.

\subsubsection{Sensor construction from as-synthesized MWCNTs and Palladium nanoparticles}

The sensor construction for as synthesized-undoped CNTs by the sealed glass tube method was also investigated for hydrogen detection. In this case, the CNT product synthesized from method C was employed. A $3 \mathrm{~cm}$ glass tube which has CNTs deposited was cut and gently placed into $10 \mathrm{ml}$ of $0.05 \mathrm{M}$ aqueous sodium dodecyl sulfate (SDS) solution containing $10 \mathrm{mg}$ of palladium acetate. Attachment of Pd nanoparticles onto 
CNTs was achieved similarly through the above synthetic process, but without the sonication. This portion of glass tube after synthesis was thoroughly washed many times with water and completely dried in the oven at $100^{\circ} \mathrm{C}$ in $2 \mathrm{hrs}$. Conductive silver epoxy was deposited on both edges of the tube and used as electrodes for sensor measurements.

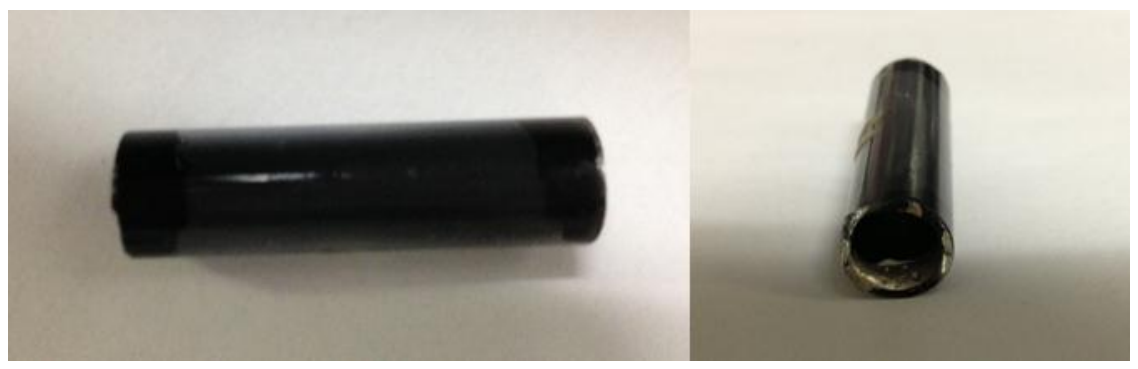

Figure 9: A photograph of fabricated CNT based sensor using method C. Electrical contact to the two sides was provided by using silver epoxy and copper wire as shown in Figure 4.

\subsection{Electron Microscopy}

Scanning electron microscopy (SEM)

An FEI Siron XL30 model SEM was used for electron microscopy imaging. The imaging studies were performed at an accelerating voltage of $5 \mathrm{kV}$, and beam current of $\sim 300 \mu \mathrm{A}$.

Transmission electron microscopy

The TEM microscope employed in this study was a FEI Technai F-20 TEM operating at an accelerating voltage of $200 \mathrm{kV}$. A column vacuum of less than $0.10 \mu$ Torr was routinely achieved. TEM studies included bright field, dark field, selected area diffraction, EDX analysis and high resolution lattice plane imaging.

\subsection{Physical Vapor Deposition (PVD)}


Physical Vapor Deposition (PVD) system was made by Lesker Thin Film Deposition System Model KJLC AXXIS. Various film thicknesses from 5 to $20 \mathrm{~nm}$ of Ni catalyst on Si substrate were deposited to optimize the growth conditions for CNTs.

\subsection{Infrared Spectroscopy (FTIR)}

Fourier transform infra-red (FTIR) spectra were acquired on a Perkin-Elmer RXI instrument. Desorbed gases from the sensor chambers were captured in a gas sampling cell having transparent salt plate windows for Infra-red spectroscopic analysis.

\subsection{UV-Visible Spectroscopy}

Optical absorption properties NWs were investigated by UV-Vis light using a Perkin Elmer spectrometer model UV-3600 UV-Vis-NIR. The spectra were collected in the range from $250 \mathrm{~nm}$ to $900 \mathrm{~nm}$ wavelength. The analysis of absorption yielded the band-gap of as-synthesized $\mathrm{SnO}_{2} \mathrm{NWs}$.

\subsection{Photoluminescence Spectroscopy}

In this dissertation work, the optical photoluminescence studies were performed on a Shimadzu spectrofluorophotometer RF-5301PC, using a xenon light source with 3 $\mathrm{nm}$ bandwidth. The detector was placed on an axis at $90^{\circ}$ angle from that of the incident beam. The sample holder was a rectangular quartz cell with all 4 polished vertical slides. This holder was adjusted at right angle of the incident beam and detector to permit the transmitted radiation to pass through the test solution without contaminating the output signal received by the detector. A sample with $\mathrm{SnO}_{2} \mathrm{NWs}$ dispersed in deionized water was first scanned at different excitation wavelengths, then at a fixed emission wavelength, to determine the optimal excitation wavelength. 


\subsection{Raman Spectroscopy}

Raman spectroscopy is a spectroscopic technique based on the shifts in wavelength of the inelastically scattered radiation. In principle, when a monochromatic laser beam is incident on a material of choice, photons of the laser are absorbed. Majority of the light is Rayleigh/elastically scattered, wherein the energy of emitted photons is equal to incident photons from the laser. However, some photons interact inelastically with the material and undergo a change in energy. Frequency, v, of the reemitted photons are shifted down (Stokes scattering) or shifted up (anti-Stokes scattering) in comparison to incident photon frequency. These shifts are called "Raman Shifts" and provide a wealth of information about phonon/vibrational energies [32]. A simplified energy diagram illustrating these concepts is shown on the Figure 10.

The primary purpose of conducting Raman spectroscopic studies was to get an indirect indication of sample doping. Both $\mathrm{CNT}$ and $\mathrm{SnO}_{2}$ have well characterized Raman spectra which have been used in the past to interpret the extent of doping by considering the shift in the spectral peak positions as a function of dopant concentration. This was the only method available to characterize the extent of doping on a molecular scale in the synthesized samples. 


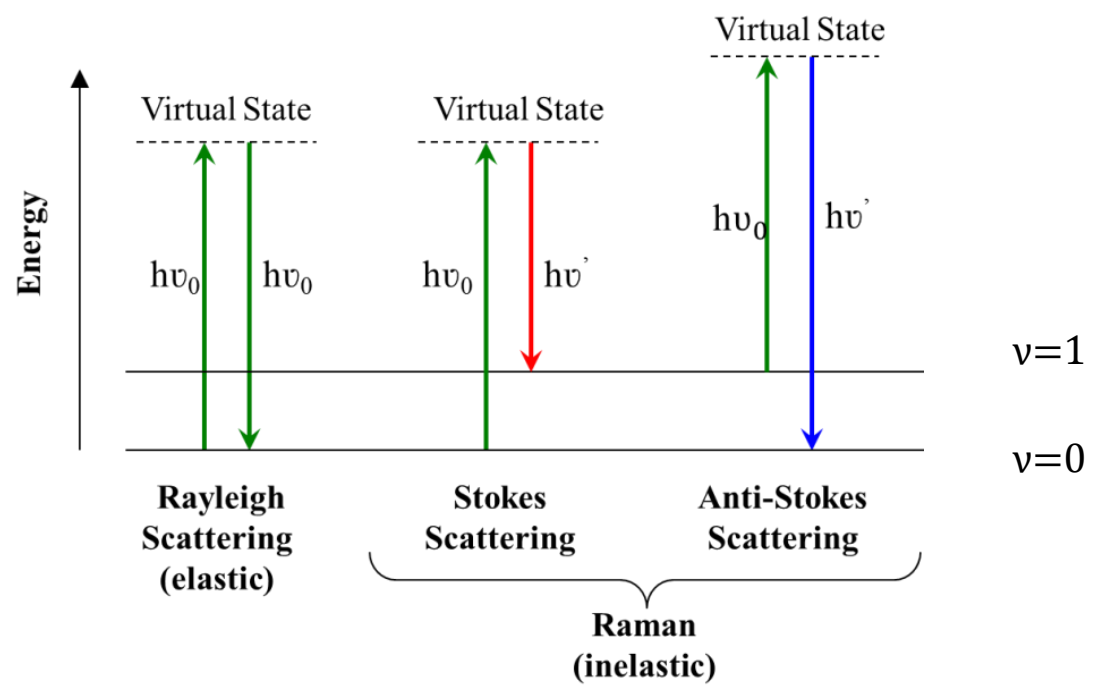

Figure 10: Raman transitional scheme: different types of scattering are achieved depending on energy of emitted photon (hv') relative to the energy of absorbed photon $\left(h v_{0}\right)$. The bottom two vibrational energy levels as $v=0$ (ground state) and first excited vibrational state $v=2$ are shown.

Raman spectroscopic measurements were carried out using a home-built system illustrated in Figure 11. It consisted of four majors components including excitation source (laser), samples illuminating system and light collection optics, wavelength selector (spectrometer) and detector charge-couple device (CCD). Light from an Argon ion laser at $532 \mathrm{~nm}$ wavelength passed through a laser line filter making the beam highly monochromatic. The beam was then reflected through a couple of beam scattering systems (BS) and focused onto the sample through an objective lens. The Rayleigh and Raman scattered light was collected through the same objective lens and passed through a notch filter to selectively block the Rayleigh scattering. The remaining Raman scattered light passed through a spectrometer (diffraction grating) and collected by a detector CCD to produce Raman spectra on a computer after appropriate signal averaging.

Since a multichannel dispersive spectrometer was used, Raman spectra were collected as peak positions in pixel unit versus peak intensity. The data was analyzed 
after converting the unit of pixel to unit of energy $\left(\mathrm{cm}^{-1}\right)$. Futher refinement in the precise peak position was achived through curve fitting, which is demonstarted in the following sections. In addition, the Si peak was used as a reference to calibrate the system. It has been reported that the first-order Raman spectrum of silicon yields a strong signal at $\sim 520$ $\mathrm{cm}^{-1}$ [33]. Therefore, the offset of peak positions on Raman spectra were corrected based on the position of the Si Raman peak, from the silicon substrate bearing $\mathrm{CNT}$ or $\mathrm{SnO}_{2}$ samples.

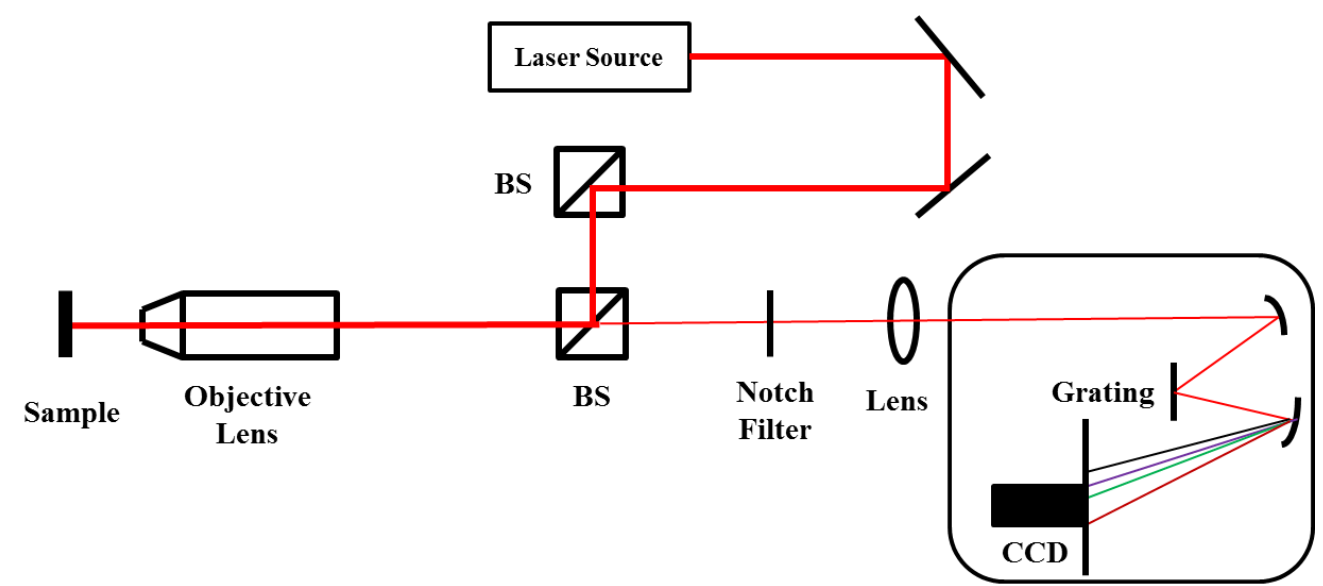

Figure 11: Schematic showing the home-built Raman system with major components including excitation source (Lighthouse Photonic, model Sprout-G), optic system (Thorlabs, model VM1), wavelength selector (Spectra Pro, model 2150i) and detector (Princeton Instrument, model 7527-0001).

\subsection{Sensor testing system}

The sensor performance was tested in a home-built test chamber at room temperature [34]. A schematic view of the chamber made from glass is shown in Figure 12. A two wire resistance of the sensor material was monitored by a Fluke 8840A multimeter which was interfaced to a computer on a National Instrument's GPIB-PCII interface. In this method, a continuous flow of diluted test gas was employed as opposed to fixed dosage of analyte gas to the chamber. This was done to prevent the problem of 
gas adsorption on the chamber wall which reduces the concentration analyte. The sensor response time was measured by analyzing resistance versus time data. To measure recovery time, a flow of fresh air was maintained in the chamber and the sensor temperature was adjusted. The sample temperature can be adjusted between $25^{\circ} \mathrm{C}$ and $120^{\circ} \mathrm{C}$ by varying the resistive heater voltage (0-5volts). A Cr-Al thermocouple was mounted on the heater to measure the sensor operating temperature. The output of the thermocouple was fed to a temperature indicator Mastech (MAS-345) multimeter.

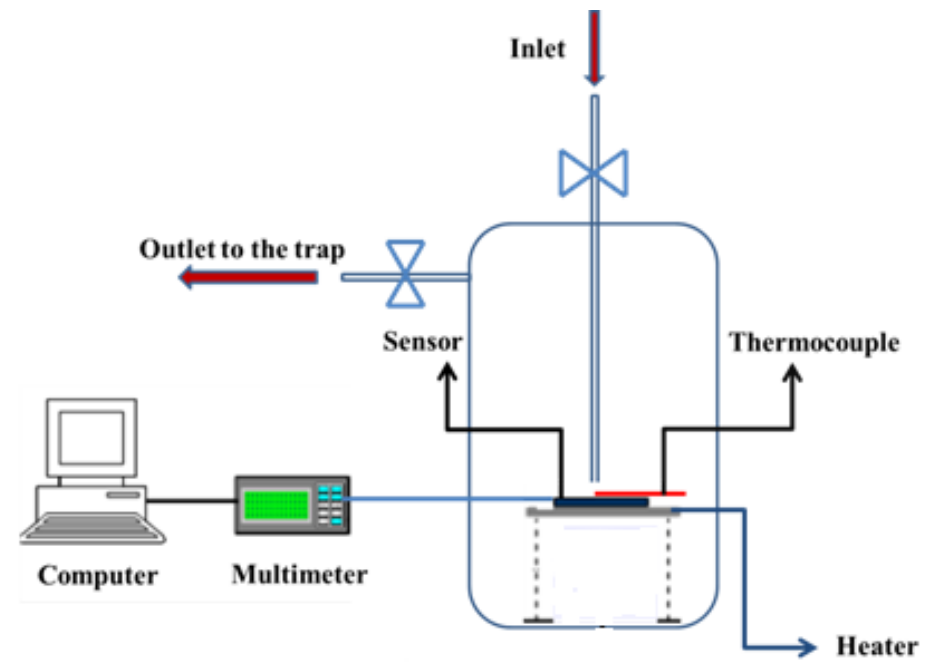

Figure 12: A schematic view of the gas test chamber

The exhaust gas was bubbled through alkali solution to trap the toxic $\mathrm{Cl}_{2}$ gas. For the hydrogen source, forming gas $\left(4 \% \mathrm{H}_{2}\right.$ in $\left.96 \% \mathrm{~N}_{2}\right)$ was used as a primary source of hydrogen. Diluted $\mathrm{H}_{2}$ gas was directly released in the chemical hood. The test gas diluting system, illustrated in Figure 13 (right), was built on analyte and diluting gas sources as well as flow regulators (Dwyer A25431, Aalborg PMR1011029). 


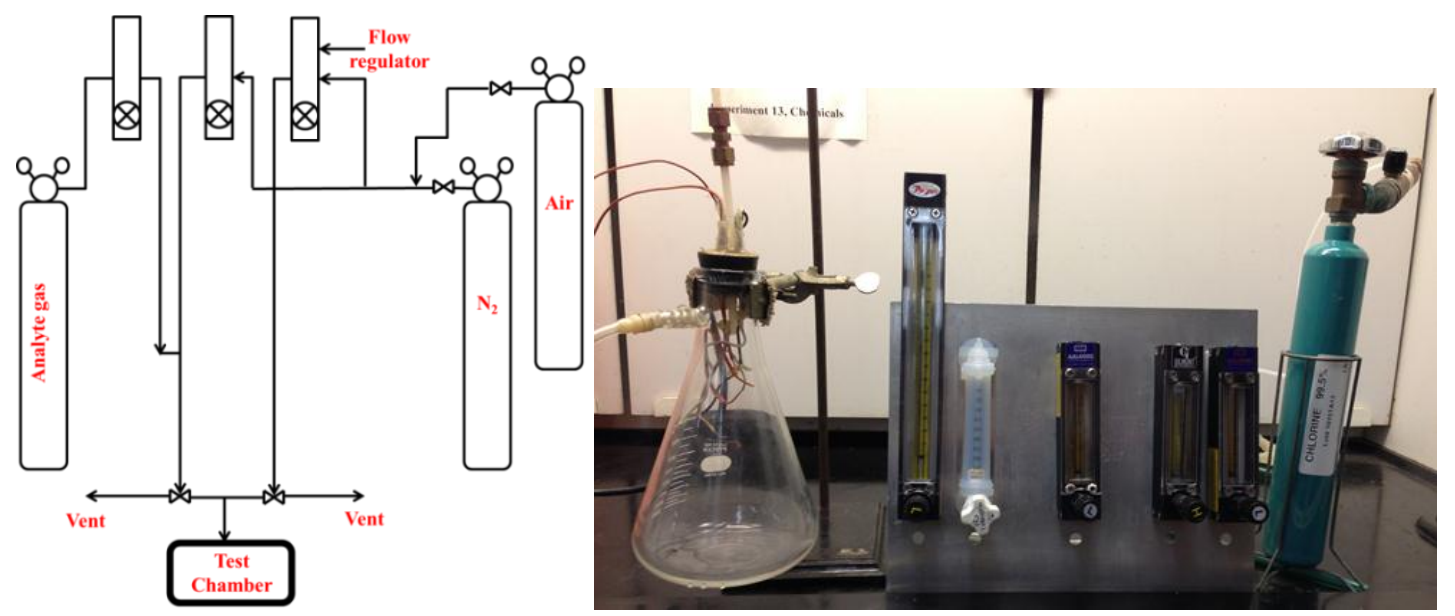

Figure 13: Schematic of gas diluting system (left) and the photograph of home-built sensor testing system (right). 


\section{CHAPTER 3. TIN OXIDE NANOWIRES $\left(\mathrm{SnO}_{2} \mathrm{NWs}\right)$}

It is well-known that metal oxides have a wide range of conductivity ranging from wide band gap insulators to metal conductors and superconductors. Amongst metal oxide materials, tin oxide $\mathrm{SnO}_{2}$ is commonly used in electronic applications. A key benefit of this semiconductor over the contemporary semiconductors such as silicon or gallium arsenide is that it is chemically corrosion resistant. Thus, this wide band-gap semiconductor $(3.56 \mathrm{eV})$ has found uses in electronics such as transparent conductors [3538] for flat panel displays, solar cells, and in chemistry as heterogeneous catalysts [3944] and solid state gas sensing materials $\left(\mathrm{O}_{2}, \mathrm{CO}, \mathrm{Cl}_{2}, \mathrm{NO}_{2}, \mathrm{NH}_{3}\right.$ etc. $)$ [45-50]. Sensors of $\mathrm{SnO}_{2}$ nanowire provide a useful comparison with the CNT nanotube based sensors as discussed below. In this chapter, background and significance of $\mathrm{SnO}_{2}$ nanomaterials are reviewed. Following these, studies of synthesis, characterization and sensor performance of n-doped $\mathrm{SnO}_{2} \mathrm{NWs}$ for chlorine gas detection are presented.

\subsection{Background and Significance}

The review herein describes physical properties of $\mathrm{SnO}_{2}$ nanomaterial including crystal structure, stoichiometry and defect structure as well as Raman characteristics of $\mathrm{SnO}_{2}$ rutile structure.

\subsubsection{Crystal Structure}

$\mathrm{SnO}_{2}$ crystallizes in a tetragonal rutile structure with space group $\mathrm{D}_{4 \mathrm{~h}}{ }^{14}[29,30]$. The unit cell contains six atoms, two tin and four oxygen atoms, as illustrated in Figure 14. Each tin atom is at the center of six oxygen atoms placed approximately at the corners of a regular octahedron, and every oxygen atom is surrounded by three tin atoms 
approximately at the corners of an equilateral triangle. Thus, it is a structure of $6: 3$ coordination. The lattice parameters determined by Baur are $\mathrm{a}=\mathrm{b}=4.737 \AA$ and $\mathrm{c}=$ $3.185 \AA[16,51]$. The ionic radii for $\mathrm{O}^{2-}$ and $\mathrm{Sn}^{4+}$ are 1.40 and $0.71 \AA$, respectively. Metal atoms (cations) are located at positions $(0,0,0),(0,1,0),(1,0,0),(0,0,1),(1,1,0),(1,0,1)$, $(0,1,1),(1,1,1)$ and $(1 / 2,1 / 2,1 / 2)$ in the unit cell, and the oxygen atoms (anions) at $\pm(\mathrm{u}, \mathrm{u}, 0)$, $(\mathrm{u}, \mathrm{u}, 1)$, and $\pm(1 / 2+\mathrm{u}, 1 / 2-\mathrm{u}, 1 / 2)$, where the internal parameter, $\mathrm{u}$, takes the value 0.307 . Each cation has two anions at a distance $\left(\mathrm{d}_{1}\right)$ of $(2 \mathrm{ua})^{1 / 2}(2.053 \AA)$ and four anions at distance $\left(d_{2}\right)\left[2(1 / 2-u)^{2}+\left({ }^{c} / 2 a\right)^{2} a\right]^{1 / 2}(2.597 \AA)$. Each anion is seen to be bonded to cations in a planar trigonal configuration.

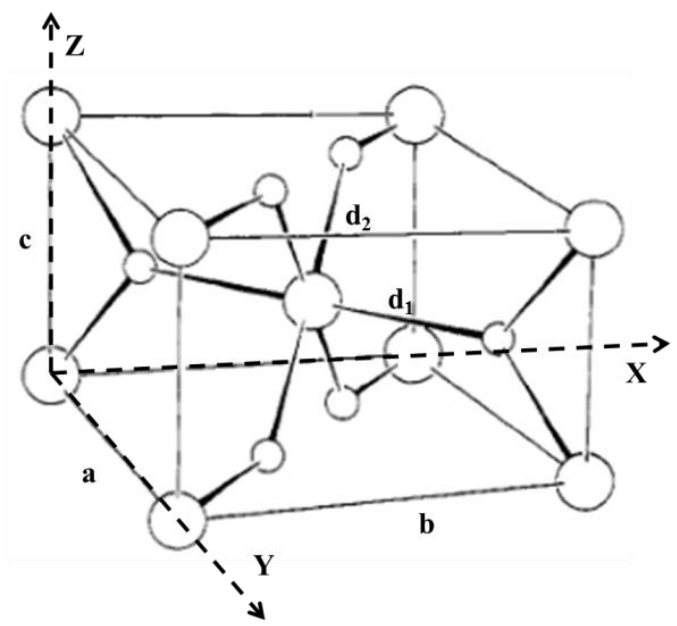

Figure 14: Unit cell of the crystal structure of $\mathrm{SnO}_{2}$ : large circles indicate tin atoms and the small circles indicate oxygen atoms.

\subsubsection{Stoichiometry, defect structure and conductivity}

It is well-known that physical properties of oxides strongly depend on the deviation from the stoichiometric composition $\left(\mathrm{SnO}_{2-\mathrm{x}}\right.$ as opposed to $\mathrm{SnO}_{2}$ native disorder) and on the nature and concentrations of foreign atoms incorporated into the 
crystal lattice [52]. It is generally agreed that $\mathrm{SnO}_{2}$ in its undoped form is an n-type, wide-band gap semiconductor with a bandwidth of $\mathrm{E}_{\mathrm{g}}=3.6 \sim 4.0 \mathrm{eV}$

Therefore, creation of oxygen vacancies $\left(V_{\ddot{O}}\right)$ as a result of $\mathrm{O}_{2}$ leads to electron donation to the conduction band [53]

$$
\mathrm{SnO}_{2} \leftrightharpoons \mathrm{Sn}_{\mathrm{Sn}}+\mathrm{O}_{\mathrm{O}}+\mathrm{V}_{\ddot{O}}+\frac{1}{2} \mathrm{O}_{2}(g)+2 e^{\prime}
$$

Here Kröger-Vink notation [54] is used, wherein superscripted dots and primes indicate positive and negative charges respectively. The doubly ionized (passively charged) oxygen vacancies $\left(V_{\ddot{o}}\right)$ serve as main the intrinsic defects and determine the electric properties of the material, and excess electrons (eq. 3.1) donated to the conduction band making the tin oxide an $\mathrm{n}$ type conductor. The oxygen vacancies maintain overall charge neutrality on the macroscopic scale. The structure of tin oxide can be represented as $\mathrm{SnO}_{2-\mathrm{x}}\left(V_{\ddot{o}}\right)_{\mathrm{x}} \mathrm{e}_{2 \mathrm{x}}\left(0<\mathrm{x}<10^{-5}\right)$. Thus, the conductivity of $\mathrm{SnO}_{2}$ is ascribed to oxygen vacancies that result from its incomplete oxidation.

An alternative explanation of $\mathrm{SnO}_{2}$ conducting properties can be based on the diffusion of $\mathrm{O}^{-2}$ and electron-hopping between metal cations in the metal oxide lattice. In principle, due to non-stoichiometry and oxygen deficiency, the structure of metal oxide semiconducting materials such as $\mathrm{TiO}_{2}$ and $\mathrm{SnO}_{2}$ can be represented as $\mathrm{MO}_{2-\mathrm{x}}\left(0<\mathrm{x}<10^{-5}\right)$, which is a combination of $\mathrm{MO}$ and $\mathrm{MO}_{2}$. The ionic lattice is illustrated in Figure 15: 


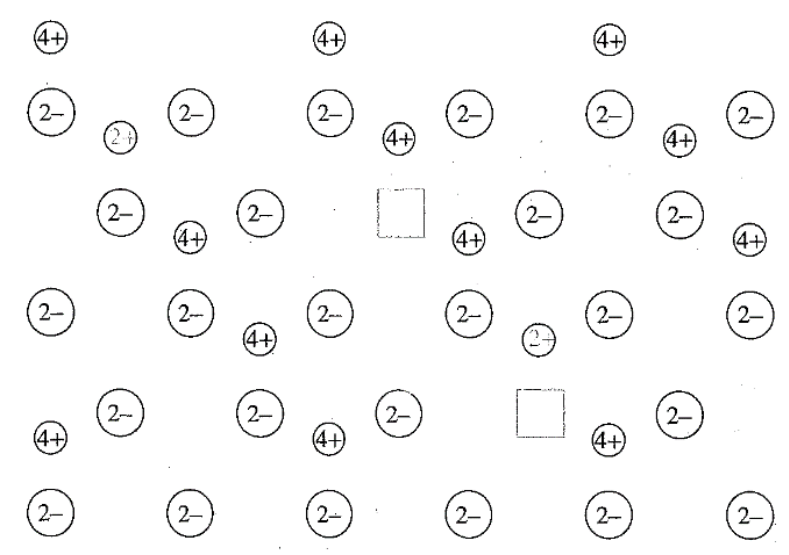

Figure 15: Illustration of ionic lattice of $\mathrm{MO}_{2-\mathrm{x}}$ where small, big circles and squares represent metal cations, oxygen anions and oxygen vacancies, respectively.

As can be seen from Figure 15 , there are a few $\mathrm{M}^{2+}$ ions in a sea of $\mathrm{M}^{4+}$ ions and a few oxygen vacancies in the lattice. Therefore, the conduction can be achieved by the diffusion of $\mathrm{O}^{2-}$ ions through the vacancies or the electrons hopping from $\mathrm{M}^{2+}$ to $\mathrm{M}^{4+}$. The resulting oxide is an n-type due to the domination of electron-hopping [55]

\subsubsection{Raman Active Vibrational Modes of $\mathrm{SnO}_{2}$ Rutile Structure}

Raman spectra of $\mathrm{SnO}_{2}$ can be used to evaluate the effect of doping that enhances the conductivity. A brief discussion of its Raman spectrum is provided in this section.

Since there are 6 atoms in its unit cell, $\mathrm{SnO}_{2}$ lattice structure possesses a total of 18 vibrational modes in the first Brillouin zone. However, just only 4 modes are Raman active including 3 non-degenerate modes $\mathrm{A}_{1 \mathrm{~g}}, \mathrm{~B}_{1 \mathrm{~g}}, \mathrm{~B}_{2 \mathrm{~g}}$ and one doubly degenerate mode $E_{g}$ [56]. Their group theoretical symmetry designations with their irreducible representations are presented in Table 3 below. 
Table 3: Phonon symmetry of $\mathrm{SnO}_{2}$ rutile structure with basic functions of the irreducible representation of Raman active modes. Table is adapted and modified from Ref [57]

\begin{tabular}{|c|c|c|c|}
\hline Phonon Symmetry & Activity & Raman peak position & Irreducible representation \\
\hline $\mathrm{A}_{1 \mathrm{~g}}$ & Raman Active & $634 \mathrm{~cm}^{-1}$ & $\mathrm{x}^{2}+\mathrm{y}^{2}, \mathrm{z}^{2}$ \\
\hline $\mathrm{B}_{1 \mathrm{~g}}$ & Raman Active & $123 \mathrm{~cm}^{-1}$ & $\mathrm{x}^{2}-\mathrm{y}^{2}$ \\
\hline $\mathrm{B}_{2 \mathrm{~g}}$ & Raman Active & $773 \mathrm{~cm}^{-1}$ & $\mathrm{xy}$ \\
\hline $\mathrm{E}_{\mathrm{g}}$ & Raman Active & $473 \mathrm{~cm}^{-1}$ & $\mathrm{zx}, \mathrm{zy}$ \\
\hline
\end{tabular}

In these Raman active modes, oxygen atoms vibrate while $\mathrm{Sn}$ atoms are at rest. Specifically, the non-degenerate modes vibrations take place in the plane perpendicular to the $\mathrm{c}$ axis while doubly degenerate mode vibrates in the direction of the $\mathrm{c}$ axis (Figure 16). The Raman spectrum of rutile-type $\mathrm{SnO}_{2}$ single crystal shows four peaks corresponding to $A_{1 g}\left(634 \mathrm{~cm}^{-1}\right), B_{1 g}\left(123 \mathrm{~cm}^{-1}\right), B_{2 g}\left(773 \mathrm{~cm}^{-1}\right)$ and $E_{g}\left(473 \mathrm{~cm}^{-1}\right) . A_{1 g}$ and $\mathrm{B}_{2 \mathrm{~g}}$ are related to bond expansion and contraction. In the $\mathrm{A}_{1 \mathrm{~g}}$ mode, two Sn-O bonds contract and the other four $\mathrm{Sn}-\mathrm{O}$ bonds expand or contract in opposite ways. In $\mathrm{B}_{2 \mathrm{~g}}$ mode, all six $\mathrm{Sn}-\mathrm{O}$ bonds, which are around the $\mathrm{Sn}$ ion, contract and expand cooperatively, leading to a larger repulsive force of the $\mathrm{O}-\mathrm{O}$ bonds than in $\mathrm{A}_{1 \mathrm{~g}}$ mode. The $\mathrm{B}_{1 \mathrm{~g}}$ mode consists of rotation of the oxygen atoms around the c axis, with all six oxygen atoms of the octahedral participating in the vibration. $E_{\mathrm{g}}$ mode relates to the vibration of oxygen in the direction of the $\mathrm{c}$ axis [58]. 

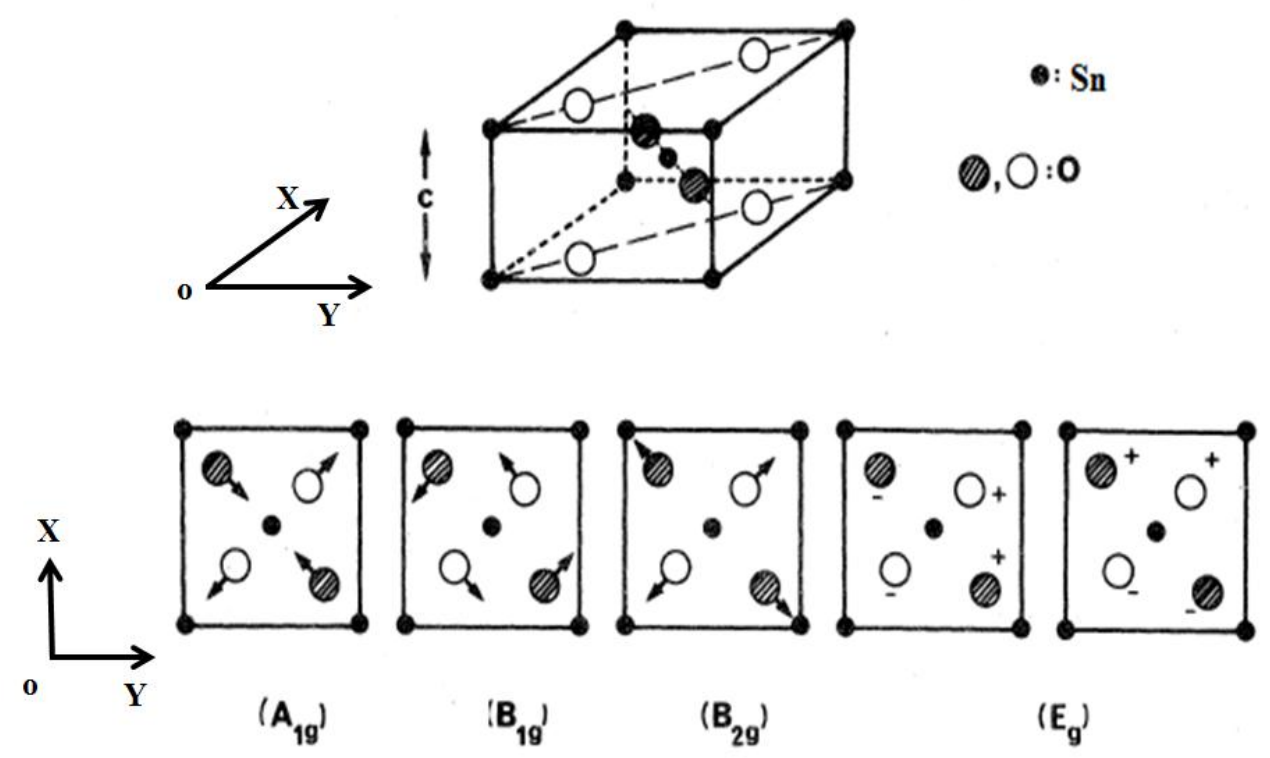

Figure 16: Raman active vibrational modes of $\mathrm{SnO}_{2}$ rutile structure from the projection images in the $\mathrm{x}-\mathrm{y}$ plane [57]. 6 big circles represent 6 oxygen atoms in two different planes (the first plane contains 2 shaded circles and the second contains 4 unshaded circles). Small circles indicate Sn atoms. (+) and (-) signs illustrate the in and out of plane motions, respectively.

\subsubsection{Growth of $\mathrm{SnO}_{2}$ nanowires}

During the last decade, methods of synthesis of $\mathrm{SnO}_{2} \mathrm{NWs}$ have been well established. Different morphologies of $1 \mathrm{D} \mathrm{SnO}_{2}$ have been reported such as nanowires, nanorods, nanobelts and nanotubes. The preparation methods can be classified into two categories: vapor phase deposition and solution-based crystal growth. Many common methods include laser ablation [59, 60], thermal evaporation [61, 62], vapor-liquid-solid (VLS) growth [63, 64], carbothermal reduction [65, 66] and rapid oxidation [67] . The vapor phase deposition methods produce high quality nanostructures with well-defined diameter and length distributions. In the other case, solution based growth techniques use molten salt $[68,69]$ as a solvent to grow nanostructures at high temperature. Furthermore, solvothermal/hydrothermal methods $[62,70]$ provide bulk synthesis at a lower growth temperature. Even though aspect ratios and morphological features are not 
as precisely controlled, as in the case of vapor phase deposition methods, the advantage of solution-based syntheses is bulk scale production to generate materials for sensing application. Therefore, this synthetic pathway was employed in this work to synthesize undoped and doped $\mathrm{SnO}_{2} \mathrm{NWs}$, which are the focus for the sensing application in this chapter.

In the most general sense, the growth mechanism of one-dimensional nanostructures through solution based synthetic methods follows the Oswald ripening process assisted by anisotropic-directing growing factors. In particular, the precursors of crystal growth process are nanoscopic morphologies or seeds. They are formed under supersaturated condition in the dissolved in a medium due to critical conditions such as pressure concentration and temperature [71]. Following this nucleation stage is the crystal growth phase where the Oswald ripening mechanism prevails. During Oswald ripening the larger crystal structures grow at the expenses of smaller crystals [72]. During this process, morphologies of crystal structure formation can be controlled by varying the parameters in the reaction systems that affect the kinetic and theromodynamic properties of the system through choices of solvents, capping reagents, control of reaction temperatures, change of $\mathrm{pH}$ values, etc [73].

Of these, the most important factor in controlling the formation of one dimensional nanostructures is the coordination of surfactants/capping agents [73]. Selective adsorption of surfactant in a particular single crystal plane affects its surface energy. According to the Gibb- Wulff facet theorem, a nanocrsytal, which is bounded by facets, has a minimum total surface energy at equilibrium [74]: 
$\int_{i}^{n} \gamma_{i} d S_{i}=0$

Where:

$\gamma(\mathrm{i})$ : the specific surface free energy of facet $\mathrm{i}$

S(i): surface area of facet $\mathrm{i}$

In other words, the equilibrium shapes of a nanocrsytal are constructed by the facets and their corresponding areas so that the total surface energy is minimized. The morphology of nanostructures is determined by the relatively specific surface energies of the facets. Thus, it is possible manipulate the shape of a crystal by controlling free energies of crystallographic surfaces [75]. The introduction of appropriate surfactants or capping agents can be employed for this purpose during the crystal growth processes through the facet selectively passivating process. Therefore, by appropriately choosing the surfactant, solution based method could be employed to synthesize different one dimensional nanostructure materials [73]. Recently a new mode of NW growth through development screw dislocations has been established [76]. The establishment of this mechanism requires sophisticated TEM analysis and hence was not pursued.

\subsection{5 $\mathrm{SnO}_{2} \mathrm{NWs}$ as sensor material}

As discussed before, oxygen vacancies transform $\mathrm{SnO}_{2}$, a wide band gap semiconductor, to an n-type semiconductor. $\mathrm{SnO}_{2}$ based sensors function through changes in conductivity brought about by electron transport to/from analyte to the semiconductor. There are two kinds of sensing responses observed. The first mechanism is that the electrons are withdrawn from the conduction band upon the adsorption of charge accepting molecules such as $\mathrm{NO}, \mathrm{Cl}_{2}$ and $\mathrm{O}_{2}$ (oxidizing agents). The negatively 
charged adsorbed species repel the majority carriers from the surface. This leads to the depletion region near the surface that is devoid of carriers, i.e., electrons with concomitant reduction of conductivity. On the other hand, as a second mechanism, some molecules such as $\mathrm{CO}$ or $\mathrm{H}_{2}$ (reducing agents) could react with the surface adsorbed oxygen anion and release electrons to the conduction band to cause an increase in conductance [77] by driving the semiconductor in accumulation mode.

Figure 17 below illustrates the adsorption of chlorine gas on the surface of $\mathrm{SnO}_{2}$ nanoparticles. The respective energy levels with respect to vacuum and the Nernst hydrogen electrode levels are shown. Since the redox energy level of $\mathrm{Cl}_{2}$ is lower than oxygen, $\mathrm{Cl}$ - ions can be generated even in the presence of adsorbed oxygen anions. As $\mathrm{Cl}$ - ions are formed by the abstraction of electrons from the conduction band, they repel the majority carriers which are electrons in the n-type semiconductor from the gas-solid interface, leading to formation of a depletion layer as showed in Figure 18. As a result, this layer provides an inter-nanoparticle potential barrier that hinders electron transport and hence increases the sensor resistance [48]. An oxidizing species increases the sensor resistance while a reducing species decreases it resistance for an n-type semiconductor. Complementary behavior is expected for a p-type semiconductor. This generic response can be made selectively if a proper dopant with specific affinity for the analyte can be found. An example for this type was demonstrated in case of $\mathrm{Cl}_{2}$ detection using antimony doping [34] (Figure 17 B). 


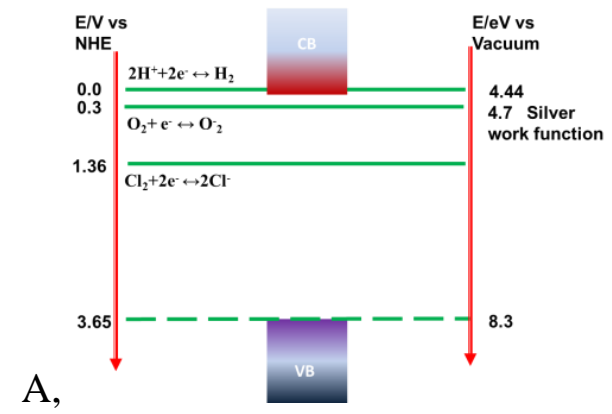

$\mathrm{B}$,

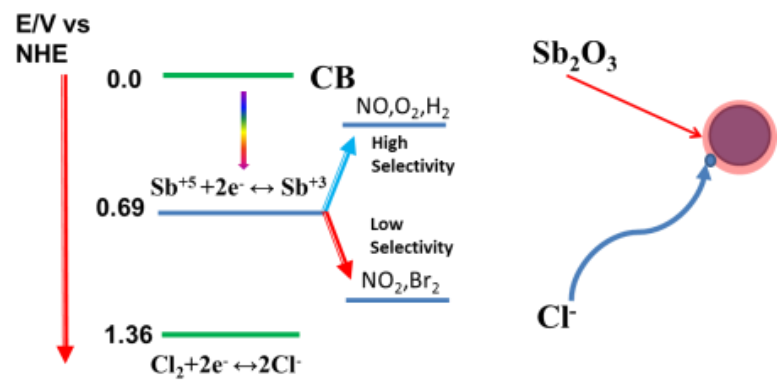

Figure 17: A. Energy levels of oxidizing and reducing gases in relation to band edges of $\mathrm{SnO}_{2}$. B. Location and position $\mathrm{Sb}$ reduction potential which enable it to act as a catalyst/dopant conferring selectivity for $\mathrm{Cl}_{2}$ detection.

Primary driving force for detection of oxidizing species is the location of conduction band edge of n-type $\mathrm{SnO}_{2}$. Any oxidizing species whose reduction potential is below the edge can extract electrons and reduce the conductivity of the $\mathrm{SnO}_{2}$. While reducing species, whose reduction potential is above the filled states of the conduction band, can donate electrons and increase conductivity of the semiconductor. This is dictated by the minimization electron energy which requires that it seeks the lowest available energy state. Given that the reduction potential of antimony is between the conduction band edge and $\mathrm{Cl}_{2}$ reduction potential, the latter is highly and selectively detected with respect to other gases such as $\mathrm{NO}$ and $\mathrm{H}_{2}$ whose reduction potential are greater than antimony. While gases such as $\mathrm{NO}_{2}$ and $\mathrm{Br}_{2}$ are detected but with lower degree of selectivity as their reduction potential falls between chlorine and antimony, 


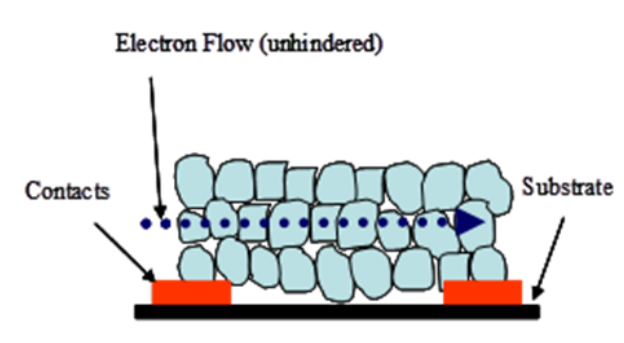

a) $\mathrm{Air}$

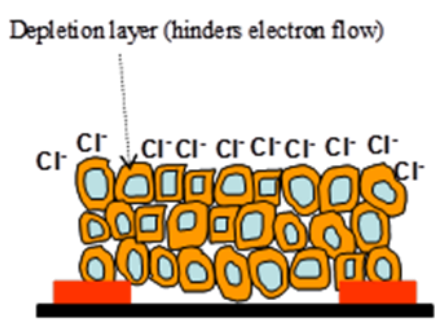

b) Chlorine

c) Chlorine

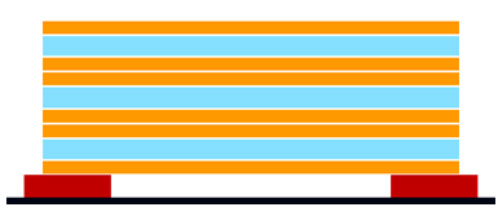

Figure 18: Schematic diagrams of porous sensing layers. a) No or very small depletion layer (high conductivity) in absence of $\mathrm{Cl}_{2}$, (b) in chlorine, depletion layer thickness increases. (c) In nanowires, the number of electron hops amongst the charge carrying particles would be reduced leading to an expectation of lower resistance.

Additionally it can be surmised from Figure $18 \mathrm{~b}$ and 18c, that NWs based sensor should have lower resistance than its counterpart composed from NP as fewer interparticle electron hops are required for electron transport between electrodes. Nevertheless, the actual relative resistance between the NP-based and NW-based sensor might be different. For compressed powders, used in these studies, it is clear that the electrical conductivity of as-fabricated sensors not only depends on extrinsic factors such as the applied pressure but also on the intrinsic factors such as morphological properties [78]. The difference in morphologies, which determine the degree of packing of the material or the density, leads to the change in contact resistance between NWs and NPs in compressed powder. Since the same preparation method was employed the extrinsic factors can be neglected. Intrinsic factors play an important role in the conductivity of asfabricate sensors. These effects are discussed in later in the discussion section. Furthermore, it should be noted that the contacting electrode material, silver, has its 
Fermi level near the conduction band edge, Figure 17 A. This leads to a desirable Ohmic contact for a resistive sensor.

\subsection{Results and Discussion}

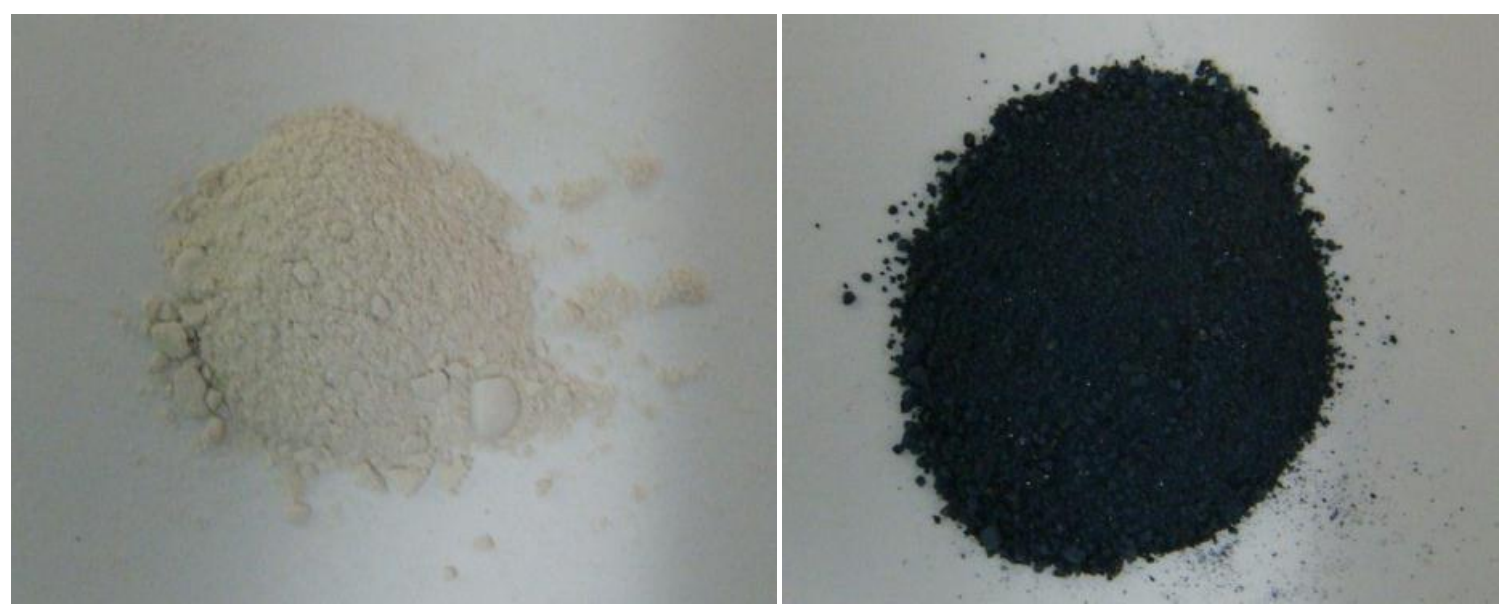

Figure 19: Optical images of undoped (left) and Sb doped NWs (right)

\subsubsection{Electron Microscopy}

A. n-doped $\mathrm{SnO}_{2} \mathrm{NWs}$

The colors of undoped and doped products are illustrated in Figure 19. Different colors white and dark green correspond to undoped and n-doped nanowires. The green color becomes darker for higher dopant concentrations.

The morphology of as-prepared un-doped and Sb-doped $\mathrm{SnO}_{2} \mathrm{NWs}$ was characterized by SEM. The majority of the un-doped NWs showed an average NW diameter ranging from 20 to $50 \mathrm{nms}$ and the length from 1 to $5 \mu \mathrm{ms}$, (Figure 20 left). It was noticed that NWs were mixed with the whisker-like nanorods. In the n-doped NWs, the size distribution was much broader (from a few tens of nanometers corresponding to length of a nano-whisker up to $10 \mu \mathrm{ms}$ for the nanowire length), perhaps due to incorporation and morphological effects of dopant atoms. Also, note that the smaller 
diameter NWs tended to fuse with one another (Figure 20 right). Such structures are rarely observed in the nucleation and growth of NWs mediated by the vapor-liquid solid (VLS) mechanism. TEM and high resolution TEM of n-doped NWs along with electron diffraction in inset (Figure 21) indicate the single crystalline nature of NWs with a prominent peak corresponding to the interplanar spacing of $0.34 \mathrm{~nm}$ reflecting a wellknown crystallographic plane [110] of $\mathrm{SnO}_{2}$. The crystal structure of these $20 \mathrm{~nm}$ diameter NWs was a characteristic cassiterite type (details are not shown here) which is commonly observed upon calcination at $500^{\circ} \mathrm{C}$ in $\mathrm{SnO}_{2} \mathrm{NPs}$ synthesized the by sol-gel method [79]. The NWs showed a very straight and smooth surface morphology suggesting a 1-D Ostwald-ripening mechanism [68] by which the formation of NWs (the larger crystal structures) could have grown at the expense of NPs (smaller crystals). Since the synthesis was carried out at elevated temperature $\left(750^{\circ} \mathrm{C}\right), 1,10$-phenanthroline has a very minor effect as a surface free energy controller on the growth mechanism. However, it is a crucial factor as an interfacial capping agent in the formation of precursors. During the synthesis of NPs, 1,10-phenanthroline could cap to the surface of Sn NPs and prevent their agglomeration as well as the formation of $\mathrm{SnO}_{\mathrm{x}}$. The Sn NPs can grow in spherical shape and more uniform size distribution [80], resulting in better morphology of NWs. This is supported by the experiments summarized in Table 4. Specifically, the higher aspect ratio of $\mathrm{SnO}_{2} \mathrm{NWs}$ was achieved with the presence of 1,10 phenanthroline in the same synthetic condition of temperature and medium. Perhaps, during 1-D Ostwaldripening process, this capping agent has reduced the formation of $\mathrm{SnO}_{\mathrm{x}}$ on the surface of Sn NPs and facilitated the growth of $\mathrm{SnO}_{2}$ NWs. 

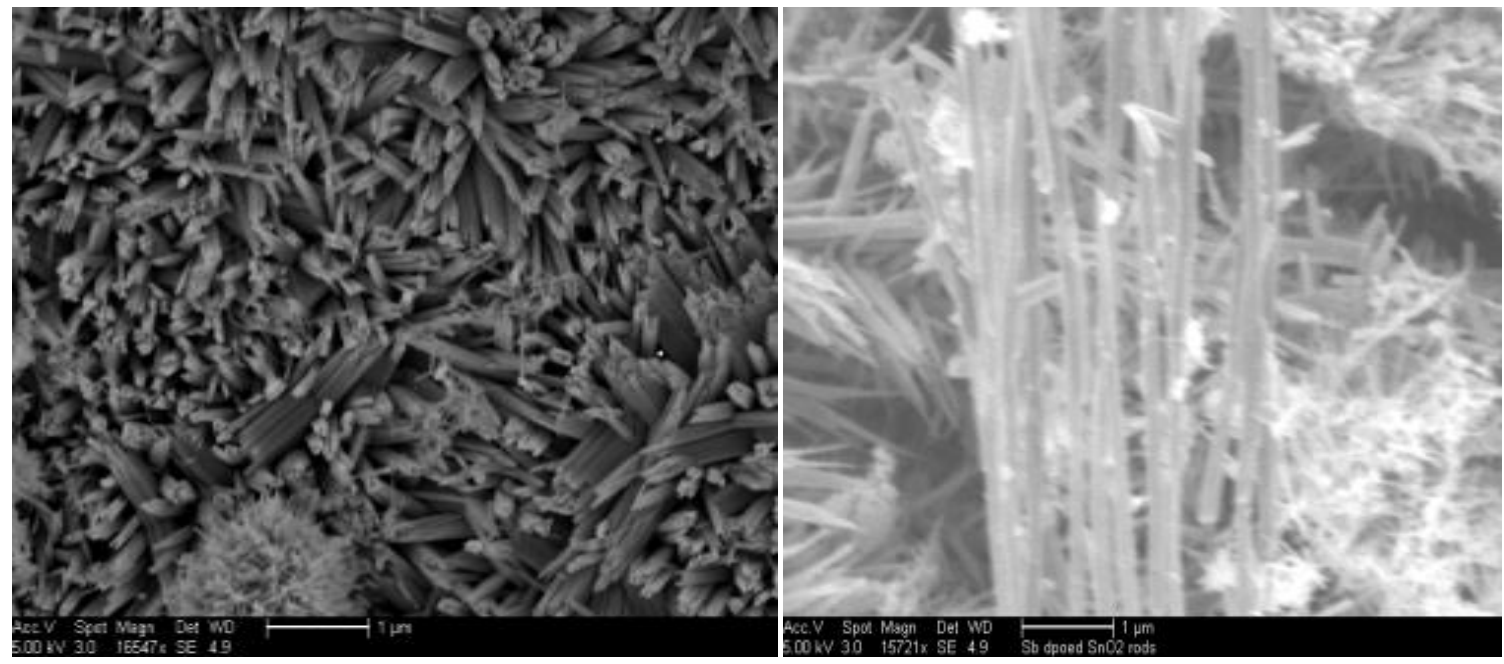

Figure 20: SEM images of undoped (left) and Sb doped NWs (right)

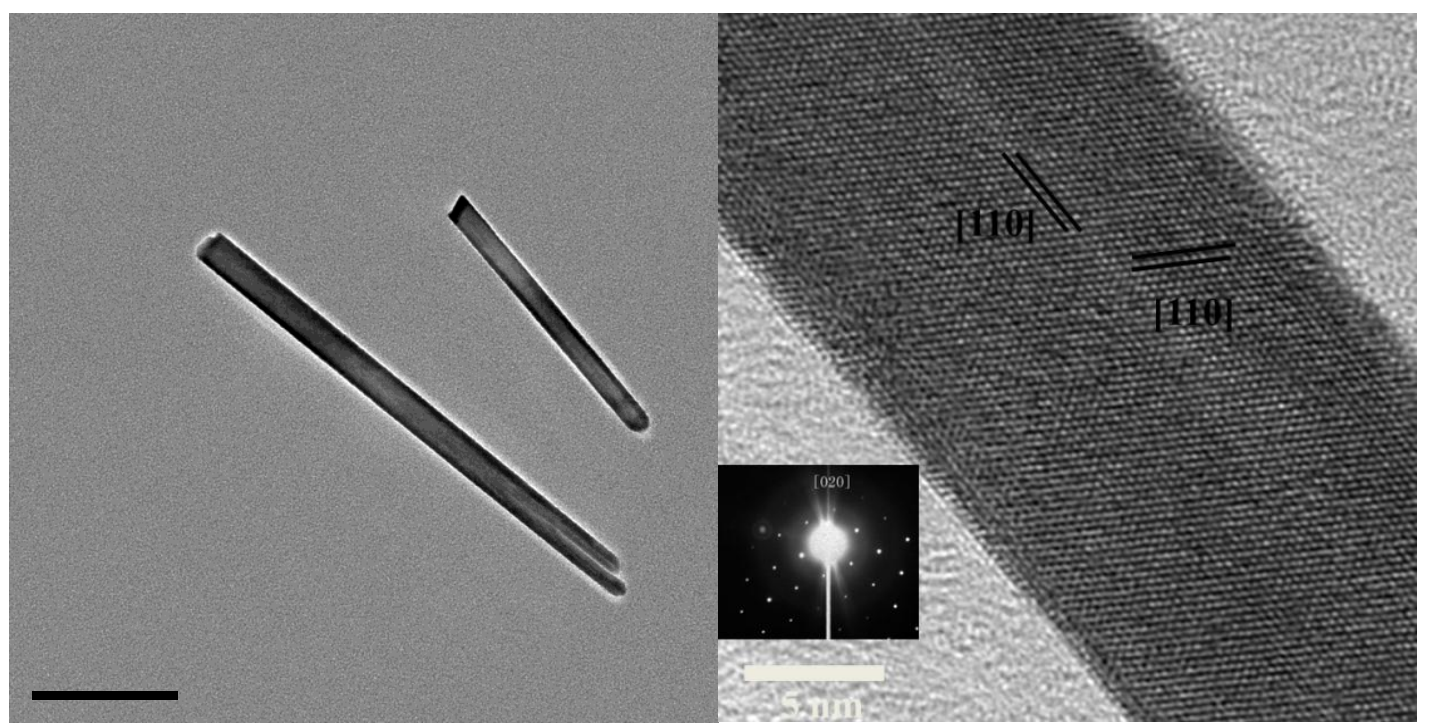

Figure 21: TEM images of n-doped $\mathrm{SnO}_{2} \mathrm{NWs}$ with low (left) and high magnifications along with electron diffraction in the inset (right).

\section{B. Li-dopedSnO 2 NWs:}

The color of Li-doped nanowires is more yellowish than undoped nanowires as showed in Figure 22. 


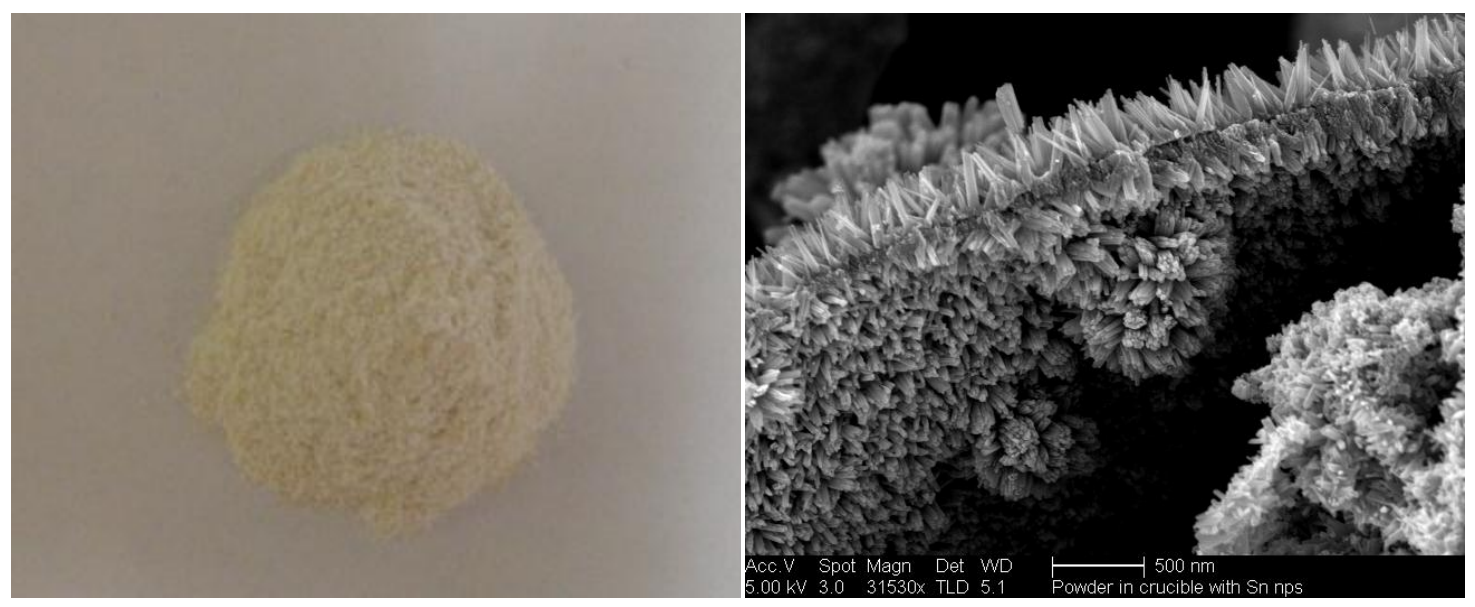

Figure 22: Optical image and SEM image of Li-doped $\mathrm{SnO}_{2} \mathrm{NWs}$ synthesized at $490^{\circ} \mathrm{C}$ SEM images of Li-doped NWs synthesized at $490^{\circ} \mathrm{C}$ showed a broad size distribution for length and diameter. The length of wires varied from $200 \mathrm{~nm}$ up to $1 \mathrm{um}$ while the diameter of single wires ranged from $50 \mathrm{~nm}$ to hundreds of nanometers. Like the n-doped NWs, whisker-like nanorods with smaller diameters and shorter lengths were also observed along with the tendency of the NWs to fuse. Thus, preformed NPs transformed into NWs through recrystallization from alkali halide salts exhibited similar characteristics regardless of the nature (n or $\mathrm{p}$ type) of the dopant. The morphology of NW nanostructure was influenced by a variety of factors, such as precursors, the moisture content of grounded mixture before the recrystallization and growth temperature. Different experiments (Table 4) have been carried out to investigate the effects of synthetic conditions on the formation of $\mathrm{SnO}_{2} \mathrm{NWs}$. Results indicated that with the same synthetic conditions, better morphology in terms of the aspect ratio of $\mathrm{SnO}_{2} \mathrm{NWs}$ product were achieved by using $\mathrm{Sn}$ nanoparticles compared to their oxide capped counterparts. Besides, it was observed that the pre-synthesized powder, when dried to get rid of moisture, gave a better yield of wires and more uniform dimensional distributions 
(data not shown here). Also, the NWs formed at temperatures higher than $490^{\circ} \mathrm{C}$, gave longer wires (>1um in length) with a more uniform length distribution as shown in Figure 23. Replacing coated Sn NP precursors by SnO NPs [81] did not produce NWs at $490^{\circ} \mathrm{C}$. However, recrystallization at $750^{\circ} \mathrm{C}$ did produce NWs with low yield.
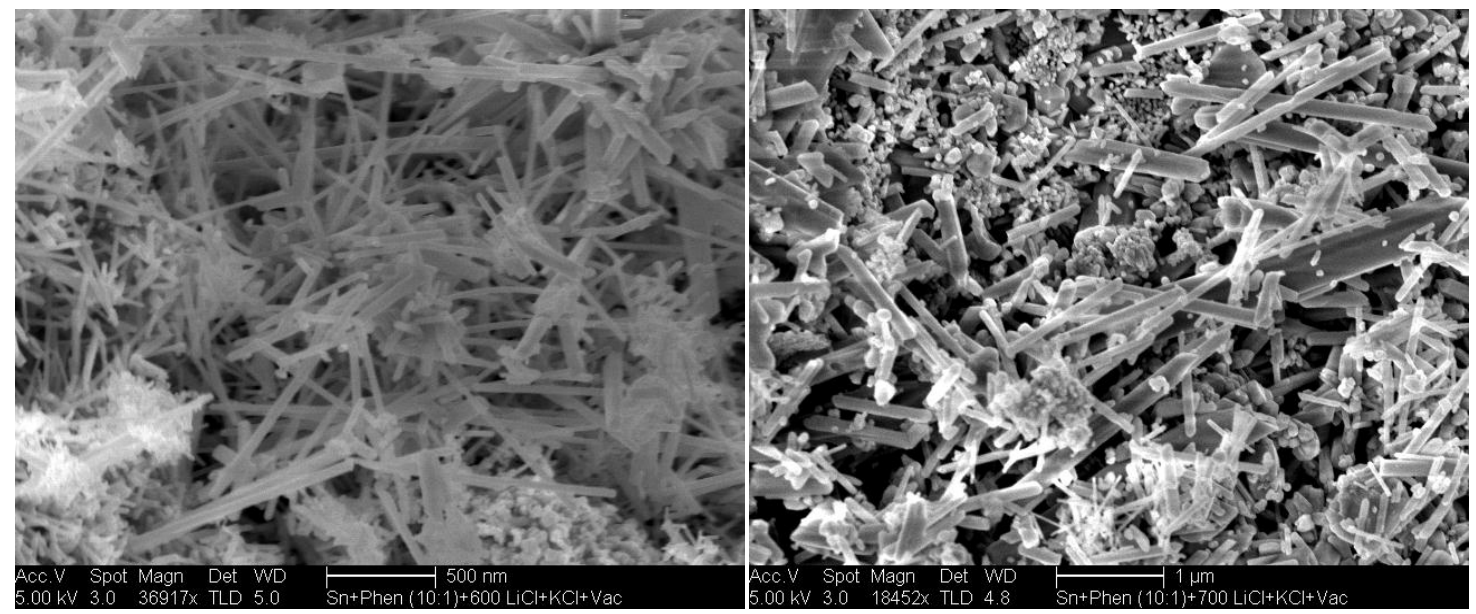

Figure 23: SEM image of Li-doped $\mathrm{SnO}_{2} \mathrm{NWs}$ synthesized at $600^{\circ} \mathrm{C}$ and $700^{\circ} \mathrm{C}$

Table 4: The effect of synthetic conditions on $\mathrm{SnO}_{2} \mathrm{NWs}$ morphology (O and $\mathrm{NO}$ stand for observed and non-observed, respectively)

\begin{tabular}{|l|l|l|}
\hline Synthetic condition of $\mathrm{SnO}_{2} \mathrm{NWs}$ & Length $(\mathrm{nm})$ & Diameter $(\mathrm{nm})$ \\
\hline $\mathrm{SnO}_{\mathrm{x}} \mathrm{nps}+\mathrm{N}_{2}+490^{\circ} \mathrm{C}$ & $\mathrm{NO}$ & NO \\
\hline $\mathrm{SnO}_{\mathrm{x}} \mathrm{nps}+\mathrm{N}_{2}+\mathrm{Phen}+490^{\circ} \mathrm{C}$ & $\mathrm{O}$ & $20 \pm 2$ \\
\hline Dilute $\mathrm{SnO}+490^{\circ} \mathrm{C}$ & 925 & $125 \pm 6$ \\
\hline $\mathrm{Sn} n p s+\mathrm{N}_{2}+490^{\circ} \mathrm{C}$ & $240 \pm 3$ & $46 \pm 5$ \\
\hline $\mathrm{Sn} n p s+$ Air $+490^{\circ} \mathrm{C}$ & $292 \pm 3$ & $21 \pm 3$ \\
\hline $\mathrm{Sn}+$ Air $+\mathrm{Phen}+490^{\circ} \mathrm{C}$ & $407 \pm 4$ & $129 \pm 3$ \\
\hline $\mathrm{Sn}$ nps + Air $+700^{\circ} \mathrm{C}$ & $800 \pm 20$ & $72 \pm 2$ \\
\hline
\end{tabular}

In addition, HR-TEM was also employed to characterize the precusors and resulting NWs. SnO coated Sn NPs were synthesized by methods B and C, described in chapter 2 (section 2.1.1.1). Figure 24 shows the morphology of nanoparticles with 
spherical shape and mean diameter around $10 \mathrm{~nm}$. They show a tendancy to agglomerate perhaps due to the absence of interfacial capping agent 1,10 phenanthroline.

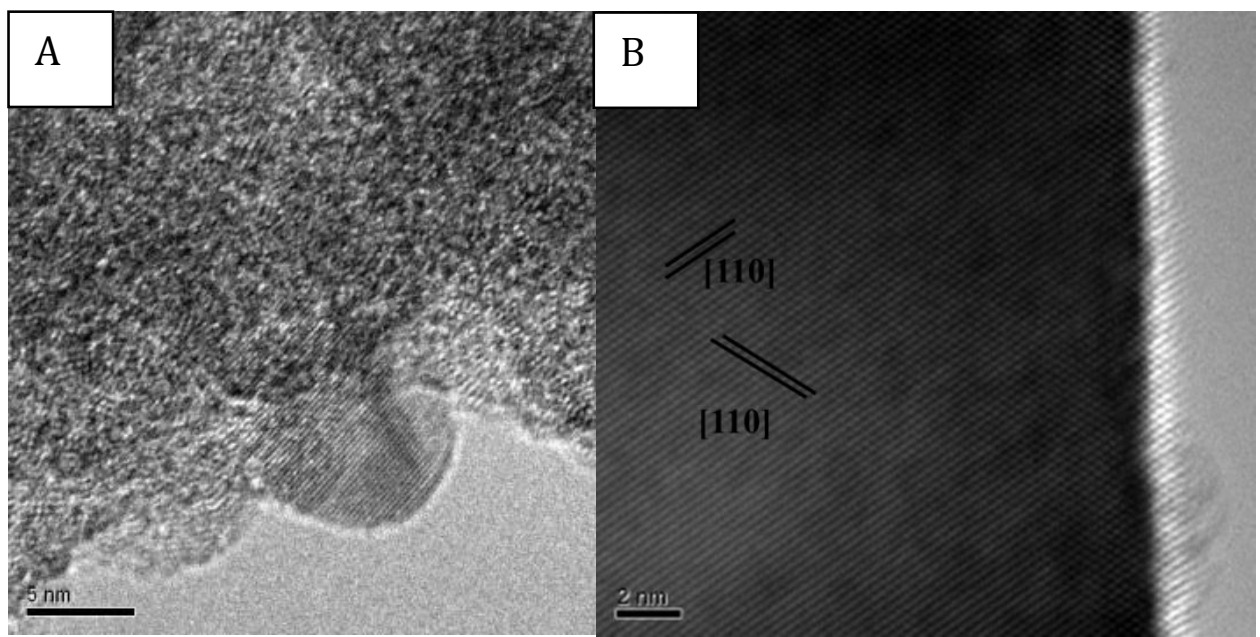

Figure 24: A) HR-TEM of $\mathrm{SnO}_{2-\mathrm{x}}$ coated $\mathrm{Sn} \mathrm{NP}$ precursors and B) TEM of Li-dopedSnO $\mathrm{NWs}_{2}$

The agglomeration of NPs was possibly responsible for fused NWs during the growth process (Figures 20, 22 and 23). The HR-TEM of Li-doped $\mathrm{SnO}_{2} \mathrm{NWs}$ shown in Figure 24B indicated their single crystalline nature with lattice fringe characteristics. The lattice interplanar spacing is about $0.34 \mathrm{~nm}$ corresponding to the [110] plane of the cassiterite crystal structure.

\subsubsection{Photoluminescence Characterization}

$$
\text { A. n-doped } \mathrm{SnO}_{2} \mathrm{NWs:}
$$

Room-temperature photoluminescence spectra of undoped $\mathrm{SnO}_{2} \mathrm{NPs}$ and NWs appear in Figure 25. The excitation wavelength used in this study was $310 \mathrm{~nm}$ corresponding to energy greater than the bulk bandgap energy for $\mathrm{SnO}_{2}$. Two different PL characteristics were observed for NPs and NWs. Both nanostructures gave a UV emission at 360nm, corresponding to the band gap of $\mathrm{SnO}_{2}(3.56 \mathrm{ev})$, partially overlapped 
with Raman (stokes shift) scattering of water (this also occurs at $360 \mathrm{~nm}$ ). The latter peak appears at a constant frequency different from the excitation frequency.

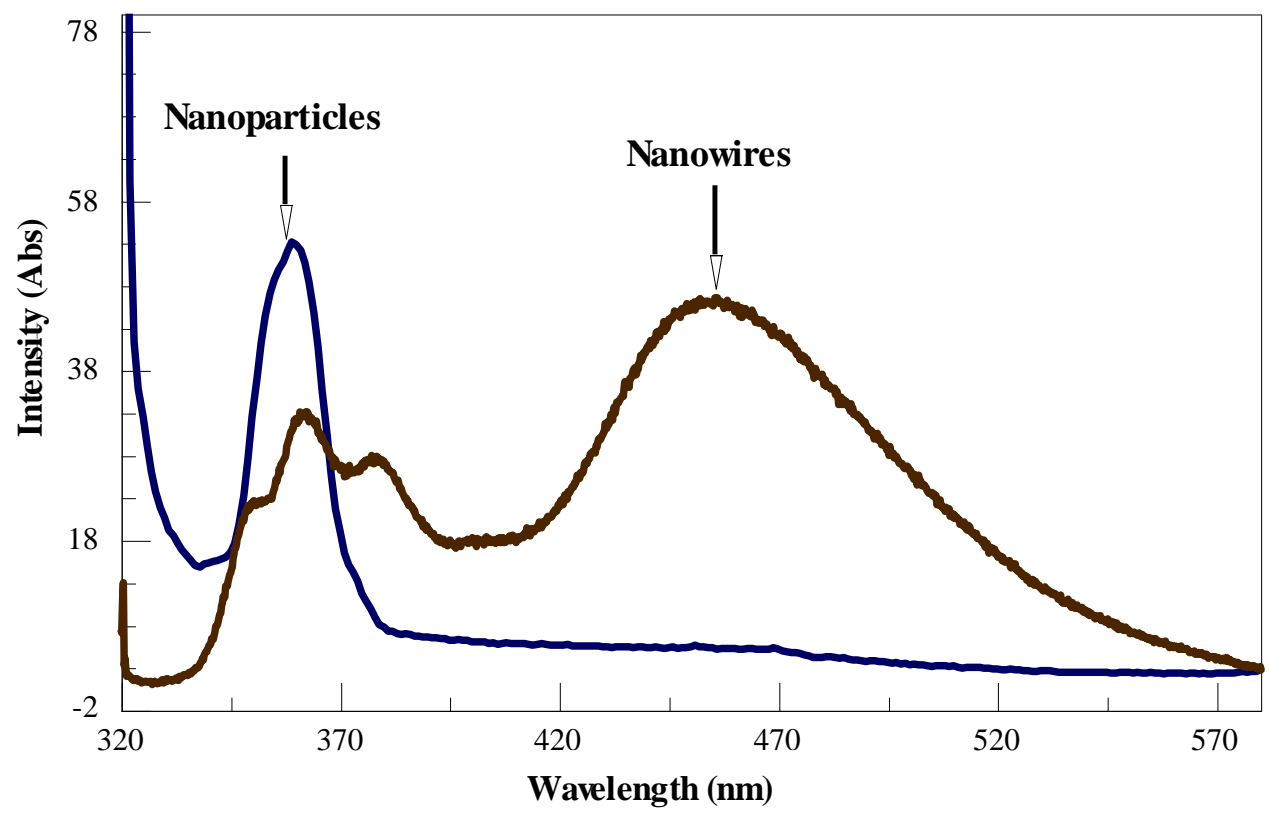

Figure 25: Photoluminescence of undoped $\mathrm{SnO}_{2} \mathrm{NWs}$ and NPs (excitation wavelength $310 \mathrm{~nm}$ ). A new broad peak around $460 \mathrm{~nm}(2.7 \mathrm{eV})$ was observed for NWs.

Bulk $\mathrm{SnO}_{2}$ is an indirect band-gap semiconductor so it is not expected to have a strong PL corresponding to its bandgap energy, as it would violate the principle of momentum conservation. Observation of PL in these nano-geometries (i.e. in both NPs and NWs) implies a PL characteristic of a direct band-gap type semiconductor. Similar effects are well known in silicon NPs, porous silicon and silicon NWs [82] but are not still well understood. Interestingly, the NWs have an additional broad emission peak near 460nm $(2.7 \mathrm{eV})$. The precise understanding of this PL peak currently does not exist; although explanations in terms of defects including vacancies of oxygen, dopant segregation, and lattice disorders inside the lattice of $\mathrm{SnO}_{2}[60,68,83,84]$ or near the surface can be advanced. 
High concentration of dopants can lead to an impurity band near the conduction (in the case of n-type dopant) or the valence (in the case of Li dopant) bands. The resulting band gap narrowing could result in a red shift in the PL. As shown below in Figure 26, under the same excitation wavelength $280 \mathrm{~nm}$, both un-doped and $\mathrm{n}$-doped $\mathrm{SnO}_{2} \mathrm{NWs}$ gave two UV emission peaks, one, at $310 \mathrm{nms}$, is due to solvent Raman scattering and the other broad peak appearing at $\sim 345 \mathrm{~nm}$ wavelength $(3.59 \mathrm{eV})$. For the latter peak a red shift of $20 \mathrm{~nm}$ was observed for Sb-doped NWs, with peak $\sim 365 \mathrm{~nm}$ (3.4eV), in comparison with the undoped NWs. This is consistent with a dopant induced band-gap narrowing concept [85]. These estimate give an approximate bandgap which is larger than the actual bandgap as the precise method for determining band gap is unavailable. Using the PL band edge at high wavelength can have contribution from midgap or surface states so its use would report lower band gap, hence the emission peak maximum was chosen as a crude compromise.

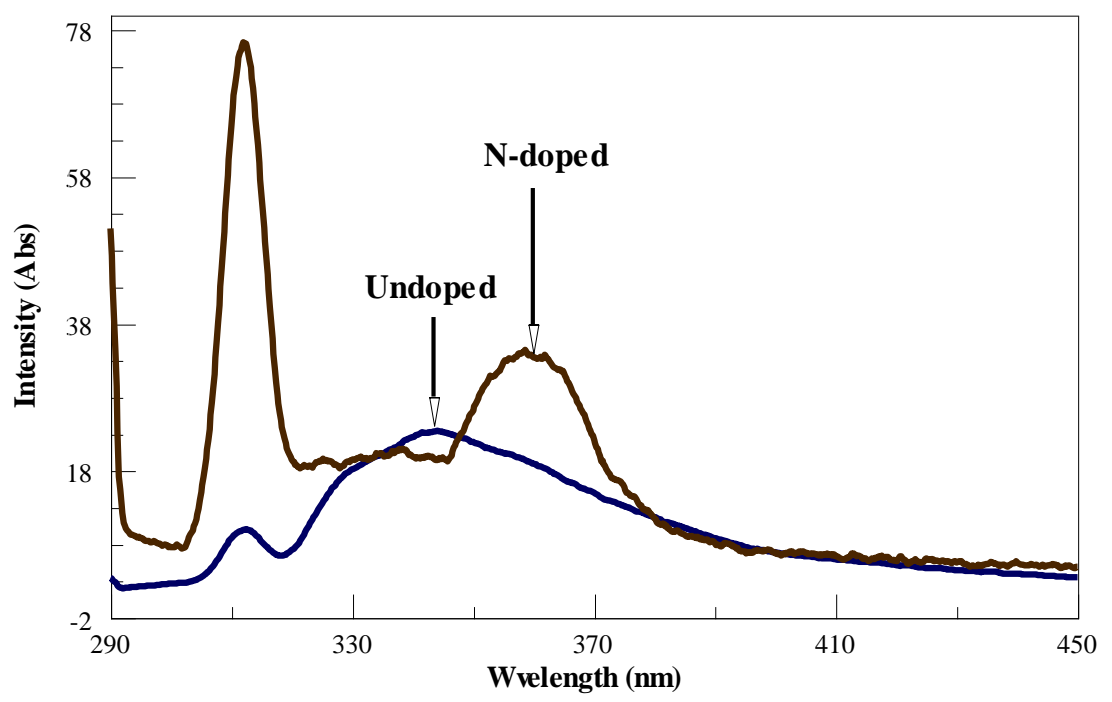

Figure 26: Photoluminescence of undoped and n-doped $\mathrm{SnO}_{2} \mathrm{NWs}$ (excitation wavelength $280 \mathrm{~nm}$ ). The red shift of $20 \mathrm{~nm}$ is observed. Note Solvent scattering at $310 \mathrm{~nm}$ 


\section{B. Li-doped $\mathrm{SnO}_{2} \mathrm{NWs:}$}

A comparison of photoluminescence spectra, excited with $280 \mathrm{~nm}$, in undoped, $5 \% \mathrm{n}$ - and 5\% Li-doped $\mathrm{SnO}_{2} \mathrm{NWs}$ appears in Figure 27. Two emission peaks were observed for all different types (undoped, n- and Li-doped) of NWs. The first peak at $310 \mathrm{~nm}$ is due to solvent. The PL of Li-doped NWs also showed a red shift ( $\approx 23 \mathrm{nms})$ for the broad emission peak occurring at $\sim 368 \mathrm{nms}(3.37 \mathrm{eV})$. This shift could be explained similarly to the case of n-doped using the induced band-gap narrowing concept.

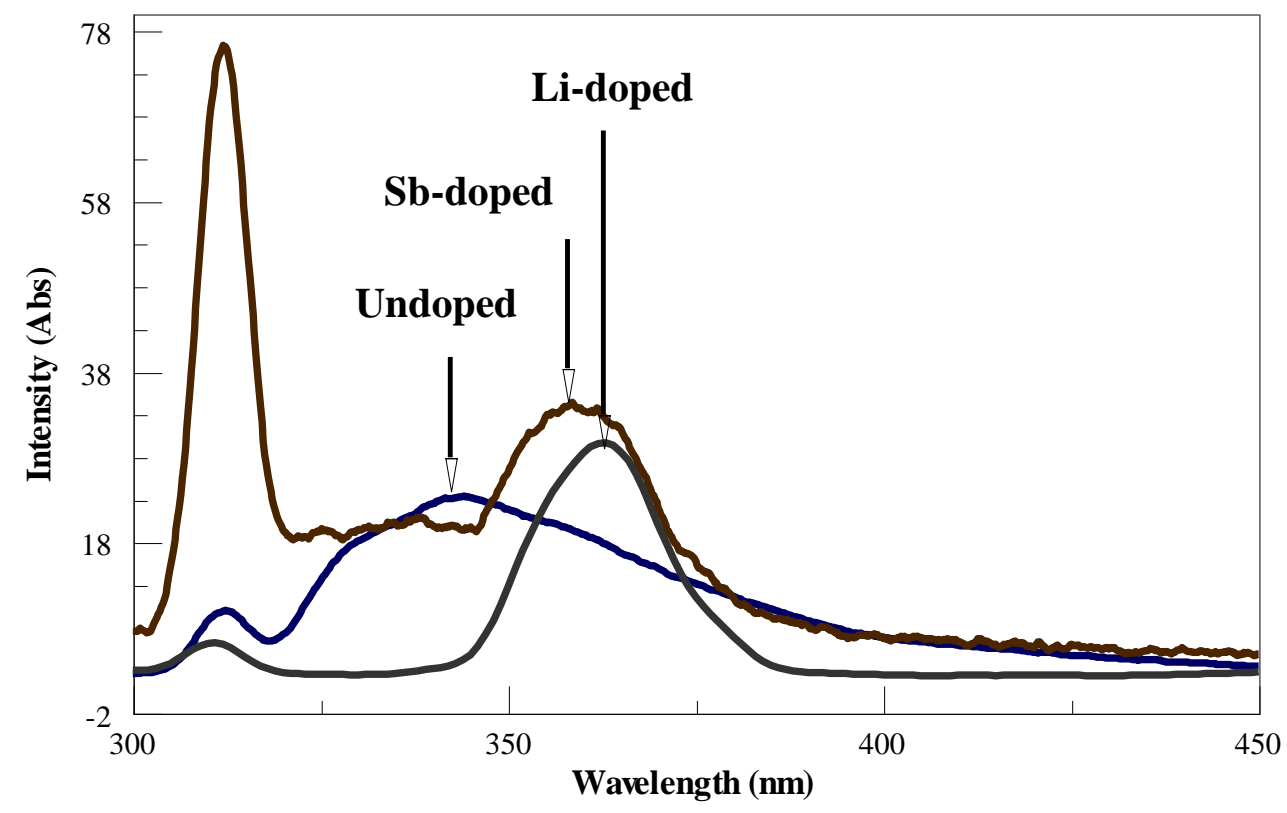

Figure 27: Photoluminescence of undoped (peak $345 \mathrm{~nm}$ or $3.59 \mathrm{eV}$ ), 5\% n-doped (peak at $365 \mathrm{~nm}$ or 3.4 $\mathrm{eV}$ ) and $5 \% \mathrm{Li}$-doped (peak at $368 \mathrm{~nm}$ or $3.38 \mathrm{eV}$ ) $\mathrm{SnO}_{2} \mathrm{NWs}$. Solvent peak is at $310 \mathrm{~nm}$

\subsubsection{UV-Vis Spectroscopy}

The optical bandgap of $\mathrm{SnO}_{2}$ nanostructure samples including undoped, doped $\mathrm{SnO}_{2} \mathrm{NWs}$ and n-doped $\mathrm{SnO}_{2}$ NPs were investigated using the UV-visible absorption spectrometer in the wavelength range of $200-800 \mathrm{~nm}$ as shown in Figure 28. The 
wavelength on the $\mathrm{X}$-axis was converted to energy unit, (eV) for the bandgap determination as following:

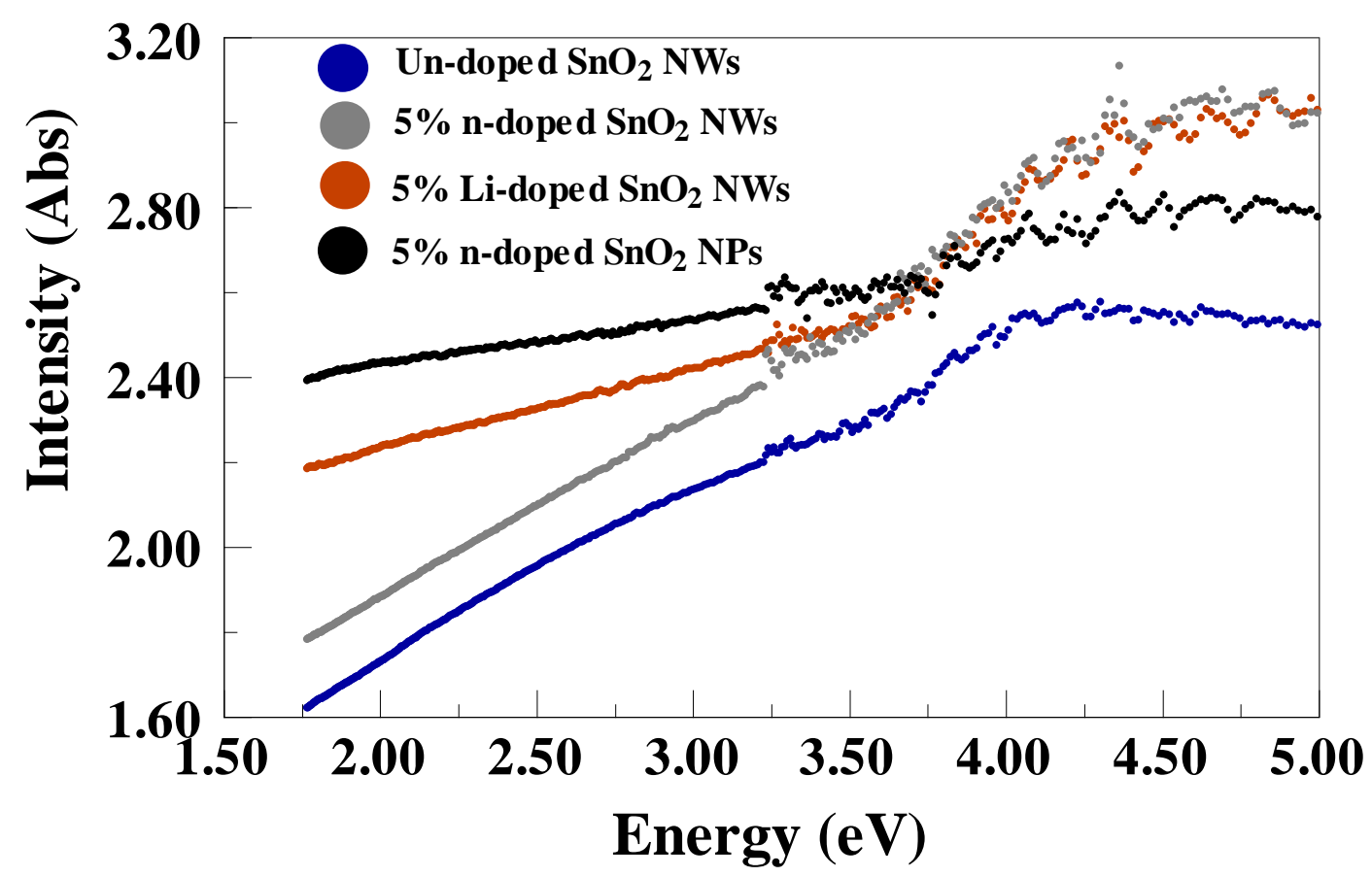

Figure 28: UV-Vis spectra of different $\mathrm{SnO}_{2} \mathrm{NW}$ samples: undoped, 5\% n-doped and 5\% Li-doped. 5\% ndoped NP sample was also investigated for comparison.

It is suggested that the bandgap is direct in the rutiled-nanostructure of $\mathrm{SnO}_{2}$ [86]. As the direct interband transition takes place, the optical absorption coefficient $\alpha$ follows as a function of incident photon energy E exceeding the band gap [87].

$$
\alpha=\left[A\left(E-E_{0}\right]^{\frac{1}{2}}\right.
$$

Where:

$\mathrm{E}_{0}$ is optical bandgap and $\mathrm{A}$ is a constant.

Since the optical absorption coefficient $\alpha$ and transmittance $\mathrm{T}$ are related as:

$$
T=e^{-\alpha L} \text { or } \alpha=-\frac{\ln T}{L}
$$


Where

$\mathrm{L}$ is the thickness of the sample.

Therefore the transmittance and incident photon energy are correlated as:

$$
-\frac{\ln T}{L}=\left[A\left(E-E_{0}\right]^{\frac{1}{2}} \text { or }(\ln T)^{2}=A L^{2}\left(E-E_{0}\right)\right.
$$

This linear relationship is confirmed in the photon energy range of $3.5-4.5 \mathrm{eV}$ depicted in Figure 29, and its extrapolation to the $\mathrm{E}$ axis gives $\mathrm{E}_{0}$ values for different $\mathrm{SnO}_{2}$ samples.
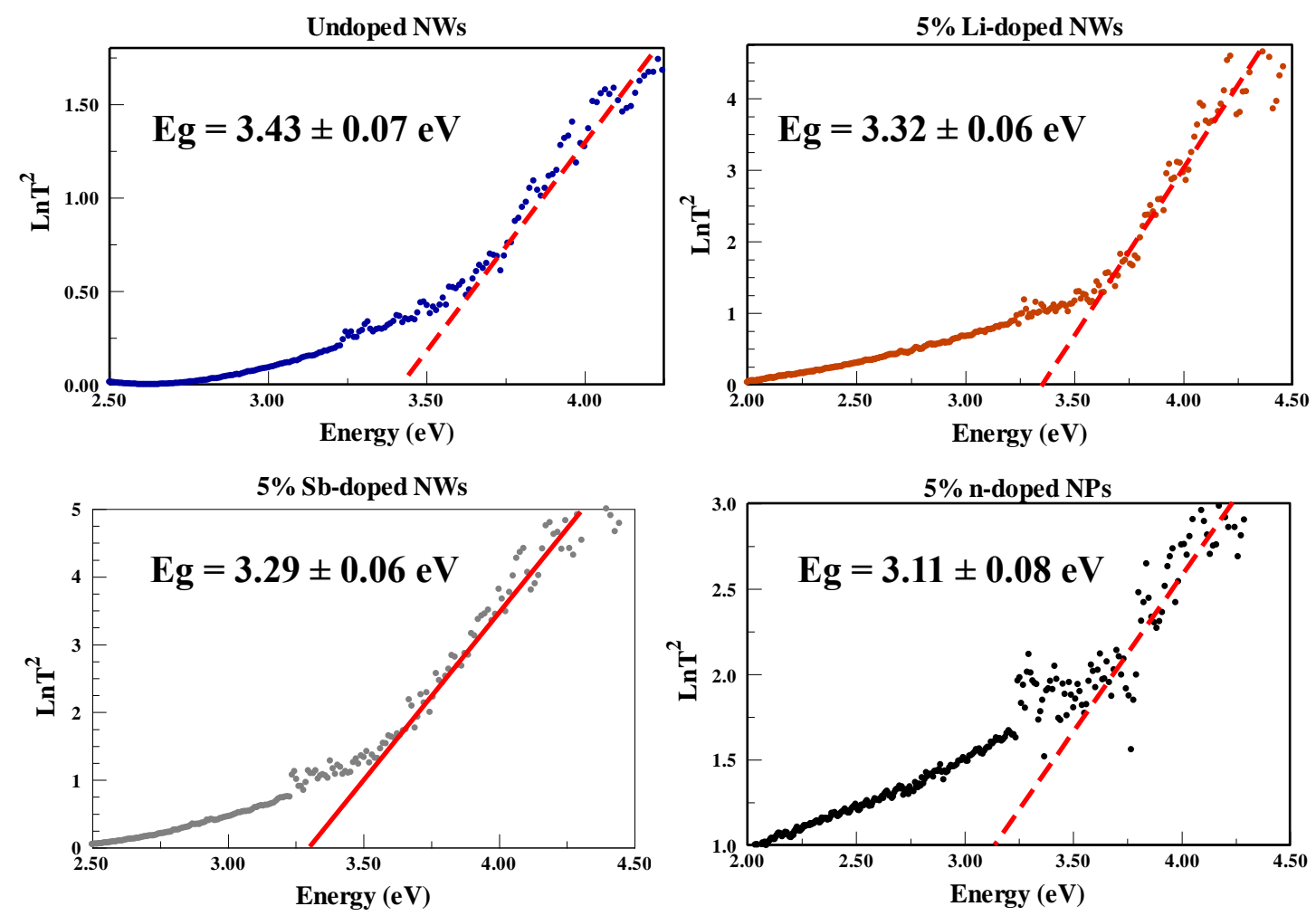

Figure 29: Determination of optical bandgap from the UV absorption edge. Points and dashed curve are experimental and linear fitting/extrapolating, respectively. 
Table 5: Optical bandgap determined from UV absorption edge and photoluminescence

\begin{tabular}{|c|c|c|c|c|}
\hline Sample & $\begin{array}{c}\text { Undoped } \\
\mathrm{SnO}_{2} \mathrm{NWs}\end{array}$ & $\begin{array}{c}5 \% \text { Li-doped } \\
\mathrm{SnO}_{2} \mathrm{NWs}\end{array}$ & $\begin{array}{c}5 \% \text { n-doped } \\
\mathrm{SnO}_{2} \mathrm{NWs}\end{array}$ & $\begin{array}{c}5 \% \text { n-doped } \\
\mathrm{SnO}_{2} \mathrm{NPs}\end{array}$ \\
\hline $\begin{array}{c}\text { Band edge from UV- } \\
\text { VIS } \mathrm{E}_{0}(\mathrm{eV})(\lambda \mathrm{nm})\end{array}$ & $\begin{array}{c}3.43 \pm 0.07 \\
(362)\end{array}$ & $\begin{array}{c}3.32 \pm 0.06 \\
(373)\end{array}$ & $\begin{array}{c}3.29 \pm 0.06 \\
(377)\end{array}$ & $\begin{array}{c}3.11 \pm 0.08 \\
(398)\end{array}$ \\
\hline $\begin{array}{c}\text { Band peak from PL } \\
\mathrm{E}_{0}(\mathrm{eV})\end{array}$ & $3.6 \pm 0.1$ & $3.38 \pm 0.0 .6$ & $3.4 \pm 0.04$ & N/A \\
\hline
\end{tabular}

The evaluated bandgaps of different $\mathrm{SnO}_{2}$ nanostructure samples are summarized in Table 5. The optical bandgap of undoped $\mathrm{SnO}_{2} \mathrm{NWs}$ was determined to be $3.43 \pm 0.07$ $\mathrm{eV}$, which is close to the bandgap of bulk $\mathrm{SnO}_{2} 3.6 \mathrm{eV}$ [88]. Upon the introduction of impurities into the lattice, the bandgap of $\mathrm{SnO}_{2} \mathrm{NWs}$ decreases to $3.32 \pm 0.06 \mathrm{eV}$ and $3.29 \pm 0.06 \mathrm{eV}$ corresponding to $5 \% \mathrm{Li}$-doped and $5 \% \mathrm{n}$-doped, respectively. Specifically, donor and acceptor energy levels have been added by the impurities into the band gap of $\mathrm{SnO}_{2}$ inducing the shift of the Fermi level closer to band edges (CB for ntype and VB for p-type). With an increase in the amount of doping, the density of states generated by impurities increases and forms a continuum of states below or above the conduction or valence bands leading to narrowing of the bandgap [89]. In addition, the decrease of the bandgap upon doping, and determined herein, is consistent with what has been observed from photoluminescence analysis, by which $3.59 \mathrm{eV}, 3.40 \mathrm{eV}, 3.38 \mathrm{eV}$ are the bandgaps of undoped, 5\% n-doped and 5\% Li-doped $\mathrm{SnO}_{2} \mathrm{NWs}$, respectively.

It was observed that with the same doping concentration of antimony, doped $\mathrm{SnO}_{2}$ nanoparticles synthesized from sol-gel methods have a bandgap energy $3.11 \pm 0.08 \mathrm{eV}$ lower than doped $\mathrm{SnO}_{2} \mathrm{NWs}$ synthesized by the molten salt method. This could be the result of different concentration of impurities inserting into the lattice during the doping processes by two different synthetic pathways. However no further quantitative analysis was performed to verify this hypothesis. 


\subsubsection{Raman Spectroscopy}

The Raman of $\mathrm{SnO}_{2}$ nanostructure samples including undoped, doped $\mathrm{SnO}_{2} \mathrm{NWs}$ and doped $\mathrm{SnO}_{2} \mathrm{NPs}$ were investigated using a custom-fabricated Raman system. The data were recorded in the wavelength range $550-850 \mathrm{~cm}^{-1}$ as shown in Figure 30.

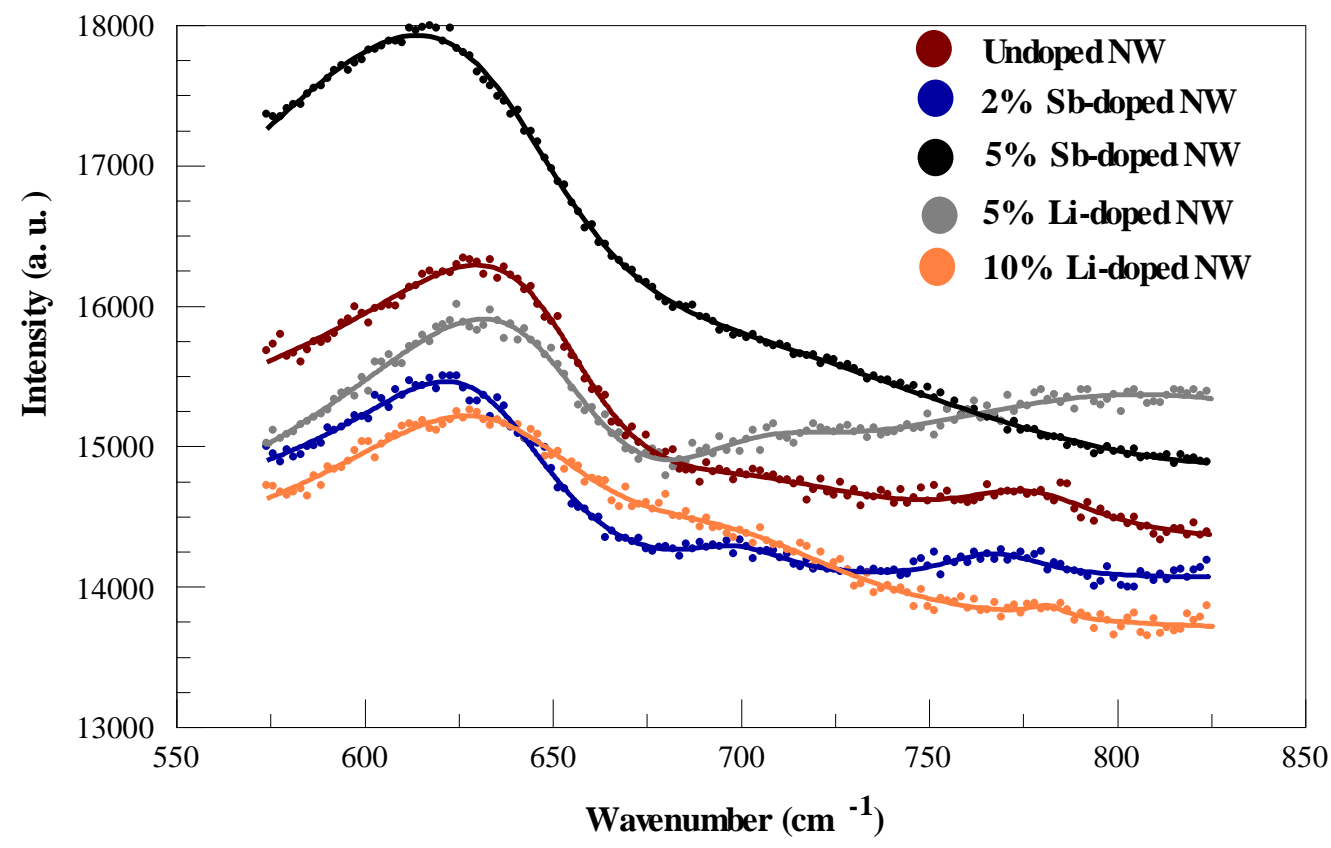

Figure 30: Raman spectrum of $\mathrm{SnO}_{2}$ NWs samples. Three peaks at around $633\left(\mathrm{~cm}^{-1}\right)$ corresponding $\mathrm{A}_{1 \mathrm{~g}}$ symmetry vibration, $697\left(\mathrm{~cm}^{-1}\right)$ and $775\left(\mathrm{~cm}^{-1}\right)$ corresponding to $B_{2 \mathrm{~g}}$ symmetry were observed. Points and solid curve are experimental and fitting, respectively.

Each curve in the spectrum was fitted using three Lorentzians and one tilted baseline. An example of fitting for $2 \%$ n-doped NWs is illustrated in Figure 31. The complete data analysis of Raman peaks from the curve fittings is showed in Table 6 


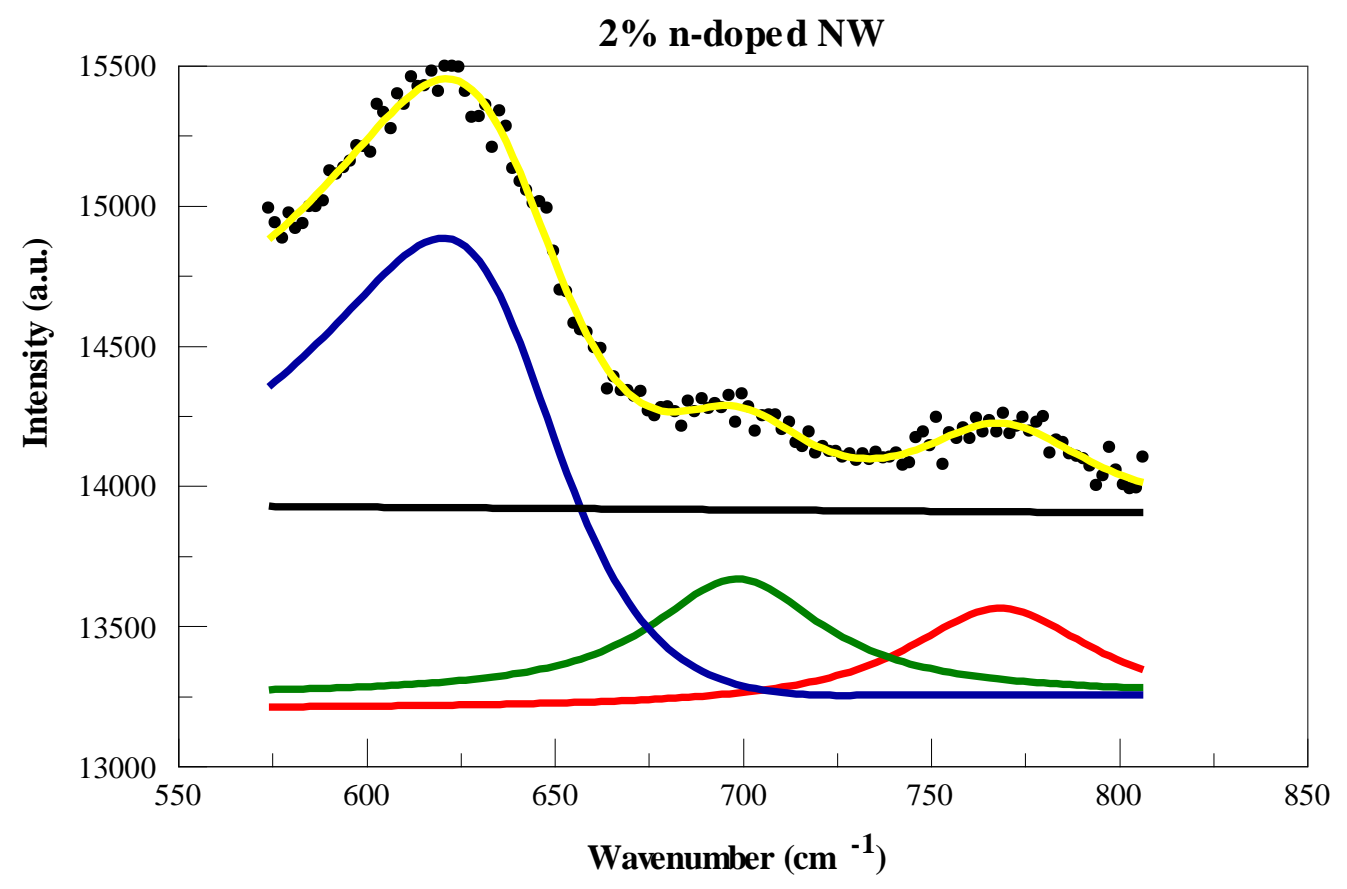

Figure 31: Illustration of curve fitting of $2 \%$ n-doped $\mathrm{SnO}_{2} \mathrm{NWs}$ sample. Points and solid curve (yellow) are experimental and fitting, respectively. The complete fitted curve (yellow) is composed from three Lorentzian (blue, green and red solid) curves and one tilted baseline.

Table 6: Raman data analysis of different $\mathrm{SnO}_{2}$ samples

\begin{tabular}{|c|c|c|c|c|c|}
\hline $\begin{array}{c}\text { Sample/ } \\
\text { vibrational mode }\end{array}$ & $\begin{array}{c}\text { Undoped } \\
\mathrm{SnO}_{2} \mathrm{NWs}\end{array}$ & $\begin{array}{c}2 \% \text { n-doped } \\
\mathrm{SnO}_{2} \mathrm{NWs}\end{array}$ & $\begin{array}{c}5 \% \text { n-doped } \\
\mathrm{SnO}_{2} \mathrm{NWs}\end{array}$ & $\begin{array}{c}5 \% \text { Li-doped } \\
\mathrm{SnO}_{2} \mathrm{NWs}\end{array}$ & $\begin{array}{c}10 \% \text { Li-doped } \\
\mathrm{SnO}_{2} \mathrm{NWs}\end{array}$ \\
\hline $\mathrm{A}_{1 \mathrm{~g}}\left(\mathrm{~cm}^{-1}\right)$ & $632 \pm 2$ & $622 \pm 3$ & $617 \pm 3$ & $627 \pm 6$ & $623 \pm 3$ \\
\hline $\mathrm{A}_{2 \mathrm{u}}\left(\mathrm{cm}^{-1}\right)$ & N/A & $698 \pm 6$ & $671 \pm 24$ & $702 \pm 7$ & $704 \pm 7$ \\
\hline $\mathrm{B}_{2 \mathrm{~g}}\left(\mathrm{~cm}^{-1}\right)$ & $794 \pm 5$ & $768 \pm 6$ & N/A & $777 \pm 12$ & $768 \pm 6$ \\
\hline
\end{tabular}

Raman spectrum from the analysis show three peaks consistent with the previous report for rutile-type $\mathrm{SnO}_{2}$ single crystals [58]. Peaks at around $633\left(\mathrm{~cm}^{-1}\right)$ and $775\left(\mathrm{~cm}^{-1}\right)$ could be assigned to $A_{1 g}$ and $B_{2 g}$, respectively. Due to apparatus limitation, $B_{1 g}$ and $E_{g}$ modes were not resolved from the spectrum. Interestingly, the intense peak around 697 $\mathrm{cm}^{-1}$ which corresponds to the Raman-forbidden mode $\left(\mathrm{A}_{2 \mathrm{u}}\right)$ is also observed. This mode, which is only infrared active, is now seen in the Raman spectrum of $\mathrm{SnO}_{2} \mathrm{NWs}$. This unexpected peak could be associated with a breakdown of the selection rules, or new 
modes called 'volume modes'. This Raman activity is induced by disorder $[56,90,91]$ as the result of sensitivity to local geometric disorientation and to the neighboring disorder (particularly atoms from other sub-lattices or electric defects associated with substitutions or vacancies) [92].

It is also observed that the insertion of impurities into the $\mathrm{SnO}_{2} \mathrm{NW}$ lattice leads to the Raman peak shift ( $\mathrm{A}_{1 \mathrm{~g}}$ mode) toward lower frequency. Compared to $\mathrm{Li}$ doped, n-type $\mathrm{SnO}_{2} \mathrm{NWs}$ have more noticeable red shifts upon the introduction of dopant. This phenomenon is consistent with Mo-doped $\mathrm{SnO}_{2}$ NWs, observed by Yi's group [93] and explained by lattice distortion or lattice symmetry lowering.

\subsubsection{Electrical properties of thick film using n-doped $\mathrm{SnO}_{2} \mathrm{NWs}$}

In this study, silver epoxy was used as the electrical contacting electrode, and plays an important role in forming an Ohmic contact with semi-conductive Sb-doped $\mathrm{SnO}_{2}$ nanomaterial compressed to form a thick film (see chapter 2). Figure 32 also shows I-V characteristics of NWs as function of dopant concentration. The linear dependence between current and voltage from the I-V measurement of each sample shows the Ohmic contact characteristic as expected. This good apparent ohmic contact takes place perhaps due to degenerate doping [94]. As impurity concentration is increased, the as-fabricated sensor resistance significantly decreases and achieves its minimum value at 5\% dopant concentration. However, at higher dopant concentration an increase in the resistance was observed. The resistances of 3\%, 5\% and 10\% doped NW based sensors are $270 \mathrm{k} \Omega, 0.2$ $\mathrm{k} \Omega$ and $2.1 \mathrm{k} \Omega$, respectively. This characteristic is derived from the phase separation 
upon high doping concentration of $\mathrm{Sb}$, which generates an insulating layer $\mathrm{Sb}_{2} \mathrm{O}_{3}$ on the surface of NWs and increases inter-nanowires potential barrier.

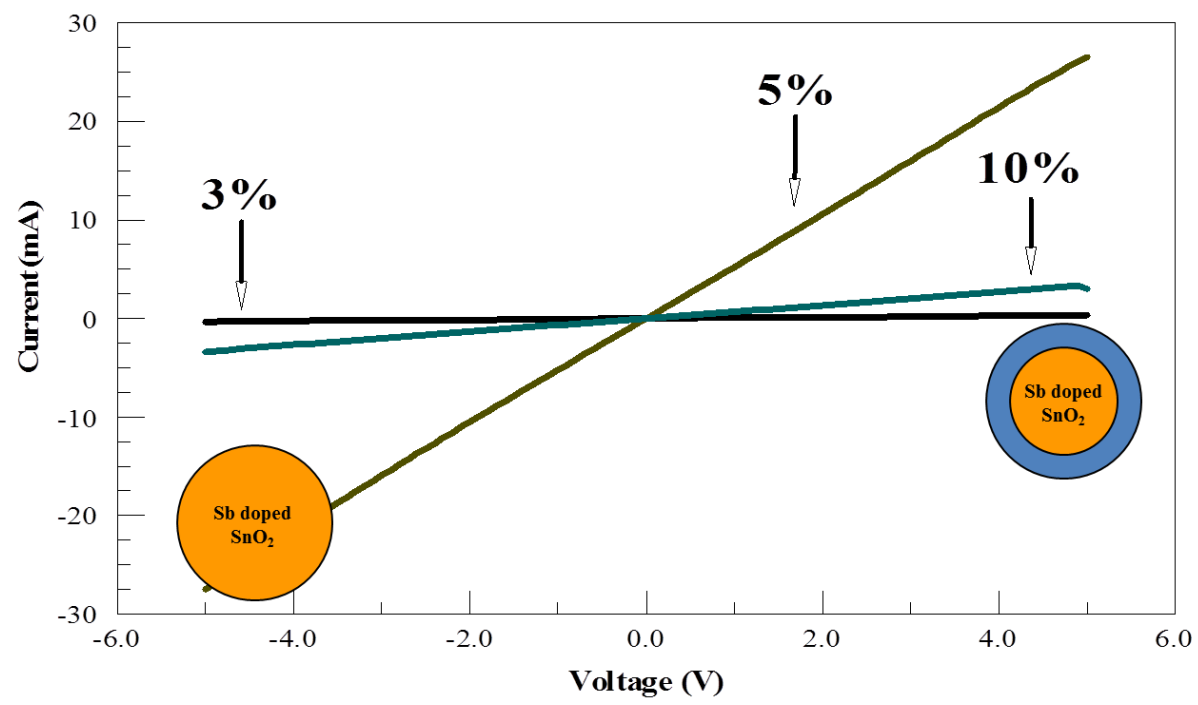

Figure 32: I-V curves of different dopant concentrations of $\mathrm{n}$-doped NW based sensors. The resistances of $3 \%, 5 \%$ and $10 \%$ doped $\mathrm{NW}$ based sensors are $\sim 270 \mathrm{k} \Omega, 0.2 \mathrm{k} \Omega$ and $2.1 \mathrm{k} \Omega$, respectively. 


\subsection{Sensing Application: Chlorine Resistive Gas Sensor Based on n-doped $\mathrm{SnO}_{2} \mathrm{NWs}$}

In this section, the as-fabricated $\mathrm{Sb}$-doped $\mathrm{SnO}_{2} \mathrm{NW}$ based sensor is employed for chlorine gas detection. The sensor performance is investigated through its response, sensitivity and stability toward air-diluted chlorine gas. The study is based on direct current conductivity measurements.

\subsubsection{Sensor response, response time and recovery time}

Typically, the gas response (S) of a resistive sensor is defined as the ratio of the change in resistance of the sensor on exposure to the target gas to the original resistance in air:

$$
S=\frac{R_{\text {gas }}-R_{\text {air }}}{R_{\text {air }}}
$$

Where $\mathrm{R}_{\mathrm{gas}}$ and $\mathrm{R}_{\mathrm{air}}$ are the resistance of the sensor in a target gas medium and in air, respectively

The response time is defined as the time needed for the sensor resistance to change by $90 \%$ of the difference from the maximum value to the minimum after the test gas injection. The recovery time is the time required for the sensor resistance to change by $90 \%$ of the difference from the minimum value to the maximum after releasing the test gas [95]. The response and recovery time at room temperature of $10 \% \mathrm{Sb}$-doped $\mathrm{SnO}_{2} \mathrm{NW}$ based sensor, at six different $\mathrm{Cl}_{2}$ concentrations, are shown in Figure 33 . The response and recovery time at five different temperatures $\left(25^{\circ} \mathrm{C}, 35^{\circ} \mathrm{C}, 45^{\circ} \mathrm{C}, 90^{\circ} \mathrm{C}\right.$, $110^{\circ} \mathrm{C}$ ) are summarized in Figure 34. 
$\mathbf{A}$
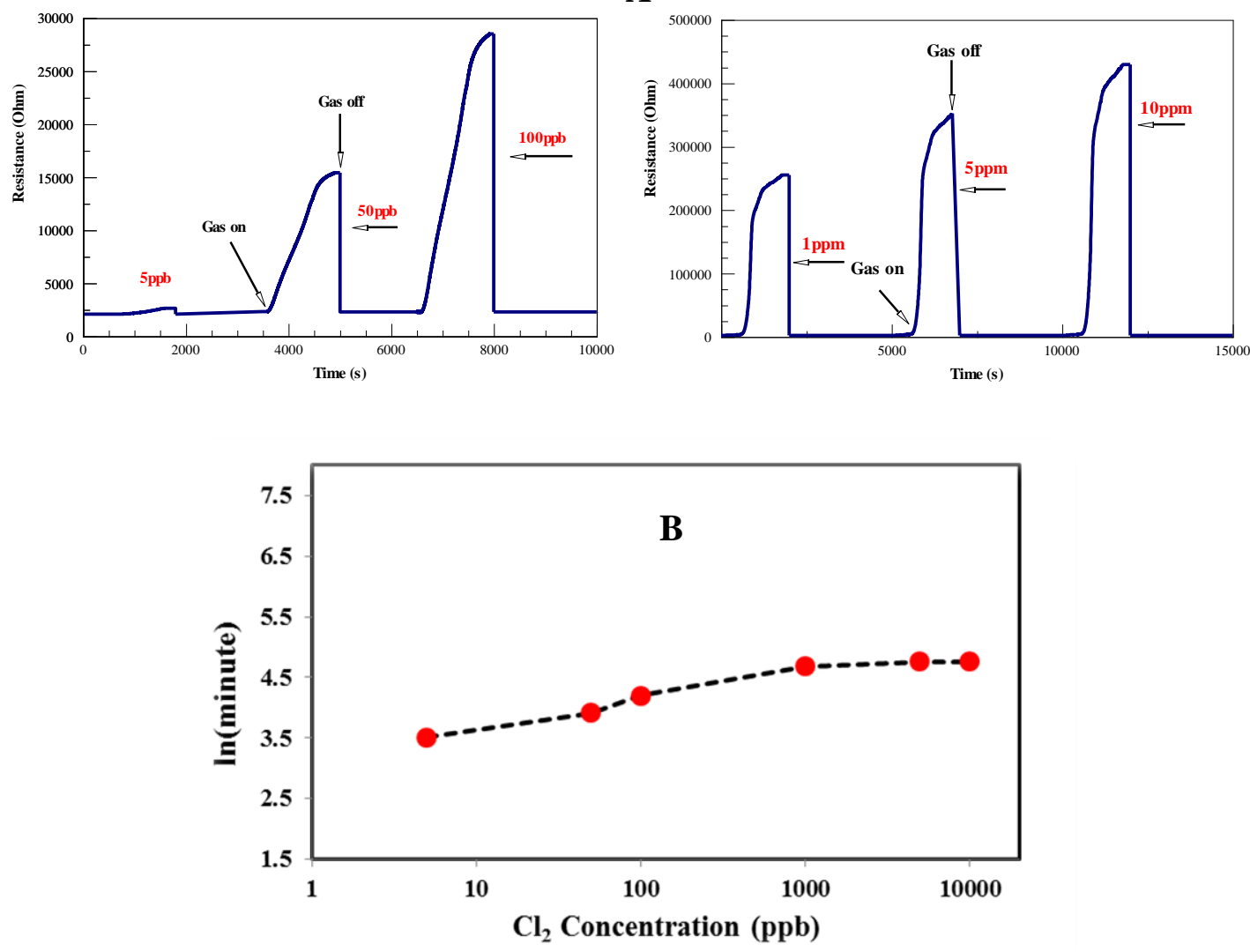

Figure 33: The sensor resistance (A) and recovery time (B) at six different $\mathrm{Cl}_{2}$ concentrations from $5 \mathrm{ppb}$ to $10 \mathrm{ppm}$. The sensor was recovered by introducing fresh air and heating at $45^{\circ} \mathrm{C}$. (Note: Data in Figure $33 \mathrm{~A}$ does not include sensor response during recovery process)

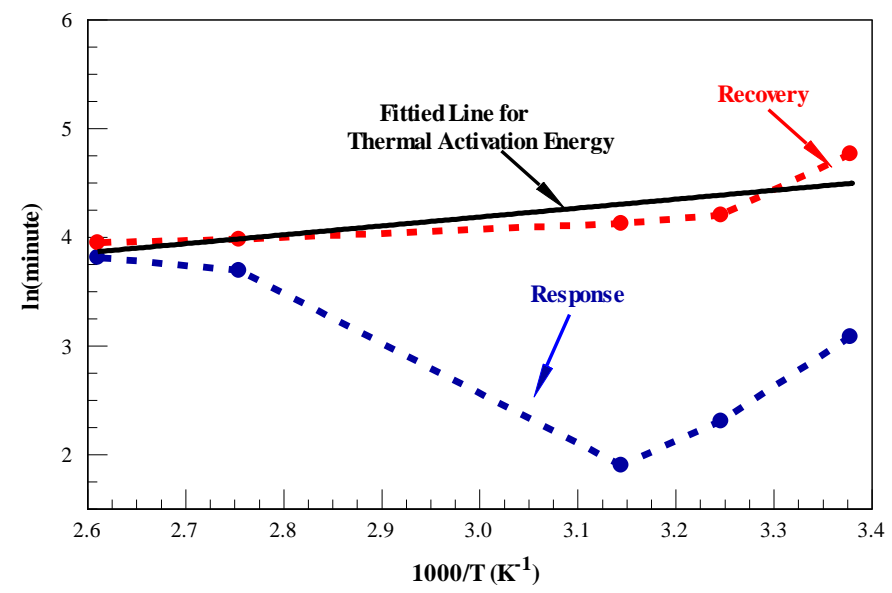

Figure 34: Response and recovery time (minute) as a function of temperature $\left({ }^{\circ} \mathrm{K}\right)$. The slope of a linear fitting from $\ln$ (time) vs $1 / \mathrm{T}$ for recovery gives desorption activation energy of $82 \pm 3 \mathrm{~kJ} / \mathrm{mol}$. 
The response times of the device are short at room temperature but the recovery times appear to be quite long. Specifically, it takes close to 10 minutes for the sensor to respond but about 60 minutes to recover at room temperature after exposure to $1 \mathrm{ppm}$ chlorine. However, it was found that the response kinetics of the device is affected by the kinetics of the gas flow through the testing chamber. As long as the chlorine concentration in the testing chamber still remains in the high ppm range, the response and recovery times cannot be determined accurately. This impact is even more dominated when switching from high to low chlorine concentration detections.

The response and recovery times are temperature dependent as expected from analyte adsorption and desorption processes, respectively. The response time exhibited a minimum around $45^{\circ} \mathrm{C}$ while the recovery time decreased as a function of temperature and followed Arrhenius type behavior (eq. 3.7) [96]. From this, the thermal activation energy from desorption process was determined to be $82 \pm 3 \mathrm{~kJ} /$ mole as shown in Figure 34 above. The value of $E_{a}$ is suggestive of chemisorption of chlorine.

$$
\begin{aligned}
& \text { Rate }=A e^{-\frac{E_{a}}{R T}} \\
& \text { Or } \ln (\text { Rate })=-\frac{E_{a}}{R T}+B
\end{aligned}
$$

Where:

$\mathrm{A}$ and $\mathrm{B}$ are constants; $\mathrm{R}$ is gas constant

$E_{a}$ is activation energy $(\mathrm{KJ} /$ mole $)$

$\mathrm{T}$ is absolute temperature $\left({ }^{\circ} \mathrm{K}\right)$.

Data presented in Figure $33 \mathrm{~A}$ above also show high response $(\mathrm{S} \approx 125$ for 1ppm of $\mathrm{Cl}_{2}$ concentration) and able to detect chlorine at room temperature with relatively fast 
response time compared to current known metal-oxide based sensors for chlorine detection [97-100]. Remarkably, the sensor exhibits a very high sensitivity to chlorine concentration (5 ppbs). This characteristic has been significantly improved from our recent report of a Sb doped- $\mathrm{SnO}_{2}$ NPs based chlorine sensor [34]. Similarly, the undoped $\mathrm{SnO}_{2} \mathrm{NW}$ based sensor shows a relatively poor sensitivity compared to its $\mathrm{Sb}$-doped counterparts reconfirming a potential catalytic effect of $\mathrm{Sb}$, as shown in Figure 17, in improving sensitivity.

\subsubsection{Sensor Sensitivity}

A. Sensor Sensitivity as a function of chlorine concentration

Figure 35 shows the response of the sensor fabricated from $10 \% \mathrm{Sb}$ doped $\mathrm{SnO}_{2}$ NWs to different $\mathrm{Cl}_{2}$ concentrations in air from 5ppb to $10 \mathrm{ppm}$. In this study, the sensor was continuously renewed after every 2 cycles of exposure and recovery. This has minimized the effect of adsorption and desorption processes on the sensor stability, which is discussed in the following section. The response increases non-linearly with increasing chlorine concentrations and tends to get saturated after $1 \mathrm{ppm}$. The continuous flow of analyte can eliminate the effect of adsorption of $\mathrm{Cl}_{2}$ on the chamber wall and contributes to the ability to detect target at very low concentrations. Significantly, chlorine gas can be unprecedentedly detected at ppb level at room temperature. 


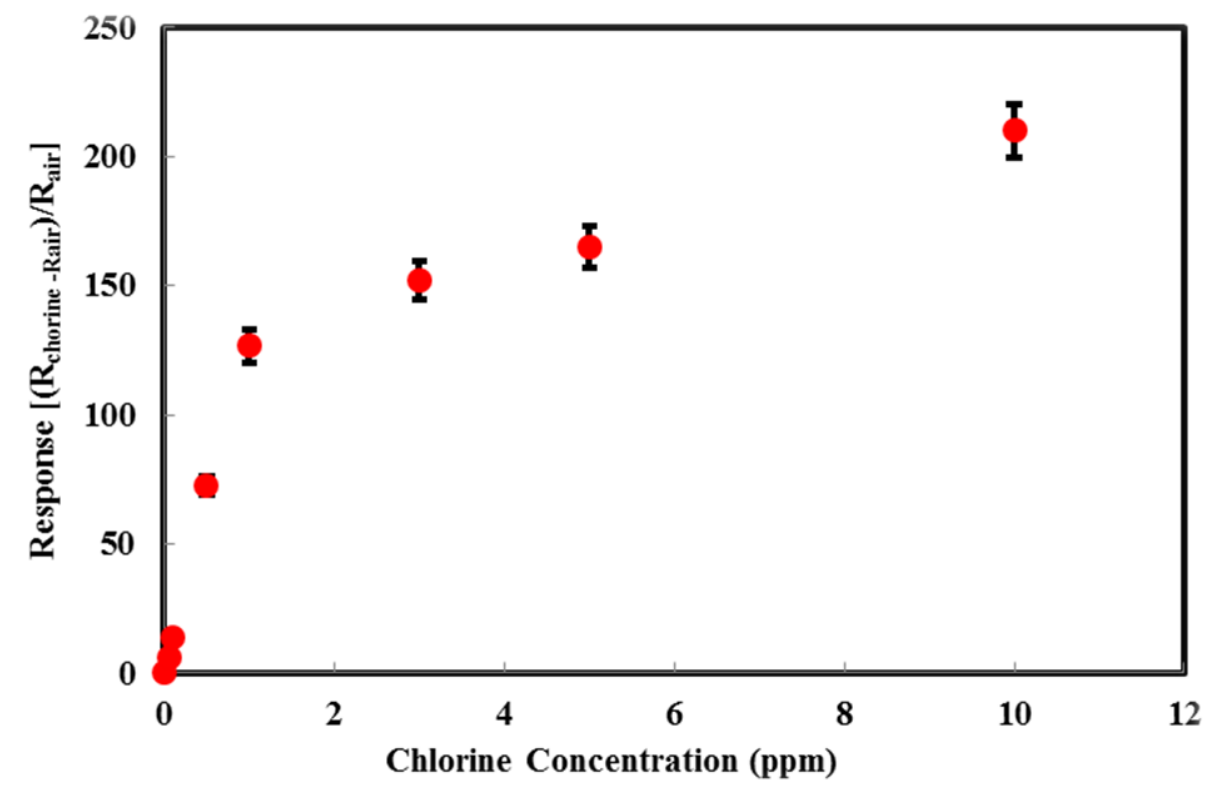

Figure 35: Response as a function of chlorine concentration at room temperature. The sensor response was investigated in the range from $5 \mathrm{ppb}$ to $10 \mathrm{ppm}$ of $\mathrm{Cl}_{2}$ concentrations.

A model used to describe this observed non-linear relationship between concentration and response can be based on a surface-trap-limit model, proposed by Dawson [101]. The equilibrium of a dissociative chemisorption of the gas with sites, S, on the oxide surface is formulated as:

$$
\begin{aligned}
& \mathrm{Cl}_{2}+2 S \leftrightharpoons C l+C l \\
& \mathrm{O}_{2}+2 S \leftrightharpoons O+O
\end{aligned}
$$

These reactions are formulated as a competitive adsorption of chlorine and oxygen:

$$
\begin{aligned}
& \mathrm{O}+1 / 2 \mathrm{Cl}_{2} \leftrightharpoons \mathrm{Cl}+1 / 2 \mathrm{O}_{2} \\
& \frac{\theta_{C l} P_{O_{2}}^{\frac{1}{2}}}{\theta_{O} P_{C l_{2}}^{\frac{1}{2}}}=\frac{\theta_{C l}}{\theta_{O}} \gamma^{-\frac{1}{2}}=K_{a d s}
\end{aligned}
$$

Where $\theta$ is the fractional coverage over the surface, $P$ is the partial pressure and $\gamma=\frac{P_{C l_{2}}}{P_{O_{2}}}$ 
In the chemisorption process, the uncharged adsorbed species on the surface of metal oxide acts as a surface electron trap. The equilibriums can be represented as:

$$
\begin{aligned}
& n^{\prime}+O \leftrightharpoons O^{-} \\
& n^{\prime}+C l \leftrightharpoons C l^{-} \\
& \frac{\theta_{C l^{-}}}{n^{\prime} \theta_{C l}}=K_{C l} \\
& \frac{\theta_{O^{-}}}{n^{\prime} \theta_{O}}=K_{O}
\end{aligned}
$$

If it is assumed that the uncharged and charged species are the major and minor surface coverage species, respectively, then at equilibrium:

$$
\begin{aligned}
& \theta_{O}+\theta_{C l} \approx 1 \\
& \theta_{O^{-}}+\theta_{C l^{-}}=\mathrm{q}
\end{aligned}
$$

Where $\mathrm{q}$ is a constant with $\mathrm{q}<<1$. From eq. 3.8, we have:

$$
\left(\frac{q}{K_{O}}\right)\left(\frac{1}{n^{\prime}}\right)=\frac{1+\frac{K_{C l} K_{a d s}}{K_{O}} \gamma^{1 / 2}}{1+K_{a d s} \gamma^{1 / 2}}
$$

Since the left-hand side of eq. 3.9 is proportional to the resistance $\mathrm{R}$ of the sensor, by considering the limit of low and high concentration of chlorine, we have:

$$
\frac{R}{R_{0}}=\frac{1+\frac{K_{C l} K_{a d s}}{K_{O}} \gamma^{1 / 2}}{1+K_{a d s} \gamma^{1 / 2}}=\frac{1+K_{r e l} K_{a d s} \gamma^{1 / 2}}{1+K_{a d s} \gamma^{1 / 2}}
$$

(eq. 3.10)

Where $\frac{K_{C l}}{K_{O}}=K_{\text {rel }}$

If $R_{\infty}$ and $\mathrm{R}_{0}$ denote the resistance response of the sensor in the limit of high and low concentration of chlorine, then we will have $K_{a d s} \gamma^{1 / 2} \gg 1$ : 


$$
\frac{R_{\infty}}{R_{0}}=\frac{K_{C l}}{K_{O}}=K_{r e l} \text { and } K_{r e l} \gg 1
$$

The equation can be written as:

$$
\frac{R-R_{0}}{R_{0}}=\frac{\left(K_{r e l}-1\right) K_{a d s} \gamma^{1 / 2}}{1+K_{a d s} \gamma^{1 / 2}} \approx \frac{K_{r e l} K_{a d s} \gamma^{1 / 2}}{1+K_{a d s} \gamma^{1 / 2}}
$$

The resulting expression bears similarity to the Langmuir model. The curve fitting of sensor response as a function of $\gamma^{1 / 2}$ based on eq. 3.11 is shown in Figure 36

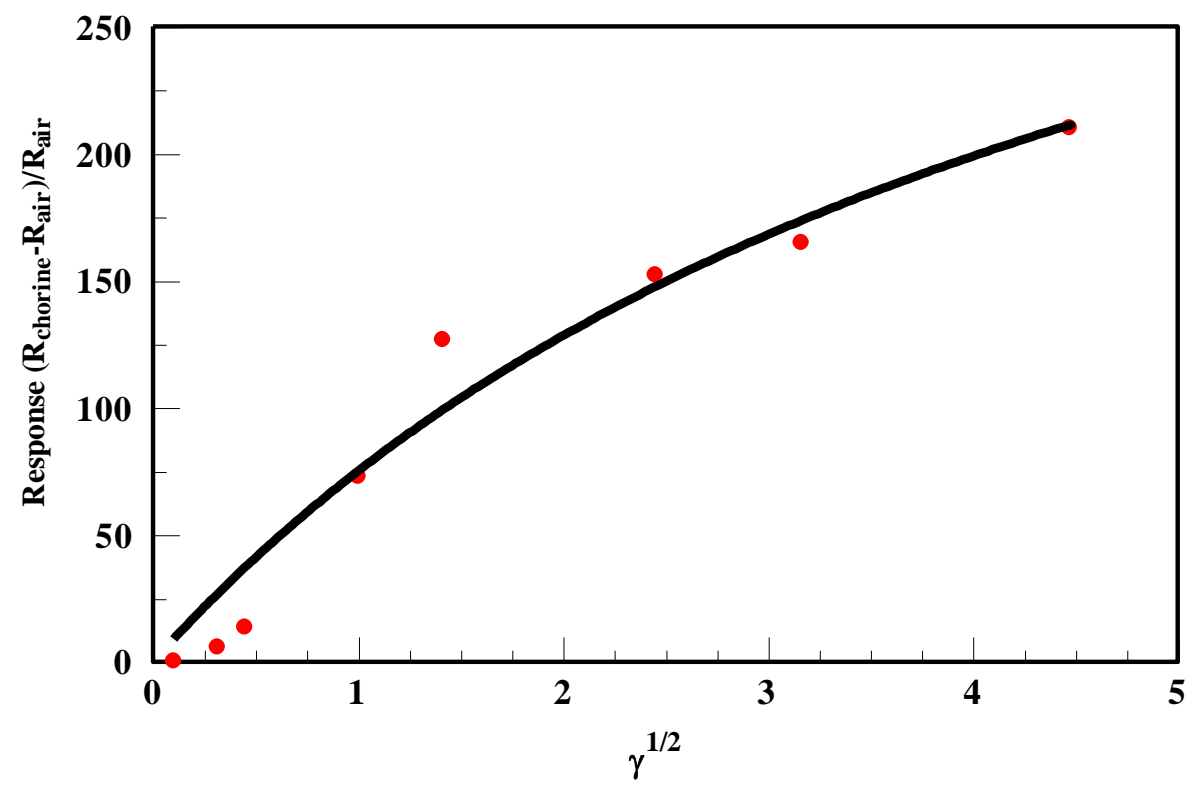

Figure 36: Response of the sensor as a function of $\gamma^{1 / 2}$. Red points and black solid curve are experimental and fitting data, respectively. $\mathrm{K}_{\mathrm{rel}}$ and $\mathrm{K}_{\mathrm{ads}}$ determined from the curve fitting were $450 \pm 50$ and $0.2 \pm 0.1$, respectively.

$$
\text { Since } \frac{K_{C l}}{K_{O}}=K_{r e l}, K_{r e l} \gg 1 \text { has indicated that the energy of the state represented }
$$
by $\mathrm{Cl}_{\mathrm{ads}}$ is much lower than the energy of the state of $\mathrm{O}_{\mathrm{ads}}$ as anticipated from the redox levels for oxygen and chlorine (Figure 17). Upon doping $\mathrm{SnO}_{2}$ by $\mathrm{Sb}$, the chemisorption activation energy of chlorine compared to oxygen on $\mathrm{Sb}$-doped $\mathrm{SnO}_{2}$ is significantly reduced. Furthermore, since $\mathrm{K}_{\mathrm{rel}}$ is the ratio of the resistance of sensor in the high and low 
concentration of chlorine, the value of $\mathrm{K}_{\mathrm{rel}}$ also provides the estimation of the maximum sensor response, which is around $450 \pm 50$.

\section{B. Sensor Sensitivity as a function of doping concentration:}

It is well-known that a large amount of $\mathrm{Sb}$ can be incorporated into a $\mathrm{SnO}_{2}$ lattice to enhance its conductivity [102] leading to a change of sensor performance. The response of the sensor as a function of dopant concentration $(\mathrm{Sb})$ was investigated to determine the optimal dopant concentration for highly sensitive devices. The data in Figure 37 shows that the response of the sensor, and thus sensitivity, is optimal at the doping level of the synthetic condition of about $10 \%$ (mole Sb/mole Sn). At higher doping levels, both conductivity and response decrease.

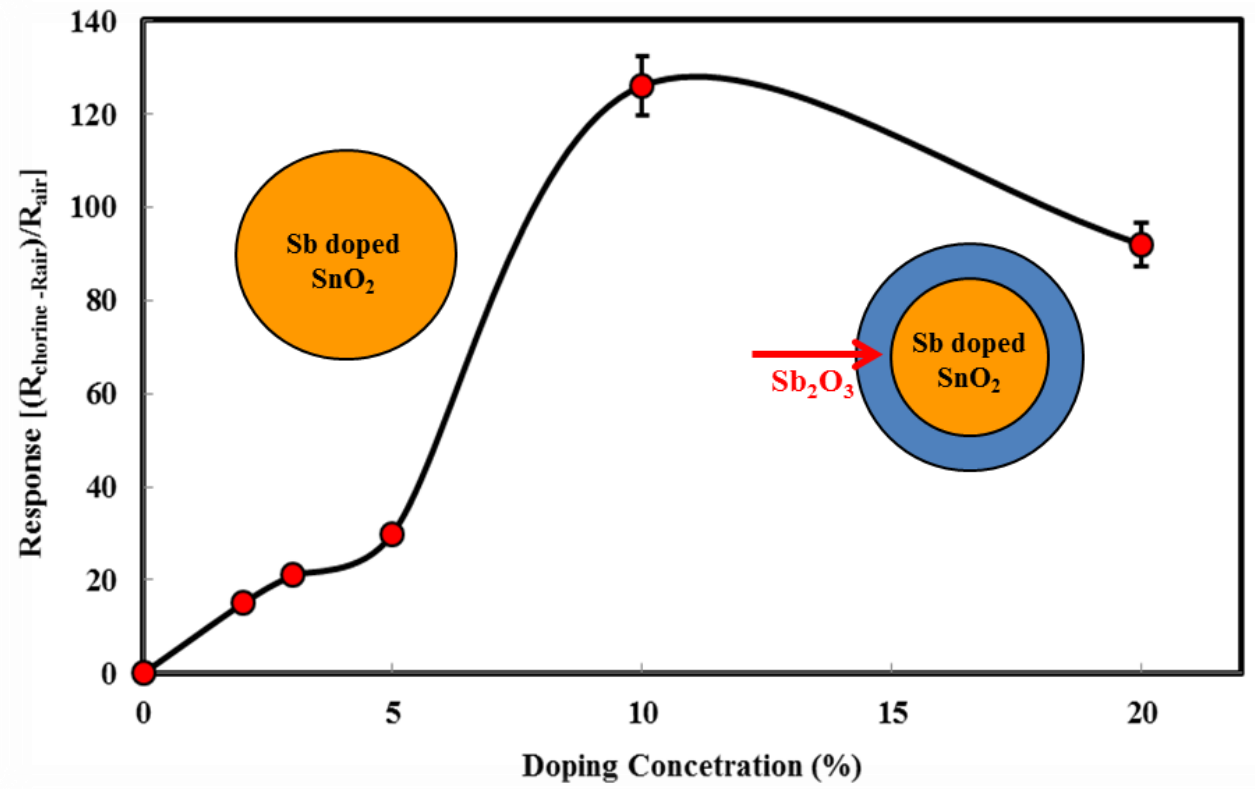

Figure 37: Response of the sensor as a function of doping concentration ( $\mathrm{Sb} / \mathrm{Sn}$ mole ratio). The sensor response is optimal at $10 \%$ doping. At very high doping concentration, sensor response decrease perhaps due to the formation of $\mathrm{Sb}_{2} \mathrm{O}_{3}$ on the surface of NWs.

It has been established that the incorporation of high concentration of $\mathrm{Sb}$ into $\mathrm{SnO}_{2}$, dopant atoms are concentrated near the surface of the nanostructures and induces 
surface segregation of $\mathrm{Sb}$ to $\mathrm{Sb}^{3+}$ and $\mathrm{Sb}^{5+}$ [103-105]. These surface segregated $\mathrm{Sb}$ atoms may form oxygen vacancy complexes at the surface and may be important active sites for adsorption of electron-withdrawing gas molecules [106]. Therefore, the mechanism for the adsorption of chlorine of the surface of Sb-doped $\mathrm{SnO}_{2} \mathrm{NWs}$ at room temperature can be proposed as [34]:

$$
\begin{aligned}
& \mathrm{Cl}_{2} \rightarrow 2 \mathrm{Cl} . \\
& \mathrm{Cl} \cdot+e^{-} \underset{S b^{3+}, \mathrm{Sb}^{5+}}{\longrightarrow} C l^{\prime}
\end{aligned}
$$

Since the response of the sensor is proportional to concentration of segregated $\mathrm{Sb}$ atoms on the surface of NWs, the response increases along with increases of the doping concentration. However, at very high doping concentration, formation of insulating oxide $\mathrm{Sb}_{2} \mathrm{O}_{3}$, increases the barrier for abstraction of an electron from semiconductor nanowires, and leads to the decrease of conductivity as well as response of the sensor as showed in Figure 37.

\subsubsection{Sensor Stability and Understanding the Sensing Mechanism}

A. More specific model of sensing mechanism

The chlorine gas sensing response of metal oxide sensor is based on the chemisorption model. In particular, chlorine is presumed to react with metal oxides to form chlorides or displace lattice oxygen at the interface, or possibly adsorb onto surface oxygen vacancies. The chemisorption chlorine involves mainly four kinds of adsorption behavior in the reactions below [101, 107, 108]:

$$
\begin{aligned}
& \mathrm{Cl}_{2}+2 \mathrm{O}_{2 \text { surf }}^{\prime \prime} \leftrightharpoons 2 \mathrm{Cl}_{\text {surf }}^{\prime}+2 \mathrm{O}_{2}(g)+2 e^{\prime} \\
& \mathrm{Cl}_{2}+2 \mathrm{O}_{O}^{x} \leftrightharpoons 2 C l_{\text {osurf }}+\mathrm{O}_{2}(g)+2 e^{\prime}
\end{aligned}
$$




$$
\begin{aligned}
& C l_{2}+2 e^{\prime} \leftrightharpoons 2 C l_{\text {surf }}^{\prime} \\
& C l_{2}+2 V_{O}^{\prime}+2 e^{\prime} \leftrightharpoons 2 C l_{O}
\end{aligned}
$$

Where subscripts, surf and o, mean the species adsorbed on the surface and the species occupying lattice oxygen sites, respectively. $V_{O}^{\prime \prime}$ is an oxygen vacancy bearing double positive charge, and $\mathrm{x}$ denotes charge neutrality and e represents the conduction electron. Positive charge and negative charge are marked by a point (e.g. A.) and a hyphen (e.g. A'), respectively. In the mechanism (I) and (II), chlorine substitutes for adsorbed oxygen and lattice oxygen to form $\mathrm{Cl}^{\prime}$ and donates electrons to the conduction band of the semiconductor, which should result in a decrease of resistance, and thus inconsistent with the observed results in this study. On the other hand, in the mechanism (III), chlorine is adsorbed on the surface and draws electrons from the semiconductor to form $\mathrm{Cl}^{\prime}$, leading to an increase of resistance. Similarly for the mechanism (IV), absorbed chlorine occupies the oxygen vacancy along with drawing electrons to become chlorine ions. This mechanism also results in the increase of resistance.

Intuitively, both of these mechanisms (III) and (IV) are probable upon the exposure of $\mathrm{Cl}_{2}$ gas, corresponding to the reaction of $\mathrm{Cl}_{2}$ on the surface and inside the lattice of $\mathrm{Sb}$-doped $\mathrm{SnO}_{2} \mathrm{NWs}$. This model was already incorporated at the beginning of section 3.4.2. With the mechanism (III), creation of the chloride ion formed on the oxide surface would lead to a depletion layer by repelling majority carrier away from the surface as illustrated in Figure 38. A depletion layer as well as inter-nanowire barrier that hinders electron transport hence increases the sensor resistance. 


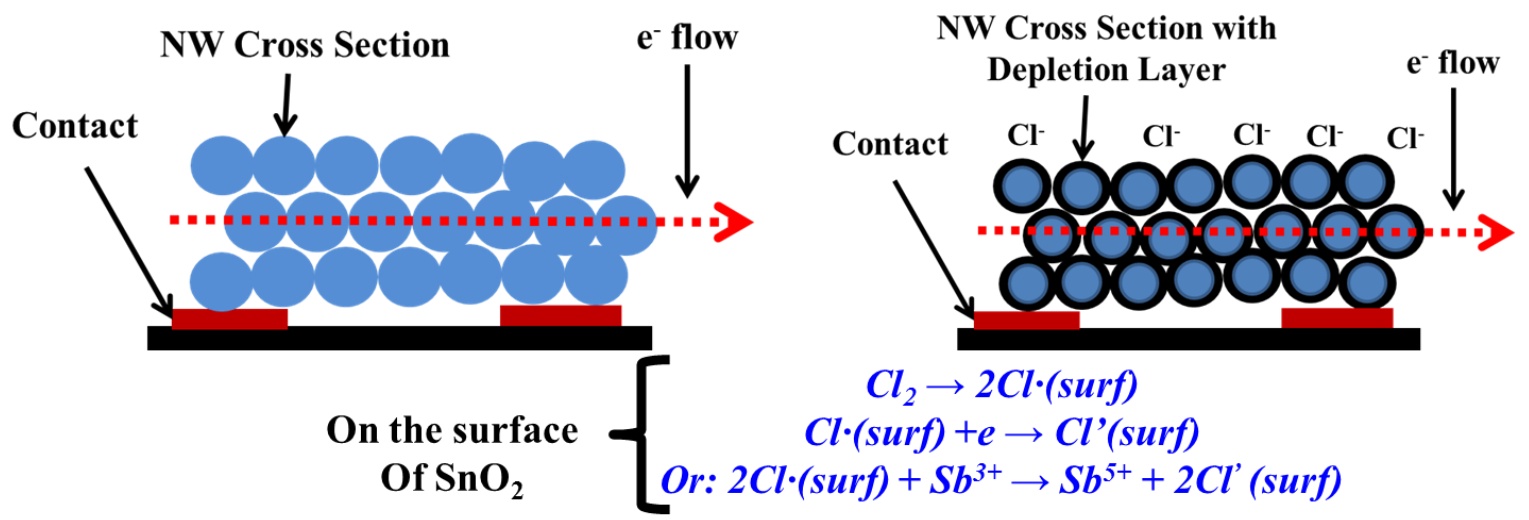

Figure 38: Schematic diagram of the porous sensing layer composed from nanowires (each circle illustrates $\mathrm{NW}$ cross section). There is no depletion layer in the absence of $\mathrm{Cl}_{2}$. Upon exposure to $\mathrm{Cl}_{2}$, the depletion layer is generated as a result of $\mathrm{Cl}^{\prime}$ formation on the surface of NWs.

To investigate the effect of mechanism (IV) on the response of the sensor, the experiments based on multiple times of repeated exposure and refresh cycles on the same sensor were carried out. Data below show the response of the sensor upon the exposure to $1 \mathrm{ppm} \mathrm{Cl}_{2}$ gas, the senor recovery was achieved by a flow of air combining with heating the sensor at $45^{\circ} \mathrm{C}$.

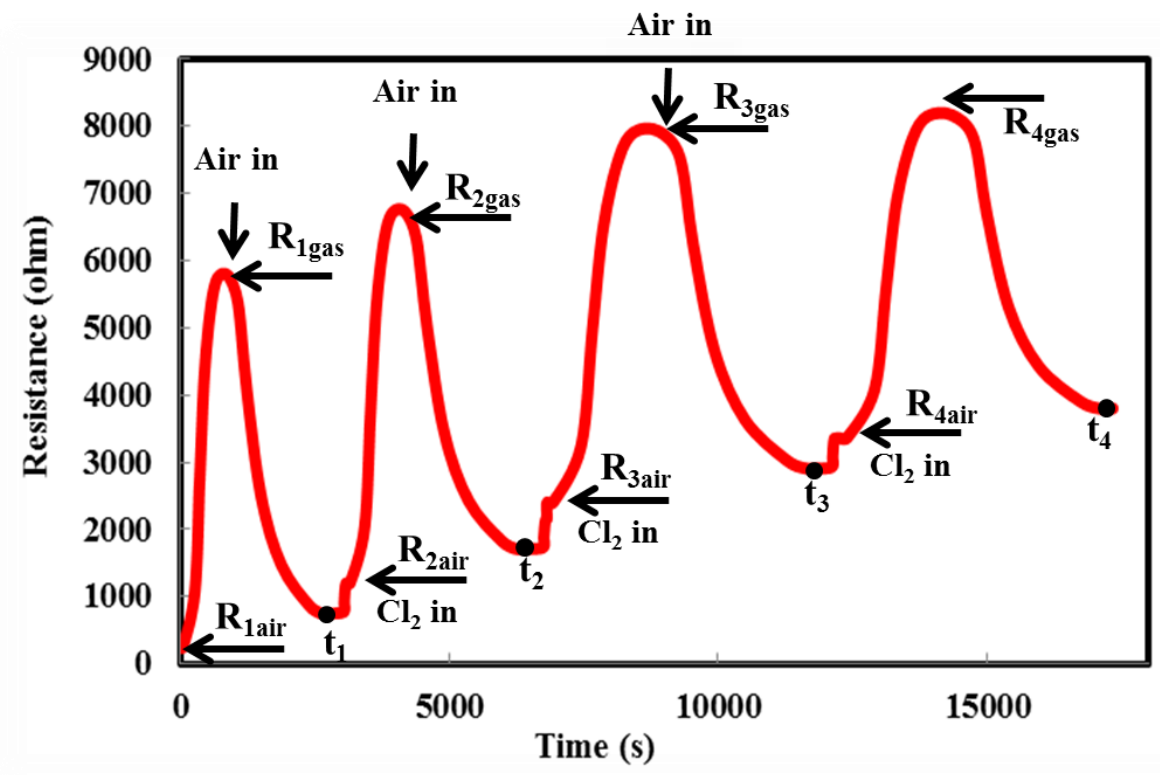

Figure 39: Response of the sensor from 4 continuous cycles of exposure and recovery. Sensor resistance (R) and cycle time (t) were recorded to investigate the effect of mechanism (IV). 


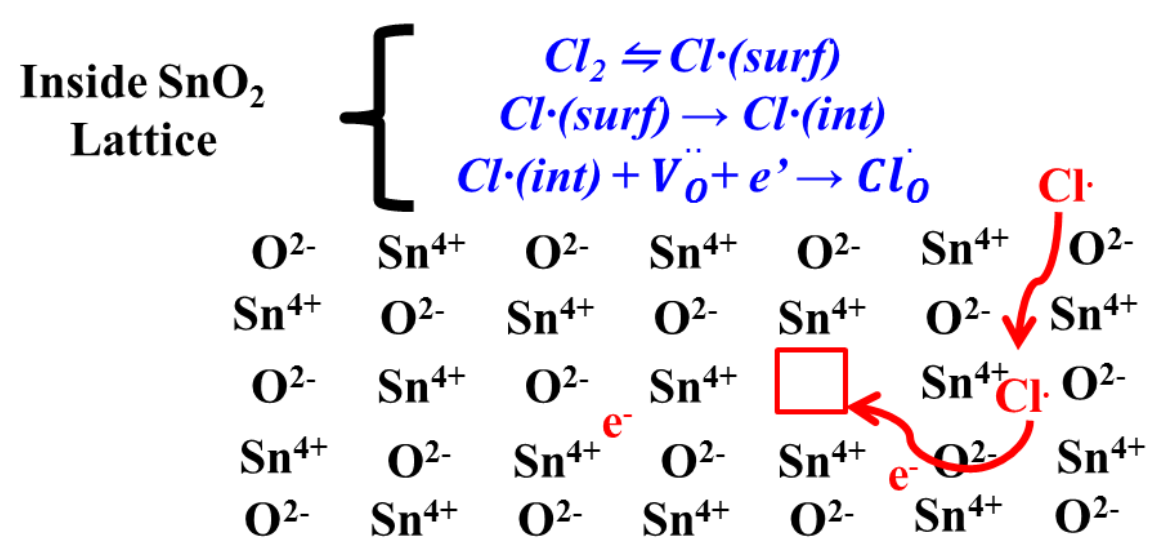

Figure 40: Illustration of mechanism (IV). The square inside the $\mathrm{SnO}_{2}$ lattice represents oxygen vacancy, which is occupied by diffused $\mathrm{Cl}$. This process leads to electron deficiency inside $\mathrm{SnO}_{2}$, causing sensor resistance baseline drifting.

It can be seen that the baseline resistance drifts with the increase of initial sensor's resistance $\left(\mathrm{R}_{\text {air }}\right)$ after every cycle of exposure and recovery. The increase of $R_{\text {air }}$ has led to the sensor response $(S)$, which is defined as $\left(R_{\text {gas }}-R_{a i r}\right) / R_{a i r}$, to decrease over time. Explanation for this characteristic can be based on mechanism (IV) and illustrated in Figure 40. Particularly, chlorine gas molecules are absorbed on the surface of doped $\mathrm{SnO}_{2} \mathrm{NWs}$ and dissociate into two chlorine atoms, which subsequently diffuse into to the bulk $\mathrm{SnO}_{2} \mathrm{NW}$ lattice. These chlorine atoms replace oxygen vacancies along with drawing electrons from the conduction band of $\mathrm{Sb}$-doped $\mathrm{SnO}_{2}$ to become chloride ions occupying oxygen vacancies. This mechanism contributes to the drifting of the sensor resistance baseline after every cycle of exposure and recovery.

The drift of baseline resistance as a function of chlorine exposure time can be modeled as first order kinetic process described by follows equation:

$$
R=R_{0}\left(1-e^{-k t}\right)
$$


Where $\mathrm{R}_{0}$ is the maximum resistance, resulting from complete saturation of oxygen vacancies by chloride ions

The plot in Figure 41 shows resistance drifting as a function of time. From this, the maximum drifting resistance value $R_{0}(14000 \pm 500 \Omega)$ was achieved by curve fitting based on eq. 3.12 .

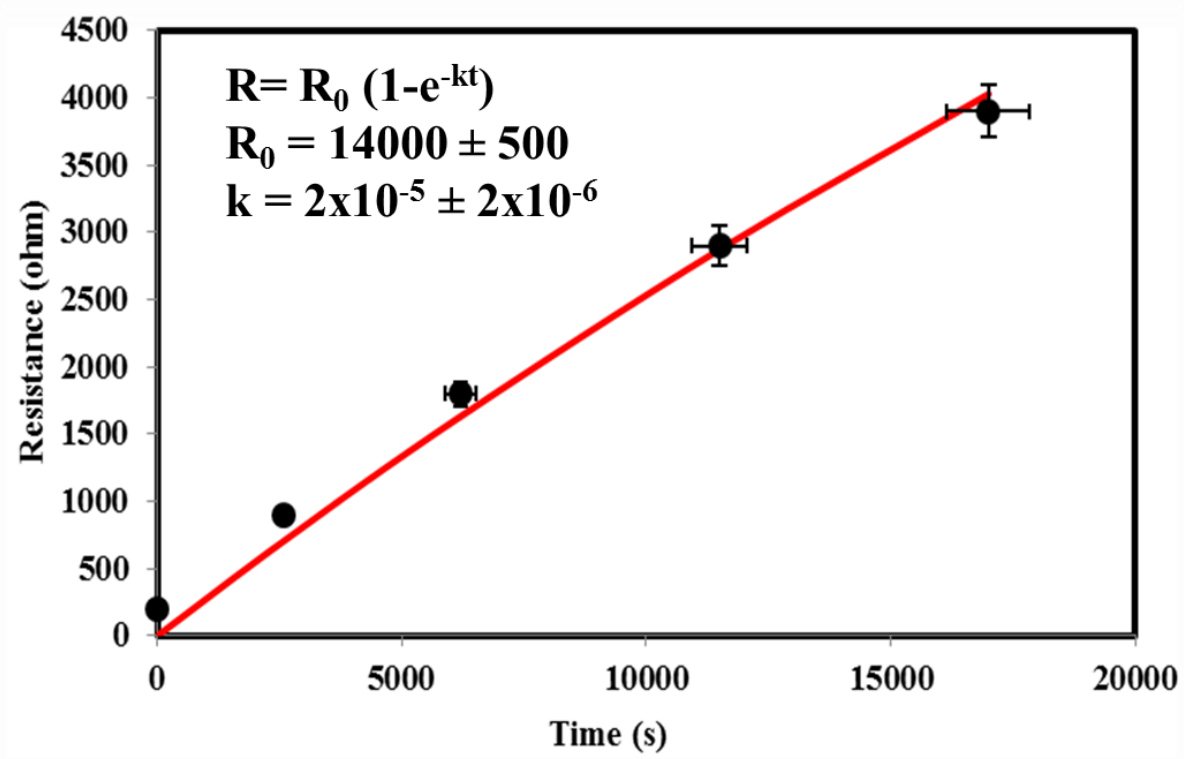

Figure 41: Sensor resistance baseline as a function of cycle time. 5 black data points in the graph correspond to $t_{0}, t_{1}, t_{2}, t_{3}, t_{4}$. Solid red curve shows the fitting following eq. 3.12.

In order to further confirm the effect of chloride ions occupying oxygen vacancies, which led to the shift of the sensor resistance baseline, we have carried out an experiment by storing the sensor in very high concentration of chlorine gas (99\%). After 8 hours of storage, maximum sensor resistance was achieved to be $\sim 59.5 \mathrm{k} \Omega$. Following this, the sensor was refreshed with air over night at room temperature. At this point, sensor resistance was measured to be around $8 \mathrm{k} \Omega \mathrm{s}$. The sensor performance was then investigated (data presented in Figure 42) by exposing to $1 \mathrm{ppm}$ chlorine concentration 
and recovered by a flow of dried air along with heating at $100^{\circ} \mathrm{C}$ in 8 hours. The stabilized sensor resistance after cooling down to room temperature was measured to be $5.1 \mathrm{k} \Omega \mathrm{s}$.

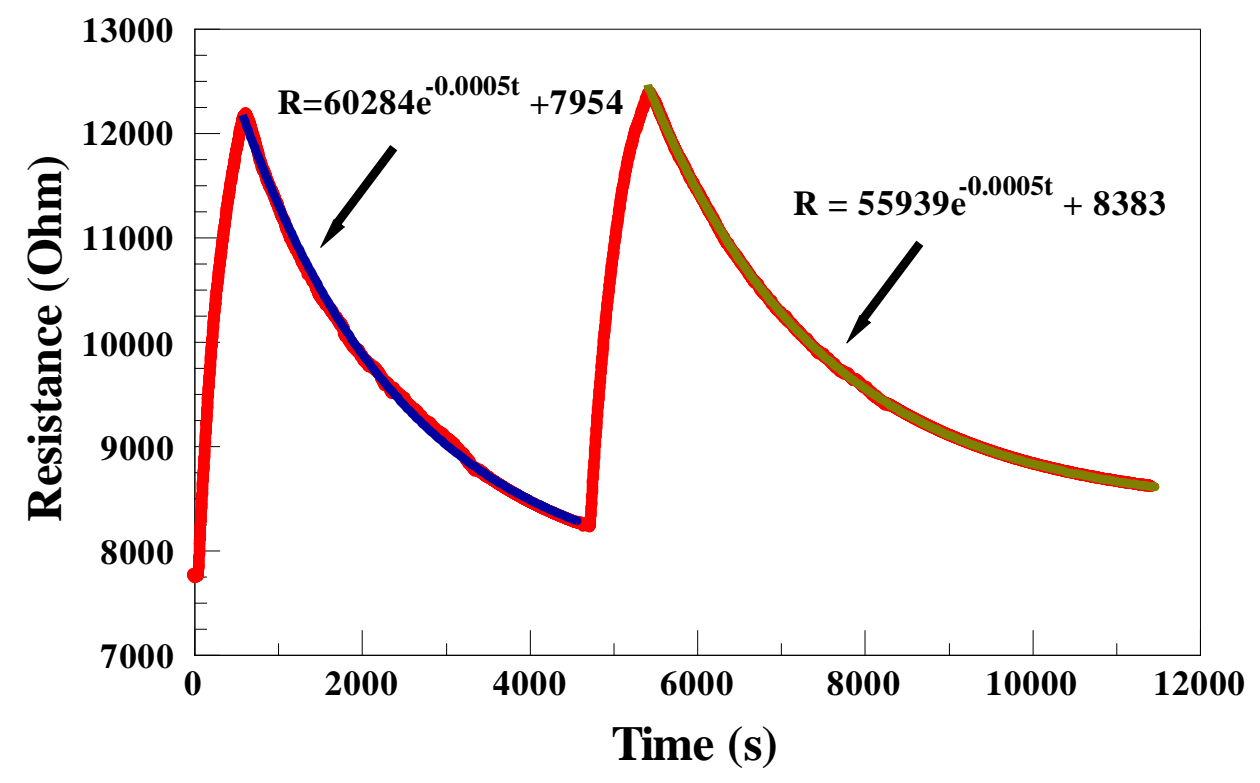

Figure 42: Sensor response curves after storing in high $\mathrm{Cl}_{2}$ concentration, the sensor performance was investigated by exposing to $1 \mathrm{ppm} \mathrm{Cl} \mathrm{Cl}_{2}$ gas. There was not much shift of sensor resistance baseline in this case.

As can be observed from Figure 42, when the sensor was stored under high chlorine concentration, mechanism(III) and (IV) has taken place, and oxygen vacancies were completely replaced by chloride ions, leading to maximum sensor resistance $(59.5$ $\mathrm{k} \Omega$ ). After refreshing with air for next the performance investigation, the resistance baseline drifting was significantly reduced. The resistance after recovery stabilized at almost the same level as before the exposure $(\sim 8 \mathrm{k} \Omega \mathrm{s})$. At this point, the exposure and recovery were simply based on the adsorption and desorption processes (mechanism III), which took place only on the surface of $\mathrm{SnO}_{2} \mathrm{NWs}$, leading to a better sensor performance characteristic. When the sensor was refreshed with air under high 
temperature $100^{\circ} \mathrm{C}$ in 8 hours, the reverse mechanism (IV) took place for oxygen vacancy recovery, leading to the decrease of sensor resistance.

\section{B. Sensor response kinetics}

For every cycle of the exposure and recovery process, the sensor response was investigated in terms of a simple successive reaction kinetic model. The kinetic response during the exposure process can be assumed to follow two steps. Chlorine gas molecules firstly approach the surface of $\mathrm{SnO}_{2}$ and dissociate into chlorine atoms, which subsequently extract electrons from $\mathrm{SnO}_{2}$ conduction band. This can be presented in equation below:

$$
C l_{2(\text { surf })} \stackrel{k_{1}}{\rightarrow} 2 C l \cdot+2 e \stackrel{k_{2}}{\rightarrow} 2 C l_{\text {surf }}^{\prime} 1
$$

Where $\mathrm{k}_{1}$ and $\mathrm{k}_{2}$ are dissociation and electron extraction kinetic constants of chlorine atoms on the surface of $\mathrm{SnO}_{2}$, respectively.

Therefore, the change of sensor resistance as a function of time can be written as:

$$
R=R_{0}\left(1-\frac{k_{1}}{k_{1}-k_{2}}\left(e^{-k_{1} t}-e^{-k_{2} t}\right)-e^{-k_{1} t}\right)+\mathrm{C}
$$

Where $\mathrm{R}_{0}$ and $\mathrm{C}$ are the maximum sensor resistance and the average value of baseline resistance during a cycle, respectively

The fitting using eq. 3.12 for the first exposure cycle is illustrated in Figure 43.

\footnotetext{
${ }^{1}$ The breakdown of $\mathrm{Cl}-\mathrm{Cl}$ bond may proceed through 2 steps:

$\mathrm{Cl}_{2}+e \rightarrow \mathrm{Cl}_{2}^{\prime} \rightarrow \mathrm{Cl}^{\prime}+\mathrm{Cl}$.

$\mathrm{Cl} \cdot+e \rightarrow C l^{\prime}$

However, the detection of $\mathrm{Cl} \cdot$ was not pursued.
} 


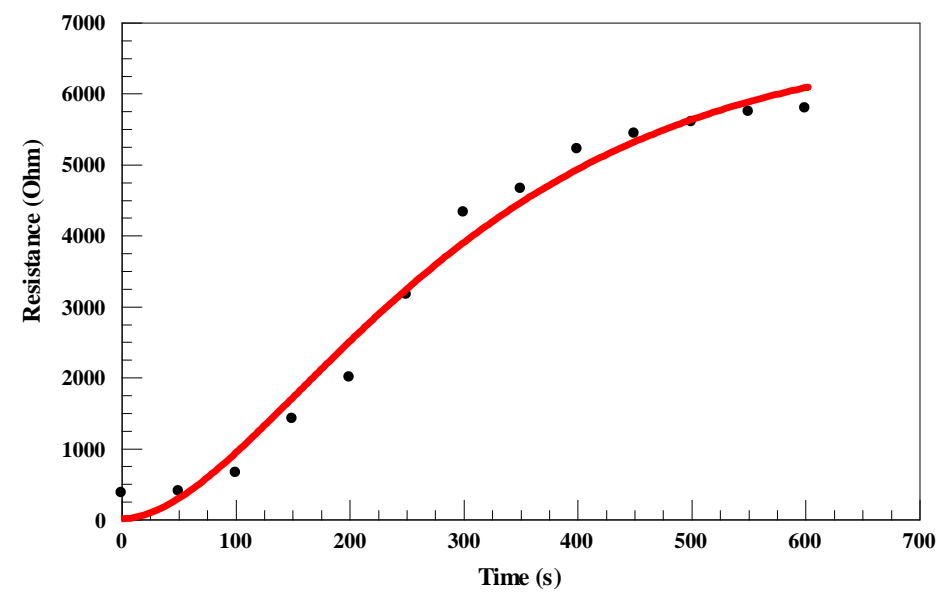

Figure 43: An example of fitting sensor response resistance as a function of time following (eq. 3.13)

Table 7: Kinetic analysis of response curves during the exposure process.

\begin{tabular}{|c|c|c|c|c|}
\hline & $1^{\text {st }}$ cycle & $2^{\text {nd }}$ cycle & $3^{\text {rd }}$ cycle & $4^{\text {th }}$ cycle \\
\hline $\mathrm{k}_{1}\left(\mathrm{~s}^{-1}\right)\left(\times 10^{3}\right)$ & $6 \pm 2$ & $5.3 \pm 0.5$ & $4.0 \pm 0.3$ & $4.0 \pm 0.5$ \\
\hline $\mathrm{k}_{2}\left(\mathrm{~s}^{-1}\right)\left(\mathrm{x} 10^{3}\right)$ & $6 \pm 2$ & $5.3 \pm 0.5$ & $4.0 \pm 0.3$ & $2.5 \pm 0.3$ \\
\hline $\mathrm{R}_{0}(\mathrm{ohm})\left(\times 10^{-3}\right)$ & $7.0 \pm 2$ & $7.0 \pm 1$ & $6.0 \pm 1$ & $6.0 \pm 1$ \\
\hline $\mathrm{C}(\mathrm{ohm})\left(\mathrm{x} 10^{2}\right)$ & $2.0 \pm 0.5$ & $9.0 \pm 0.9$ & $2.7 \pm 0.1$ & $3.3 \pm 0.1$ \\
\hline
\end{tabular}

The results of kinetic constants of chlorine dissociation and electron abstraction on the surface of Sb-doped $\mathrm{SnO}_{2}$ are summarized in Table 7. As can be seen, there is not much change in the dissociation rate constant $\left(\mathrm{k}_{1}\right)$ with successive cycles of adsorption and desorption. The difference in rate constant $\left(\mathrm{k}_{2}\right)$ is more obvious between the first cycles corresponding to "fresh surface" and $4^{\text {th }}$ cycle corresponding to "unclean surface". The almost two times decrease in the rate constant suggests that the oxygen vacancies replaced by chlorine slow down the electron transfer rate from the conduction band of $\mathrm{SnO}_{2}$ to the adsorbed chlorine atom.

When the sensor is recovered, reverse reactions including surface desorption and oxygen vacancy recovery are expected. Trapped electrons during the chorine absorption process could be released and donated back to the conduction band of Sb-doped $\mathrm{SnO}_{2}$ by 
reaction between chlorides and oxygen in air, as represented by eq. 3.14 and eq.3.15. This would cause the increase of electron concentration, and thus conductivity. However, complete refresh is just only achieved at elevated temperatures upon the reversed reaction of mechanism (IV) or the recovery of oxygen vacancies. Because the diffusion of chlorine out of defect is absolutely negligible at the temperature used in this experiment [109], chloride species still remain on the near surface and inside the $\mathrm{SnO}_{2}$ lattice, leading to the depletion of charge carrier concentration, causing drifting of sensor resistance baseline as analyzed above.

Understanding of kinetic mechanism during this recovery process was based on the continuous cycles of exposure and recovery data. Reactions, causing the decrease of sensor resistance, between chloride ions absorbed on the surface of $\mathrm{Sb}$ doped $\mathrm{SnO}_{2} \mathrm{NWs}$ as well as with oxygen gas in air can be represented as:

$$
\begin{aligned}
& \mathrm{Cl}^{-}+\mathrm{O}_{2} \stackrel{k_{1}}{\rightarrow} \mathrm{ClO}_{2}+e^{-} \\
& (\text {eq. 3.14) } \\
& C l^{-}+C l^{-} \stackrel{k_{2}}{\rightarrow} C l_{2}+2 e^{-} \\
& \frac{(\text { eq. 3.15) }}{d t}=-k_{1}\left[C l^{-}\right]-k_{2}\left[C l^{-}\right]^{2} \\
& -d t=\frac{d\left[C l^{-}\right]}{\left[C l^{-}\right]\left(k_{1}+k_{2}\left[C l^{-}\right]\right)} \\
& {\left[C l^{-}\right]=\frac{k_{1} e^{-k_{1} t+c}}{1-k_{2} e^{-k_{1} t+c}}} \\
& R=R_{0} \frac{k_{1} e^{-k_{1} t+c}}{1-k_{2} e^{-k_{1} t+c}}
\end{aligned}
$$

Where $\mathrm{C}$ is a constant. $\mathrm{k}_{1}$ and $\mathrm{k}_{2}$ are the reaction kinetic constants. 
These kinetic constants (eq. 3.14 and eq. 3.15) can be evaluated from fitting of the sensor resistance change as a function of time followed eq. 3.16. An example of this fitting is illustrated in Figure 44 below:

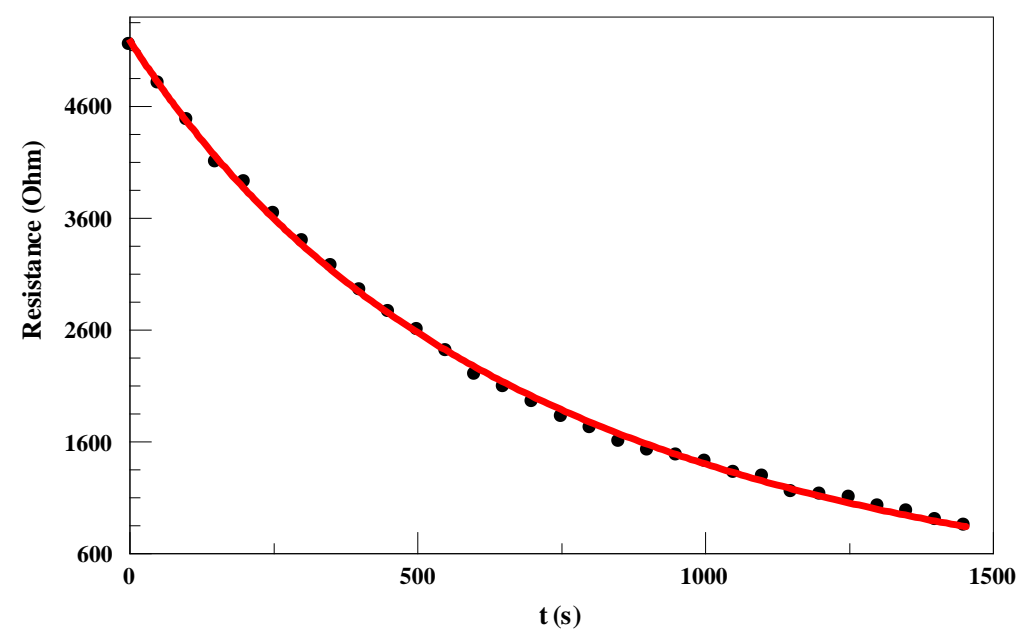

Figure 44: An example of fitting curve follows eq. 3.16: sensor resistance decays as a function of time during the first sensor recovery process

Table 8: Kinetic analysis of response curves during the recovery process.

\begin{tabular}{|c|c|c|c|c|}
\hline & $1^{\text {st }}$ curve & $2^{\text {nd }}$ curve & $3^{\text {rd }}$ curve & $4^{\text {th }}$ curve \\
\hline $\mathrm{k}_{1}\left(\times 10^{-5}\right)$ & $10 \pm 1$ & $9 \pm 1$ & $8 \pm 1$ & $7 \pm 1$ \\
\hline $\mathrm{k}_{2}$ & $0.30 \pm 0.01$ & $0.65 \pm 0.01$ & $0.67 \pm 0.08$ & $0.75 \pm 0.1$ \\
\hline
\end{tabular}

C. Reaction products of recovery process

Detection of $\mathrm{ClO}_{2}$ and $\mathrm{Cl}_{2} \mathrm{O}$ gases as reaction products of the sensor recovery process was also attempted. During the sensor refreshing process, a flow of air through the exposed sensor was captured and collected in an IR gas cell and analyzed by FT-IR spectroscopy. 


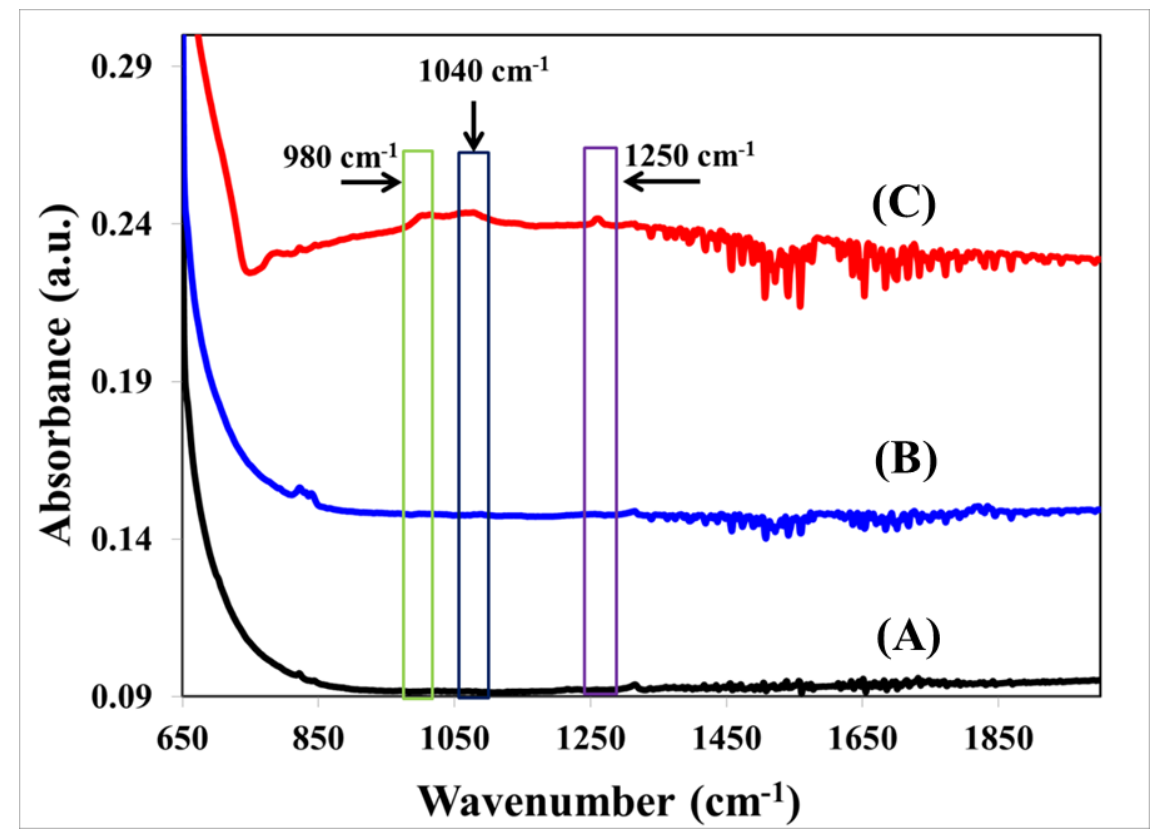

Figure 45: Infrared spectrum composed from blank sample (A), mixture of chorine and air (B), reaction products during sensor recovery process $(\mathrm{C})$

Three additional absorption peaks were observed in IR spectrum of the recovery process sample $(\mathrm{C})$ in the region from $700 \mathrm{~cm}^{-1}$ to $1500 \mathrm{~cm}^{-1}$. These peaks can be ascribed to dichlorine monoxide $\left(\mathrm{Cl}_{2} \mathrm{O}\right)$ and chlorine dioxide $\left(\mathrm{ClO}_{2}\right)$. Specifically, the absorption near $980 \mathrm{~cm}^{-1}$ peak shows a hybrid band $\left(v_{2}+v_{3}\right)$, and the peak at $1250 \mathrm{~cm}^{-1}$ corresponds to overtone $\left(2 v_{1}\right)$ of the vibrational modes of dichlorine monoxide where $v_{1}\left(\sim 630 \mathrm{~cm}^{-1}\right)$ and $v_{2}\left(270 \mathrm{~cm}^{-1}\right)$ are stretching modes while $v_{3}\left(\mathrm{~cm}^{-1}\right)$ is a bending mode [110]. Due to instrument limitations, absorptions below $650 \mathrm{~cm}^{-1}$ were not detected. The origin of these bands could be rationalized from the following reaction:

$$
\mathrm{Cl}_{2}+\mathrm{O}^{-} \stackrel{k_{2}}{\rightarrow} \mathrm{Cl}_{2} \mathrm{O}+e^{-}
$$

Additionally, the $\mathrm{Cl}=\mathrm{O}$ stretching mode of $\mathrm{ClO}_{2}$, which is around $1040 \mathrm{~cm}^{-1}$ is also observed from the result. This is consistent with the observations from many studies on the vibration of the $\mathrm{ClO}_{2}$ molecule [111-113] noted in Table 9 below: 
Table 9: IR observation of dichlorine monoxide $\left(\mathrm{Cl}_{2} \mathrm{O}\right)$ and chlorine dioxide $\left(\mathrm{ClO}_{2}\right)$

\begin{tabular}{|c|c|c|c|}
\hline Molecule & Vibrational Mode & $\begin{array}{c}\text { Experimental } \\
\text { observation }\end{array}$ & Literature values \\
\hline \multirow{2}{*}{$\mathrm{Cl}_{2} \mathrm{O}$} & Overtone of stretching mode & $\sim 980 \mathrm{~cm}^{-1}$ & $977 \mathrm{~cm}^{-1}[110]$ \\
\cline { 2 - 4 } & $\begin{array}{c}\text { Hybrid of stretching and bending } \\
\text { modes }\end{array}$ & $\sim 1250 \mathrm{~cm}^{-1}$ & $1260 \mathrm{~cm}^{-1}[110]$ \\
\hline $\mathrm{ClO}_{2}$ & Stretching mode & $\sim 1040 \mathrm{~cm}^{-1}$ & $\begin{array}{c}1040 \mathrm{~cm}^{-1}[112], 1039 \mathrm{~cm}^{-1} \\
{[111], 1040.2 \mathrm{~cm}^{-1}[113]}\end{array}$ \\
\hline
\end{tabular}




\subsection{Conclusion}

Single crystalline n-doped and Li-doped $\mathrm{SnO}_{2}$ NWs structures exhibit two strong emission bands in their fluorescence spectra. The PL spectra of doped materials also show a red shift of the emission wavelength compared to their undoped counterpart. Raman peaks in the regions from $550 \mathrm{~cm}^{-1}$ to $850 \mathrm{~cm}^{-1}$ were observed and attributed to the $A_{1 g}, B_{2 g}$ and $A_{2 u}$ Raman shifts. The band-gap measurement using optical spectroscopy has shown the dopant induced bandgap narrowing characteristic upon doping, implying higher carrier concentrations of both n- and Li NWs. Availability of this synthetic method for $\mathrm{n}$ - and Li NWs opens up new opportunities for crafting transparent nanoelectronic devices, and sensors.

A thick film chlorine sensor of Sb-doped $\mathrm{SnO}_{2} \mathrm{SW}$ material has been fabricated and demonstrated. Replacing n-doped nanoparticles with n-doped NWs has shown a better sensor performance, in term of sensitivity. The detection limit of chlorine gas has been extended to the ppb level at ambient condition. The chlorine detection sensitivity has been measured and explained in terms of a surface-trap-limit model. It also reconfirmed the role of $\mathrm{Sb}$ dopant used to achieve high sensitivity of $\mathrm{SnO}_{2}$ nanostructures toward $\mathrm{Cl}_{2}$ gas.

Additionally, the mechanisms of sensor response and recovery have been studied and well established. The transient responses of the sensor, i.e. response and recovery of the device as a function of time were kinetically investigated. The sensing mechanism was interpreted in terms of a chemisorption model accompanied by sensor baseline drifting explanation. The occupancy of chlorine atoms in oxygen vacancies of an $\mathrm{SnO}_{2}$ 
lattice, affecting sensor stability, was demonstrated. Dichlorine monoxide $\left(\mathrm{Cl}_{2} \mathrm{O}\right)$ and chlorine dioxide $\left(\mathrm{ClO}_{2}\right)$ are the main products of sensor recovery process 


\section{CHAPTER 4. CARBON NANOTUBES (CNTs)}

In this chapter, some background and significance of carbon nanotubes (CNTs) are reviewed. Following these, studies of synthesis, characterization and sensor performance of as-synthesized MWCNTs for hydrogen gas detection are presented.

\section{1 Background and significance}

\subsubsection{Electronic properties of CNTs}

Carbon nanotubes have a cylindrical structure. The cylinder is formed from graphene - a honey-comb lattice of covalently bonded carbon atoms. There are many possible nanotube (NT) geometries, depending on how graphene is rolled into a cylinder. Subtle geometric variables, such as the alignment between the cylinder axis and the graphene crystal axes, strongly influence the electrical properties of a NT. The structure of CNTs may be completely characterized by the unit chiral vector coordinates $\overline{\mathrm{a}}_{1}$ and $\overline{\mathrm{a}}_{2}$ multiplied by their respective periodicity indices $\mathrm{n}$ and $\mathrm{m}$ and mapped on a graphite lattice (eq. 4.1).

$$
\text { Chiral vector: } \quad \vec{C}=n \overrightarrow{a_{1}}+m \overrightarrow{a_{2}}
$$
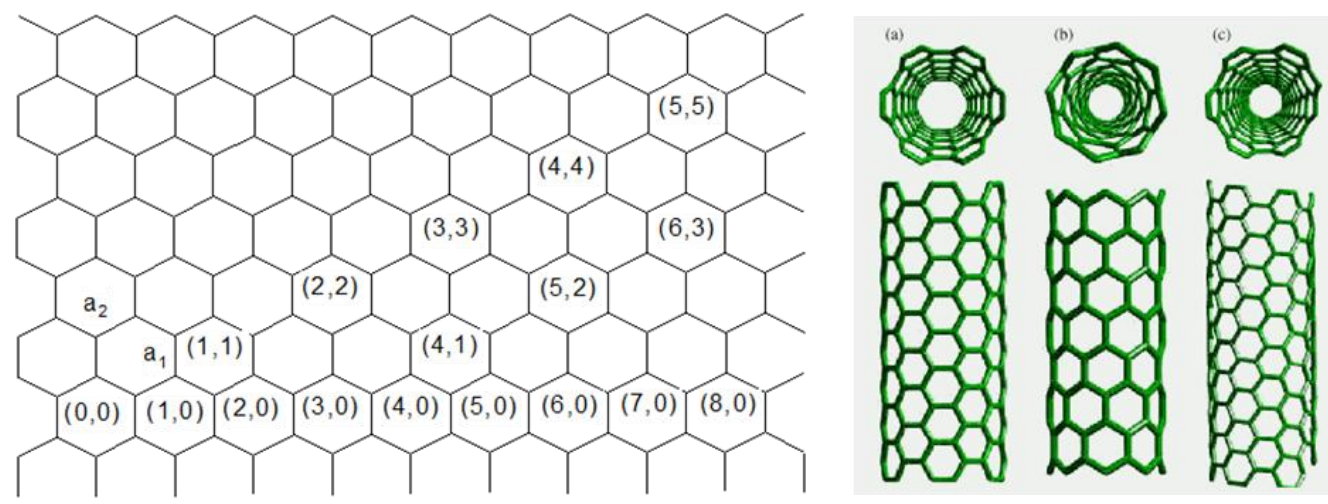

Figure 46: $(n, m)$ coordinate system for a graphene sheet. For values of $n=m$ (a) the corresponding SWNTs are metallic "armchair." SWNTs with $\mathrm{m}=0$ (b) are called "zigzag" and are also metallic. All other combinations (c) are chiral and may be metallic or semiconducting [5]. 
Thus carbon nanotubes are named for their specific $(n, m)$ components of $\bar{a} 1$ and $\bar{a} 2$. Three different types of carbon nanotubes are: armchair, zigzag and chiral. Using the parameters $\mathrm{n}$ and $\mathrm{m}$, one can directly calculate the single wall $\mathrm{CNT}$ diameter using equation 4.2 and the so called chiral angle for chiral CNTs by using eq. 4.3

Tube diameter

$$
\mathrm{d}_{\mathrm{t}}=0.246\left[\frac{\sqrt{\mathrm{n}^{2}+\mathrm{nm}+\mathrm{m}^{2}}}{\pi}\right]
$$

Chiral angle

$$
\phi=\sin ^{-1} \frac{\sqrt{3} m}{2 \sqrt{\left(n^{2}+n m+m^{2}\right)}}
$$

One of the remarkable properties of carbon nanotubes is that they may behave as a conductor or semiconductor as determined by the $(n, m)$ parameters in the tight binding approximation [114]. When $n-m=3 j$ (where $\mathrm{j}$ is an integer,) the valence and conduction bands meet at 6 points in $2 \mathrm{D} \mathrm{k}$-space. These six points are energetically located at the Fermi level and if the wave vector for the first Brillouin zone passes directly through them making a zero band gap or metallic tube. This is also true for the condition where $n$ $=\mathrm{m}$. When $\mathrm{n}-\mathrm{m} \neq 3 \mathrm{j}$ the wave vector for the first Brillouin zone no longer pass through the conduction and valence band contact points. In this case there is a band gap, and the tubes are semiconducting.

It is interesting to note that the metallic nature of a SWNT is not solely dependent on the relative magnitudes of $\mathrm{n}$ and $\mathrm{m}$, but also the tube geometry plays an important role. An experiment by Yildirin and Gulseren [115] at the NIST Center for Neutron Research have shown that by compressing a semiconducting SWNT in to an elliptical shape the 
tube will undergo a transition where the band gap vanishes. At this point the otherwise semiconducting tube shows metallic behavior. Generally the bandgap of CNTs depends inversely on the diameter of the tube, and for CNTs with diameters greater than $14 \mathrm{~nm}$ thermal energy exceeds the bandgap. A consequence of this is that most MWCNTs as well as larger CNTs exhibit metal-like conductivity.

\subsubsection{Growth of CNTs}

Historically, the electric arc discharge is the oldest synthetic method for carbon nanotube production including MWNT and SWNT. Two other synthetic methods are laser evaporation/ablation and chemical vapor deposition (CVD).

The arc discharge technique uses two high-purity graphite electrodes. The anode is graphite containing catalyst metals. The catalysts usually are $\mathrm{Fe}, \mathrm{Ni}, \mathrm{Co}, \mathrm{Mo}, \mathrm{Y}$ either alone or in mixtures. Better results are obtained using bimetallic catalysts. The synthesis is carried out in vacuum chamber containing inert gas at low pressures around 600 mbar. A strong bias is applied across electrodes, which are momentarily brought into contact with each other to strike an arc. The temperature in the inter-electrode zone is so high that carbon sublimes from the positive electrode (anode) and is consumed. For this method, various kinds of products are formed in different parts of the reactor: large quantities of rubbery soot on the reactor walls; web-like structures between the cathode and the chamber walls; grey hard deposits at the end of cathode; and spongy collaret around the cathodic deposits. Amorphous carbon, encapsulated metal nanoparticles, polyhedral carbon are also present in the products $[116,117]$. 


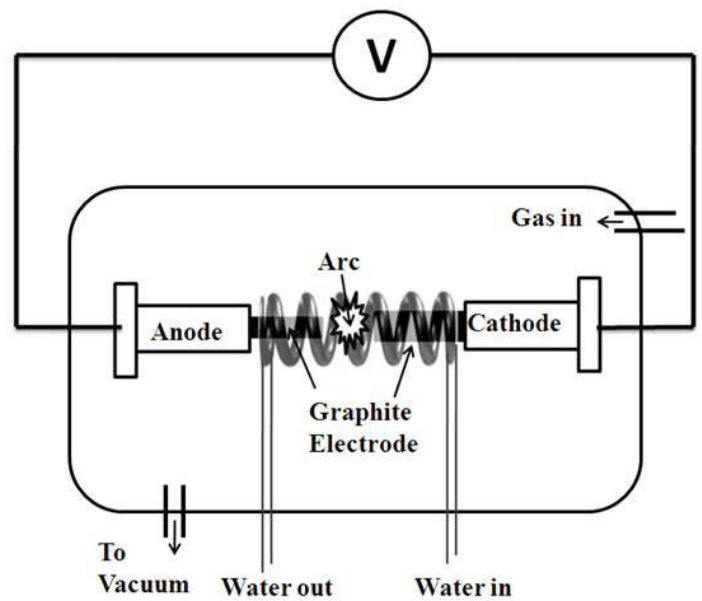

Figure 47: Schematic diagram of a DC-arc discharge system for CNT synthesis [118]

The laser ablation method is technically similar to the arc discharge counterpart. The difference between them is the quality and purity of obtained products. In 1995, Smalley's group [119] at Rice University utilized the pulsed laser-ablation process for the production of single-wall carbon nanotubes. In this method, a laser beam was focused and scanned across a metal-graphite composite target which is placed in a high temperature furnace around $1200^{\circ} \mathrm{C}$. The products were swept by a flow of Argon gas (inert gas) and collected by a water-cooled copper collector positioned downstream. A configuration test has indicated that a composite ablation target consisting of $1 \%$ each of $\mathrm{Ni}$ and Co metal uniformly mixed with graphite gave the best purity of product and highest yield of SWNTs. Impurities in the product were amorphous carbon, graphite particles, fullerenes and metal catalysts. 


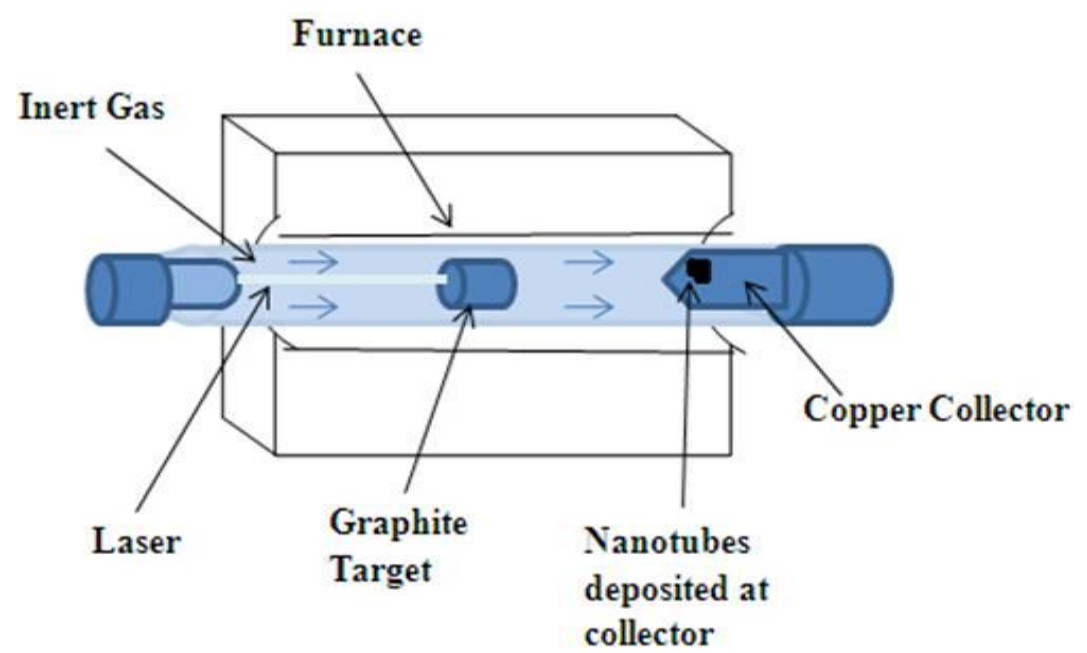

Figure 48: Schematic diagram of a laser ablation system to synthesize CNTs [118]

Along with the capability of producing large quantities of unpurified nanotubes by arc discharge, chemical vapor deposition is another advanced synthetic method of CNT not only in terms of bulk synthesis but also providing control over morphology and location of the synthesized product [120]. Today, the catalytic chemical vapor deposition (CCVD) method is considered as the only economically viable process for large-scale CNT production and the integration of CNTs into various devices [121]. In principle, chemical vapor deposition is the catalytic decomposition of hydrocarbon or carbon monoxide feedstock with the aid of supported transition metal catalysts. Generally, the experiment is carried out in a flow-tube furnace at atmospheric pressure. The reaction mixture containing a source of hydrocarbon and an inert gas (carrier gas) is passed over the catalyst bed at temperatures ranging from $500{ }^{\circ} \mathrm{C}$ to $1100{ }^{\circ} \mathrm{C}$. Ultrafine metal catalyst particles are either introduced into the reactor directly or formed in situ using precursors such as metallic thin films and then annealed to form particles. The general nanotube growth mechanism in CVD process involves the decomposition of hydrocarbon molecules into carbon atoms, which subsequently diffuse into catalysts. The saturation of 
this system leads to the formation of tubular carbon solids with $\mathrm{sp}^{2}$ hybrid structure. The characteristics of the carbon nanotubes produced by the CVD method depend on the working conditions such as the temperature, pressure, concentration and type of hydrocarbon. In addition to the size and nature of metallic catalyst, substrate and reaction time [122] play important roles.

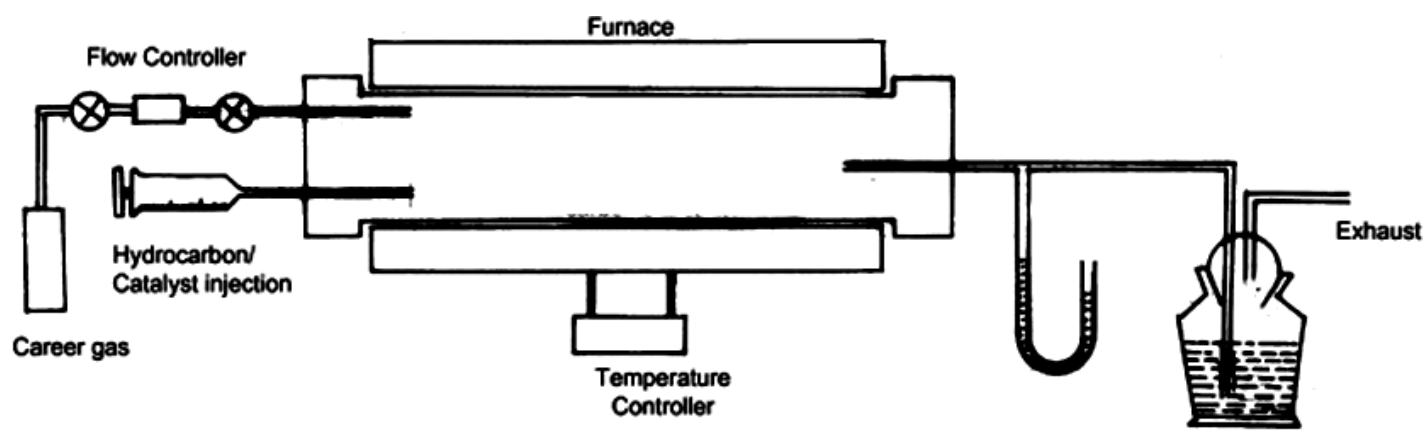

Figure 49: Schematic diagram of a CVD system to synthesize CNTs. Figure was adapted and modified from ref [123]

\subsubsection{Raman Spectroscopy of CNTs}

Raman is one of the most important techniques to characterize CNTs, providing further understanding about properties of this nanomaterial such as structural, optical and electronic properties. Herein, Raman spectroscopy characteristics of CNTs are presented:

\section{A. Single Wall CNT (SWCNT):}

A typical Raman spectrum of a SWCNT is illustrated in Figure 50 below. The most important Raman features of these nanostructures are Raman-active breathing modes (RBMs), the high frequency disordered-induced band (D), graphite band (G band), and second-order Raman scattering from the D-band occurring at twice the frequency of the $\mathrm{D}$ band (2D). 


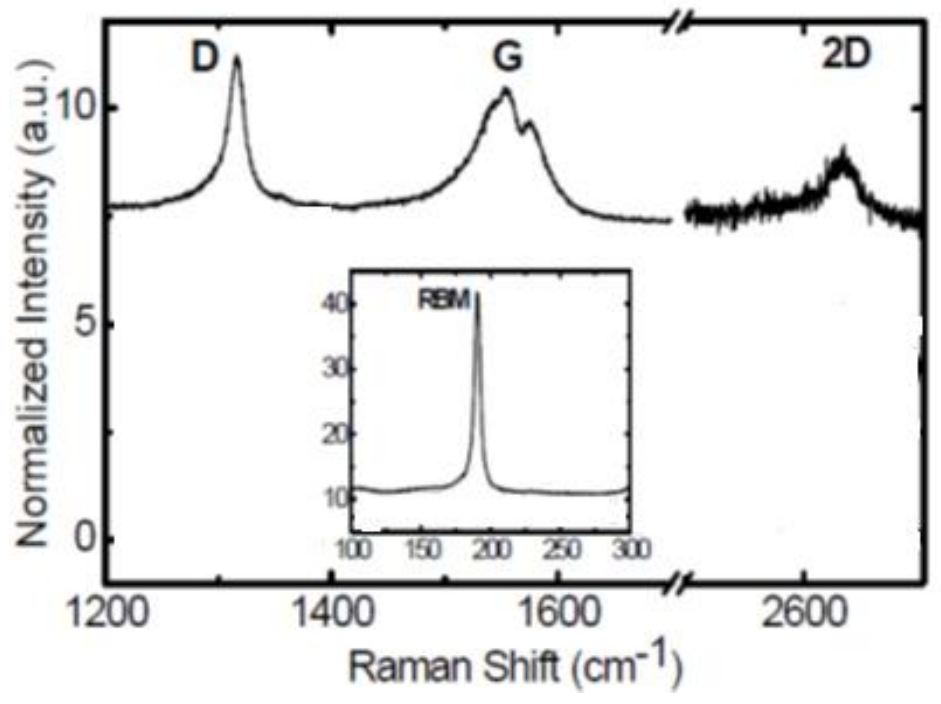

Figure 50: An example of a typical Raman of SWCNTs [32] with 4 bands RBM, D, G and 2D observed in the spectrum.

RBM mode: This mode is a confirmation for the presence of CNTs in the sample and located between 75 and $400 \mathrm{~cm}^{-1}$ from the excitation line. It corresponds to an inphase stretch of carbon along the circumference of a nanotube as illustrated in Figure 51. Therefore, its magnitude changes as a function of the nanotube diameter [124] and can be used to determine the diameter distribution of the nanotubes in a bundle [125]:

$$
\omega_{R B M}=\frac{A}{d_{t}}+B
$$

Where $\mathrm{A}$ and $\mathrm{B}$ are constants and vary between individual tubes

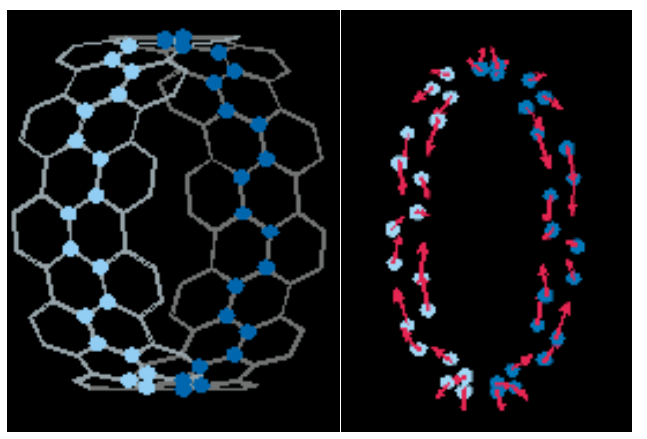

Figure 51: The RBM mode of a SWNT [126]. Small solid circles are carbon atoms in SWNT structure (left) and the arrows indicate the direction of the $\mathrm{C}$-atom displacements (right). 
G-band: The G-band is an intrinsic feature of carbon nanotubes that is closely related to vibrations in all $\mathrm{sp}^{2}$ carbon materials. In simple graphite, a single mode is observed at around $1580 \mathrm{~cm}^{-1}$ [127]. In SWCNT, this mode occurs between $1500-1605$ $\mathrm{cm}^{-1}$ and transforms into two different modes called $\mathrm{G}^{+}$and $\mathrm{G}^{-}$. This is a result of the confinement of wave-vectors along the circumference [128]. The lower frequency component [129] is associated with vibrations along the circumferential direction, and thus depends on the curvature or diameter of nanotube. The higher frequency component $\left(\mathrm{G}^{+}\right)$corresponds to vibrations along the direction of the nanotube axis and does not depend on the diameter of nanotube. These split modes also play an important role in determining whether the nanotube is semiconducting or metallic. Specifically, metallic tubes are easily recognized by the broad and asymmetric line shape of the $\mathrm{G}^{-}$band compared to its counterpart in semiconducting tubes. The $\mathrm{G}^{+}$frequency remains essentially a sharp peak and constant with diameter of either metallic or semiconductor tubes [128].

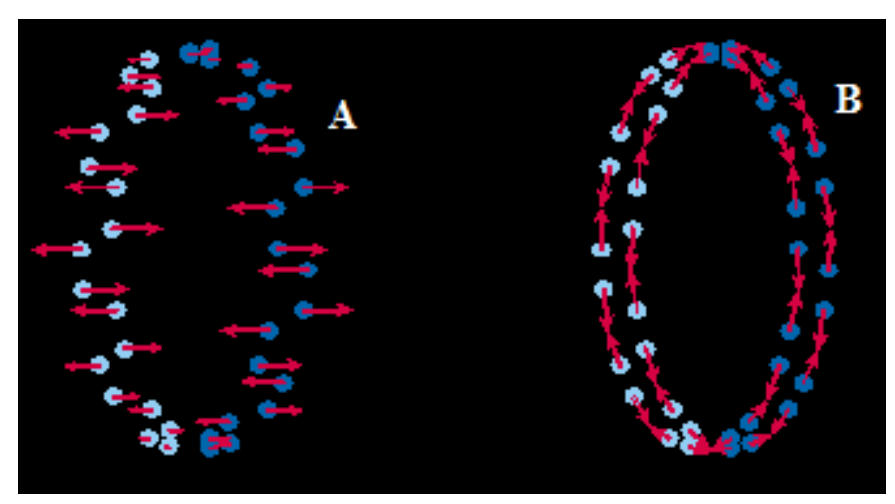

Figure 52: Vibrational modes of $\mathrm{G}^{+}(\mathrm{A})$ and $\mathrm{G}^{-}$(B) bands [126]. Small solid circles and arrows indicate carbon atoms and their vibrational displacement directions, respectively.

The D band and G' (2D) bands: These bands are both observed in the Raman spectra of semiconducting and metallic SWNT. The D band is known as a disordered or 
defect mode because it involves scattering from a defect, which breaks the basic symmetry of a graphene sheet [128]. On the other hand, the second-order G'-band does not require an elastic defect-related scattering process, and it is an intrinsic property of the nanotube and graphite, and present even in defect-free nanotubes for which the D band is completely absent. In Raman spectra, the disordered D-band is located 1250-1450 $\mathrm{cm}^{-}$, and its second-order overtone $\mathrm{G}^{\prime}$-band is $2500-2900 \mathrm{~cm}^{-1}$.

B. Multi-walled Carbon Nanotubes:

Compared to SWCNTs, which are seamless cylinders comprised of only a single layer of graphene, MWCNTs have higher structural complexity. Because MWCNTs consist of multi rolled layers of graphene, most of the characteristic differences that distinguish the Raman spectra in SWCNT from the spectra for graphite are not so evident in MWCNT. For example, the RBM mode of MWCNTs, which associates with the diameter in the case of SWCNT, is usually too weak to be observed and has broad Raman signals. These characteristics result from large diameter outermost tubes and the ensemble average diameters of tubes in MWCNT [130]. Similarly, the G-band in SWCNT is a diameter dependence band mode and splits into $\mathrm{G}^{+}$and $\mathrm{G}^{-}$modes for small diameter tubes. The corresponding splitting modes in MWCNTs are small in intensity and are smeared out due to the effect of the diameter distribution within individual MWCNT. Therefore, the G-band of MWCNTs in Raman spectra is observed as a single broad peak around $1582 \mathrm{~cm}^{-1}$, just like in graphite [130].

\subsubsection{Doping of CNTs}


It is well known that CNTs have a very wide range of application in almost every field of nanotechnology. One of the most crucial applications of this nanomaterial is in nanoelectronics, requiring $\mathrm{n}$ and $\mathrm{p}$ type doping, which can be attained by doping with electron donors and electron acceptors. Many routes to achieve the dopant containing CNTs are listed in Figure 53.

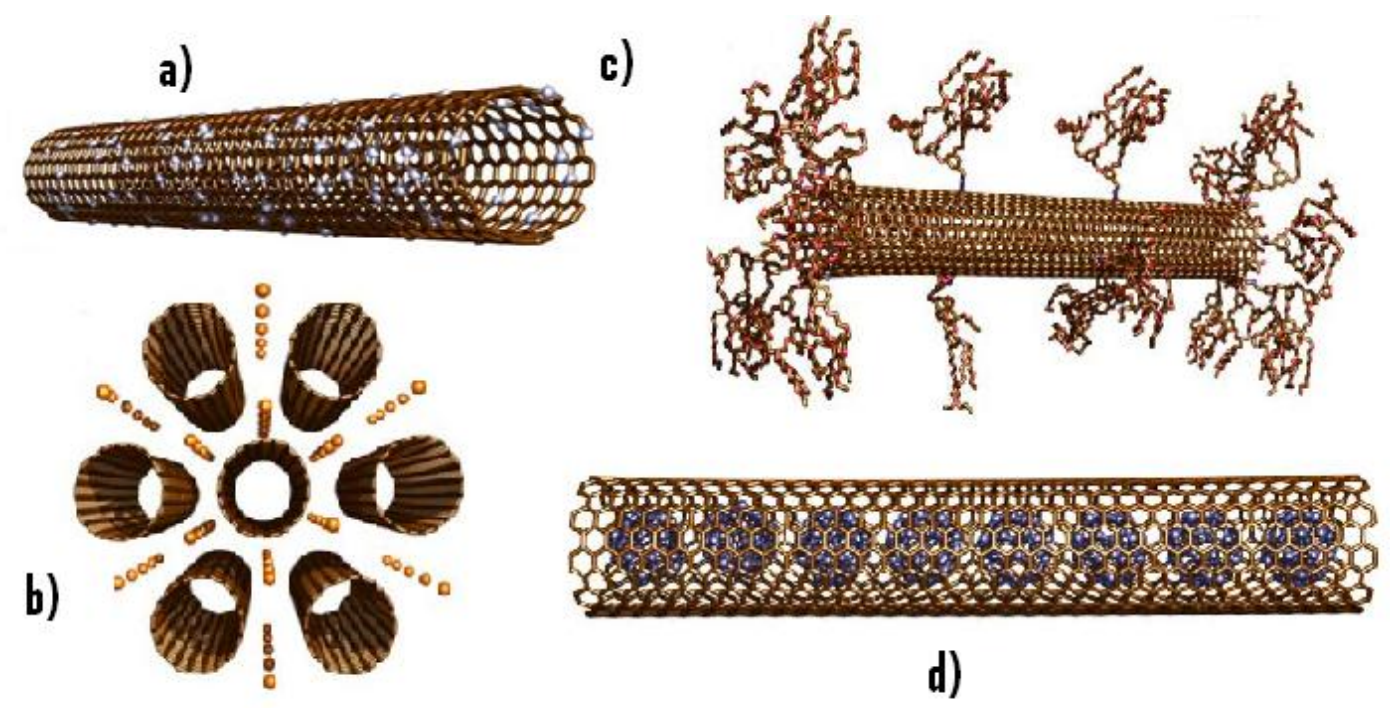

Figure 53: Doping CNTs through many different pathways. The figure is adapted and slightly modified from ref [131]

a) Substitutional doped single-wall nanotubes through post synthesis modification of CNT with dopant containing organic molecule or incorporation during the synthetic process

b) Nanotube bundles intercalated with atom or ions

c) CNTs are functionalized with dopant containing molecules that change the properties of CNTs

d) CNTs are filled with fullerenes and inorganic compounds 
However, conventional pathways to manipulate doping in semiconducting CNTs are based on the intercalation of alkaline metals such as $\mathrm{K}, \mathrm{Rb}$ [132] [133] or Li [134, 135] for n-type doping. Besides, accumulation in the form of carbon-halogen complex using fluorine [136] or bromine [137] is also reported. More recently and commonly, studies on boron and nitrogen substitutionally doped CNTs have showed that their electronic properties strongly depend on the composition, where the dopants including nitrogen and boron tend to produce electronic properties of metallic CNTs [138-141].

The most common pathway to dope CNTs is through classical CVD and aerosol assisted CVD methods. The doping is routinely carried out in situ, in a CVD reactor or enclosed chamber, by flowing volatile gases containing dopants such as acetonitrile [142], $\mathrm{NH}_{3}$ [143], melamine [144], tri-isopropyl borate [145] etc., via mass flow regulators that control the precise flow rate. The overall cost of system complexity and handling toxic gases are large obstacles for the synthesis of doped-CNTs, and these methods are considered to be "non-green approach".

\subsubsection{Carbon nanotube based gas sensors}

Kong and coworkers [14] demonstrated that upon exposure to gaseous molecules such as nitrogen dioxide $\left(\mathrm{NO}_{2}\right)$, ammonia $\left(\mathrm{NH}_{3}\right)$ or oxygen $\left(\mathrm{O}_{2}\right)$ conductivity of semiconducting SWCNTs are significantly altered with high sensitivity and fast response time. Modi's group [146] has developed better sensors that have superior selectivity and are insensitive to effects of environmental conditions such as moisture, temperature and gas flow rate. They used thin devices utilized thin film of MWCNTs and employed electrical break down voltage for sensing different gases on the tips of nanotubes. In 
another study, Jang et al [129] developed a chemical sensor employing MWCNTs as the active sensing element for detection of $\mathrm{NH}_{3}$ in air at different flow rates. Further studies for other gas detections were also achieved such as the work done by Penza's group [147]. In this work, vapors of volatile organic compounds were detected at ppm level concentrations by a CNT-coated surface acoustic wave sensor.

In general, the CNT sensing mechanism of many gases is based on the charge transfer between defect sites on CNTs and the adsorbed analyte. The transfer of either electrons or holes from absorbed molecules to CNT or vice versa will change the conductance of CNTs. Previous theoretical studies have indicated that due to the weak CNT-analyte adsorption interaction in defect free CNTs leads to a large activation barrier for charge transport for $\mathrm{NH}_{3}, \mathrm{CO}$ or $\mathrm{NO}_{2}$ gases even at high temperatures [14, 148, 149]. However, the adsorption of gas molecules is much easier on defective CNTs $[150,151]$ which leads to lower adsorption barrier and promotes the charge transfer rate. Oxygen is known to adsorb and dissociate on the defective sites of CNTs. Furthermore, dissociated oxygen extracts an electron from CNT doping it to a p-type semiconductor [152]. This is because the reduction potential of oxygen is lower than the commonly accepted Fermi level in CNTs as will be discussed later. The concentration of the ionized oxygen can be influenced by reducing as well as oxidizing gaseous species thereby affecting conductivity of CNTs. Multiple oxygen molecules could potentially dissociate on the same CNT defect, providing more dissociation sites for gaseous molecules and leading to much higher net charge transfer to CNTs [153]. 


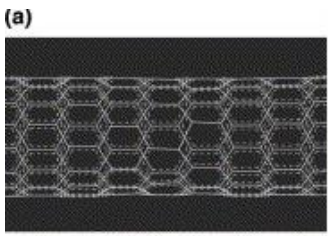

(d)

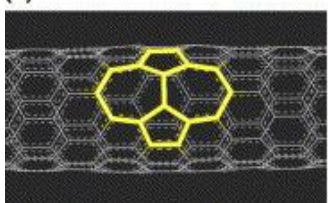

(b)

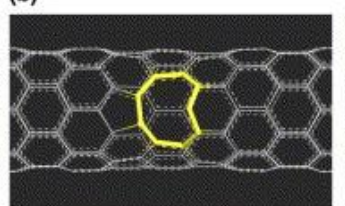

(e)

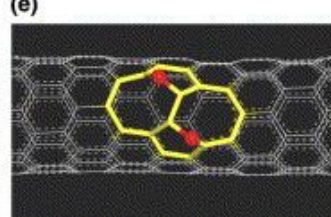

(c)

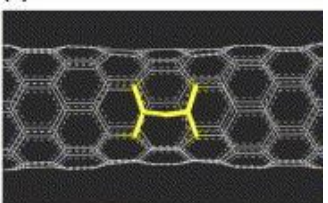

(f)

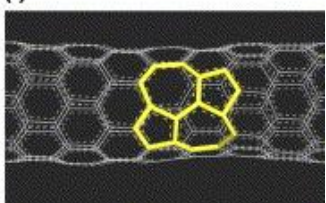

Figure 54: Various structural defects on CNT including defect-free tube (a), vacancy (b), interstitial (c), straight Stone-Wales (d) $\mathrm{O}_{2}$ molecule pre-dissociated defect (e), and slanted stone-Wales defect (f)

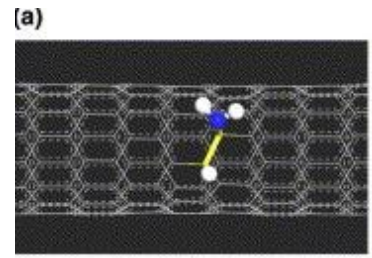

(d) (b)

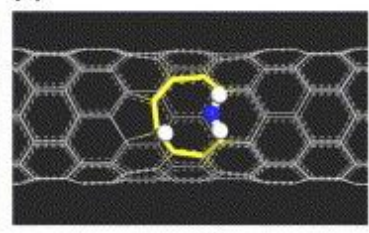

(e)

(c)
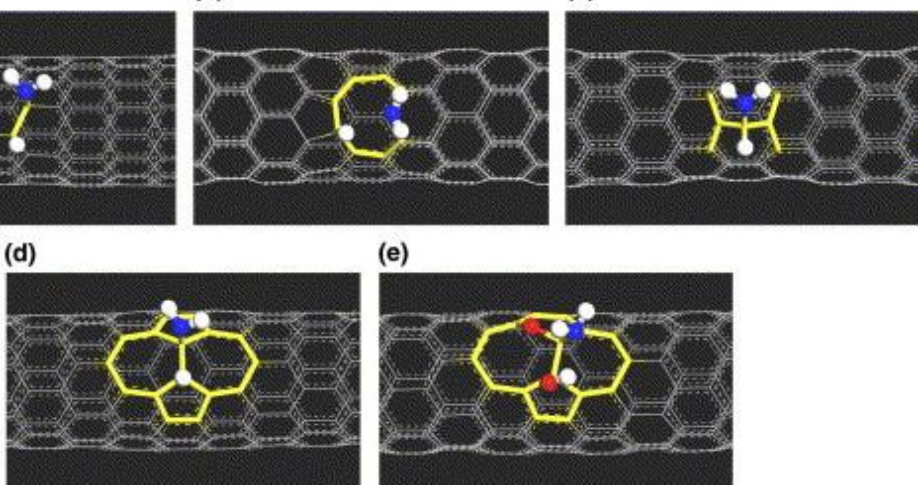

Figure 55: An example of $\mathrm{NH}_{3}$ molecule adsorption on defective CNT for different types of defect illustrated on figures above.

Work presented here shows a new method to synthesize CNTs and also a mean of derivatizing them for use as sensors. The primary focus here is understanding the detailed molecular mechanism of analyte and CNT interactions. 


\subsection{Results and Discussions}

\subsubsection{Electron microscopy}

A. Ni nanoparticle precursor synthesis:

In CNT synthesis methods (A and B; see chapter 2) we generate Ni nanoparticles on substrate by annealing a thin film of $\mathrm{Ni}$ deposited through physical vapor deposition. The effect of catalyst layer thickness to nanoparticle formation was explored. As the thickness of Ni film changes, the morphology of nanoparticles also changes. Figure 56 below shows SEM images of Ni nanoparticle catalysts, annealed from two different Ni film thicknesses. As analyzed from the images, when the annealing film thickness increases from $5 \mathrm{~nm}$ to $20 \mathrm{~nm}$, the nanoparticle size increases from $50 \pm 20 \mathrm{~nm}$ to $340 \pm 150$ nm. In addition, formation of completely separated individual particle cannot be achieved if the Ni film is too thick. This has been observed in the $20 \mathrm{~nm}$ thick Ni film case where the film still remains with generated catalysts trapped inside the film after the annealing process.

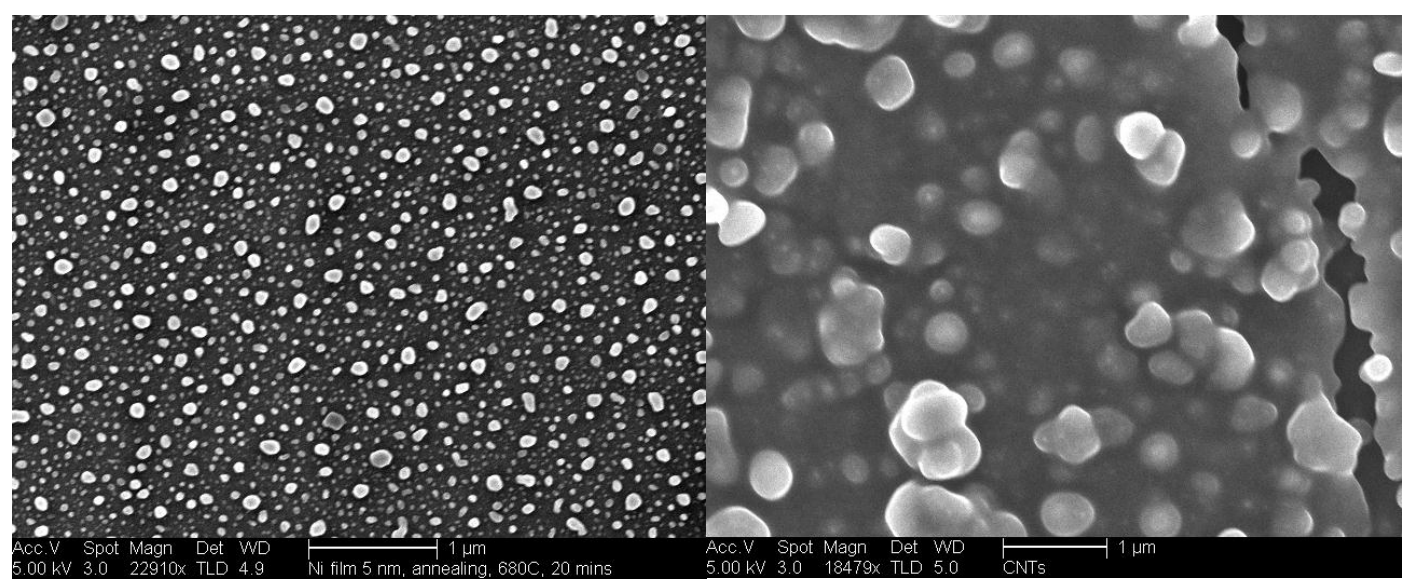

Figure 56: Formation of Ni nanoparticle catalysts after annealing 5nm and 20nm thick Ni thin films at $680^{\circ} \mathrm{C}$. 


\section{B. CNT synthesis}

Method A: The synthesis of CNTs was carried out using a $5 \mathrm{~nm}$ thick Ni thin film to form nanoparticle precursors. From dual chamber synthesis (method A described in chapter 2, section 2.2.1), different results were observed at different synthesis temperatures. Formations of carbon nanotube/nanowhisker like structures using $\mathrm{Ni}$ nanoparticle catalysts took place at temperatures between $600^{\circ} \mathrm{C}$ and $610^{\circ} \mathrm{C}$ with best results at $600^{\circ} \mathrm{C}$. Lower or higher synthetic temperatures resulted in non-CNT products. It was also observed that the diameter of CNTs increases as the reaction temperature increases. At $590^{\circ} \mathrm{C}$ nucleation of CNT appears to collapse. On the other hand, at much higher temperature, $620^{\circ} \mathrm{C}$ carbon nanoparticles were generated.

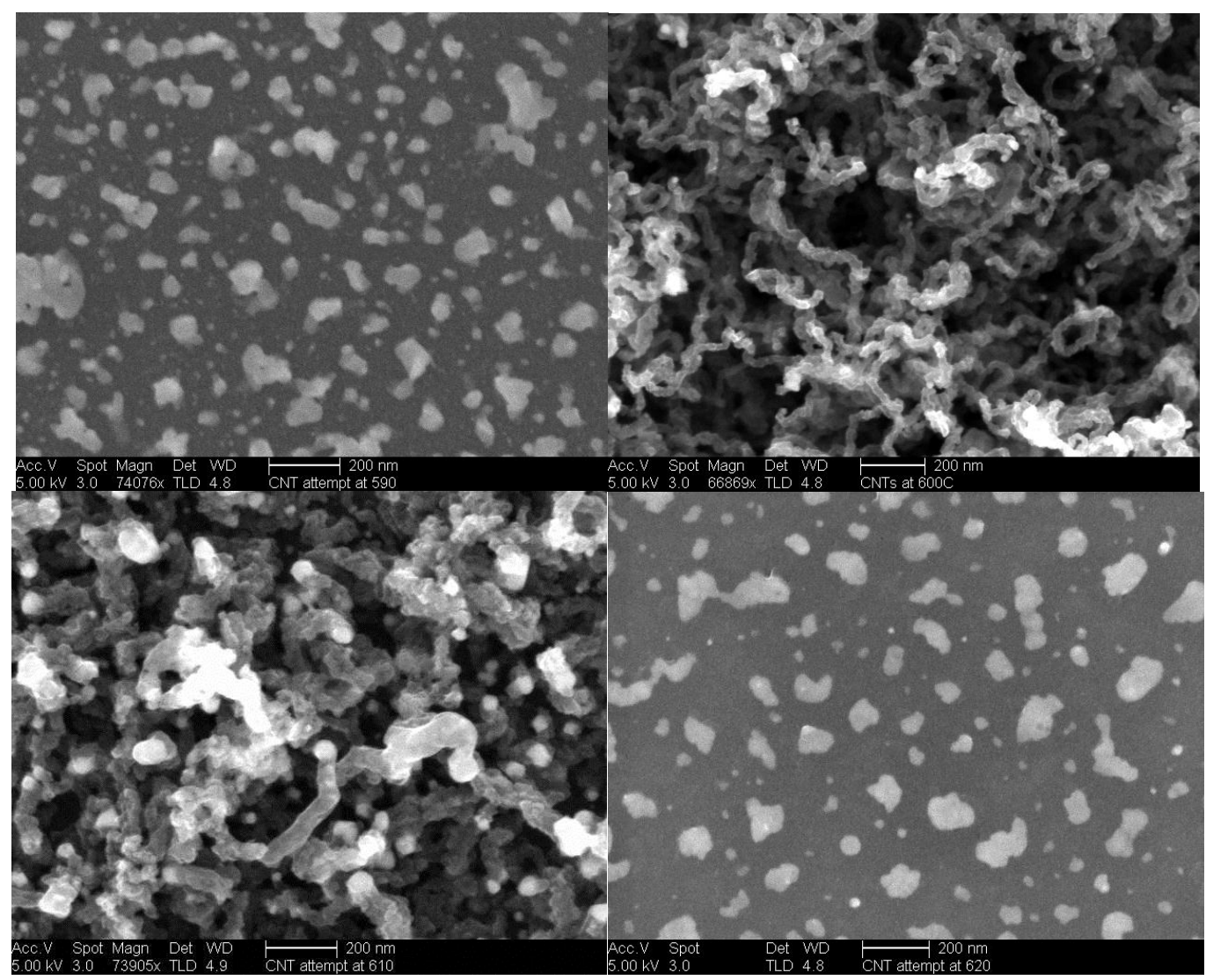

Figure 57: Method A for the synthesis of CNTs at $590^{\circ} \mathrm{C}, 600^{\circ} \mathrm{C}, 610^{\circ} \mathrm{C}$ and $620^{\circ} \mathrm{C}$ 
These observations could be rationalized using a model developed for silicon nanowire/nanoparticle synthesis by Chan [154] and it is illustrated below.

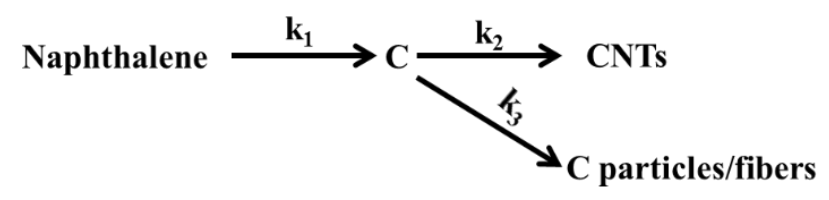

The model assumes that decomposition of naphthalene results in the formation of an unstable intermediate of carbon (C). The concentration of carbon could be consumed in two competing pathways, either CNTs or C particles/fibers. Therefore, there are three dominant reaction rates affecting the formation of products, including the rate of naphthalene decomposition $\left(\mathrm{R}_{1}\right)$, the rate of CNT $\left(\mathrm{R}_{2}\right)$ and the rate of carbon nanoparticle growth $\left(\mathrm{R}_{3}\right)$.

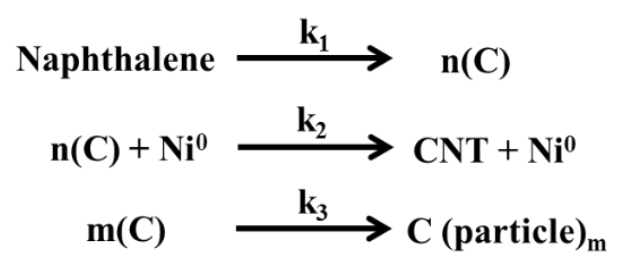

At the elevated temperature, $\mathrm{k}_{1}$ is large and not a rate determining step. The rate constants $\mathrm{k}_{2}$ and $\mathrm{k}_{3}$ are inherently temperature dependent and change with temperature. Thus, the dominating product depends on the ratio of $\mathrm{k}_{2}$ to $\mathrm{k}_{3}$.

The rate of formation of CNTs can be written for equation as:

$$
R_{2}=\frac{d[C N T]}{d t}=k_{2}\left[N i^{0}\right] .[C]^{n}
$$

The rate of formation of $\mathrm{C}$ particle can be written for equation as:

$$
R_{3}=\frac{d[C(\text { particle })]}{d t}=k_{3} \cdot[C]^{m}
$$

The ratio of the rate of reactions is: 
$\frac{R_{2}}{R_{3}}=\frac{k_{2}\left[N i^{0}\right] \cdot[C]^{n}}{k_{3} \cdot[C]^{m}}=K \cdot[C]^{n-m}$

If $\mathrm{m} \gg>\mathrm{n}$;

$\frac{R_{2}}{R_{3}}=K \cdot \frac{1}{[C]^{m}}$

Therefore, high carbon concentration in the vapor phase at high temperature leads to $\mathrm{R}_{2}<<\mathrm{R}_{3}$ meaning that the rate of $\mathrm{C}$ particle formation would dominate the rate of CNT formation. In other words, the heterogeneous nucleation rate would exceed the rate of nanotube growth mediated through $\mathrm{Ni}$ nanoparticles. On the other hand, at lower temperatures, relative low carbon concentration in the vapor leads to $R_{2}>>R_{3}$, resulting in a higher density of CNTs and lower quantity of C particles.

Since the atomic rearrangement is the main the characteristic of CNT growth mechanism, which involves a thermally activated elementary process, the growth is diffusion controlled, and thus its rate is temperature dependent, according to the Arrhenius equation (eq. 4.9). The rupture carbon structures at $590^{\circ} \mathrm{C}$ could be associated with the slowdown of growth rate at such lower temperatures.

$$
D=D_{0} e^{-E_{A} / k T}
$$

Where

$D$ and $D_{0}$ are the diffusion coefficient at temperature $\mathrm{T}$ and at infinite temperature $E_{A}$ is the activation energy for diffusion

$k$ is the Boltzmann constant

In order to confirm the formation of CNTs which have the hollow structure, high resolution TEM studies were undertaken. The data below show the morphology of assynthesized products at $600^{\circ} \mathrm{C}$. From Figure 58, the diameter of CNTs is about 20nm with 
length of a few microns. It also shows that there are about 20 to 30 concentric shells within each MWCNT. The MWCNT growth mechanism was the tip based as confirmed from EDX elemental composition analysis showing Ni nanoparticles at the tip of the CNT.
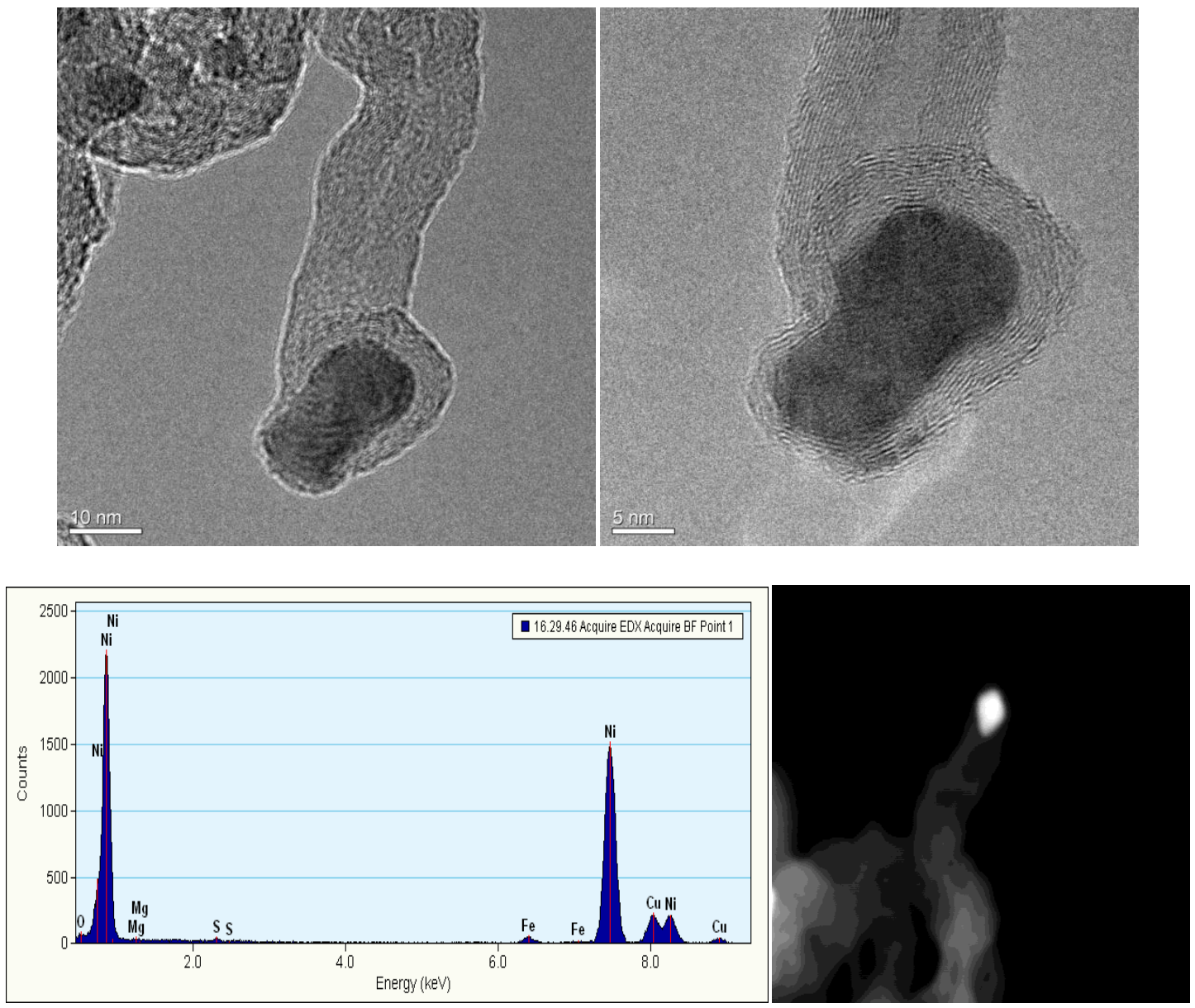

Figure 58: HRTEM of CNT synthesized at $600^{\circ} \mathrm{C}$ in $2 \mathrm{hrs}$ (top) and EDX of catalyst (bottom) and STEM of a CNT.

Method B: In this method, the catalysts and carbon source (naphthalene) were heated up to the desired temperature simultaneously in single glass chamber. Similar to the previous process using dual chamber synthesis, four different products were observed at four different temperatures. The wire-like products were formed at $600^{\circ} \mathrm{C}$ and $610^{\circ} \mathrm{C}$. 
At a lower temperature $590^{\circ} \mathrm{C}$ and higher temperature $620^{\circ} \mathrm{C}$, carbon sheathed nanoparticles were observed

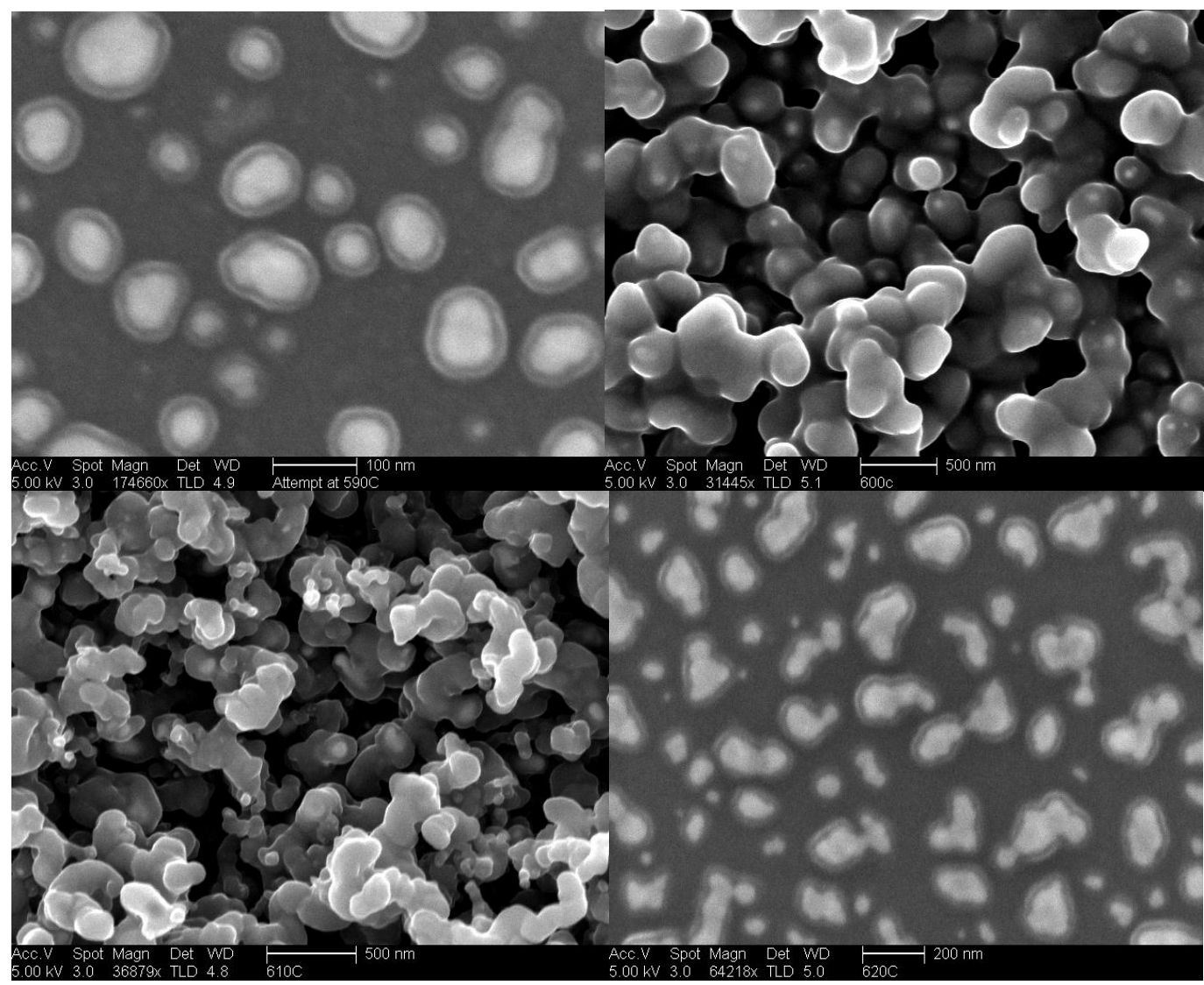

Figure 59: Method B for the synthesis of CNTs in uncontrolled manner at $590^{\circ} \mathrm{C}, 600^{\circ} \mathrm{C}, 610^{\circ} \mathrm{C}$ and $620^{\circ} \mathrm{C}$

Method C: Optical images of glass tubes shown below are from results of synthesis, using ferrrocene and cobaltocene as catalysts with naphthalene as a carbon source. Black powder like residue coated the glass tube walls. The temperature range, monitored by a temperature probe along the glass tube, indicated that CNTs just grow in the temperature window from $590^{\circ} \mathrm{C}$ to $620^{\circ} \mathrm{C}$. This range was comparable with the observation in the case of Ni nanoparticle catalysts, annealed from thin film (Figure 56) using methods A and B. Further characterizations were carried out by SEM with the data below showing the observation of non-optimized products of CNT synthesis. SEM 
images also revealed the presence of catalyst nanoparticles ( $\mathrm{Fe}$ and $\mathrm{Co})$ at the tip of CNTs. With identical growth conditions including temperatures and pressures (same amounts of precursors), cobaltocence as the catalyst precursor provided smaller diameter CNTs with higher density. Besides CNTs, carbon fiber-like nanostructures of larger diameters were also present in the products.

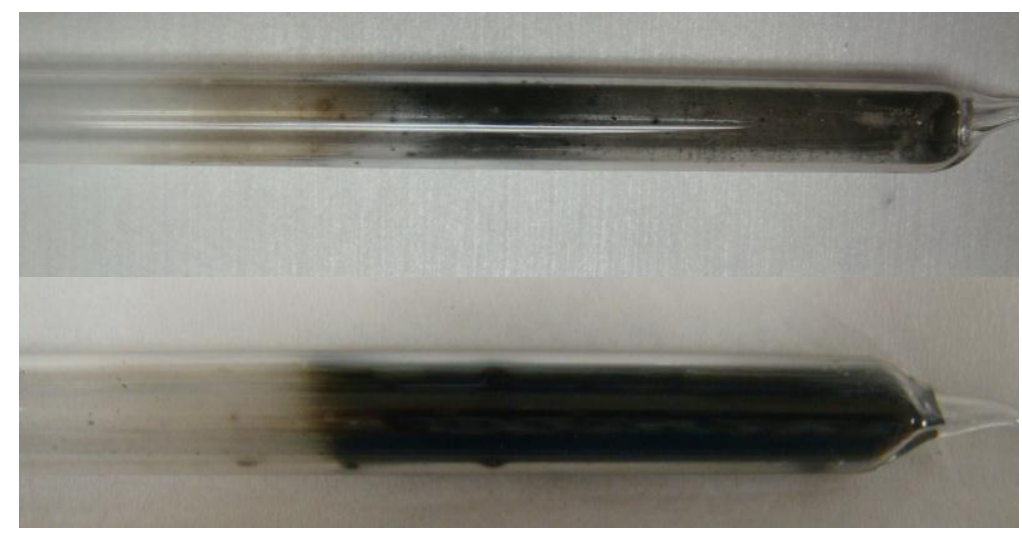

Figure 60: Optical images of method C for the synthesis of CNTs ferrocene (top) and cobaltocene (bottom). Synthesis using cobaltocene gives higher density of CNT products.

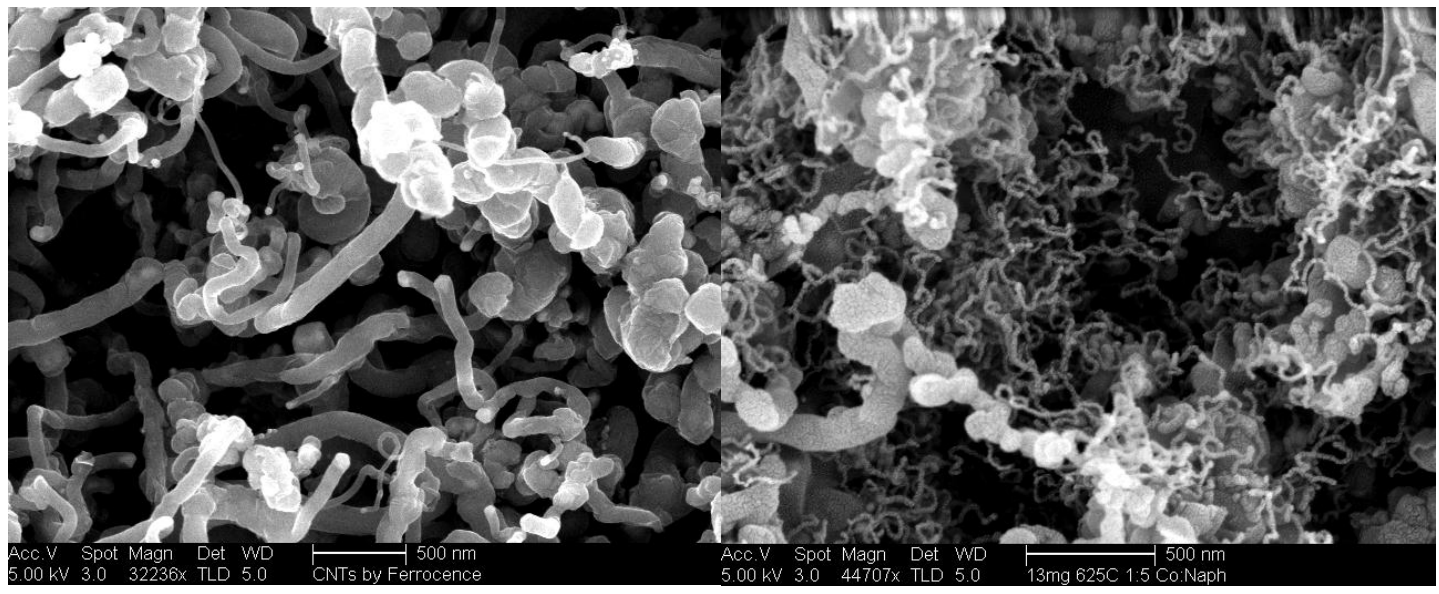

Figure 61: SEM images of CNTs by method C: ferrocene (left) and cobaltocene (right). Smaller diameter CNTs were observed using cobaltocene.

Further confirmation of CNT formation was carried out using HR-TEM.

Similarly, tip growth mechanism using cobaltocene as catalyst precursor was observed in Figure 62. The image shows 20 to 30 concentric CNTs in each MWCNT. More 
importantly, CNTs, grown by this method, seem to be highly defective. It has been confirmed that the substrate material, its surface morphology and textural properties greatly affect the yield and quality of the resulting CNTs. The chemical state and structure of the substrate are more important than that of the metal catalyst [155]. The weak metal ( $\mathrm{Ni}$ catalysts)-support (silica substrate) interaction allows high metal dispersion, resulting in metal catalyst aggregation and a high density of catalytic sites [156]. This could facilitate the growth of more defective CNTs and carbon nano-fiber [157].

However, defective characteristic of the as-synthesized CNT product plays an extremely crucial role in the sensing application, which is based on the electrical response in the presence of chemical vapor. The defect sites can dominate the electrical response by forming low energy adsorption sites that also serve as sites for analyte condensation [151]. Therefore, defective CNTs synthesized from this method are employed for the sensing application.

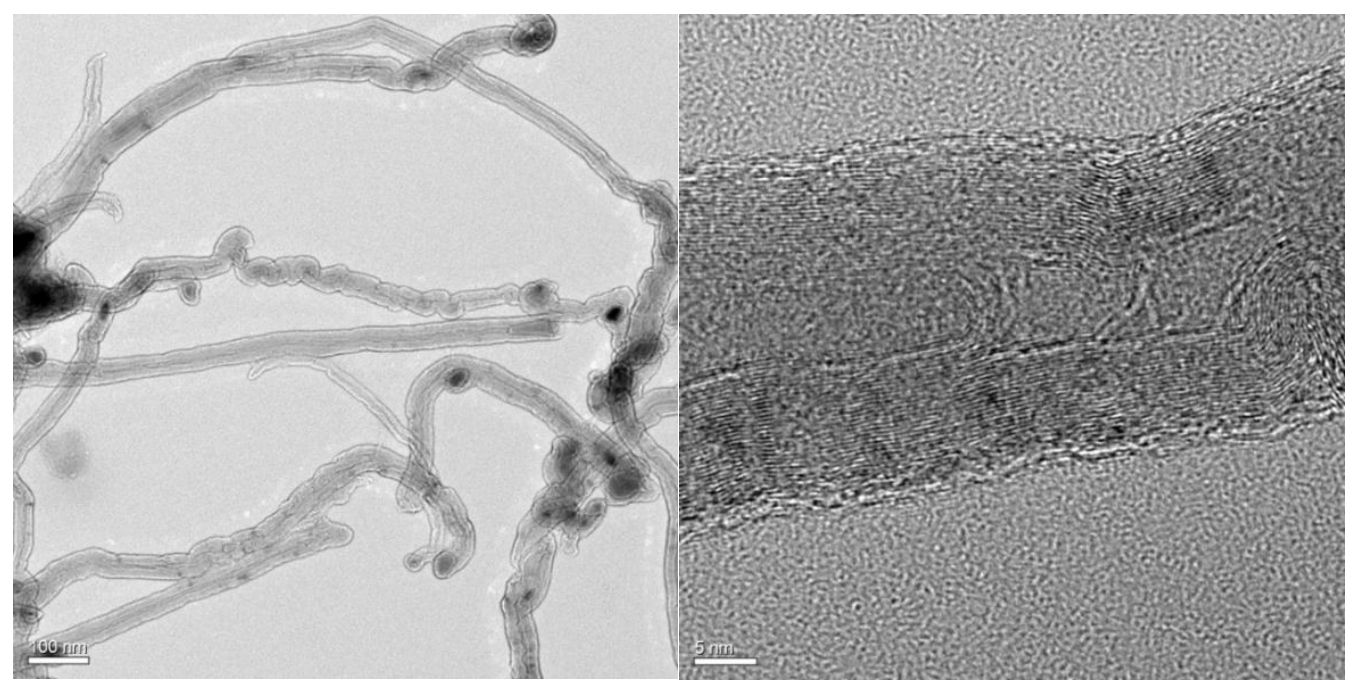

Figure 62: TEM images of CNTs by method C using cobaltocene as catalyst precursor. Tip-growth mechanism and about 20-30 concentric CNTs in each MWCNT are observed. 


\subsubsection{Raman Characterization of undoped and doped CNTs}

Raman spectroscopy was employed to investigate the structure of as-synthesized undoped and doped CNTs [158]. Different n-type and p-type doping concentration CNT samples were collected and characterized. The data was analyzed to confirm the insertion of impurities (dopants) into the lattice structure of CNTs upon doping. In this experiment, five different samples undoped, 5\% p-doped, $10 \%$ p-doped, 5\% n-doped and 10\% ndoped CNTs were investigated

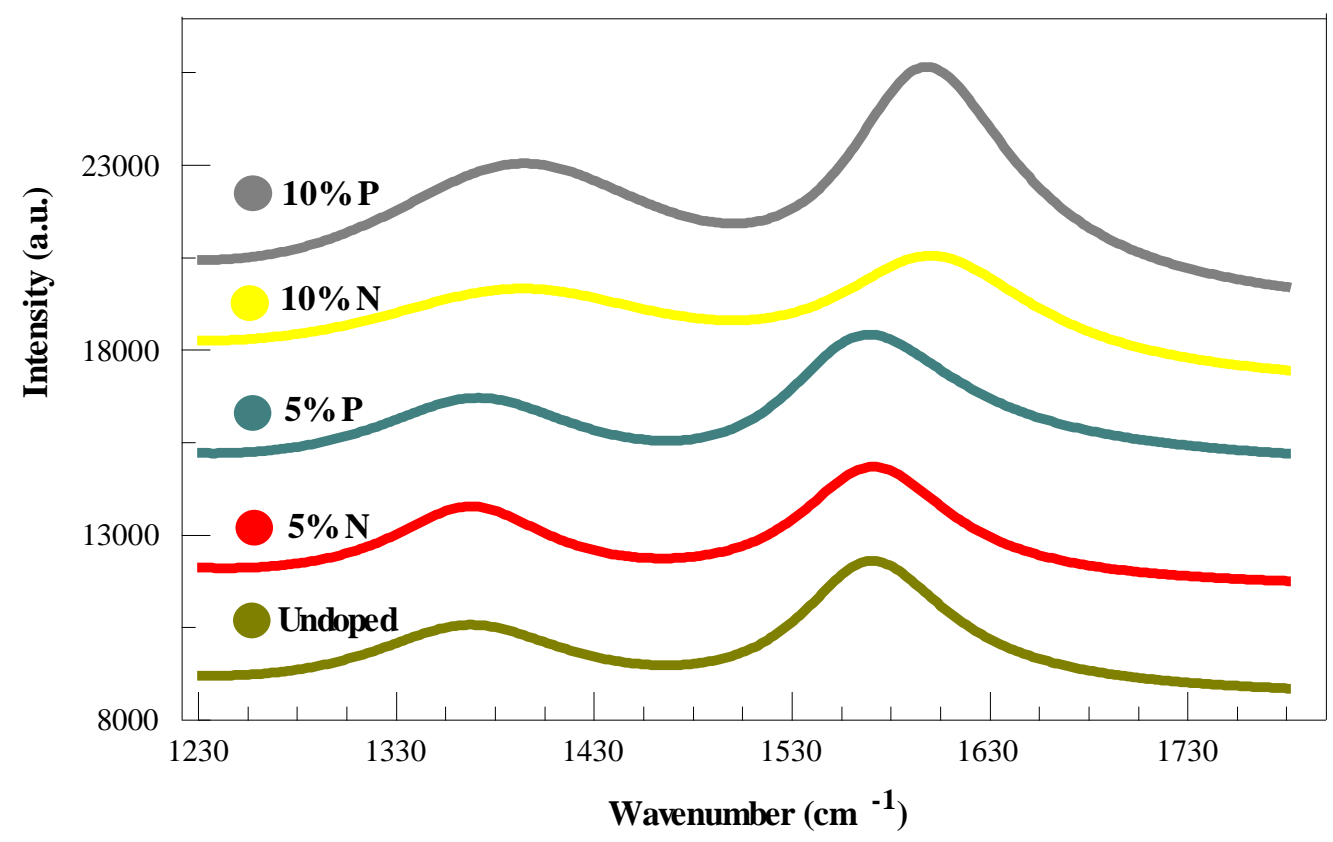

Figure 63: Raman spectroscopy of as-synthesized MWCNTs in the region 1200-1800 cm ${ }^{-1}$. Two peaks corresponding to D band and $\mathrm{G}$ band are observed. The data was fitted following eq. 4.10. The curves are shifted vertically to better display the results.

The curve fitting was based on two Lorentzians and one linear baseline:

$$
Y=\frac{I_{1}}{1+B_{1}\left(X-X_{01}\right)^{2}}+\frac{I_{2}}{1+B_{2}\left(X-X_{02}\right)^{2}}+C X+D
$$

Where

$\mathrm{X}$ and $\mathrm{Y}$ : wavenumber and intensity, respectively. 
I: peak intensity

B: Reciprocal of peak FWHM (full width at half maximum)

$\mathrm{X}_{0 \mathrm{i}}$ : peak positions

C, D: fitting constants for modeling background

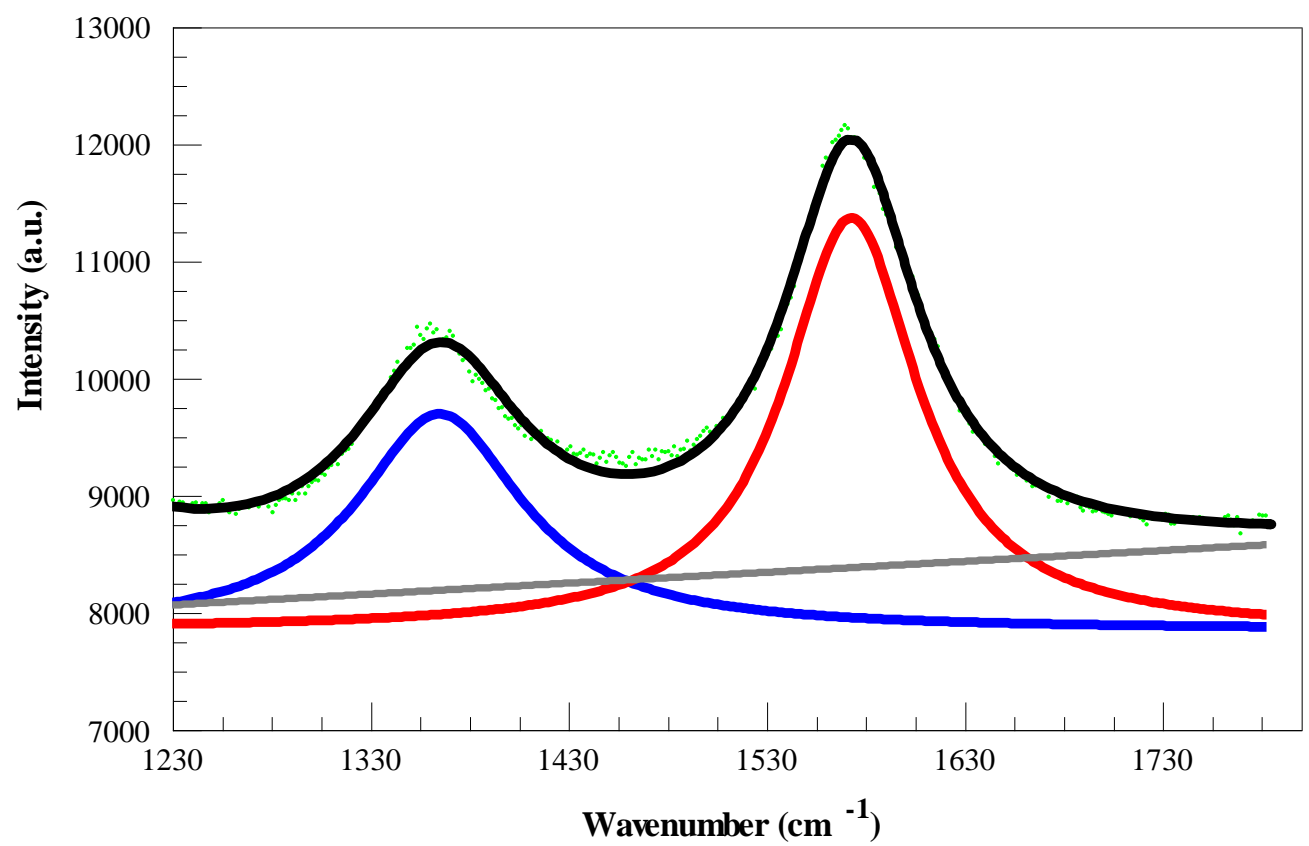

Figure 64: An example of fitting using equation (eq. 4.10) using the undoped CNT sample data. Blue, red, grey and black cures correspond to D band, G band, linearly tilted base line and entire Raman spectrum of sample, respectively.

Table 10: The non-linear least squares analysis of $\mathrm{D}$ and $\mathrm{G}$ bands from Raman spectroscopy of assynthesized MWCNTs

\begin{tabular}{|l|l|l|l|l|l|}
\hline & Undoped & $\mathbf{5 \%}$ & $\mathbf{1 0 \%}$ & $\mathbf{5 \%}$ & $\mathbf{1 0 \%}$ \\
\hline $\mathrm{I}_{\mathrm{D}} \pm 3 \sigma$ & $1970 \pm 30$ & $2180 \pm 50$ & $4250 \pm 70$ & $2140 \pm 20$ & $2560 \pm 20$ \\
\hline $\mathrm{I}_{\mathrm{G}^{ \pm}} 3 \sigma$ & $3600 \pm 30$ & $3560 \pm 40$ & $6210 \pm 50$ & $3220 \pm 20$ & $3210 \pm 20$ \\
\hline $\mathrm{B}_{\mathrm{D}} \pm 3 \sigma\left(\times 10^{4}\right)$ & $2.4 \pm 0.1$ & $2.2 \pm 0.1$ & $1.0 \pm 0.1$ & $1.8 \pm 0.1$ & $1 \pm 0.1$ \\
\hline $\mathrm{X}_{0 \mathrm{D}^{ \pm}} 3 \sigma\left(\mathrm{cm}^{-1}\right)$ & $1368 \pm 1$ & $1371 \pm 1$ & $1394 \pm 1$ & $1368 \pm 1$ & $1392 \pm 1$ \\
\hline $\mathrm{X}_{0 \mathrm{G}^{ \pm}} 3 \sigma\left(\mathrm{cm}^{-1}\right)$ & $1567 \pm 1$ & $1558 \pm 1$ & $1594 \pm 1$ & $1569 \pm 1$ & $1598 \pm 1$ \\
\hline $\mathrm{I}_{\mathrm{D}} / \mathrm{I}_{\mathrm{G}} \pm 3 \sigma$ & $0.54 \pm 0.04$ & $0.61 \pm 0.06$ & $0.68 \pm 0.05$ & $0.66 \pm 0.03$ & $0.80 \pm 0.03$ \\
\hline
\end{tabular}

Note: $\sigma$ is standard deviation. Subscripts D and G denote D and G band, respectively. 
Raman spectra in the $1200-1800 \mathrm{~cm}^{-1}$ region of multiple doped and undoped MWCNTs samples are showed in Figure 63 with peak positions and analysis summarized in Table 10. The first peak is located around $1370 \mathrm{~cm}^{-1}$, denoting disorder-induced in graphitic material D band. The intensity of this peak is strongly related to the impurities and defects present in CNT structure. The feature with the frequency around $1580 \mathrm{~cm}^{-1}$ indicates the graphitic $\mathrm{G}$ band, which relates to a lattice tangential vibration of CNTs. To determine D and G band frequency, the raw data of each Raman curve is fitted using two Lorentzians and linear baseline. An example illustrated in Figure 64 showing their relative contributions.

Another Raman peak was found in the region $2500-2900 \mathrm{~cm}^{-1}$ depicted in Figure 65 with peak positions and analysis summarized in Table 11. This is the second-order Raman peak named G', which locates at $\sim 2700 \mathrm{~cm}^{-1}$. G' band is an overtone mode of the $\mathrm{D}$ band due to the frequency close to twice that of the D-band frequency. 


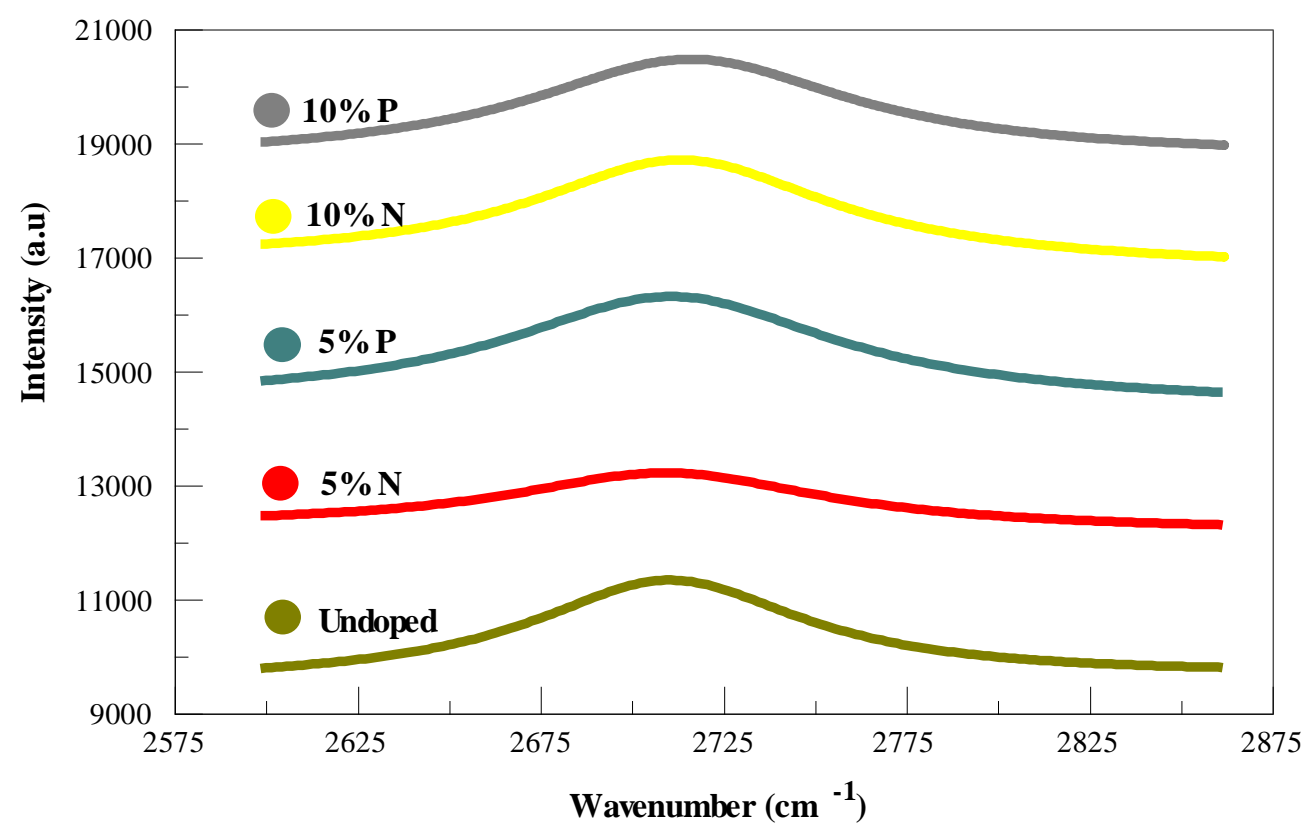

Figure 65: Raman Spectroscopy of as-synthesized MWCNTs in the region 2500-2900 $\mathrm{cm}^{-1}$

Table 11: G' bands from Raman spectroscopy of as-synthesized MWCNTs

\begin{tabular}{|c|c|c|c|c|c|}
\hline & Undoped & $5 \% \mathrm{P}$ & $10 \% \mathrm{P}$ & $5 \% \mathrm{~N}$ & $10 \% \mathrm{~N}$ \\
\hline $\mathrm{A}_{\mathrm{G}^{\prime}} \pm 3 \sigma$ (a.u) & $1740 \pm 40$ & $1560 \pm 60$ & $1740 \pm 30$ & $960 \pm 90$ & $800 \pm 50$ \\
\hline $\mathrm{B}_{\mathrm{G}^{\prime}} \pm 3 \sigma\left(\mathrm{x} 10^{4}\right)$ & $4.8 \pm 0.3$ & $3.3 \pm 0.4$ & $3.4 \pm 0.3$ & $3.7 \pm 0.2$ & $3.1 \pm 0.3$ \\
\hline $\mathrm{X}_{0 \mathrm{G}^{\prime}}\left(\mathrm{cm}^{-1}\right)$ & $2710 \pm 1$ & $2711 \pm 1$ & $2727 \pm 1$ & $2710 \pm 4$ & $2732 \pm 1$ \\
\hline
\end{tabular}

It has been well-known that the ratio of intensity of the $D$ over the $G$ band is an important characteristic to examine the defect concentration of CNTs [159-161]. A higher ratio corresponds to lower crystallinity of the tube due to increases of the D band intensity. Data from Table 10 show that this characteristic increases as a function of doping level. Significant increases were observed at doping concentrations $10 \%$ for both p-doped and n-doped samples (Figure 63). Compared to undoped CNTs, which has a ratio $0.54 \pm 0.04,10 \%$ n-doped and $10 \%$ p-doped counterparts provided relatively higher values of $0.68 \pm 0.05$ and $0.80 \pm 0.03$, respectively. Also, as can be seen, the full half width 
at maximum (FWHM $=1 / \mathrm{B})$ of the $\mathrm{D}$ band increases with increasing dopant concentration for both $\mathrm{n}$ - and p-doping. This value trend was expected since FWHM relates to a measure of the concentration of defects and crystallinity of the tubes [162]. Therefore, these characteristics are a confirmation of impurity insertion of as-synthesized doped CNT samples. Upon the introduction of triphenylborane and benzylamine as pand n-type doping sources, boron and nitrogen atoms are expected to be impurities, inserting into the lattice system of CNTs. However, actual quantitative doping concentration as well as type of lattice defects needs to be further addressed. Characterization techniques for such information should include X-ray photo electron (XPS) and scanning tunneling electron microscopy (STM).

Interestingly, also from Table 10, upon the introduction of defects into the lattice of CNTs by doping, the band frequencies of doped samples show noticeable shifts in both the D and G bands. Compared to undoped, the Raman frequency up-shift of the D band is more obvious in the case of $10 \%$ doping for both p-doped $\left(\sim 26 \mathrm{~cm}^{-1}\right)$ and n-doped $\left(\sim 24 \mathrm{~cm}^{-1}\right)$. Similarly, for the $\mathrm{G}$ band, the up-shift is $\sim 27 \mathrm{~cm}^{-1}$ and $\sim 31 \mathrm{~cm}^{-1}$ for $10 \% \mathrm{p}$ doped and 10\% n-doped, respectively. These observations are consistent with previous Raman studies of p-doped [163, 164] and n-doped [165, 166] CNTs. The up-shift frequency of the D band is a characteristic that indicates the appearance of new types of disorder in doped-CNTs relative to pure CNTs. Meanwhile, the up-shift frequency of G band could be attributed to a modification in the electronic structure of CNTs upon doping [165, 167, 168]. 
Another most pronounced difference between Raman spectra of undoped- and doped- CNTs was observed though the G' band (Figure 65). Data from our experiments show that the intensity, shape and position of this band are very sensitive to doping level. From Table 11, broader peak and red shift in frequency of G' bands are observed for both n-type and p-type doping. Additionally, there is a strong decrease in intensity ratio between the $G$ and $G^{\prime}$ bands (Figure 66). This observation is consistent with previous observations in graphene [169] and CNT [165].
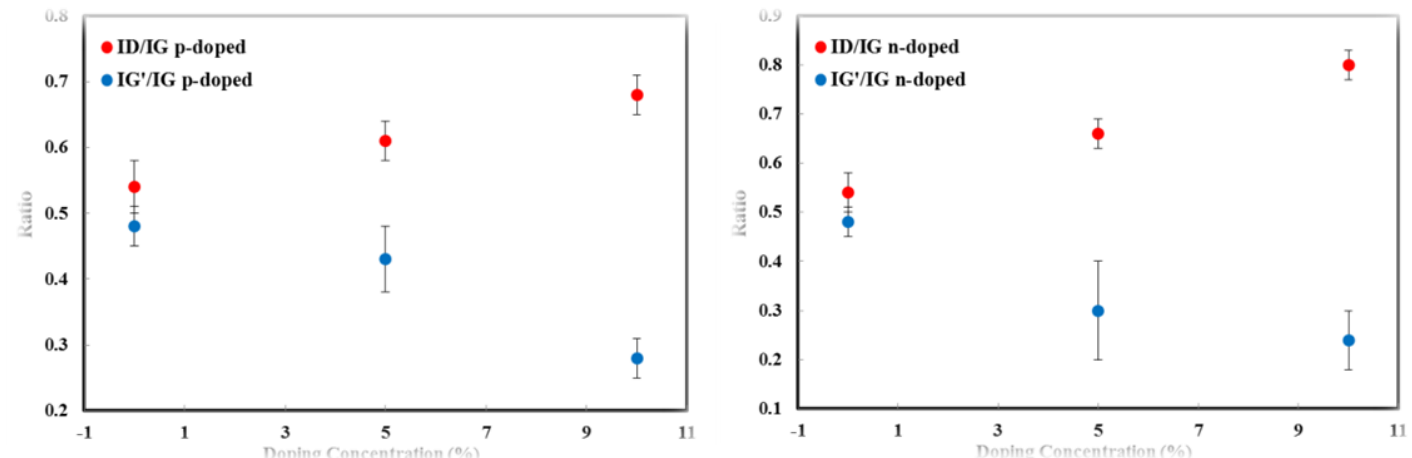

Figure 66: The band intensity ratios of $\mathrm{D} / \mathrm{G}$ and $\mathrm{G}$ '/G of p-doped CNTs (left) and n-doped CNTs (right).

\subsubsection{Pd nanoparticle attached MWCNTs for hydrogen sensor}

\section{A. Attachment of Pd NPs on commercial CNTs}

Commercial CNTs used in this experiment had average diameters from $20-40 \mathrm{~nm}$ and length varying from 5-10 $\mu \mathrm{m}$ (Figure 67A). The in-situ Pd nanoparticle decorated CNTs were synthesized according to section 2.1.2.3. An SEM image of CNT with attached Pd nanoparticles on commercial CNTs is showed in Figure 67B. It shows 5-10 nm size Pd NPs are attached to CNTs with different densities. 


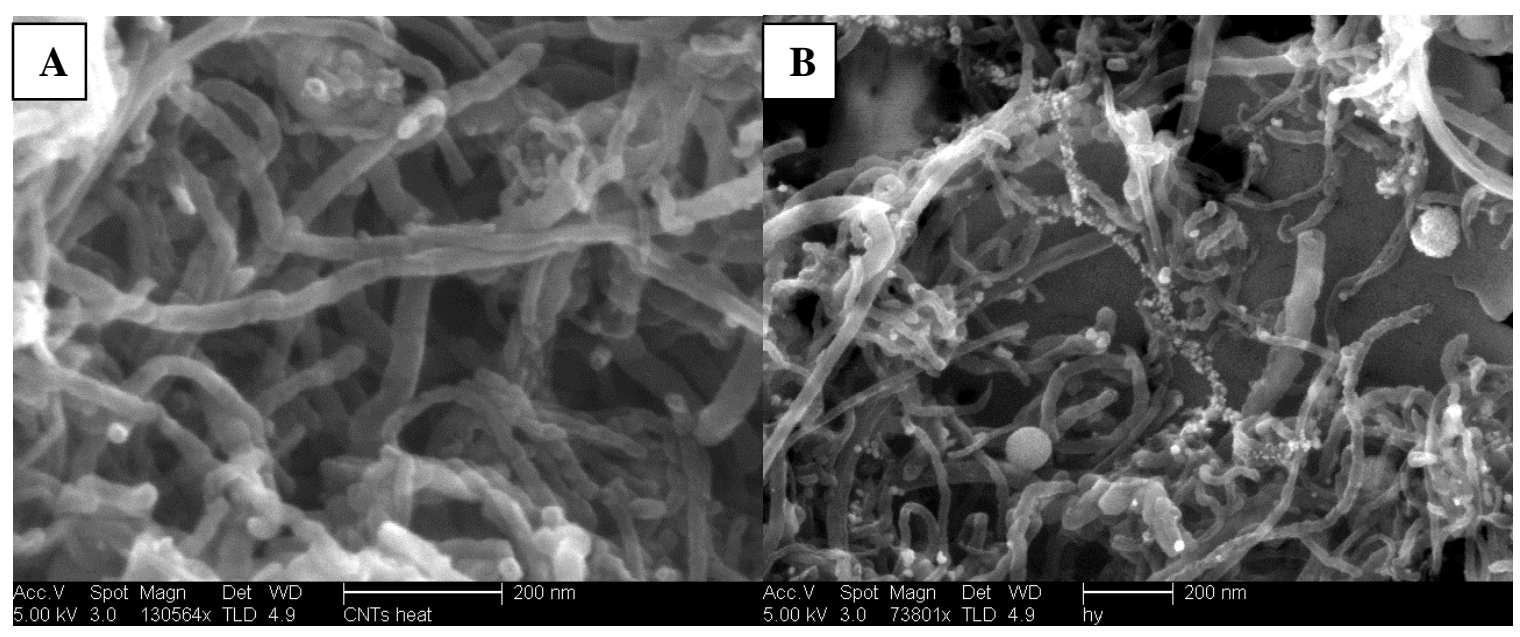

Figure 67: SEM images of commercial CNTs and palladium nanoparticle decorated CNTs.

The mechanism of $\mathrm{Pd}$ nanoparticle decoration is presented in Figure 68. In general, the attachment involves two steps including wetting the surface of CNTs by SDS during the sonication and the reduction of palladium acetate to Pd nanoparticles during the reflux process. SDS plays a role as a surface wetting agent, and 1-dodecanol generated from thermal conversion of SDS acts as a reducing agent. Pd-CNTs are well dispersed in aqueous medium by stabilizer components, constituted mainly of 1dodecanoic acid, 1-dodecanol and unconverted SDS.

$$
\mathrm{CH}_{3}\left(\mathrm{CH}_{2}\right)_{11} \mathrm{OSO}_{3} \mathrm{Na} \underset{\text { Conversion }}{\stackrel{\text { Thermal }}{\longrightarrow}} \mathrm{CH}_{3}\left(\mathrm{CH}_{2}\right)_{11} \mathrm{OH} \stackrel{\mathrm{Pd}^{2+}}{\longrightarrow} \mathrm{CH}_{3}\left(\mathrm{CH}_{2}\right)_{10} \mathrm{COOH}
$$




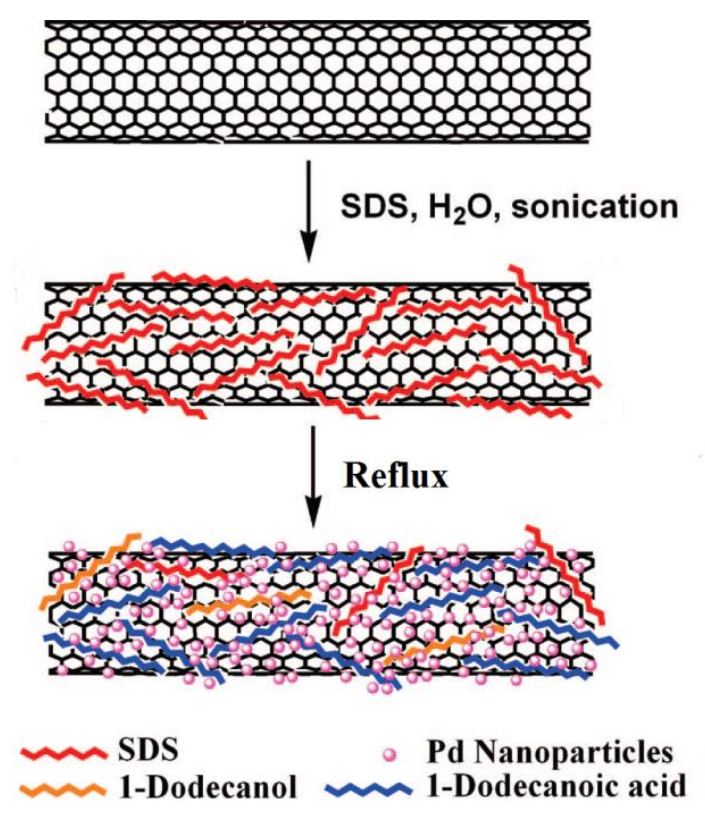

Figure 68: Illustrative concept for the Decoration of CNTs with Pd Nanoparticles. This figure is adapted and modified from Ref [31].

B. Attachment of Pd nanoparticles on as-synthesized CNTs

CNTs synthesized by method C using cobaltocene (Figures 69A and 69B) were employed for the attachment of Pd nanoparticles. After the synthesis, hybrid structure of Pd NPs and CNTs is shown in Figures 69C and 69D. As observed, much higher density of Pd nanoparticle attachment compared to its counterpart using commercial CNTs. The walls of CNT are decorated with different sizes of Pd nanoparticles, shown in TEM images (Figure 70). This could be the result of an as-synthesized CNT characteristic, which have more defective sites for the attachment reaction. Higher density of PdNanoparticles turns out to be a better hybrid material for $\mathrm{H}_{2}$ detection as shown later. 


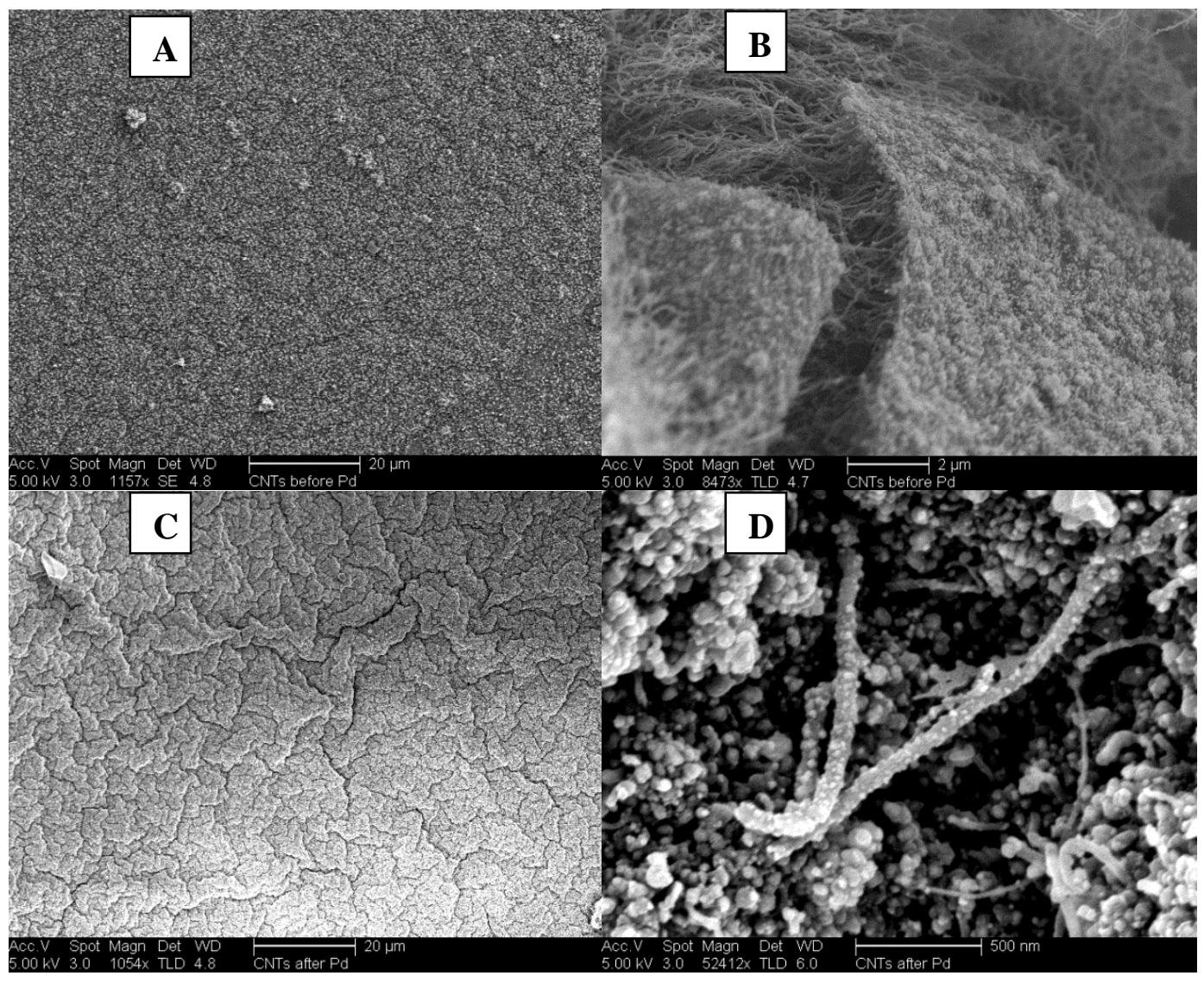

Figure 69: SEM of thin film of CNTs deposited on the wall of glass tube before (A and B) and after (C and D) the attachment of Pd nanoparticles.

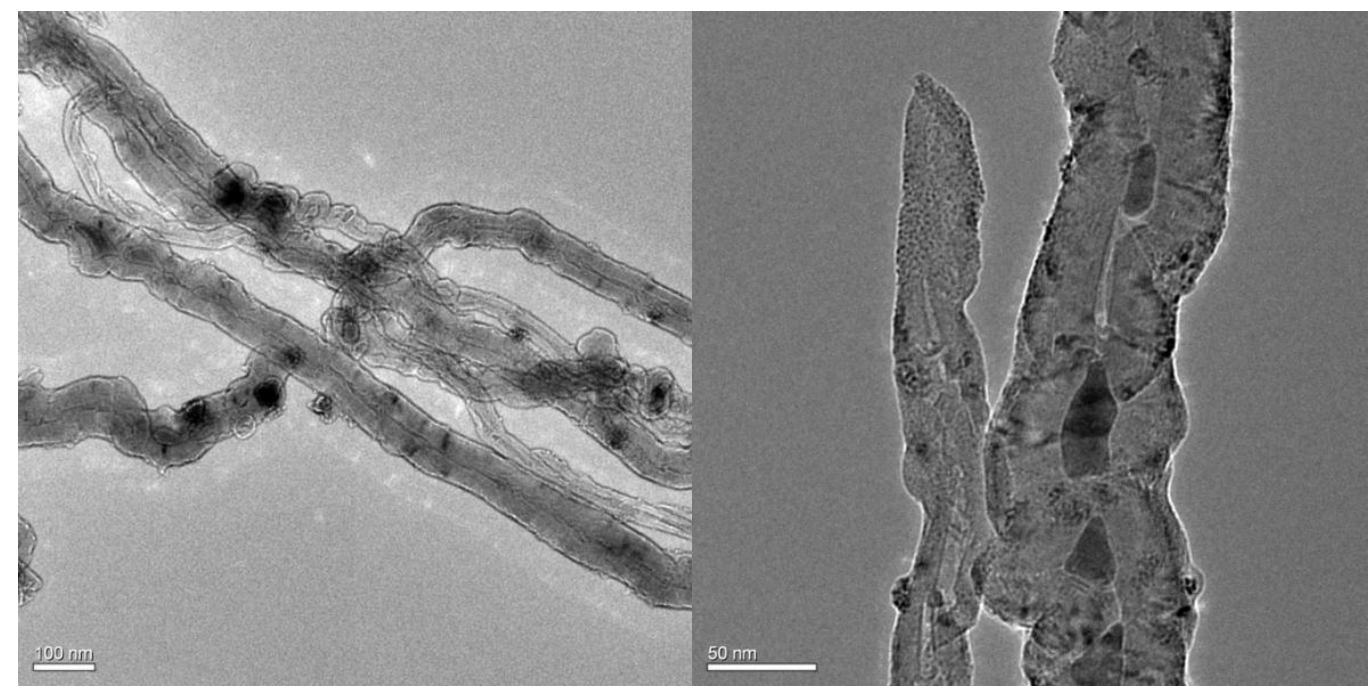

Figure 70: Low and high magnification TEM images of as-synthesized CNTs decorated with Pd nanoparticles 


\subsection{Sensing Application: Hydrogen Gas Sensor Based on Pd NPs Attached MWCNTs}

In this section, the sensing performance and mechanism of sensors, using a hybrid structure of Pd nanoparticle attached CNTs, toward hydrogen gas is presented. Similar to the $\mathrm{Cl}_{2}$ gas sensor, the study is based on measurements of direct current conductivity to establish the sensing model.

\subsubsection{Sensing Performance}

Initial testing and understanding about the response of Pd-CNTs hybrid structure toward hydrogen was carried out using commercial MWCNTs. The response of sensor fabricated from Pd nanoparticles decorating commercial CNTs is showed in Figure 71. It was observed that the exposure of the as-constructed sensor to continuous flow of $4 \%$ of $\mathrm{H}_{2}$ diluted in nitrogen resulted in a slow increase in sensor resistance. It took about 15 minutes for the sensor to achieve the maximum resistance and about 1.5 hours to recover upon refreshing with air at room temperature.

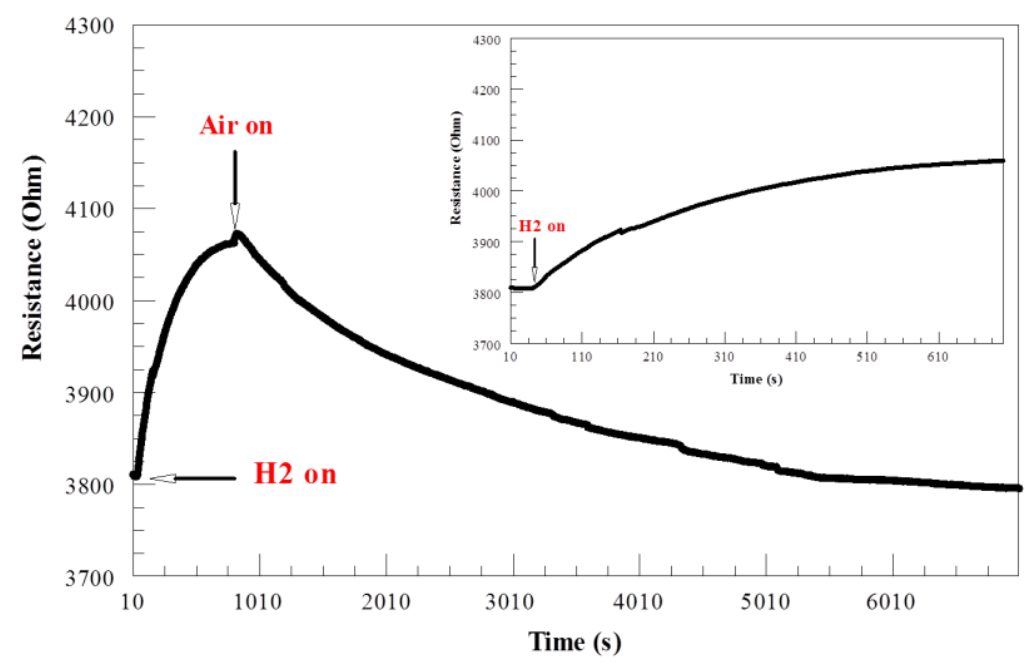

Figure 71: Response of testing sensor upon the exposure of $4 \% \mathrm{H}_{2}$ with the inset showing the initial stage of $\mathrm{H}_{2}$ exposure with the flow rate of $1 \mathrm{~L} /$ minute 
Previous studies have showed that attachment of $\mathrm{Pd}$ nanoparticles impart remarkable sensitivity on the electrical properties of nanotubes toward molecular hydrogen. With its ability to split hydrogen molecules into hydrogen atoms, Pd nanoparticles play a role as a catalyst in the interaction between $\mathrm{H}_{2}$ molecules and nanotubes [29, 170]. Specifically, hydrogen molecules split into atomic hydrogen spillover from Pd nanoparticles to CNTs. This is followed by electron transfer to CNT surface and changes in carrier density leading to changes in the resistance $(\mathrm{R}-1 / \mathrm{n}$, where $\mathrm{n}$ is the carrier density) of the CNT. Therefore, as can be seen in Figure 70, the slow response in this case could be a result of a slow electrons transfer rate to nanotubes, caused by low density of Pd nanoparticles attached on MWCNTs. Such response also makes the asfabricated sensor impractical, and thus a higher density of attached Pd nanoparticles is required to improve sensor performance.

Replacing commercial CNTs by as-synthesized CNTs, a similar response characteristic was obtained. The sensor resistance increased upon the exposure to $\mathrm{H}_{2}$ and quickly reverses when hydrogen gas is turned off, followed by an introduction of dry air into the testing chamber. However, a much faster response time as well as a higher detection limit was achieved in this case. Response and recovery of the sensor were orders of magnitude faster than what was observed from commercial CNTs. The result of $\mathrm{H}_{2}$ detection by CNTs, synthesized by the sealed glass tube growth method $\mathrm{C}$ following attachment of Pd nanoparticles, is showed in Figure 72. The investigation was carried by exposing the sensor with different $\mathrm{H}_{2}$ concentrations diluted in $\mathrm{N}_{2}$ from $10 \mathrm{ppm}$ to $4 \%$. 


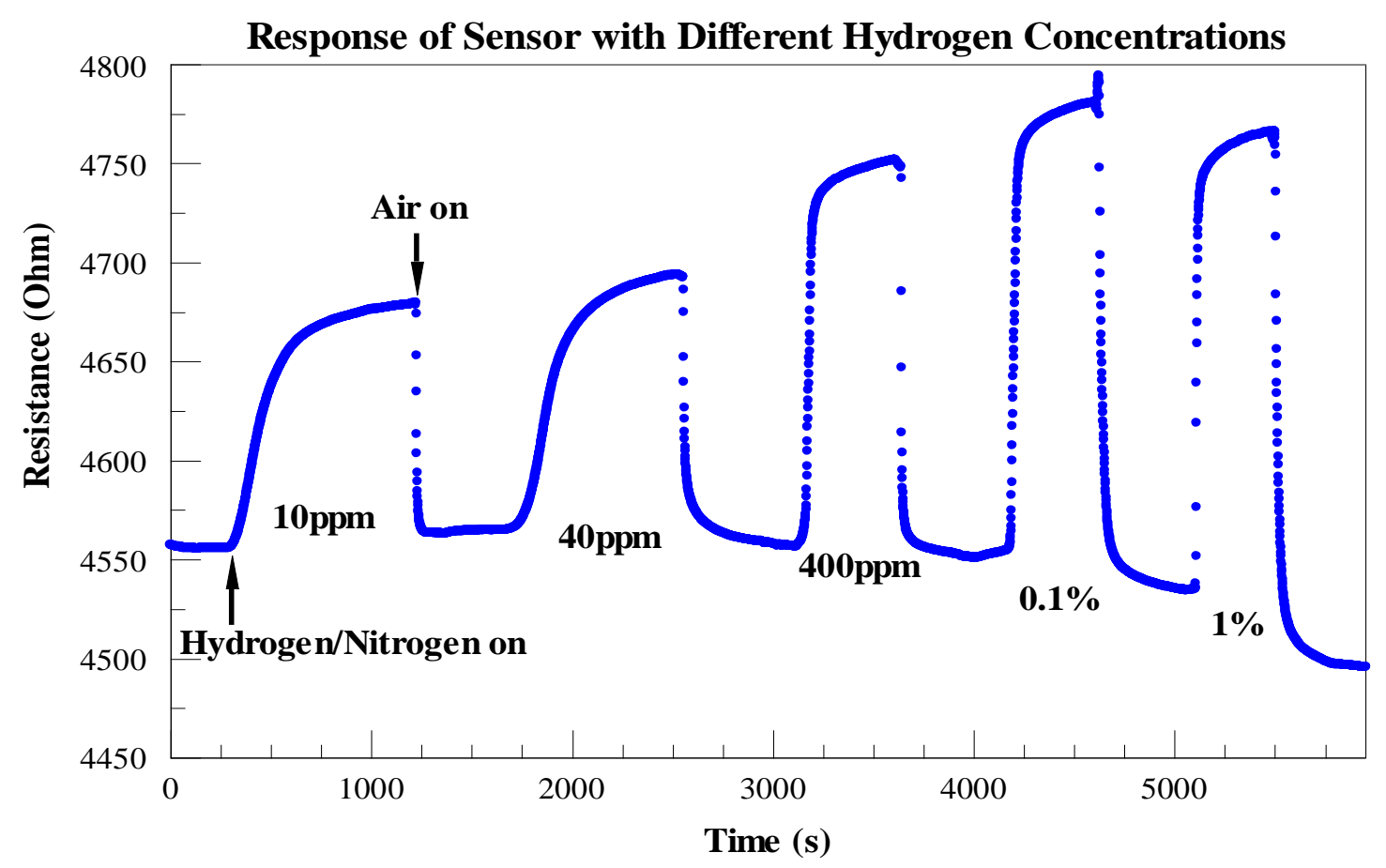

Figure 72: Response of Pd nanoparticles attached as-synthesized CNTs sensor to different $\mathrm{H}_{2}$ concentrations from $10 \mathrm{ppm}$ to $4 \%(40,000 \mathrm{ppm})$.

Sensor resistance changed about $200 \Omega$ during the response to such a range of $\mathrm{H}_{2}$ concentrations. During exposures to $10 \mathrm{ppm}_{2}$, the sensor response time was $\sim 10$ minutes. This time became shorter with higher $\mathrm{H}_{2}$ concentration. Full sensor recovery time was much quicker than response time, in the range of 30-50 seconds. This result clearly shows that the sensor composed of Pd NPs decorated as-synthesized CNTs provides much better sensor performance compared to its counterpart made using commercial MWCNTs.

To understand the sensor response kinetics, the sensor response curves were fitted to a third order polynomial. Figure 73 shows an example of fitting at $10 \mathrm{ppm} \mathrm{H}_{2}$ concentration.

$$
R=a_{3} t^{3}+a_{2} t^{2}+a_{1} t+R_{0}
$$


Where:

$R$ is the sensor resistance response during the exposure $(\Omega)$

$t$ is the exposure time

$a_{1}, a_{2}, a_{3}$ are fitting constants

$R_{0}$ is the sensor resistance baseline

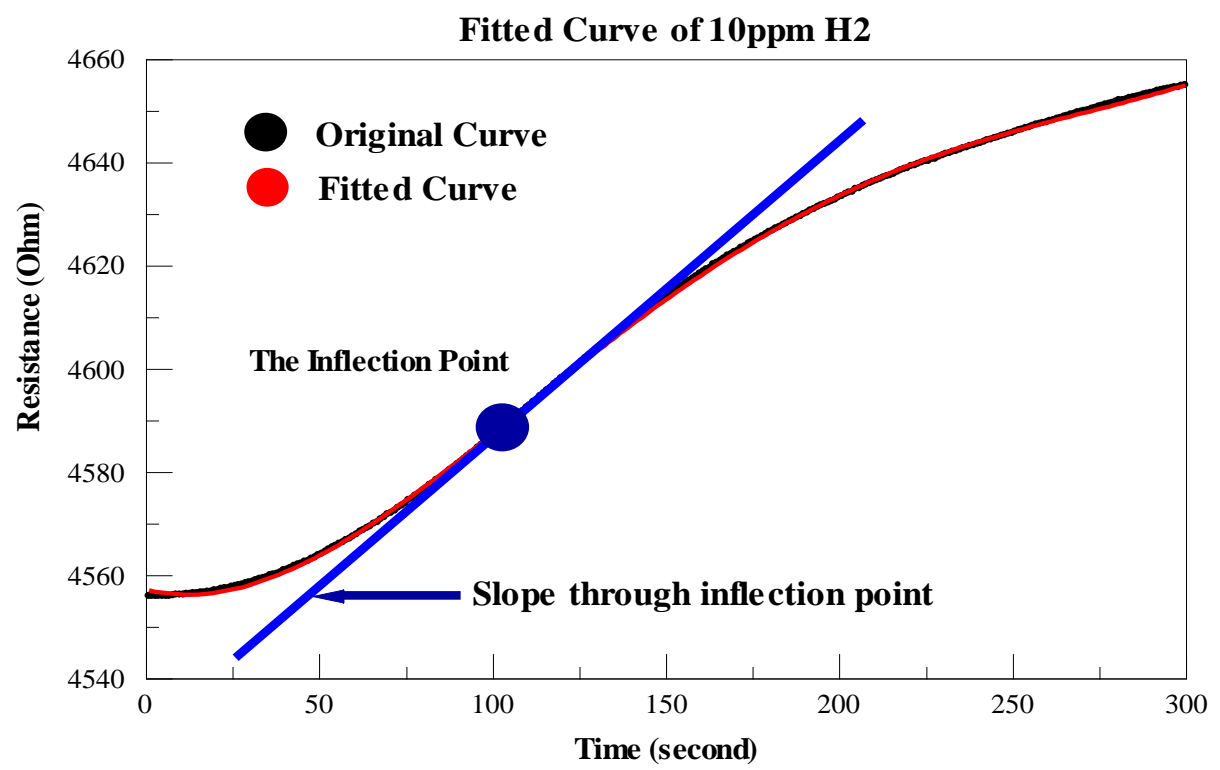

Figure 73: An example of fitted curve to $10 \mathrm{ppm}_{2}$ concentration following (eq. 4.11)

Instead of using conventional response (the ratio of the change in sensor resistance on exposure to target gas versus original sensor resistance in air), the slopes at inflection point of fitting curves were employed to investigate the relationship between sensor response and exposed hydrogen concentration. These slopes were plotted as a function of $\mathrm{H}_{2}$ concentration with the data shown in Figure 74. 


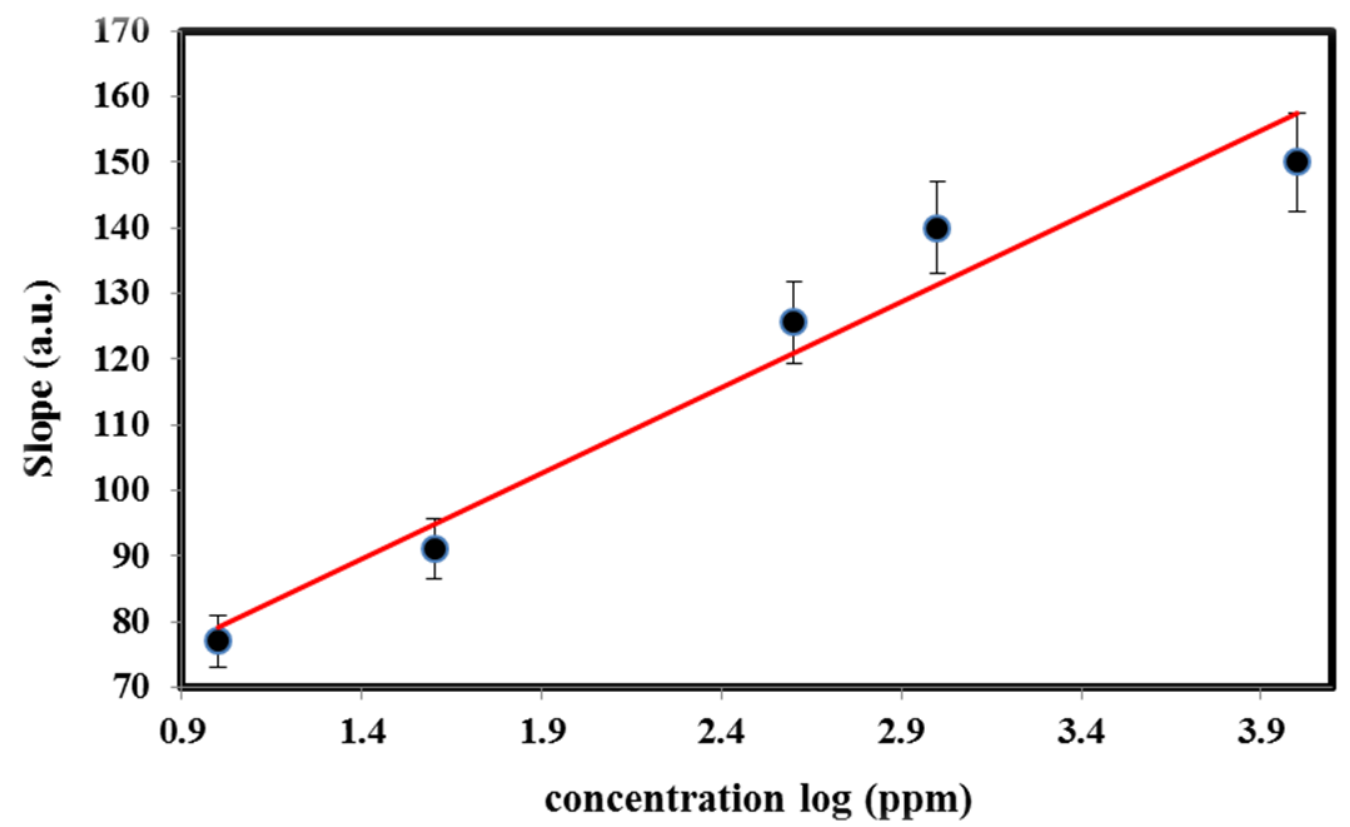

Figure 74: Slope of the response curve as a function of $\mathrm{H}_{2}$ concentrations with slope $=26.2 \log \left[\mathrm{H}_{2}\right]+53$. Small black circles represent raw data, and the red line corresponds to fitted data as a linear line.

Figure 74 shows that the slope of the sensor response curve increases approximately linearly with the log of the hydrogen concentration $(\mathrm{ppm})$, slope $=26.2$. Since the slope is related to the sensor response time, the increase of slope value is the result of faster sensor response. This has opened a new way to correlate sensor response and target gas concentration, in the case of the magnitude of conventional sensor response (i.e change in resistance) does not vary significantly upon exposure. However, due to logarithmic concentration, dependence of slope makes differentiation of two close exposure concentrations could be difficult. Comparison with commercial CNTs and CNTs synthesized in this study indicate that Pd nanoparticles density is proportional to sensor response time, and thus the slope of response curve. This also could be a factor leading to better sensor performance as observed. 
An interesting phenomenon was observed at higher concentrations of $\mathrm{H}_{2}$ in Figure 75. Specifically, the sensor shows two different response characteristics corresponding to different levels of $\mathrm{H}_{2}$ concentration exposure (above and below $1 \%$ hydrogen concentration). At $1 \%$ of $\mathrm{H}_{2}$ concentration, normal sensing behavior was similar to low $\mathrm{H}_{2}$ concentrations. Continuous exposures to higher than $1 \% \mathrm{H}_{2}$ in $\mathrm{N}_{2}$ showed that the sensor resistance still varies in the range of $200 \Omega$, but its initial sharp rise is followed by an abrupt decrease and followed by a stabilization of resistance during the exposure process. During the recovery process by refreshing with air, unusual behavior was also observed. The sensor resistance increased quickly to a certain level of resistance and then went back down to almost the original resistance. Further analysis to understand this behavior will be devoted in following sections.

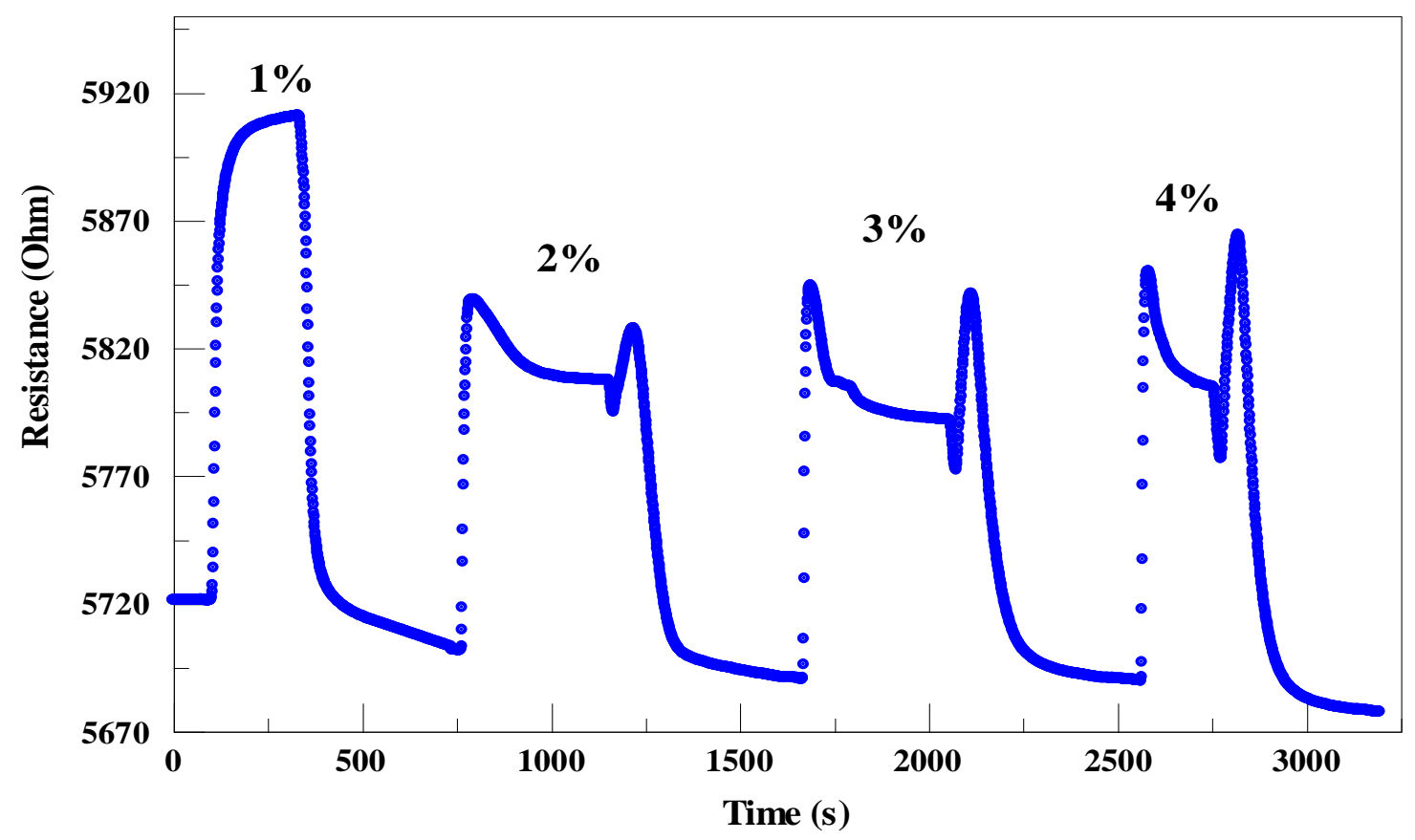

Figure 75: Abnormal behaviors of sensor response and recovery at high $\mathrm{H}_{2}$ concentrations. 


\subsubsection{Understanding Sensing Mechanism:}

A. General sensing mechanism

Since the size of the nanotubes is greater than $7 \mathrm{~nm}$, synthesized nanotubes are metallic. The CNT Fermi level as well as the reduction potential of $\mathrm{H}_{2}$ and $\mathrm{O}_{2}$ are presented in Figure 76. The underlying mechanism of sensing is attributed to the interaction between target gas $\left(\mathrm{H}_{2}\right)$, catalysts (Pd nanoparticles), and nanotubes with a model illustrated in Figure 77. It is well-established that Pd nanoparticles catalytically activate the dissociation of molecular hydrogen [170, 171], and CNTs are p-type semiconductor material in ambient condition due their oxidation (see Figure 76) by atmospheric oxygen [172-174]. Upon adsorption on the surface of Pd nanoparticles, $\mathrm{H}_{2}$ molecules undergo dissociative chemisorption to split into atomic hydrogen atoms. The resulting atomic hydrogen atoms could either dissolve into Pd with high solubility or diffuse away from receptor sites of Pd and move onto the surface of the CNTs. Since the dissolution of atomized hydrogen into Pd lowers the work function of Pd, [28, 175], spilled-over hydrogen atoms donate electrons to nanotubes [171] through receptor sites or defects of CNTs thereby annihilating holes. This leads to the decrease in charge carrier concentration. 


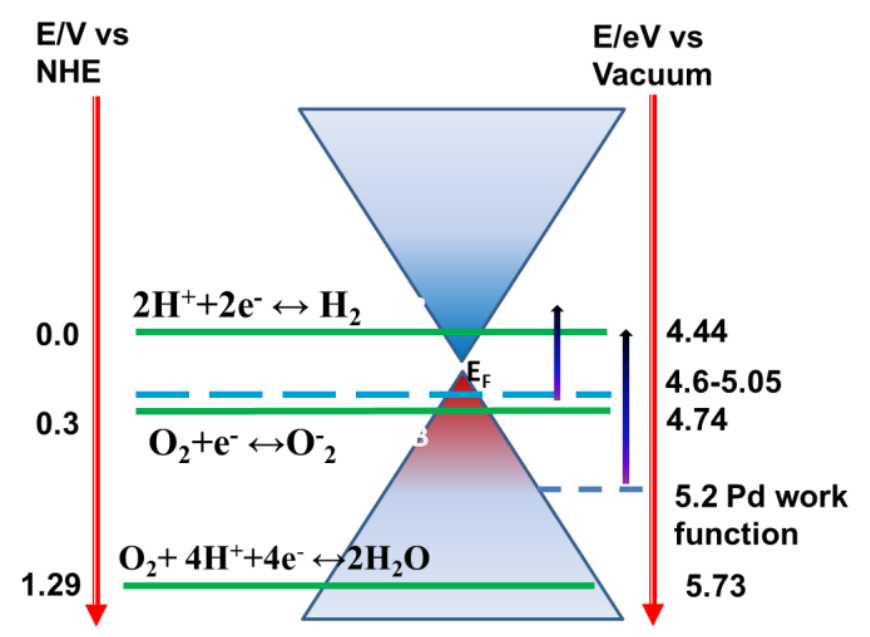

Figure 76: Electrochemical energy level diagram of hydrogen, palladium and oxygen in relation to the Fermi level of CNTs (dashed blue line). Dissolution of hydrogen reduces the Pd work function shifting it upwards as indicated. Donation of electron from the spilled hydrogen atom changes the Fermi level in the same direction as indicated by arrows, reducing the hole concentration in the p-type CNT. Green horizontal lines indicate electrochemical energy states of the analyte where electron/hole transfer could take place

Donation of an electron the from hydrogen atom results in formation of protons on the CNT surface which repels the majority carrier, holes. Consequently, a depletion layer at the CNT interface is established. Reduction in carrier concentration and formation of depletion layer lead to sensor resistance increase.

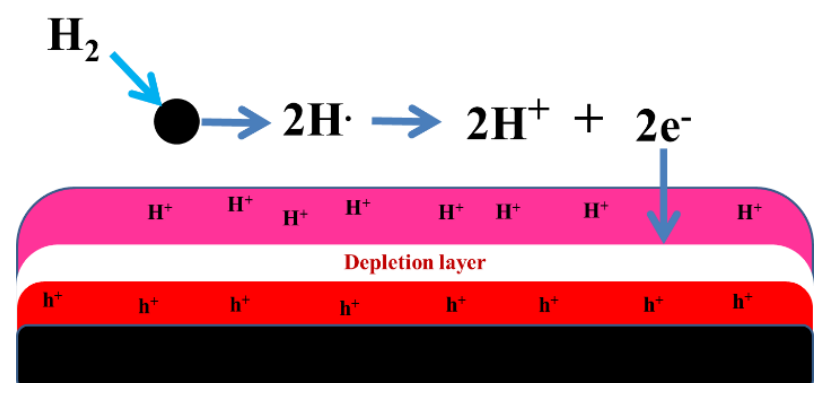

Figure 77: Illustration of the response mechanism of Pd Nps decorated CNTs upon the exposure with $\mathrm{H}_{2}$.

In summary, the kineticsz of this overall process can be illustrated as:

$$
\frac{1}{2} H_{2(\text { surf })} \stackrel{k_{1}}{\rightarrow} H \cdot \stackrel{k_{2}}{\rightarrow} H_{\text {surf }}^{+}+e
$$


Therefore, the fitting of each sensor response curve to show sensor resistance as a function of exposure time is based on following equation (eq. 4.12) which reflects decreased hole concentration in CNT as resulting from successive two kinetic steps:

$$
R=R_{0}\left(1-\frac{k_{1}}{k_{1}-k_{2}}\left(e^{-k_{1} t}-e^{-k_{2} t}\right)-e^{-k_{1} t}\right)+C
$$

Where:

$\mathrm{k}_{1}$ and $\mathrm{k}_{2}$ are kinetic constants corresponding to $\mathrm{H}_{2}$ molecular dissociation by $\mathrm{Pd}$, and electrons transferred to nanotube, respectively.

$\mathrm{R}_{0}$ is the fitting constant

$\mathrm{C}$ is the fitting resistance baseline

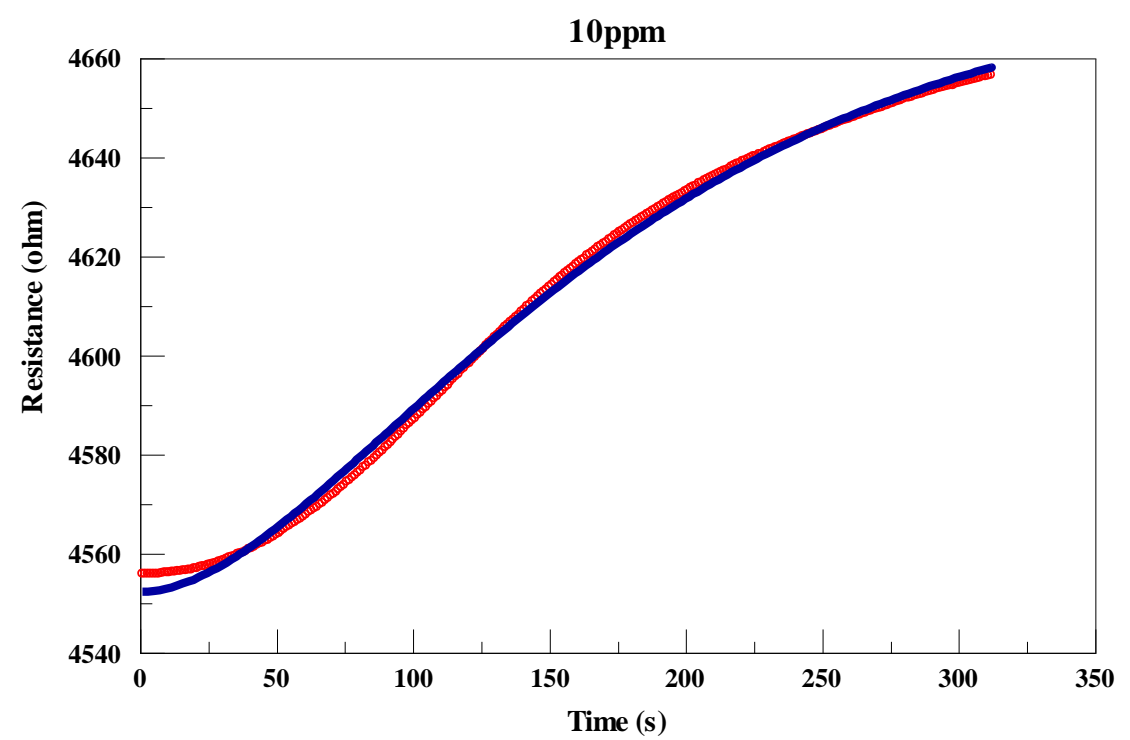

Figure 78: Sensor response at $10 \mathrm{ppm} \mathrm{H}_{2}$ concentration: sensor resistance changes as a function of exposure time. Red line and blue line correspond to raw and fitted data, respectively.

Table 12: Summary kinetic constants from fitted response curves

\begin{tabular}{|l|l|l|l|l|l|}
\hline & $10 \mathrm{ppm}$ & $40 \mathrm{ppm}$ & $400 \mathrm{ppm}$ & $1000 \mathrm{ppm}$ & $10000 \mathrm{ppm}$ \\
\hline $\mathrm{k}_{1} \times 10^{2}$ & $1.1 \pm 0.1$ & $0.9 \pm 0.1$ & $5.4 \pm 1$ & $61 \pm 5$ & $16000 \pm 100$ \\
\hline $\mathrm{k}_{2} \times 10^{2}$ & $1.1 \pm 0.1$ & $0.9 \pm 0.1$ & $4.2 \pm 1$ & $9 \pm 2$ & $16.2 \pm 3$ \\
\hline $\mathrm{R}_{0}$ & $124 \pm 10$ & $180 \pm 41$ & $283 \pm 30$ & $281 \pm 30$ & $285 \pm 50$ \\
\hline
\end{tabular}


The investigation and curve fitting were based on 5 different concentrations with an example shown in Figure 78, corresponding to $10 \mathrm{ppm}_{2}$ exposure. As can be seen, both kinetic constants increase nonlinearly with the increase of $\mathrm{H}_{2}$ concentration indicating that the other kinetic steps need to be included to properly fit the data. At very low $\mathrm{H}_{2}$ concentrations such as $10 \mathrm{ppm}$ and $40 \mathrm{ppm}$, these constants seem to be stable at a value around $\sim 10^{-2} \mathrm{~s}^{-1}$. They rapidly increase at high $\mathrm{H}_{2}$ concentrations, especially at $1 \%$, at which the sensor starts showing abnormal response and recovery behaviors. It was observed that the values of $k_{2}$ and $\log$ of $k_{1}$ increased exponentially as a function of $\log$ of exposed $\mathrm{H}_{2}$ concentration. These relationships are shown in Figure 79.
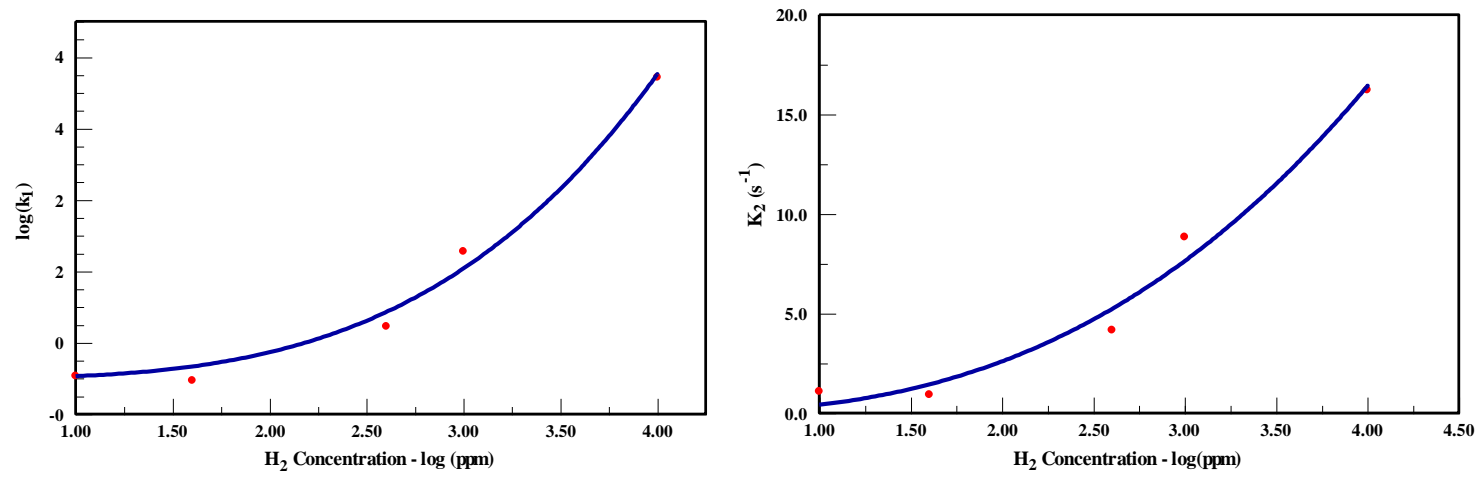

Figure 79: $\mathrm{H}_{2}$ molecular dissociation constant (left) and electrons transferred to nanotube constant (right) change as a function of $\mathrm{H}_{2}$ concentration: $\log \mathrm{k}_{1}=0.03(\log \mathrm{C})^{3.5}$ and $\mathrm{k}_{2}=0.4(\log \mathrm{C})^{2.7}$ where $\mathrm{C}$ is $\mathrm{H}_{2}$ concentration (ppm).

Reversibility of sensor resistance during the recovery process with air would involve the effect of $\mathrm{O}_{2}$. Ultimately $\mathrm{O}_{2}{ }^{-}$species on the CNT surface can be regenerated by removing hydrogen from the surface. Hydrogen atoms, including dissolved in and spilled over Pd nanoparticles, could combine with oxygen in air along with a withdrawing electron from the conduction band of CNTs, departing the hybrid system in the form of volatile hydrogen di-oxide vapor. Also a hydrogen free surface can 
host $\mathrm{O}_{2}$ and further depletes electrons from the VB as showed in Figure 76. As a result, the p-type nanotube interface is recovered, and the sensor resistance goes back to the original resistance level.

This process can be kinetically represented as:

$$
H_{\text {surf }}^{+}+\mathrm{O}_{2}+e \stackrel{k_{3}}{\rightarrow} \mathrm{HO}_{2} ; \mathrm{O}_{2}+e \stackrel{k_{3 \prime}}{\rightarrow} \mathrm{O}_{2}^{-}
$$

Thus, each recovery curve can be fitted according to equation below:

$$
R=R_{0} e^{-k_{3} t}+C
$$

Where:

$R$ is the sensor resistance

$k_{3}$ is the desorption kinetic constant.

$k_{3}$, is rate of electron abstraction from hydrogen depleted CNTs

$R_{0}$ is the fitting constant

$C$ is the fitting resistance baseline

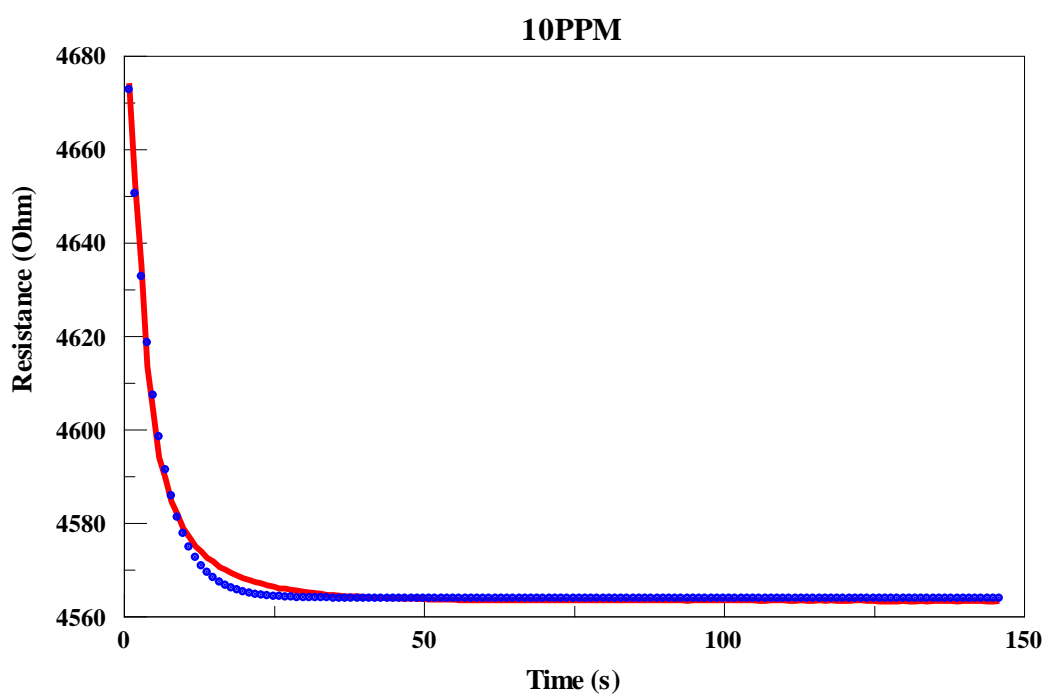

Figure 80: Sensor recovery after $10 \mathrm{ppm}_{2}$ exposure: sensor resistance change as a function of recovery time. Red line and blue line correspond to raw and fitted data, respectively. 
Table 13: Summary of kinetic constants from fitted recovery curves

\begin{tabular}{|c|c|c|c|c|c|}
\hline & $10 \mathrm{ppm}$ & $40 \mathrm{ppm}$ & $400 \mathrm{ppm}$ & $1000 \mathrm{ppm}$ & $10000 \mathrm{ppm}$ \\
\hline $\mathrm{k}_{3}$ & $0.23 \pm 0.01$ & $0.11 \pm 0.01$ & $0.17 \pm 0.01$ & $0.08 \pm 0.01$ & $0.08 \pm 0.01$ \\
\hline $\mathrm{R}_{0}$ & $136 \pm 4$ & $127 \pm 3$ & $248 \pm 4$ & $208 \pm 5$ & $210 \pm 5$ \\
\hline
\end{tabular}

From the data, it has indicated that the desorption kinetic constant during the recovery process has a consistent value $\sim 0.1 \mathrm{~s}^{-1}$ and does not depend on hydrogen exposure concentration.

Additionally, as can be seen on Figure 75, the sensor resistance baseline tends to drift to lower resistance after many cycles of exposure and recovery processes. It is believed that this behavior relates to the restoration of $-\mathrm{CH}_{2}$ in $\mathrm{CNT}$ structure. As showed in the morphological characterizations, as-synthesized CNTs using this synthetic method are highly defective and heavily oxygenated. Defects could be derived from mostly oxygen functional groups including $\mathrm{OH}-$ and $-\mathrm{COOH}$ decorating $\mathrm{CNT}$ surface and edges [176]. During the exposure, $\mathrm{H}_{2}$ molecules are dissociated by $\mathrm{Pd}$ particles to atomic hydrogen. Via spillover and surface diffusion [177], these species could remove the defective groups in the form of water vapor and $\mathrm{CO}_{2}$ [178] as well as subsequently restore $-\mathrm{CH}_{2}$ composition. Through many reduction (exposure) and oxidation (recovery) cycles, higher degree of crystallinity CNTs is recovered and leads to defect-free CNTs, decreasing the overall sensor resistance.

B. Sensing mechanism of abnormal response behavior at high concentration exposure:

In order to understand better the abnormal behavior of sensor resistance change, experiment involving a single cycle of exposure to high concentration $\mathrm{H}_{2}$ and recovery in dry air was carried out as shown in Figure 81. 


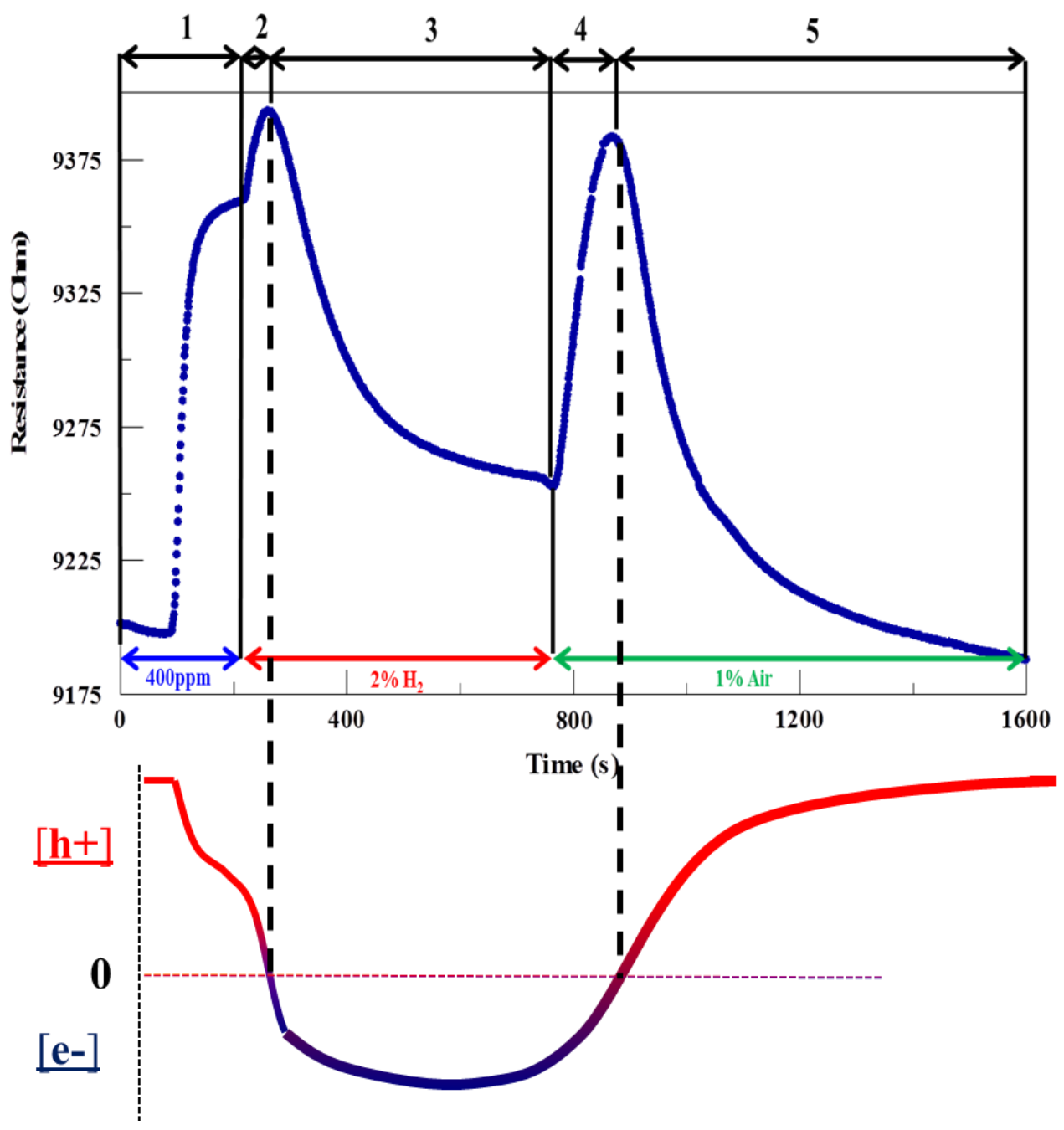

Figure 81: a) 5 stages of charge carrier inversion process b) Time dependence of excess hole and electron concentration in CNT. Zero on the vertical axis indicates intrinsic CNT. Red and Purple line colors indicate n-type and p-type CNT, respectively.

The experimental data was collected by exposing the sensor to two different hydrogen concentrations and finally refreshing the sensor with a flow of $1 \%$ of air in nitrogen. The entire exposure and regeneration cycle of the sensor is illustrated through 5 stages. Initially, low concentration of $\mathrm{H}_{2} 400 \mathrm{ppm}$ was used to bring and stabilize the sensor resistivity to maximum response resistance (stage 1). It was then exposed to a high 
concentration of $2 \% \mathrm{H}_{2}$. Such a concentration initially increased sensor resistance and was followed by an abnormal resistance decrease response behavior as mentioned above. The sensor resistance, after reaching the maximum value (stage 2), decreased thereafter to lower level of resistivity and eventually stabilized (stage 3). During the recovery process, $1 \%$ of air in $\mathrm{N}_{2}$ was used instead of air to reduce the rate of recovery reaction. With such refreshing, behavior of this process can be more readily observable. The sensor resistance went back up to almost the same level of maximum resistance (stage 4) followed by a resistance decrease as in normal recovery behavior (stage 5).

The observed abnormal behavior could be rationalized in terms of charge carrier inversion. It assumes that CNTs are converted from p-type to n-type and then back to original the p-type carriers during a cycle of exposure to $\mathrm{H}_{2}$ and recovery with $\mathrm{O}_{2}$ in air. The scheme to explain 5 stages of this process is illustrated in Figure 82. 


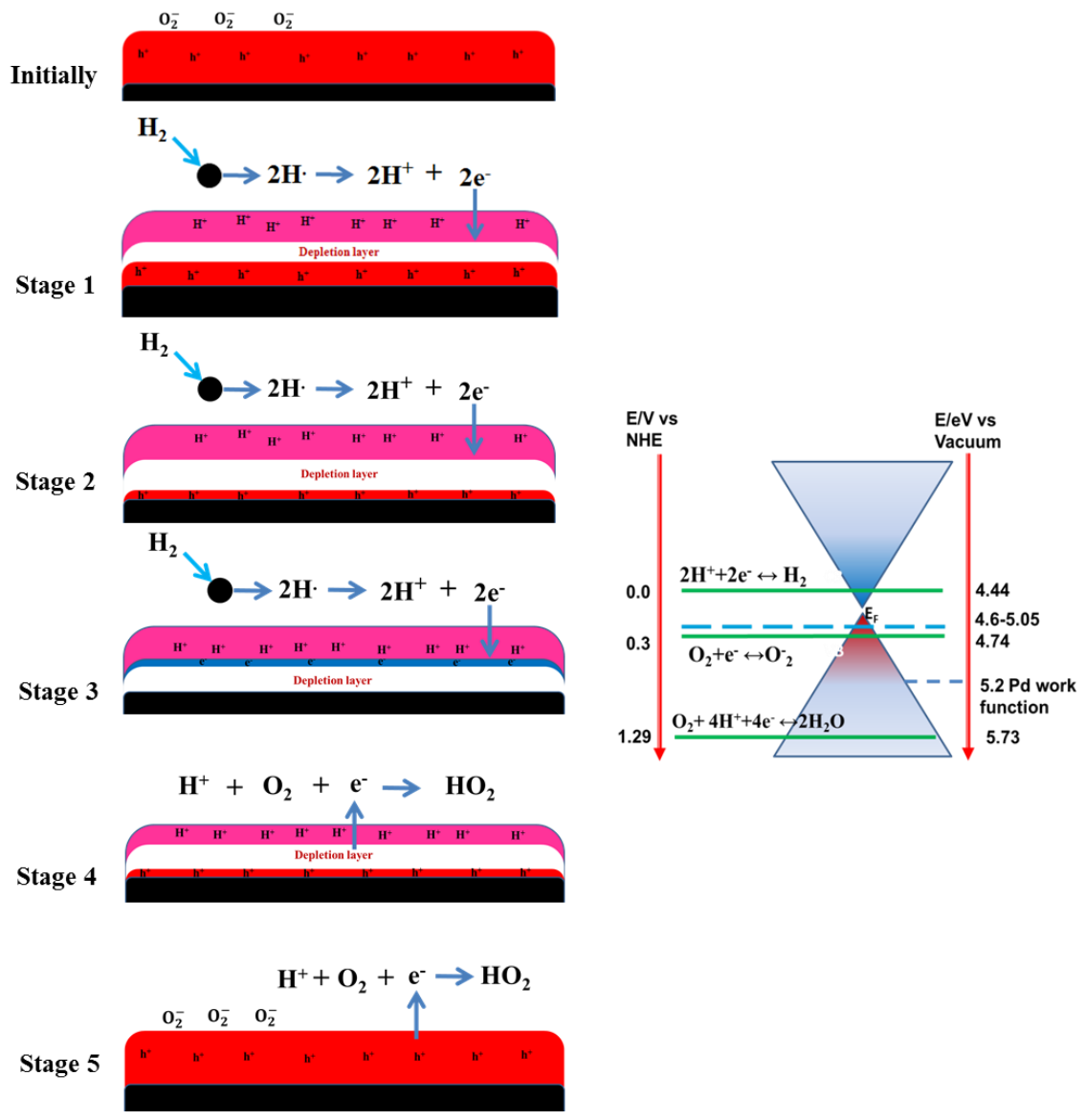

Figure 82: Mechanism of 5 stages of charge carrier inversion process (left) and electronic band diagram of sensing process (right)

Initially, in an ambient environment exposed to air, MWCNTs are p-type semiconductors with respect to the electronic property of the outermost layer. During the first stage of exposure of low $\mathrm{H}_{2}$ concentration (stage 1), the reduction of CNT by hydrogen brings the hole concentration down (Figure 82 left). Surface bound protons repel the majority carriers away generating a depletion layer. In this process the Fermi level of CNT begins to shift from valance band towards the conduction band due to accumulation of electrons in the VB. This layer is built up during high $\mathrm{H}_{2}$ concentration exposure in stage 2 due to electron transfer from split hydrogen atoms to CNT the interface to neutralize p-type charge carriers. A maximum resistance is expected when 
the excess hole concentration is quenched, corresponding to the shift of CNT Fermi level between VB and CB. Ultimately in stage 3, the value of electron concentration increases along with the increase of high $\mathrm{H}_{2}$ concentration exposure time. As a result, the Fermi level is shifted close to $\mathrm{CB}$, making CNT n-type. Also, in this stage, the sensor resistance stabilizes at the lowest level resistivity corresponding to saturated electron concentration on surface of nanotube. Ultimately, the Fermi level should lines up with hydrogen redox potential showed in Figure 82 (right).

The reversal of charge carrier inversion occurs in stage 4 during the recovery process. During this stage, $\mathrm{O}_{2}$ in air react with $\mathrm{H}^{+}$on the nanotube surface along with withdrawing electrons from CNT to form volatile dihydrogenoxide molecules. This process reduces and eliminates the excess electron concentration built up during stage 3 . The maximum resistance value in this stage is consistent with its counterpart in the stage 2 derived from maximum depletion layer and minimum charge carrier concentration. At this point the Fermi level should be at the center of band gap. When air continued flowing into the sensor in stage $5, \mathrm{O}_{2}$ physisorbs on the nanotube wall as well as diffuses into the edge, inducing p-type doping as discussed before. The sensor resistance in this stage decreases due to increase in the hole concentration.

The understanding kinetic of charge carrier inversion and slow recovery process using diluted air is shown in Figure 83 below: 


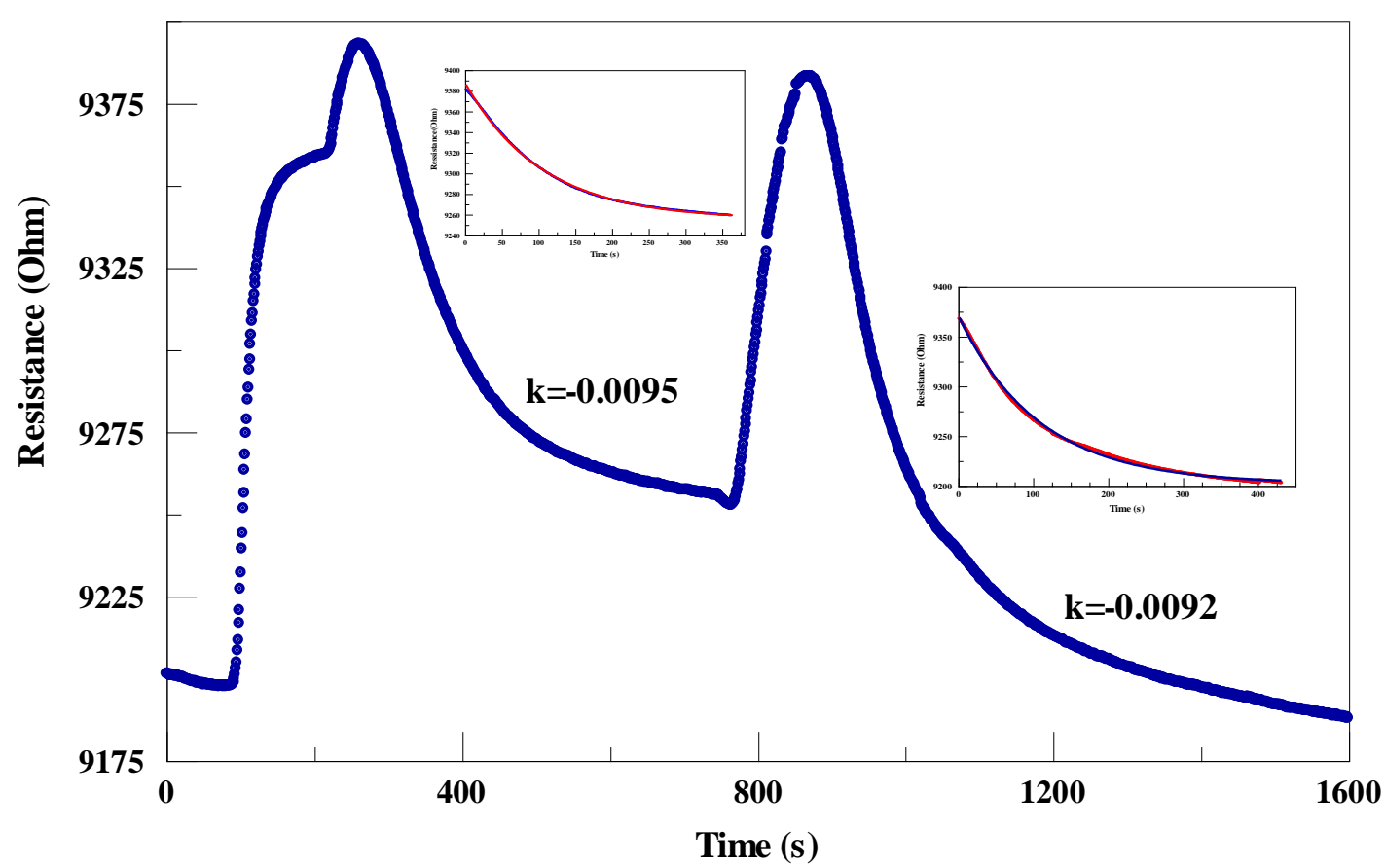

Figure 83: Kinetic of charge carrier inversion and slow recovery using diluted air. The inset shows kinetic fitting curves of stage 3 and 5.

During the charge carrier inversion (stage 3), after reaching maximum depletion thickness corresponding to highest sensor resistance, split atomized hydrogen continues transferring electrons onto nanotube interface. Thus, the interaction and reaction kinetics on the nanotube surface can be represented as:

$$
\begin{aligned}
& H \stackrel{k_{4}}{\rightarrow} H_{\text {surf }}^{+}+e \\
& R=R_{0} e^{-k_{4} t}
\end{aligned}
$$

The rate of electron transfer on the nanotube surface has been reported to be very fast in nanotube based microelectrodes, especially when the electrode has a porous structure and is well wetted by the electrolyte medium. However, it also has been observed that when using individual nanotubes as electrodes, nanotubes do not exhibit good wettability leading to a significant decrease of electron transfer rate [179]. In this 
study, the curve fitting followed equation provides the kinetic constant of electron transfer during this stage $\sim 0.0095 \mathrm{~s}^{-1}$. This is considered to be relatively small. In addition, compared to its counterpart $\left(\mathrm{k}_{2}\right)$ during the exposure process, this value is much smaller. It could be a result of multiple electron transfer stages as well as electronic structure of nanotube layers [180]. In this case, the electrons spilled over and diffusion on nanotube surface is perhaps the rate determining step accounting for a much slower electron transfer rate. Besides, a higher energy barrier attributed to the formation of depletion layer could also be a reason for significant reduction of this rate constant.

Similarly, reaction between protons and $\mathrm{O}_{2}$ molecules by refreshing sensor with air results in resistance decrease in stage 5.

$$
\begin{aligned}
& H_{\text {surf }}^{+}+\mathrm{O}_{2}+e \stackrel{k_{5}}{\rightarrow} \mathrm{HO}_{2} \\
& R=R_{0} e^{k_{5} t}+C
\end{aligned}
$$

The rate constant of this reaction from curve fitting is $\sim 0.0092 \mathrm{~s}^{-1}$. This value is about 10 fold less than its counterpart $\left(\mathrm{k}_{3}\right)$ from previous recovery process using $100 \%$ air. Since this constant change as a function of $\mathrm{O}_{2}$ concentration, they can be represented as:

$$
\begin{aligned}
& k_{3}=K\left(\left[\mathrm{O}_{2}\right]_{100 \%}\right)^{m} \\
& k_{5}=K\left(\left[\mathrm{O}_{2}\right]_{1 \%}\right)^{m}
\end{aligned}
$$

The calculation using values of $k_{3}$ and $k_{5}$ provides $m=\sim 1 / 2$. In other words, the general desorption rate constant of recovery process changes as a function of oxygen concentration and follows: $k_{\text {gen }}=K\left(\left[\mathrm{O}_{2}\right]\right)^{1 / 2}$ 


\subsection{Conclusion}

The current research project provides a simple glass tube method for synthesis of CNTs. The synthetic method involves a clean chemistry whereby no toxic or combustible hydrocarbon gases are used. The method also provides another way to achieve CNTs by using an easy handling solid carbon feed source and offers a promising way to synthesize doped CNTs. In general, naphthalene derivatives which contain the dopant heteroatoms (e.g. B, N etc) atoms were used as targets for the doping. Raman spectroscopy has showed the insertion of impurities into the CNT lattice leads to shifts of D and G bands as well as $\mathrm{D} / \mathrm{G}$ ratio change upon doping.

The sensor, constructed from the incorporation of $\mathrm{Pd}$ nanoparticles with assynthesized CNTs, has shown the ability to detect $\mathrm{H}_{2}$ at ppm levels with a wide dynamic range of detection. The sensing mechanism was based on electron transfer from the interaction between atomized hydrogen and surface of CNTs.

Abnormal sensing behaviors of as-fabricated sensors took place when high $\mathrm{H}_{2}$ concentrations $(>1 \%)$ were used. The characteristic of response curves were associated with charge carrier inversion during the exposure process, by which holes on CNT surface were neutralized by electrons donated from split atomized hydrogen atoms, and then further converted p-type to n-type CNTs. 


\section{CHAPTER 5. FUTURE WORK}

\subsection{Future work of SnO2 NWs}

In this study, the sensor response was based on the change of resistance, and fixed frequency (DC conductance) was used throughout all the measurements. In order to gain deeper understanding about how electrons hop between nanowires, frequency dependent electrical impedance studies should be conducted to probe electron movement in nanoparticles and in nanowires. In addition, there is also a need to look at the possibility to detect other oxidizing gases (such as $\mathrm{NO}_{2}$ and $\mathrm{SO}_{2}$ ) using n-doped $\mathrm{SnO}_{2}$ nanomaterials.

The hybrid structure of $\mathrm{Pd}$ nanoparticles and doped $\mathrm{SnO}_{2}$ nanoparticle/nanowires has been investigated as another material for hydrogen sensors. Preliminary data in Figure 84 shows the detection of this explosive gas at different concentrations at room temperature. The sensors do not display any memory effects observed in sensors, as evidenced by the return of resistance to the baseline after every fresh air purge cycle. The resistance of the sensor quickly decreases upon exposure to hydrogen, followed by a slow increase and slow stabilization and then returns to the initial resistance when exposed to air. Further investigation to understand these interesting response behaviors is needed. 


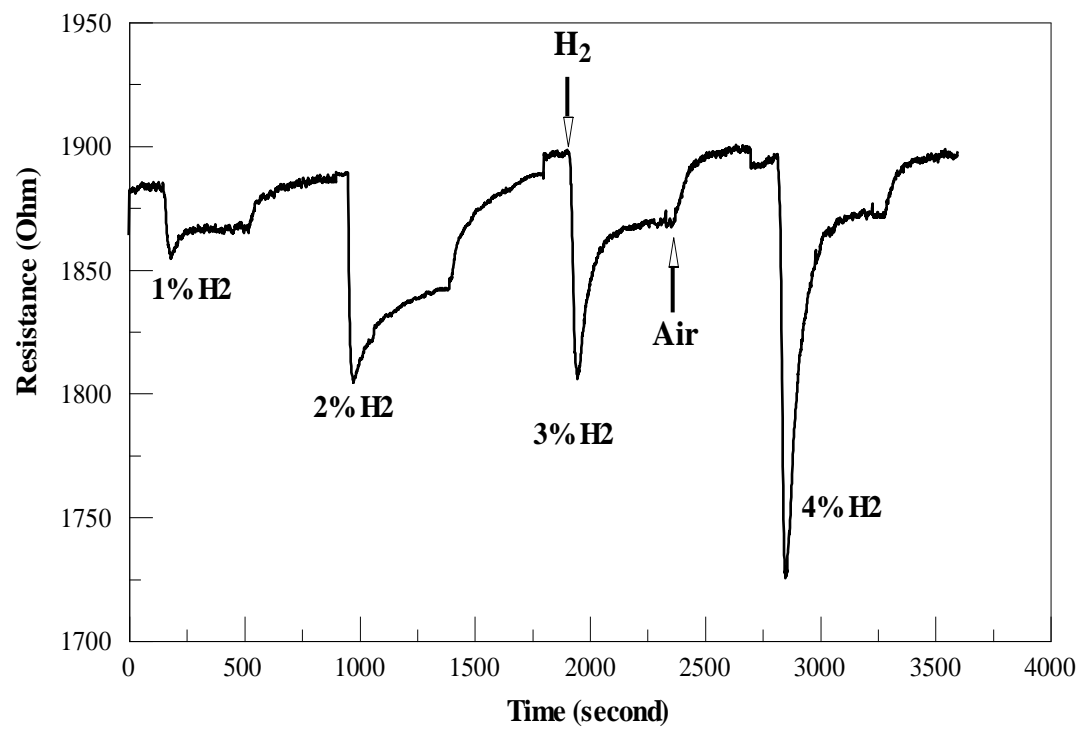

Figure 84: Response of n-doped $\mathrm{SnO}_{2} / \mathrm{Pd}$ (right) sensors to different hydrogen concentrations $\mathrm{H}_{2}$.

\subsection{Future work of CNTs}

Even though Raman spectroscopy shows characteristics of impurities inside a doped CNT lattice, further characterization techniques are still needed to confirm the insertion and determine the percentage of the dopants. Such techniques include electron energy loss spectroscopy (EELS) and X-ray photoelectron spectroscopy. In addition, the dopant concentration in doped CNTs can be detected by Time-of-Flight Secondary Ion Mass Spectroscopy (TOF SIMS). Finally, conductivity measurement using a probe station should be performed to compare the undoped and doped CNTs.

Successful detection of $\mathrm{H}_{2}$ at ppm levels was achieved by the as-constructed sensor. A model to understand the interaction mechanism between exposure gas and the surface of CNTs was established. However, there is still a need to detect and confirm the products of chemical reaction during the recovery process. Besides, in this experiment, the carrier gas was $\mathrm{N}_{2}$, although for a practical sensor $\mathrm{H}_{2}$ diluted in air needs to be 
detected. Such studies should be carried out to investigate the sensor response behaviors with oxygen present at high concentration during the exposure.

Interesting charge carrier inversion was observed from the hybrid structure of Pd NPs and CNTs. The study was carried out based on the sensor system, which is composed from multiple CNTs packed together. Further investigation through studies of individual hybrid structures of Pd NPs-CNT are needed to fully isolate effects of electron flow in single vs collective transport in MWCNTs. Specifically, two as-synthesized Pd NPs decorated MWCNTs can be separated from the bulk material and brought together horizontally on a glass substrate partially sputtered with indium tin oxide (ITO). The local electrical measurement could be performed through atomic force microscopy (AFM) associated with the conducting probe tip, which is brought in contact to one of the tubes. With the setup as showed in Figure 85, a stable DC bias voltage can be applied to the device and measure the current flowing through the tip as $\mathrm{H}_{2}$ with desired concentrations flows into the system. The resulting I-V should be collected and analyzed.

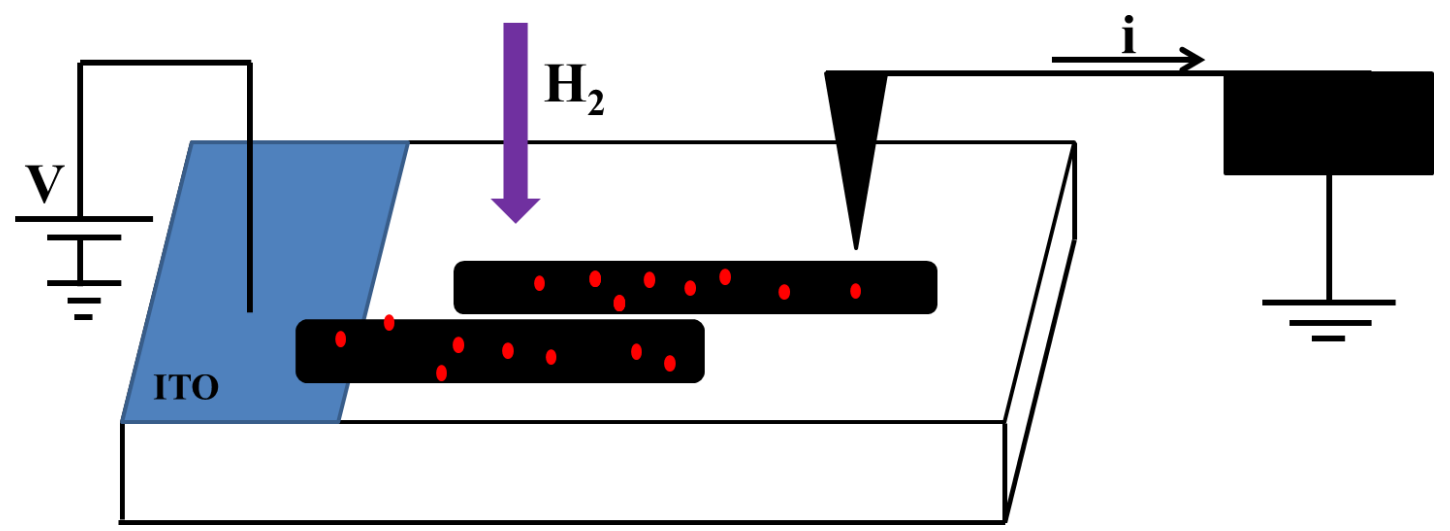

Figure 85: The sketch illustrating experiment for future study of NDR-like behavior using two individuals Pd Np attached CNTs. 
The studies as illustrated from Figure 85 would be a good method to confirm charge carrier inversion observation. It also leads to a better understanding how $\mathrm{p}$ and $\mathrm{n}$ layers are formed within single Pd NPs attached MWCNT during the exposure process, testing the as-proposed mechanism of NDR-like behavior (appendix section D). 


\section{References}

1. Faraday, M., The Bakerian Lecture: Experimental Relations of Gold (and Other Metals) to Light. Phil. Trans. R. Soc. Lond, 1857. 147: p. 145-181.

2. Alagarasi, A., Nanomaterials. VISWANATHAN B ed. 2009.: Narosa Publishing House.

3. Ashby, M.F., P.J. Ferreira, and D.L. Schodek, Chapter 1 - Nanomaterials and Nanotechnologies: An Overview, in Nanomaterials, Nanotechnologies and Design, M.F.A.J.F.L. Schodek, Editor. 2009, Butterworth-Heinemann: Boston. p. 1-16.

4. Sutariya, V.B. and Y. Pathak, Biointeractions of Nanomaterials. 1st ed. 2014, Boca Raton: CRC Press.

5. Harris, P., Carbon Nanotubes and Related Structures. 2001, Cambridge University Press: Cambridge.

6. Sirdeshmukh, D. and L. Sirdeshmukh, Electrical, Electronic and Magnetic Properties of Solids 1st ed. 2014, New York: Springer.

7. Rumyantseva, M.N., et al., Russian Chemical Bulletin, 2003. V52: p. 1217-1238.

8. Zeghbroeck, B.V., Principles of Semiconductor Devices. 2011.

9. Amin, G., $\mathrm{ZnO}$ and $\mathrm{CuO}$ Nanostructures: Low Temperature Growth, Characterization, their Optoelectronic and Sensing Applications in Department of Science and Technology 2012, Linköping University.

10. Huang, X.-J. and Y.-K. Choi, Chemical sensors based on nanostructured materials. Sensors and Actuators B: Chemical, 2007. 122(2): p. 659-671. 
11. Zhou, X.T., et al., Silicon nanowires as chemical sensors. Chemical Physics Letters, 2003. 369(1-2): p. 220-224.

12. Comini, E., et al., Stable and highly sensitive gas sensors based on semiconducting oxide nanobelts. Applied Physics Letters, 2002. 81(10): p. 18691871.

13. Barreca, D., et al., Urchin-like ZnO nanorod arrays for gas sensing applications. CrystEngComm, 2010. 12(11): p. 3419-3421.

14. Kong, J., et al., Nanotube Molecular Wires as Chemical Sensors. Science, 2000. 287(5453): p. 622-625.

15. Cullinan, M.A. and M.L. Culpepper, Carbon nanotubes as piezoresistive microelectromechanical sensors: Theory and experiment. Physical Review B, 2010. 82(11): p. 115428.

16. Jarzebski, Z.M. and J.P. Marton, Physical Properties of SnO Materials. Journal of The Electrochemical Society, 1976. 123: p. C199-C205.

17. Batzill, M. and U. Diebold, The surface and materials science of tin oxide. Progress in Surface Science, 2005. 79(2-4): p. 47-154.

18. Chapadraza, A., Electron Transport and Sensot Characteristics of Tin Dioxide Based Nanocrsytalline Materials in Chemistry. 2009, Portland State University: Portland.

19. Gopel, W., Chemisorption and charge transfer at ionic semiconductor surfaces: Implications in designing gas sensors. Progress in Surface Science, 1985. 20(1): p. 9-103. 
20. Hübert, T., et al., Hydrogen sensors - A review. Sensors and Actuators B: Chemical, 2011. 157(2): p. 329-352.

21. Wang, B., et al., Fabrication of a SnO2 Nanowire Gas Sensor and Sensor Performance for Hydrogen. The Journal of Physical Chemistry C, 2008. 112(17): p. $6643-6647$.

22. Katsuki, A. and K. Fukui, H2 selective gas sensor based on SnO2. Sensors and Actuators B: Chemical, 1998. 52(1-2): p. 30-37.

23. Ramanathan, M., et al., Crossover behavior in the hydrogen sensing mechanism for palladium ultrathin films. Nanotechnology, 2010. 21(12): p. 125501.

24. Fournier, C., et al., Discontinuous Palladium Nanostructures for H2 Sensing. ECS Meeting 2008. 50: p. 3147-3147.

25. Maggie, P., et al., Unprecedented ultra-high hydrogen gas sensitivity in undoped titania nanotubes. Nanotechnology, 2006. 17(2): p. 398.

26. Kolmakov, A., et al., Enhanced Gas Sensing by Individual SnO2 Nanowires and Nanobelts Functionalized with Pd Catalyst Particles. Nano Letters, 2005. 5(4): p. 667-673.

27. Khalap, V.R., et al., Hydrogen Sensing and Sensitivity of Palladium-Decorated Single-Walled Carbon Nanotubes with Defects. Nano Letters, 2010. 10(3): p. 896901.

28. Kong, J., M.G. Chapline, and H. Dai, Functionalized Carbon Nanotubes for Molecular Hydrogen Sensors. Advanced Materials, 2001. 13(18): p. 1384-1386. 
29. Mubeen, S., et al., Palladium Nanoparticles Decorated Single-Walled Carbon Nanotube Hydrogen Sensor. The Journal of Physical Chemistry C, 2007. 111(17): p. 6321-6327.

30. Wang, Y., J.Y. Lee, and T.C. Deivaraj, Controlled Synthesis of V-shaped SnO2 Nanorods. The Journal of Physical Chemistry B, 2004. 108(36): p. 13589-13593.

31. Karousis, N., et al., Carbon Nanotubes Decorated with Palladium Nanoparticles: Synthesis, Characterization, and Catalytic Activity. The Journal of Physical Chemistry C, 2008. 112(35): p. 13463-13469.

32. Abdula, D., Fuctional Surface Chemsitry of Carbon-Based Nanostructures, in Materials Science and Engineering. 2011, University of Illinois at UrbanaChampaign: Urbana.

33. Parker, J.H., D.W. Feldman, and M. Ashkin, Raman Scattering by Silicon and Germanium. Physical Review, 1967. 155(3): p. 712-714.

34. Allen, C. and B.R. Shankar, Room temperature $\mathrm{Cl} 2$ sensing using thick nanoporous films of Sb-doped SnO 2. Nanotechnology, 2008. 19(24): p. 245501.

35. Hamberg, I. and C.G. Granqvist, Evaporated Sn-Doped In2o3 Films - Basic Optical-Properties and Applications to Energy-Efficient Windows. Journal of Applied Physics, 1986. 60(11): p. R123-R159.

36. Hoffman, R.L., B.J. Norris, and J.F. Wager, ZnO-based transparent thin-film transistors. Applied Physics Letters, 2003. 82(5): p. 733-735.

37. Presley, R.E. and et al., Tin oxide transparent thin-film transistors. Journal of Physics D: Applied Physics, 2004. 37(20): p. 2810. 
38. Wager, J.F., Transparent Electronics. Science, 2003. 300(5623): p. 1245-1246.

39. Batley, G.E., A. Ekstrom, and D.A. Johnson, Studies of topochemical heterogeneous catalysis : 3. Catalysis of the reduction of metal oxides by hydrogen. Journal of Catalysis, 1974. 34(3): p. 368-375.

40. Bond, G.C., L.R. Molloy, and M.J. Fuller, Oxidation of carbon monoxide over palladium-tin(IV) oxide catalysts: an example of spillover catalysis. Journal of the Chemical Society, Chemical Communications, 1975(19): p. 796-797.

41. Croft, G. and M.J. Fuller, Water-promoted oxidation of carbon monoxide over tin(IV) oxide-supported palladium. Nature, 1977. 269(5629): p. 585-586.

42. Harrison, P.G., C. Bailey, and W. Azelee, Modified Tin(IV) Oxide $(\mathrm{M} / \mathrm{SnO} 2 \mathrm{M}=\mathrm{Cr}, \mathrm{La}, \mathrm{Pr}, \mathrm{Nd}, \mathrm{Sm}, \mathrm{Gd})$ Catalysts for the Oxidation of Carbon Monoxide and Propane. Journal of Catalysis, 1999. 186(1): p. 147-159.

43. Matsui, T., et al., Effect of reduction-oxidation treatment on the catalytic activity over tin oxide supported platinum catalysts. Science and Technology of Advanced Materials, 2006. 7(6): p. 524-530.

44. Solymosi, F. and J. Kiss, Adsorption and reduction of NO on tin(IV) oxide doped with chromium(III) oxide. Journal of Catalysis, 1978. 54(1): p. 42-51.

45. Allegretti, F., et al., A tin oxide semiconductor sensor for oxygen determination in the sub-ppm range. Sensors and Actuators B: Chemical, 1993. 10(3): p. 191-195.

46. Buttà, N., et al., A family of tin oxide-based sensors with improved selectivity to methane. Sensors and Actuators B: Chemical, 1992. 6(1-3): p. 253-256. 
47. Cané, C., et al., Detection of gases with arrays of micromachined tin oxide gas sensors. Sensors and Actuators B: Chemical, 2000. 65(1-3): p. 244-246.

48. Chaparadza, A. and S.B. Rananavare, Room temperature Cl 2 sensing using thick nanoporous films of Sb-doped SnO 2. Nanotechnology, 2008. 19(24): p. 245501.

49. Tamaki, J., et al., $\mathrm{CuO}-\mathrm{SnO} 2$ element for highly sensitive and selective detection of H2S. Sensors and Actuators B: Chemical, 1992. 9(3): p. 197-203.

50. Wu, J.M., A room temperature ethanol sensor made from p-type Sb-doped $\mathrm{SnO} 2$ nanowires. Nanotechnology, 2010. 21(23): p. 235501.

51. Baur, W.H., 1956. 9: p. 515-529.

52. Jarzebski, Z.M., Oxide Semiconductors,. 1973, Oxford-Newyork: Pergamon Press.

53. Amma, D.S.D., V.K. Vaidyan, and P.K. Manoj, Structural, electrical and optical studies on chemically deposited tin oxide films from inorganic precursors. Materials Chemistry and Physics, 2005. 93(1): p. 194-201.

54. Kröger, F.A. and H.J. Vink, Relations between the Concentrations of Imperfections in Crystalline Solids, in Solid State Physics, S. Frederick and T. David, Editors. 1956, Academic Press. p. 307-435.

55. Braithwaite, N. and G. Weaver, Electronic Materials. 1990, Oxford: ButterworthHeinemann.

56. Dieguez, A., et al., The complete Raman spectrum of nanometric SnO[sub 2] particles. Journal of Applied Physics, 2001. 90(3): p. 1550-1557. 
57. Merle, P., et al., Uniaxial-stress dependence of the first-order Raman spectrum of rutile. I. Experiments. Physical Review B, 1980. 21(4): p. 1617-1626.

58. Sangeetha, P., V. Sasirekha, and V. Ramakrishnan, Micro-Raman investigation of tin dioxide nanostructured material based on annealing effect. Journal of Raman Spectroscopy, 2011. 42(8): p. 1634-1639.

59. Hu, J.Q., et al., Laser-Ablation Growth and Optical Properties of Wide and Long Single-Crystal SnO2 Ribbons. Advanced Functional Materials, 2003. 13(6): p. 493-496.

60. Liu, C.M., et al., Fabrication and characterization of wire-like $S n O$ 2. Journal of Physics D: Applied Physics, 2006. 39(12): p. 2494.

61. Kim, H., J. Lee, and C. Lee, Growth of tin oxide rod-like and sheet-like structures. Journal of Materials Science: Materials in Electronics, 2009. 20(2): p. 99-104.

62. Pan, Z.W., Z.R. Dai, and Z.L. Wang, Nanobelts of Semiconducting Oxides. Science, 2001. 291(5510): p. 1947-1949.

63. Mathur, S. and S. Barth, Molecule-Based Chemical Vapor Growth of Aligned SnO2 Nanowires and Branched SnO2/V2O5 Heterostructures. Small, 2007. 3(12): p. 2070-2075.

64. Chen, Y., et al., Bulk-quantity synthesis and self-catalytic VLS growth of SnO2 nanowires by lower-temperature evaporation. Chemical Physics Letters, 2003. 369(1-2): p. 16-20. 
65. Nguyen, P., et al., Epitaxial Directional Growth of Indium-Doped Tin Oxide Nanowire Arrays. Nano Letters, 2003. 3(7): p. 925-928.

66. Wang, J.X., et al., Growth of SnO2 nanowires with uniform branched structures. Solid State Communications, 2004. 130(1-2): p. 89-94.

67. Ma, X.L., Y. Li, and Y.L. Zhu, Growth mode of the SnO2 nanobelts synthesized by rapid oxidation. Chemical Physics Letters, 2003. 376(5-6): p. 794-798.

68. Wang, W., J. Niu, and L. Ao, Large-scale synthesis of single-crystal rutile SnO2 nanowires by oxidizing SnO nanoparticles in flux. Journal of Crystal Growth, 2008. 310(2): p. 351-355.

69. Wang, Y. and J.Y. Lee, Molten Salt Synthesis of Tin Oxide Nanorods:â€\%o Morphological and Electrochemical Features. The Journal of Physical Chemistry B, 2004. 108(46): p. 17832-17837.

70. $\mathrm{Yu}, \mathrm{C}$. , et al., Growth of single-crystalline SnO2 nanocubes via a hydrothermal route. CrystEngComm, 2010. 12(2): p. 341-343.

71. Yu, S.-H., et al., A Novel Solventothermal Synthetic Route to Nanocrystalline CdE $(E=S, S e, T e)$ and Morphological Control. Chemistry of Materials, 1998. 10(9): p. $2309-2312$.

72. Adamson, A.W., Physical Chemistry of Surfaces. 1997. 808.

73. Guifu, Z., et al., Solvothermal/hydrothermal route to semiconductor nanowires. Nanotechnology, 2006. 17(11): p. S313.

74. Herring, C., Some Theorems on the Free Energies of Crystal Surfaces. Physical Review, 1951. 82(1): p. 87-93. 
75. Venables, J.A., Introduction to Surface and Thin Film Processes. 2000, Cambridge: Cambridge University Press. 4.

76. Morin, S.A., et al., Mechanism and Kinetics of Spontaneous Nanotube Growth Driven by Screw Dislocations. Science, 2010. 328(5977): p. 476-480.

77. Zemel, J.N., Theoretical description of gas-film interaction on SnOx. Thin Solid Films, 1988. 163: p. 189-202.

78. Sánchez-González, J., et al., Electrical conductivity of carbon blacks under compression. Carbon, 2005. 43(4): p. 741-747.

79. Chaparadza, A., S.B. Rananavare, and V. Shutthanandan, Synthesis and characterization of lithium-doped tin dioxide nanocrystalline powders. Materials Chemistry and Physics, 2007. 102(2-3): p. 176-180.

80. Jung-Sub, L., S. Vaidyanathan, and D.Y. Jeon. Formation of Sn-multiwalled carbon nanotube composite layer for the application of thermal interface materials. in Electronic Materials and Packaging, 2007. EMAP 2007. International Conference on. 2007.

81. Majumdar, S., et al., Room temperature synthesis of nanocrystalline SnO through sonochemical route. Materials Letters, 2008. 62(8-9): p. 1249-1251.

82. Chan, J.C., et al., Facile pyrolytic synthesis of silicon nanowires. Solid-State Electronics, 2010. 54(10): p. 1185-1191.

83. Kim, T.W., D.U. Lee, and Y.S. Yoon, Microstructural, electrical, and optical properties of SnO[sub 2] nanocrystalline thin films grown on InP (100) substrates 
for applications as gas sensor devices. Journal of Applied Physics, 2000. 88(6): p. $3759-3761$.

84. Wang, B., et al., Field emission and photoluminescence of SnO[sub 2] nanograss. Journal of Applied Physics, 2005. 98(12): p. 124303-4.

85. Tran, H.A. and S.B. Rananavare, Synthesis and characterization of $N$ - and Pdoped tin oxide nanowires, in 2011 11th IEEE International Conference on Nanotechnology (2011). 2011, IEEE: Portland, OR. p. 144-149.

86. Chopra, K.L., S. Major, and D.K. Pandya, Transparent conductors-A status review. Thin Solid Films, 1983. 102(1): p. 1-46.

87. Wang, Y., I. Ramos, and J.J. Santiago-Aviles, Optical bandgap and photoconductance of electrospun tin oxide nanofibers. Journal of Applied Physics, 2007. 102(9): p. 093517-5.

88. Ji, Z., et al., A novel transparent pn+ junction based on indium tin oxides. Thin Solid Films, 2004. 460(1-2): p. 324-326.

89. Ahmed, A.S., et al., Band gap narrowing and fluorescence properties of nickel doped SnO2 nanoparticles. Journal of Luminescence, 2011. 131(1): p. 1-6.

90. Johnson, P.L. and D. Teeters, Formation and characterization of SnO2 nanobaskets. Solid State Ionics, 2006. 177(26-32): p. 2821-2825.

91. Abello, L., et al., Structural Characterization of Nanocrystalline SnO2by X-Ray and Raman Spectroscopy. Journal of Solid State Chemistry, 1998. 135(1): p. 7885. 
92. Gouadec, G.e. and P. Colomban, Raman Spectroscopy of Nanomaterials: How Spectra Relate to Disorder, Particle Size and Mechanical Properties. Crystal Growth and Characterization of Materials, 2007. 53: p. 1-56.

93. Luo, Z.-H., et al., Controlling the electronic structure of SnO 2 nanowires by Modoping. Chinese Physics B, 2010. 19(2): p. 026102.

94. Chapadraza, A., Eectron Transport and Sensor Characteristics of Tin Oxide Based Nanocrystalline Material, in Chemistry. 2009, Poatland State University: Portland.

95. Jain, G.H., L.A. Patil, and V.B. Gaikwad, Studies on gas sensing performance of (Ba0.8Sr0.2)(Sn0.8Ti0.2)O3 thick film resistors. Sensors and Actuators B: Chemical, 2007. 122(2): p. 605-612.

96. Somorjai, G.A. and Y. Li, Introduction to Surface Chemistry and Catalysis. 2010: John Wiley and Sons.

97. Bender, F., et al., Characterization of a WO3 thin film chlorine sensor. Sensors and Actuators B: Chemical, 2001. 77(1-2): p. 281-286.

98. Zhao, W., et al., Sensitive properties of In-based compound semiconductor oxide to Cl 2 gas. Journal of Semiconductors, 2009. 30(3): p. 034010.

99. Joshi, A., et al., ZnO-nanowires modified polypyrrole films as highly selective and sensitive chlorine sensors. Applied Physics Letters, 2009. 94(10): p. -.

100. Patil, D.R. and L.A. Patil, Room temperature chlorine gas sensing using surface modified ZnO thick film resistors. Sensors and Actuators B: Chemical, 2007. 123(1): p. 546-553. 
101. Dawson, D.H. and D.E. Williams, Gas-sensitive resistors: surface interaction of chlorine with semiconducting oxides. Journal of Materials Chemistry, 1996. 6(3): p. $409-414$.

102. Chan, J.C., et al., Mechanisms of Aging of Antimony Doped Tin Oxide Based Electrochromic Devices. Japanese Journal of Applied Physics, 2006 45(49): p. L1300-L1303.

103. Szczuko, D., et al., XPS investigations of surface segregation of doping elements in SnO2. Applied Surface Science, 2001. 179(1-4): p. 301-306.

104. Egdell, R.G., W.R. Flavell, and P. Tavener, Antimony-doped tin(IV) oxide: Surface composition and electronic structure. Journal of Solid State Chemistry, 1984. 51(3): p. 345-354.

105. McGinley, C., et al., Pure and Sb-doped SnO 2 nanoparticles studied by photoelectron spectroscopy. The European Physical Journal D - Atomic, Molecular, Optical and Plasma Physics, 2001. 16(1): p. 225-228.

106. Dusastre, V. and D.E. Williams, Sb(III) as a Surface Site for Water Adsorption on $\mathrm{Sn}(\mathrm{Sb}) \mathrm{O2}$, and Its Effect on Catalytic Activity and Sensor Behavior. The Journal of Physical Chemistry B, 1998. 102(35): p. 6732-6737.

107. Kamble, R.B. and V.L. Mathe, Nanocrystalline nickel ferrite thick film as an efficient gas sensor at room temperature. Sensors and Actuators B: Chemical, 2008. 131(1): p. 205-209. 
108. Zhao, X., et al., Room-temperature chlorine gas sensor based on CdSnO3 synthesized by hydrothermal process. Journal of Advanced Ceramics, 2013. 2(1): p. 31-36.

109. Inderwildi, O.R. and M. Kraft, Adsorption, Diffusion and Desorption of Chlorine on and from Rutile TiO2\{110\}: A Theoretical Investigation. ChemPhysChem, 2007. 8(3): p. 444-451.

110. Rochkind, M.M. and G.C. Pimentel, Infrared Spectrum and Vibrational Assignment for Chlorine Monoxide, Cl2O. The Journal of Chemical Physics, 1965. 42(4): p. 1361-1368.

111. Schack, C.J. and D. Pilipovich, Chlorine perchlorate. Inorganic Chemistry, 1970. 9(6): p. 1387-1390.

112. Christe, K.O., C.J. Schack, and E.C. Curtis, Halogen perchlorates. Vibrational spectra. Inorganic Chemistry, 1971. 10(8): p. 1589-1593.

113. Mueller, H.S.P. and H. Willner, Vibrational and electronic spectra of chlorine dioxide, OClO, and chlorine superoxide $\mathrm{ClOO}$, isolated in cryogenic matrixes. The Journal of Physical Chemistry, 1993. 97(41): p. 10589-10598.

114. Avouris, P., Carbon nanotube electronics. Chemical Physics, 2002. 281(2-3): p. 429-445.

115. Dag, S., et al., - Electronic structure of the contact between carbon nanotube and metal electrodes. 2003.

116. Journet, C., et al., Large-scale production of single-walled carbon nanotubes by the electric-arc technique. Nature, 1997. 388(6644): p. 756-758. 
117. Fonseca, A. and J.B. Nagy, Carbon Nanotubes Formation in the Arc Discharge Process, in Carbon Filaments and Nanotubes: Common Origins, Differing Applications?, L.P. Biró, et al., Editors. 2001, Springer Netherlands. p. 75-84.

118. Suzuki, S., Syntheses and Applications of Carbon Nanotubes and Their Composites. 2013: InTech.

119. Guo, T., et al., Self-Assembly of Tubular Fullerenes. The Journal of Physical Chemistry, 1995. 99(27): p. 10694-10697.

120. Sinnott, S.B. and R. Andrews, Carbon Nanotubes: Synthesis, Properties, and Applications. Critical Reviews in Solid State and Material Sciences, 2001. 26(3): p. $145-249$.

121. Seo, J.W. and et al., Catalytically grown carbon nanotubes: from synthesis to toxicity. Journal of Physics D: Applied Physics, 2007. 40(6): p. R109.

122. Paradise, M. and T. Goswami, Carbon nanotubes - Production and industrial applications. Materials \& Design, 2007. 28(5): p. 1477-1489.

123. Mathur, R.B., S. Chatterjee, and B.P. Singh, Growth of carbon nanotubes on carbon fibre substrates to produce hybrid/phenolic composites with improved mechanical properties. Composites Science and Technology, 2008. 68: p. 16081615.

124. Schmidt, V., et al., Silicon Nanowires: A Review on Aspects of their Growth and their Electrical Properties. Advanced Materials, 2009. 21(25-26): p. 2681-2702. 
125. Doorn, S.K., et al., Resonant Raman excitation profiles of individually dispersed single walled carbon nanotubes in solution. Applied Physics A, 2004. 78(8): p. 1147-1155.

126. Rao, A.M., et al., Diameter-Selective Raman Scattering from Vibrational Modes in Carbon Nanotubes. Science, 1997. 275(5297): p. 187-191.

127. Tuinstra, F. and J.L. Koenig, Raman Spectrum of Graphite. The Journal of Chemical Physics, 1970. 53(3): p. 1126-1130.

128. Dresselhaus, M.S., et al., Raman spectroscopy on isolated single wall carbon nanotubes. Carbon, 2002. 40(12): p. 2043-2061.

129. Jang, Y.-T., et al., A simple approach in fabricating chemical sensor using laterally grown multi-walled carbon nanotubes. Sensors and Actuators B: Chemical, 2004. 99(1): p. 118-122.

130. Dresselhaus, M.S., et al., Raman spectroscopy of carbon nanotubes. Physics Reports, 2005. 409(2): p. 47-99.

131. Ewels, C. (2007) Doping of Carbon Nanotubes. Volume, DOI: http://www.ewels.info/science/publications/papers/2008.DopingChapter.pdf

132. Zhou, O., et al., Defects in Carbon Nanostructures. Science, 1994. 263(5154): p. 1744-1747.

133. Mordkovich, V.Z., et al., Intercalation into carbon nanotubes. Carbon, 1996. 34(10): p. 1301-1303.

134. Nalimova, V.A., et al., Lithium interaction with carbon nanotubes. Synthetic Metals, 1997. 88(2): p. 89-93. 
135. Maurin, G., et al., Electrochemical intercalation of lithium into multiwall carbon nanotubes. Chemical Physics Letters, 1999. 312(1): p. 14-18.

136. Hamwi, A., et al., Fluorination of carbon nanotubes. Carbon, 1997. 35(6): p. 723728.

137. Jin, Z.-X., G.Q. Xu, and S.H. Goh, A preferentially ordered accumulation of bromine on multi-wall carbon nanotubes. Carbon, 2000. 38(8): p. 1135-1139.

138. Stephan, O., et al., Doping Graphitic and Carbon Nanotube Structures with Boron and Nitrogen. Science, 1994. 266(5191): p. 1683-1685.

139. Kim, S.Y., et al., X-ray Photoelectron Spectroscopy and First Principles Calculation of BCN Nanotubes. Journal of the American Chemical Society, 2007. 129(6): p. 1705-1716.

140. Hsu, W.K., et al., Metallic behaviour of boron-containing carbon nanotubes. Chemical Physics Letters, 2000. 323(5-6): p. 572-579.

141. Terrones, M., et al., N-doping and coalescence of carbon nanotubes: synthesis and electronic properties. Applied Physics A: Materials Science \&amp; Processing, 2002. 74(3): p. 355-361.

142. Kudashov, A.G., et al., Influence of Ni-Co Catalyst Composition on Nitrogen Content in Carbon Nanotubes. The Journal of Physical Chemistry B, 2004. 108(26): p. 9048-9053.

143. Maldonado, S., S. Morin, and K.J. Stevenson, Structure, composition, and chemical reactivity of carbon nanotubes by selective nitrogen doping. Carbon, 2006. 44(8): p. 1429-1437. 
144. Liu, J., S. Webster, and D.L. Carroll, Highly aligned coiled nitrogen-doped carbon nanotubes synthesized by injection-assisted chemical vapor deposition. Applied Physics Letters, 2006. 88(21): p. - .

145. Ruiz-Soria, G., et al., On the purification of CVD grown boron doped singlewalled carbon nanotubes. physica status solidi (b), 2011. 248(11): p. 2504-2507.

146. Modi, A., et al., Miniaturized gas ionization sensors using carbon nanotubes. Nature, 2003. 424(6945): p. 171-174.

147. Penza, M., F. Antolini, and M.V. Antisari, Carbon nanotubes as SAW chemical sensors materials. Sensors and Actuators B: Chemical, 2004. 100(1-2): p. 47-59.

148. Peng, N., et al., Sensing Mechanisms for Carbon Nanotube Based NH3 Gas Detection. Nano Letters, 2009. 9(4): p. 1626-1630.

149. Shu, P. and C. Kyeongjae, Chemical control of nanotube electronics. Nanotechnology, 2000. 11(2): p. 57.

150. Feng, X., et al., Sensitivity of Ammonia Interaction with Single-Walled Carbon Nanotube Bundles to the Presence of Defect Sites and Functionalities. Journal of the American Chemical Society, 2005. 127(30): p. 10533-10538.

151. Robinson, J.A., et al., Role of Defects in Single-Walled Carbon Nanotube Chemical Sensors. Nano Letters, 2006. 6(8): p. 1747-1751.

152. Avouris, P., M. Freitag, and V. Perebeinos, Carbon-nanotube photonics and optoelectronics. Nat Photon, 2008. 2(6): p. 341-350.

153. Andzelm, J., N. Govind, and A. Maiti, Nanotube-based gas sensors - Role of structural defects. Chemical Physics Letters, 2006. 421(1-3): p. 58-62. 
154. Chan, J.C., A novel approach to the synthesis of silicon nanowires. 2008, Portland State University: Portland.

155. Kumar, M. and Y. Ando, Chemical vapor deposition of carbon nanotubes: $a$ review on growth mechanism and mass production. J Nanosci Nanotechnol., 2010. 10(6): p. 3739-58.

156. Nagaraju, N., et al., Alumina and silica supported metal catalysts for the production of carbon nanotubes. Journal of Molecular Catalysis A: Chemical, 2002. 181(1-2): p. 57-62.

157. Ago, H., et al., Roles of Metal-Support Interaction in Growth of Single- and Double-Walled Carbon Nanotubes Studied with Diameter-Controlled Iron Particles Supported on $\mathrm{MgO}$. The Journal of Physical Chemistry B, 2004. 108(49): p. 18908-18915.

158. Dresselhaus, M.S., et al., Perspectives on Carbon Nanotubes and Graphene Raman Spectroscopy. Nano Letters, 2010. 10(3): p. 751-758.

159. Ceragioli, H.J., et al., Synthesis and characterization of boron-doped carbon nanotubes. Journal of Physics: Conference Series, 2008. 100(5): p. 052029.

160. Maciel, I.O., et al., Boron, nitrogen and phosphorous substitutionally doped single-wall carbon nanotubes studied by resonance Raman spectroscopy. physica status solidi (b), 2009. 246(11-12): p. 2432-2435.

161. Sharifi, T., et al., Nitrogen doped multi walled carbon nanotubes produced by CVD-correlating XPS and Raman spectroscopy for the study of nitrogen inclusion. Carbon, 2012. 50(10): p. 3535-3541. 
162. Yadav, R., et al., Effect of Growth Temperature on Bamboo-shaped CarbonNitrogen (C-N) Nanotubes Synthesized Using Ferrocene Acetonitrile Precursor. Nanoscale Research Letters, 2008. 4(3): p. 197 - 203.

163. McGuire, K., et al., Synthesis and Raman characterization of boron-doped singlewalled carbon nanotubes. Carbon, 2005. 43(2): p. 219-227.

164. Shim, M., et al., Spectral Diversity in Raman G-band Modes of Metallic Carbon Nanotubes within a Single Chirality. The Journal of Physical Chemistry C, 2008. 112(33): p. 13017-13023.

165. Bulusheva, L.G., et al., Effect of nitrogen doping on Raman spectra of multiwalled carbon nanotubes. physica status solidi (b), 2008. 245(10): p. 1971-1974.

166. Fagan, S.B., et al., Electronic properties of Ag- and CrO3-filled single-wall carbon nanotubes. Chemical Physics Letters, 2005. 406(1-3): p. 54-59.

167. Pisana, S., et al., Breakdown of the adiabatic Born-Oppenheimer approximation in graphene. Nat Mater, 2007. 6(3).

168. Yan, J., et al., Electric Field Effect Tuning of Electron-Phonon Coupling in Graphene. Physical Review Letters, 2007. 98(16): p. 166802.

169. Das, A., B. Chakraborty, and A.K. Sood, Raman spectroscopy of graphene on different substrates and influence of defects. Bull. Mater. Sci, 2007. 31(3).

170. Tierney, H.L., et al., Hydrogen Dissociation and Spillover on Individual Isolated Palladium Atoms. Physical Review Letters, 2009. 103(24): p. 246102. 
171. Parambhath, V.B., et al., Investigation of Spillover Mechanism in Palladium Decorated Hydrogen Exfoliated Functionalized Graphene. The Journal of Physical Chemistry C, 2011. 115(31): p. 15679-15685.

172. Donghun, K., et al., Oxygen-induced p-type doping of a long individual singlewalled carbon nanotube. Nanotechnology, 2005. 16(8): p. 1048.

173. Derycke, V., et al., Controlling doping and carrier injection in carbon nanotube transistors. Applied Physics Letters, 2002. 80(15): p. 2773-2775.

174. Collins, P.G., et al., Extreme Oxygen Sensitivity of Electronic Properties of Carbon Nanotubes. Science, 2000. 287(5459): p. 1801-1804.

175. Mandelis, A. and C. Christofides, Physics, Chemistry and Technology of Solid State Gas Sensor Devices. 1993, New York: Wiley.

176. Kaniyoor, A., T.T. Baby, and S. Ramaprabhu, Graphene synthesis via hydrogen induced low temperature exfoliation of graphite oxide. Journal of Materials Chemistry, 2010. 20(39): p. 8467-8469.

177. Nikitin, A., et al., Hydrogen Storage in Carbon Nanotubes through the Formation of Stable C-H Bonds. Nano Letters, 2007. 8(1): p. 162-167.

178. Gao, W., et al., New insights into the structure and reduction of graphite oxide. Nat Chem, 2009. 1(5): p. 403-408.

179. Nugent, J.M., et al., Fast Electron Transfer Kinetics on Multiwalled Carbon Nanotube Microbundle Electrodes. Nano Letters, 2001. 1(2): p. 87-91.

180. Ajayan, P.M., Nanotubes from Carbon. Chemical Reviews, 1999. 99(7): p. 17871800. 


\section{Appendix A. Chemical reagents}

Tin chloride pentahydrate

Sodium borohydride $\left(\mathrm{NaBH}_{4}\right)$

Sodium chloride

Potassium chloride

Lithium chloride

1,10 Phenanthroline monohydrate

Naphthalene

Ferrocene

Cobaltocene

1277-43-6, $98 \%$

Dimethylformamide (DMF)

Antimony trichloride $\left(\mathrm{SbCl}_{3}\right)$

$99.5 \%$

Triphenylborane (TPB)

Benzylamine

Sodium dodecyl sulfate $98 \%$

Palladium acetate 3375-31-3, powder, $98 \%$
Sigma-Aldrich, CAS No. 10026-06-9, >99.8\%

Sigma-Aldrich, CAS No. 16940-66-2, powder, $>98.5 \%$

Sigma-Aldrich, CAS No. 7647-14-5, powder, >99\%

Sigma-Aldrich, CAS No. 7447-40-7, powder, >99\%

Sigma-Aldrich, CAS No. 7447-41-8, powder, >99\%

Sigma-Aldrich, CAS No. 5144-89-8, powder, 99\%

Sigma-Aldrich, CAS No. 91-20-3, crystal, 99\%

Sigma-Aldrich, CAS No. 102-54-5, powder, 99\%

Acros Organic, CAS No.

Sigma-Aldrich, CAS No. 68-12-2, >99.8\%

Acros Organic, CAS No. 10025-91-9,

Sigma-Aldrich, CAS no. 960-71-4, $<2 \% \mathrm{H}_{2} \mathrm{O}$

Sigma-Aldrich, CAS no. 100-46-9, 99\%

Sigma-Aldrich, CAS No. 151-21-3, powder, Sigma-Aldrich, CAS No. 


\section{Appendix B. Characterization of $\mathrm{SnO}_{2} \mathrm{NWs}$ at different synthetic conditions}

The morphology of $\mathrm{SnO}_{2} \mathrm{NWs}$ was investigated at different synthetic conditions including temperature, environment and the presence of surfactant, 1,10 phenanthroline. In this study, the syntheses were carried out using method $\mathrm{C}$ (section 2.7.1.1). SEM was employed for the characterization, examining diameter and length of $\mathrm{SnO}_{2} \mathrm{NW}$ formation.

Size histograms of diameter and length of nanowires were analyzed based on standard Gaussian distribution function. The results are showed in the following table B.1

$$
G(x)=a \cdot e^{-b(x-c)^{2}}+d
$$

Where

$c$ is the fitting parameter (length or diameter)

$a, b, d$ are fitting constants

Table B.1: The effect of synthetic conditions on $\mathrm{SnO} 2 \mathrm{NW}$ s morphology (O and NO stand for observed and non-observed, respectively)

\begin{tabular}{|c|c|c|c|c|}
\hline Synthetic condition of $\mathrm{SnO} 2 \mathrm{NWs}$ & $\begin{array}{c}\text { Length } \\
(\mathrm{nm})\end{array}$ & $\begin{array}{c}\text { Gaussian } \\
\text { Distribution } \\
\left(\mathrm{b} \times 10^{6}\right)\end{array}$ & $\begin{array}{c}\text { Diameter } \\
(\mathrm{nm})\end{array}$ & $\begin{array}{c}\text { Gaussian } \\
\text { Distribution } \\
\left(\mathrm{b} \times 10^{6}\right.\end{array}$ \\
\hline $\mathrm{SnO}_{\mathrm{x}} \mathrm{nps}+\mathrm{N}_{2}+490 \mathrm{C}$ & $\mathrm{NO}$ & $\mathrm{NO}$ & $\mathrm{NO}$ & $\mathrm{NO}$ \\
\hline $\mathrm{SnO}_{\mathrm{x}} \mathrm{nps}+\mathrm{N}_{2}+\mathrm{Phen}+490^{\circ} \mathrm{C}$ & $\mathrm{O}$ & 0 & $20 \pm 2$ & $47 \pm 1$ \\
\hline $\mathrm{Dilute} \mathrm{SnO}+490^{\circ} \mathrm{C}$ & 925 & $80 \pm 5$ & $125 \pm 6$ & $300 \pm 50$ \\
\hline $\mathrm{Snnps}+\mathrm{N}_{2}+490^{\circ} \mathrm{C}$ & $240 \pm 3$ & $81 \pm 9$ & $45.8 \pm 5$ & $210 \pm 20$ \\
\hline $\mathrm{Snnps}+\mathrm{Air}+490^{\circ} \mathrm{C}$ & $292 \pm 3$ & $400 \pm 100$ & $21.3 \pm 3$ & $2100 \pm 300$ \\
\hline $\mathrm{Sn}+\mathrm{Air}+\mathrm{Phen}+490^{\circ} \mathrm{C}$ & $407 \pm 4$ & $81 \pm 9$ & $129 \pm 3$ & $210 \pm 20$ \\
\hline $\mathrm{Snnps}+\mathrm{Air}+700^{\circ} \mathrm{C}$ & $800 \pm 20$ & $12 \pm 4$ & $72 \pm 2$ & $120 \pm 20$ \\
\hline
\end{tabular}

In the Gibbs nucleation model, the term $b$ is related to the surface energy of nucleating cluster. Therefore, the surface free energies of the clusters corresponding to two dimensions (length and diameter) can be estimated. 


$$
b \sim \frac{4 \pi \gamma}{3 k_{B} T}
$$

Table B.2: Calculation of the surface free energies of cluster nucleation

\begin{tabular}{|c|c|c|c|c|}
\hline $\begin{array}{c}\text { Synthetic condition of SnO2 } \\
\text { NWs }\end{array}$ & $\begin{array}{c}\text { Gaussian } \\
\text { Distribution of } \\
\text { length }\left(\mathrm{b} \times 10^{6}\right)\end{array}$ & $\begin{array}{c}\gamma_{\mathrm{L}}\left(\mathrm{Nm}^{-1}\right) \\
\left(\gamma \times 10^{26}\right)\end{array}$ & $\begin{array}{c}\text { Gaussian } \\
\text { Distribution of } \\
\text { diameter }\left(\mathrm{b} \times 10^{6}\right)\end{array}$ & $\begin{array}{c}\gamma_{\mathrm{D}}\left(\mathrm{Nm}^{-1}\right) \\
\left(\gamma \times 10^{26}\right)\end{array}$ \\
\hline $\mathrm{SnO}_{\mathrm{x}} \mathrm{nps}+\mathrm{N}_{2}+\mathrm{Phen}+490^{\circ} \mathrm{C}$ & 0 & 0 & $47 \pm 1$ & $11 \pm 1$ \\
\hline Dilute $\mathrm{SnO}+490^{\circ} \mathrm{C}$ & $80 \pm 5$ & $20 \pm 1$ & $300 \pm 50$ & $75 \pm 1$ \\
\hline $\mathrm{Snnps}+\mathrm{N}_{2}+490^{\circ} \mathrm{C}$ & $81 \pm 9$ & $20 \pm 2$ & $210 \pm 20$ & $52 \pm 5$ \\
\hline $\mathrm{Snnps}+\mathrm{Air}+490^{\circ} \mathrm{C}$ & $400 \pm 100$ & $100 \pm 3$ & $2100 \pm 300$ & $528 \pm 75$ \\
\hline $\mathrm{Sn}+$ Air+ Phen $+490^{\circ} \mathrm{C}$ & $81 \pm 9$ & $20 \pm 2$ & $210 \pm 20$ & $210 \pm 5$ \\
\hline $\mathrm{Sn} \mathrm{nps}+\mathrm{Air}+700^{\circ} \mathrm{C}$ & $12 \pm 4$ & $4 \pm 1$ & $120 \pm 20$ & $120 \pm 5$ \\
\hline
\end{tabular}
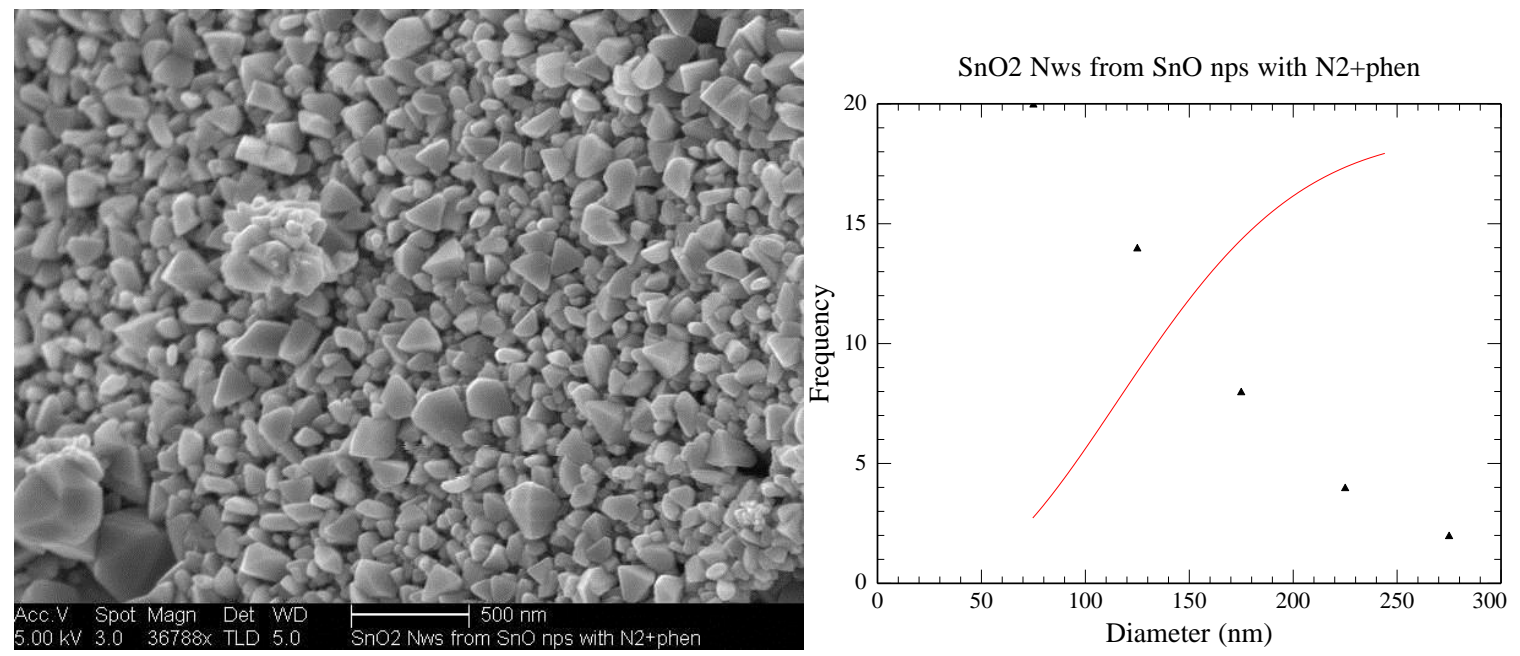

Figure B1: $\mathrm{SnO}_{2} \mathrm{NWs}$ synthesized from $\mathrm{SnO}_{\mathrm{x}} \mathrm{NPs}$ at $490^{\circ} \mathrm{C}$ in an $\mathrm{N}_{2}$ environment with the presence of 1,10 phenanthroline. Nanowires with small length were observed from this synthetic condition, but not fitted by Gaussian distribution. 


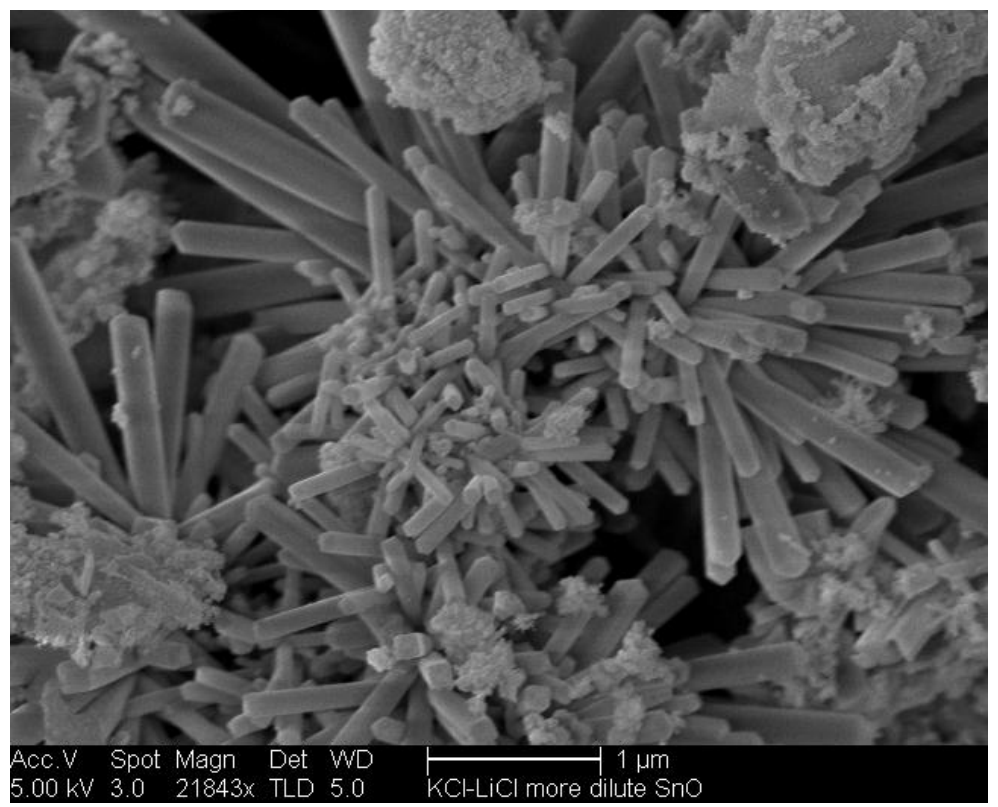

$\mathrm{KCl}-\mathrm{LiCl}$ more dilute $\mathrm{SnO}$

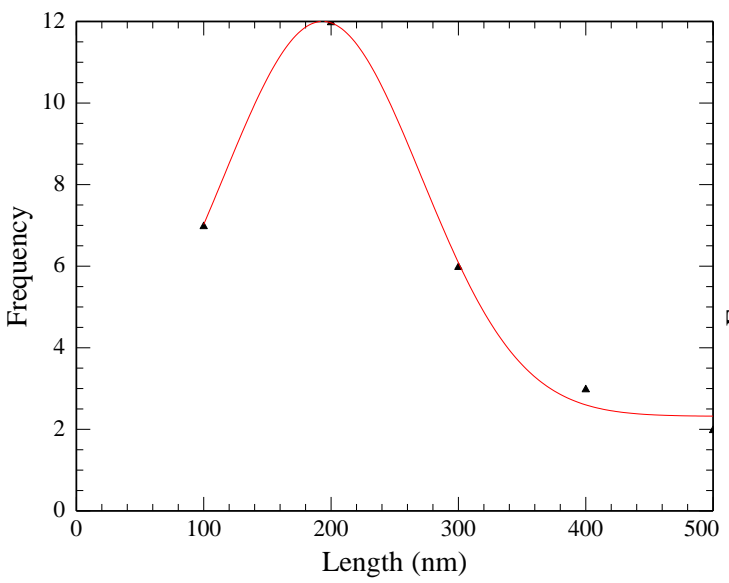

$\mathrm{KCl}-\mathrm{LiCl}$ more dilute $\mathrm{SnO}$

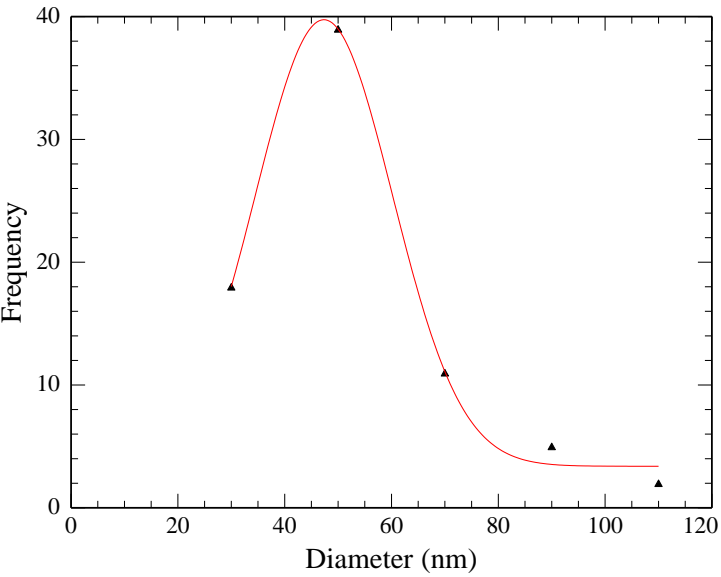

Figure B2: $\mathrm{SnO}_{2} \mathrm{NWs}$ synthesized from $\mathrm{SnO}_{\mathrm{x}} \mathrm{NPs}$ at $490^{\circ} \mathrm{C}$ in air environment without 1,10 phenanthroline along with Gaussian distributions of length and diameter. In this synthetic condition, the ratio between precursors $\mathrm{SnO}_{\mathrm{x}} \mathrm{NPs}$ and mixture of $\mathrm{LiCl} / \mathrm{KCl}$ was decreased 10 times $\left(0.02 \mathrm{~g}\right.$ of $\mathrm{SnO}_{\mathrm{x}} \mathrm{NPs}$ was mixed with $0.31 \mathrm{~g}$ of $\mathrm{LiCl}$ and $0.6 \mathrm{~g}$ of $\mathrm{KCl}$, see method $\mathrm{C}$, section 2.7.1.1) 


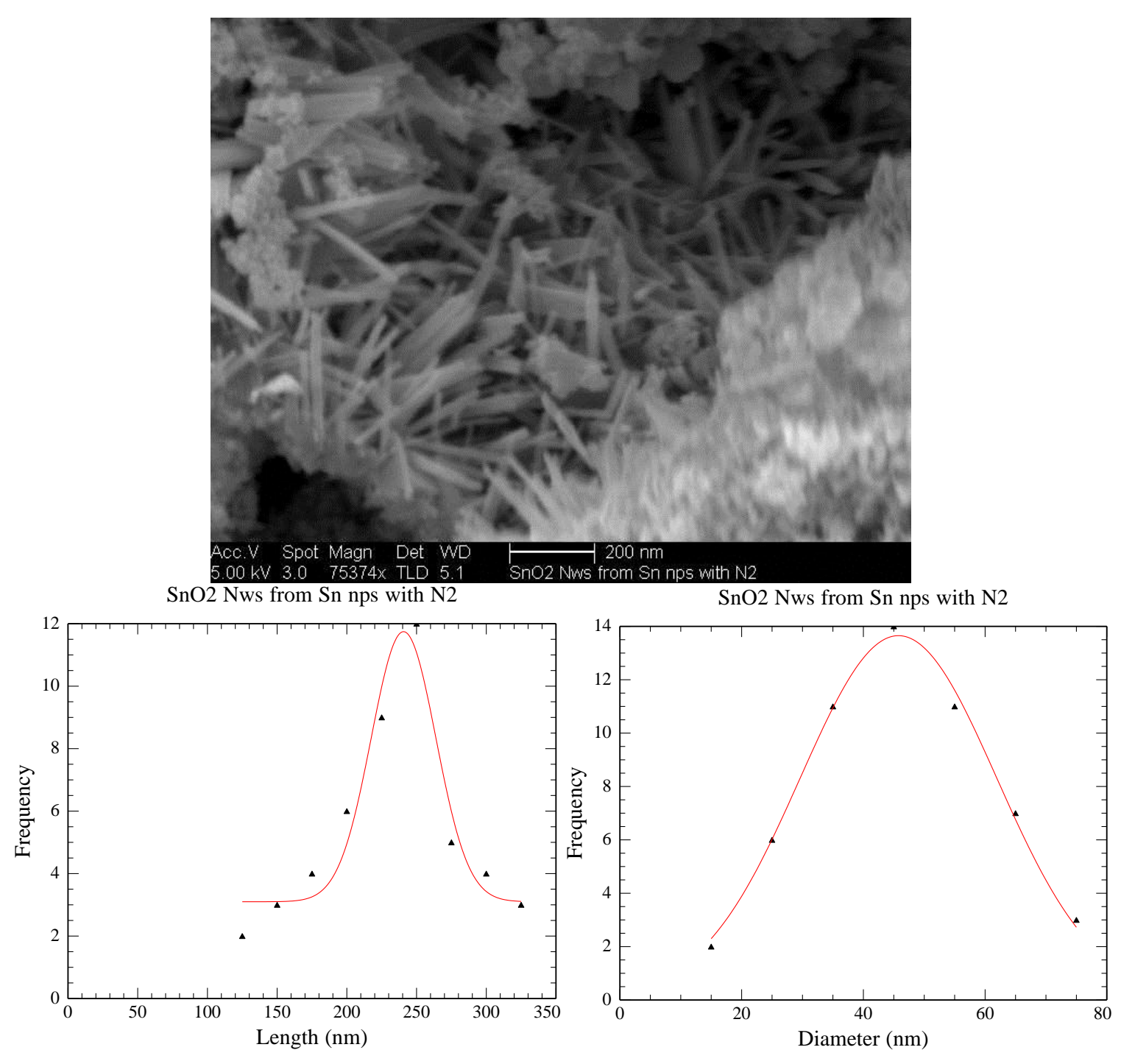

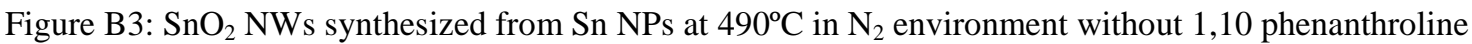
along with Gaussian distributions of length and diameter 

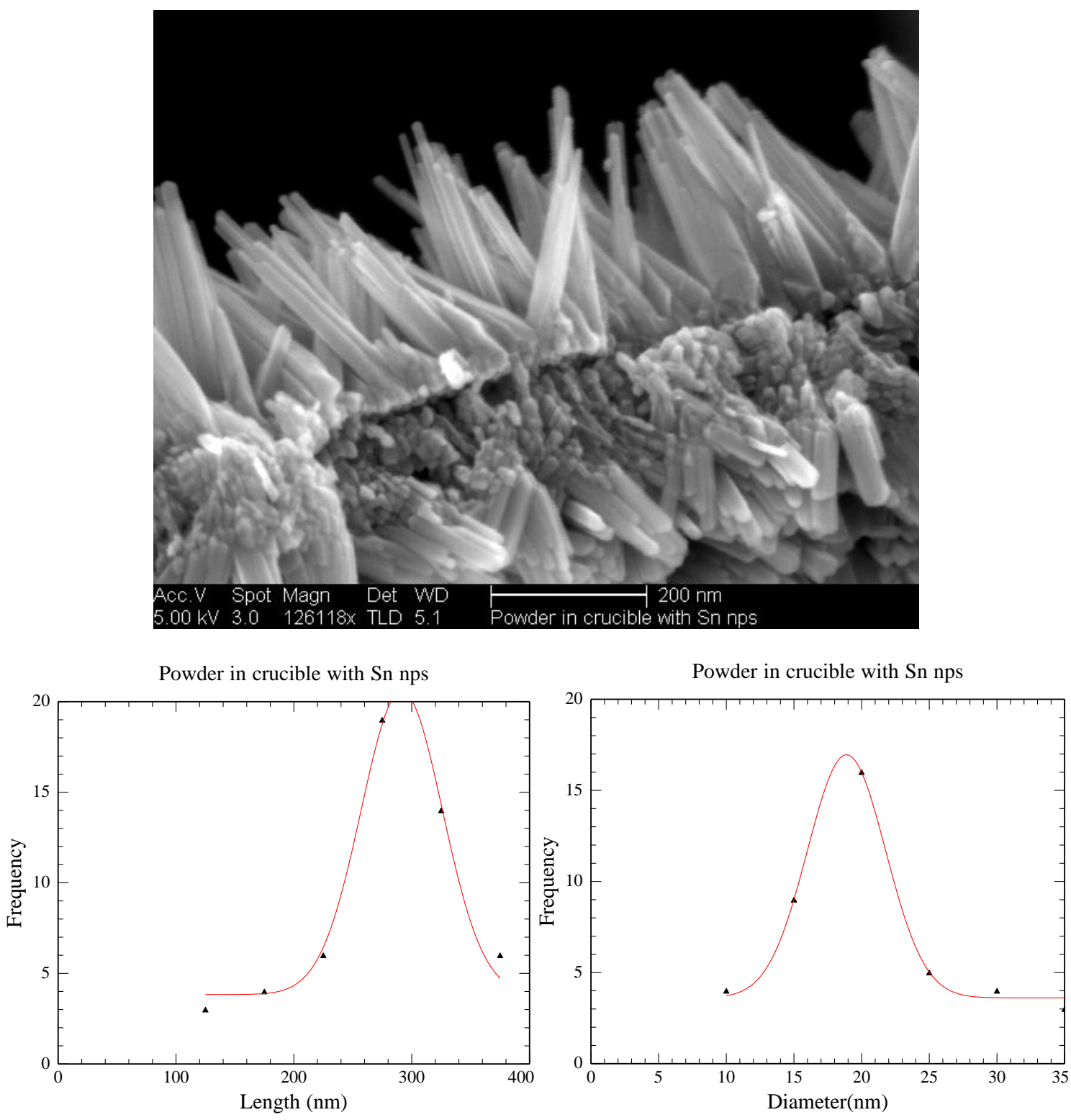

Figure B4: $\mathrm{SnO}_{2} \mathrm{NWs}$ synthesized from $\mathrm{Sn} \mathrm{NPs}$ at $490^{\circ} \mathrm{C}$ in air environment without 1,10 phenanthroline along with Gaussian distributions of length and diameter 

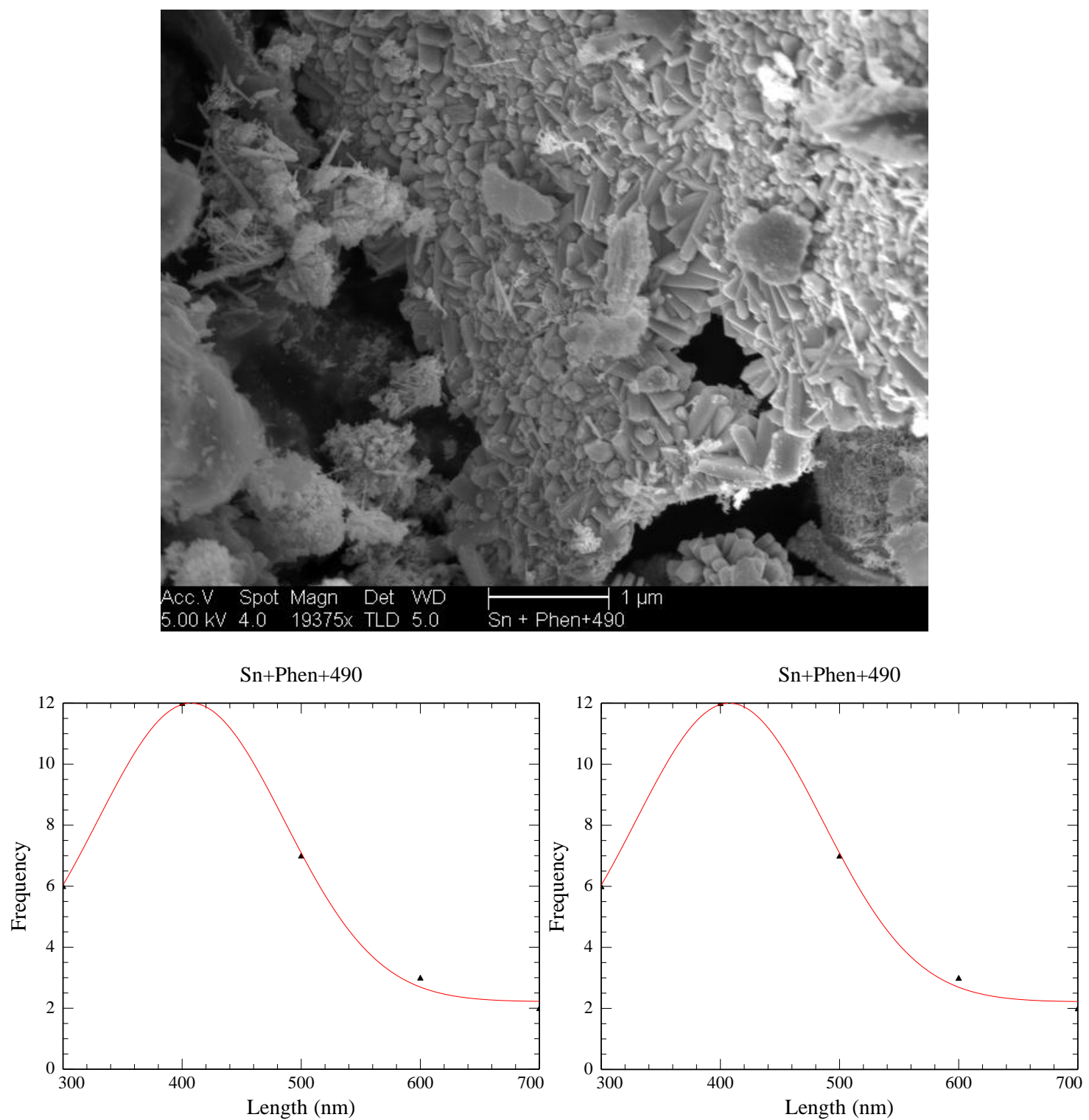

Figure B5: $\mathrm{SnO}_{2} \mathrm{NWs}$ synthesized from Sn NPs at $490^{\circ} \mathrm{C}$ in air environment with the presence of 1,10 phenanthroline along with Gaussian distributions of length and diameter 


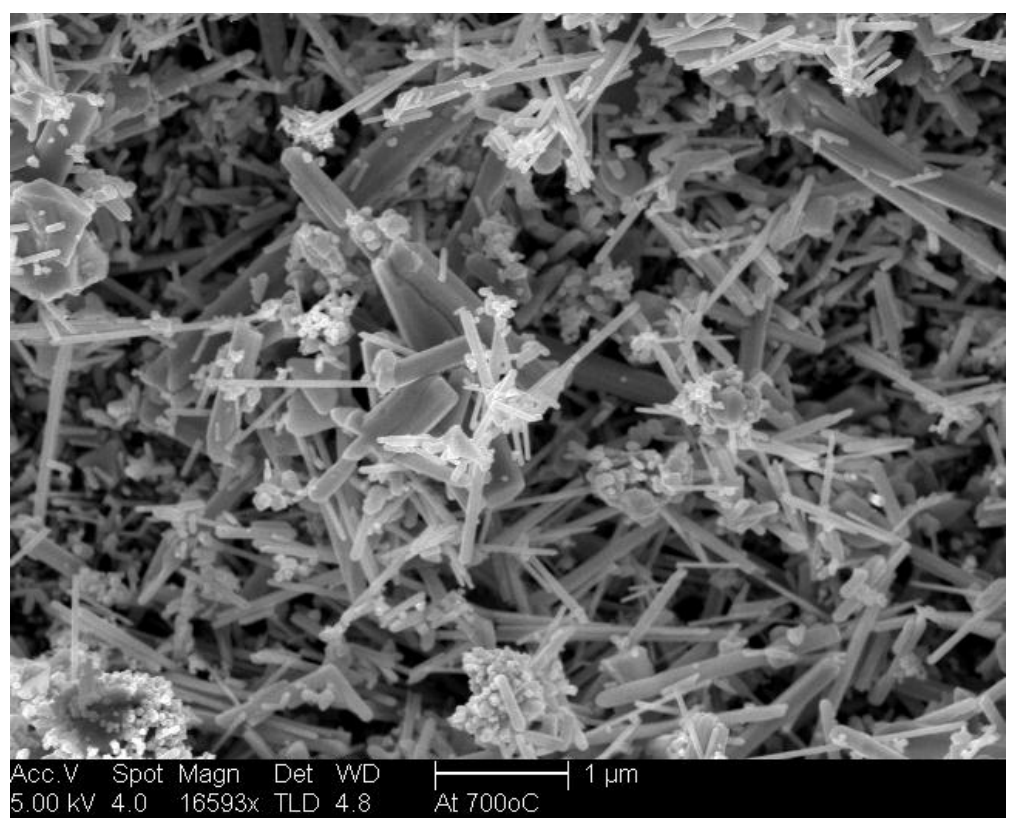

At $700 \mathrm{C}$

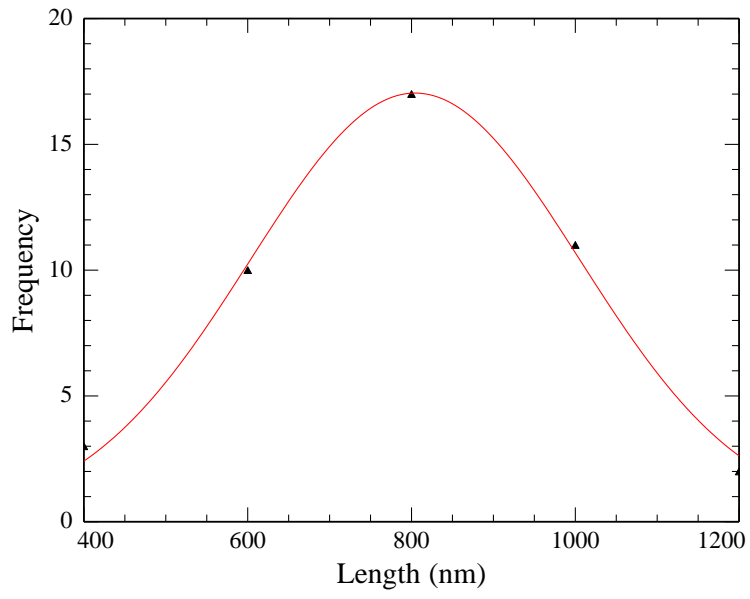

At $700 \mathrm{c}$

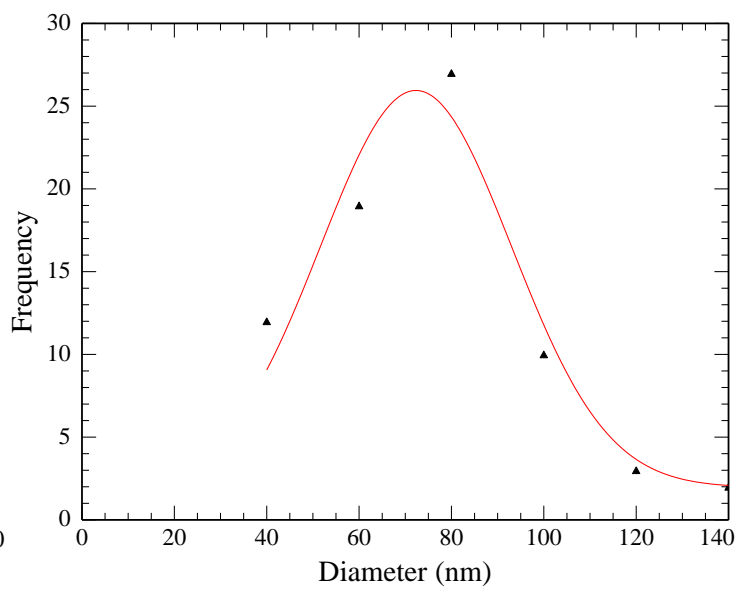

Figure B6: $\mathrm{SnO}_{2} \mathrm{NWs}$ synthesized from $\mathrm{Sn} \mathrm{NPs}$ at $700^{\circ} \mathrm{C}$ in air environment without the presence of 1,10 phenanthroline along with Gaussian distributions of length and diameter 


\section{Appendix C. Synthesis and characterization of heterojuctions of silicon nanowires}

(SiNWs) and carbon nanotubes (CNTs)

The aim of this study was to grow heterojunctions of SiNWs and CNTs using a low cost and versatile synthetic method. This includes the attachment of Au nanoparticles on functionalized MWCNTs using the ion exchange method. The hybrid structure of Au NPs/CNTs was used as a template to synthesize SiNWs, employing a novel synthetic method proposed by Chan etc (Chan, 2010 \#70). Heterostructure formation of SiNWs/CNT was characterized by electron microscopy (SEM and TEM).

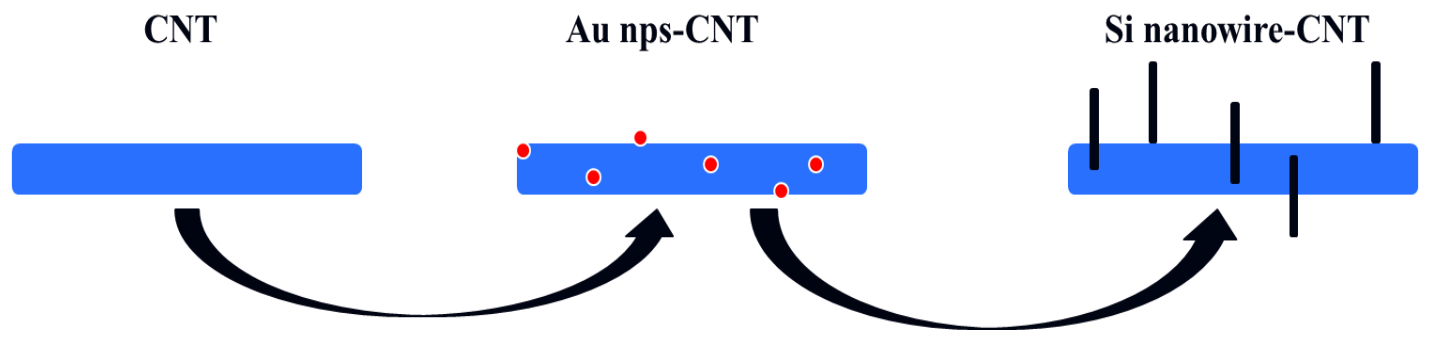

Figure C.1: Schemetic showing synthesis steps of heterostructures of SiNWs and CNTs.

\section{C.1. High density attachment of Au nanoparticcles on amine fuctionalized MWCNTs.}

0.01g of amine functionalized MWCNTs (nano-lab, catalog NO. PD30L5-20$\mathrm{NH}_{2}$ ) was dispersed in $20 \mathrm{ml} \mathrm{DI} \mathrm{H}_{2} \mathrm{O}$ by sonication and added by $0.5 \mathrm{ml}$ of Au colloid solution ( Tedpella, catalog NO. 15702-20). The mixture was stirred for 24 hours at room temperature to obtain MWCNT-Au NPs hybrid structures. The black solid was colllected and fully washed with DI water and ethanol for several times by centrifgation at 10,000 $\mathrm{rpm}$. The final black precipitate was dried at $60{ }^{\circ} \mathrm{C}$ in a vacuum oven overnight. To 
prepare the stock solution for futher syntheses, $0.001 \mathrm{~g}$ of this precipitate was dispersed in $20 \mathrm{ml}$ of water and followed by sonication.

\section{Results}

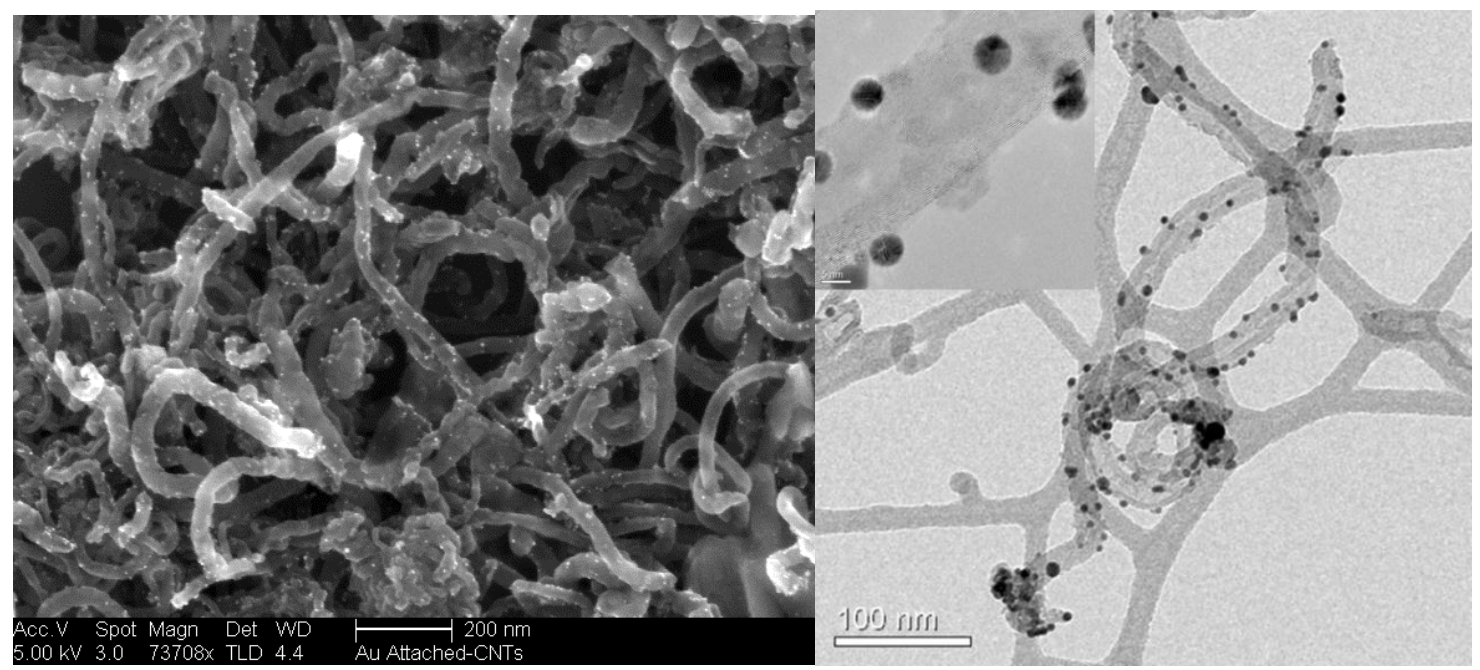

Figure C.2: SEM (left) and TEM (right) images of hybrid structure Au NPs /CNTs. The inset of TEM image show high density of Au NPs with the average diameter $5 \mathrm{~nm}$ attached on CNTs.

\section{C.2. SiNW/CNT heterojunction growth}

Au NPs attached MWCNTs were used as a template to grow heterostructures of CNT and SiNW. The synthesis was carried out using the dual chamber synthetic method (Chan, 2008 \#142). One drop of the above MWCNT-Au NPs stock solution was pipetted on a silicon substrate, followed by heating at $100{ }^{\circ} \mathrm{C}$ to completely evaporate the solvent. The substrate was inserted into the dual chamber glass reaction tube, which contains $7 \mu \mathrm{L}$ of diphenyl silane at the bottom. The tube was then sealed with a propane torch to form an approximate $13 \mathrm{~cm}$ reaction tube. Similarly, the reaction was carried out at $500{ }^{\circ} \mathrm{C}$, using a custom-built glass furnace made from heating tape.

Resutls 
Figure C.3 shows a SEM image of the as grown heterojuctions of SiNWs/CNTs. It also can be seen that a high density of SiNW growing from the sides of CNTs with Au NPs still attached on nanowire tips. Further characterization of heterostructure was obtained by HRTEM shown in Figure C.3 (right). Form this, lattice fringes of SiNWs can be observed, confirming the formation of single crystalline SiNWs. More importantly, from the inset of Figure C.3 (right), it reveals the nucleation of heterojunctions of the hybrid structure, where SiNW with Au NP on the tip nucleated from the wall of CNT.

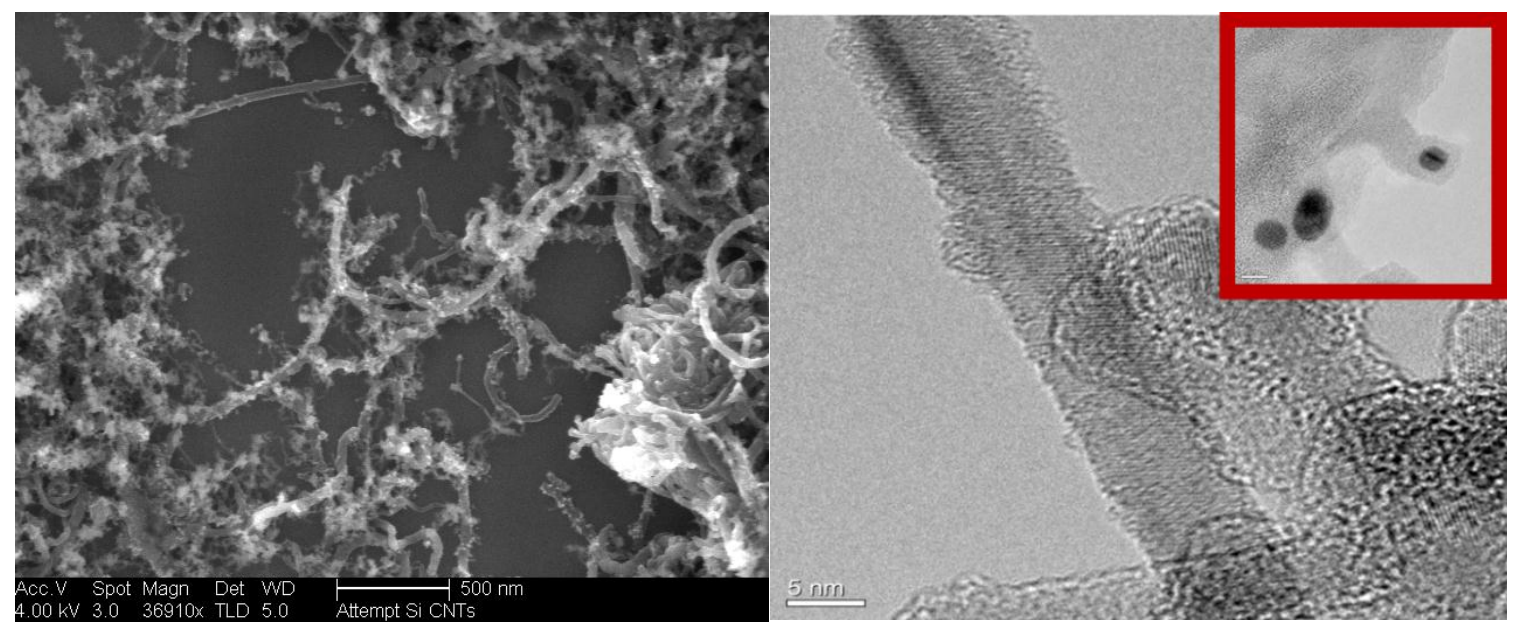

Figure C.3: SEM (left) and HRTEM (right) reveal the heterojunctions of SiNWs and CNTs 


\section{Appendix D. Negative differential resistance observed from Pd NPs attached CNTs.}

Further characterization of abnormal response behavior of the sensor toward $\mathrm{H}_{2}$ gas was carried out using the I-V characteristic. The sensor current was monitored as a function of applied voltage during $\mathrm{H}_{2}$ gas exposure process. In the experiment, the bias was applied and current was collected after introducing $\mathrm{H}_{2}$ gas into sensor testing system.

Results

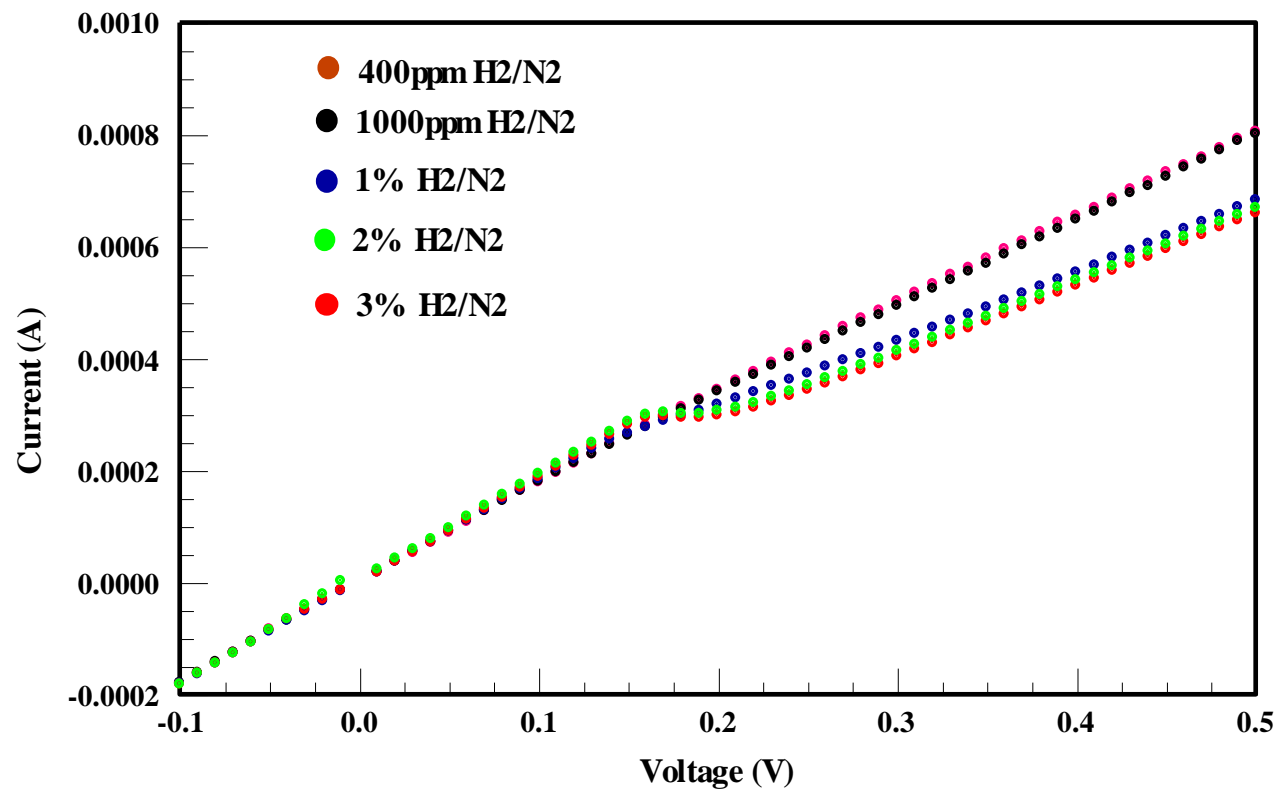

Figure D.1: Raw data of I-V curves showing abnormal behavior at different $\mathrm{H}_{2}$ concentration exposures.

The current from raw data in Figure D.1 was normalized as:

$$
I^{\prime}=\frac{V}{\frac{V}{I}-\alpha R_{0}}
$$

Where

I' is the normalized current

$\alpha$ is the normalization coefficient 
$\mathrm{R}_{0}$ is sensor resistance in air

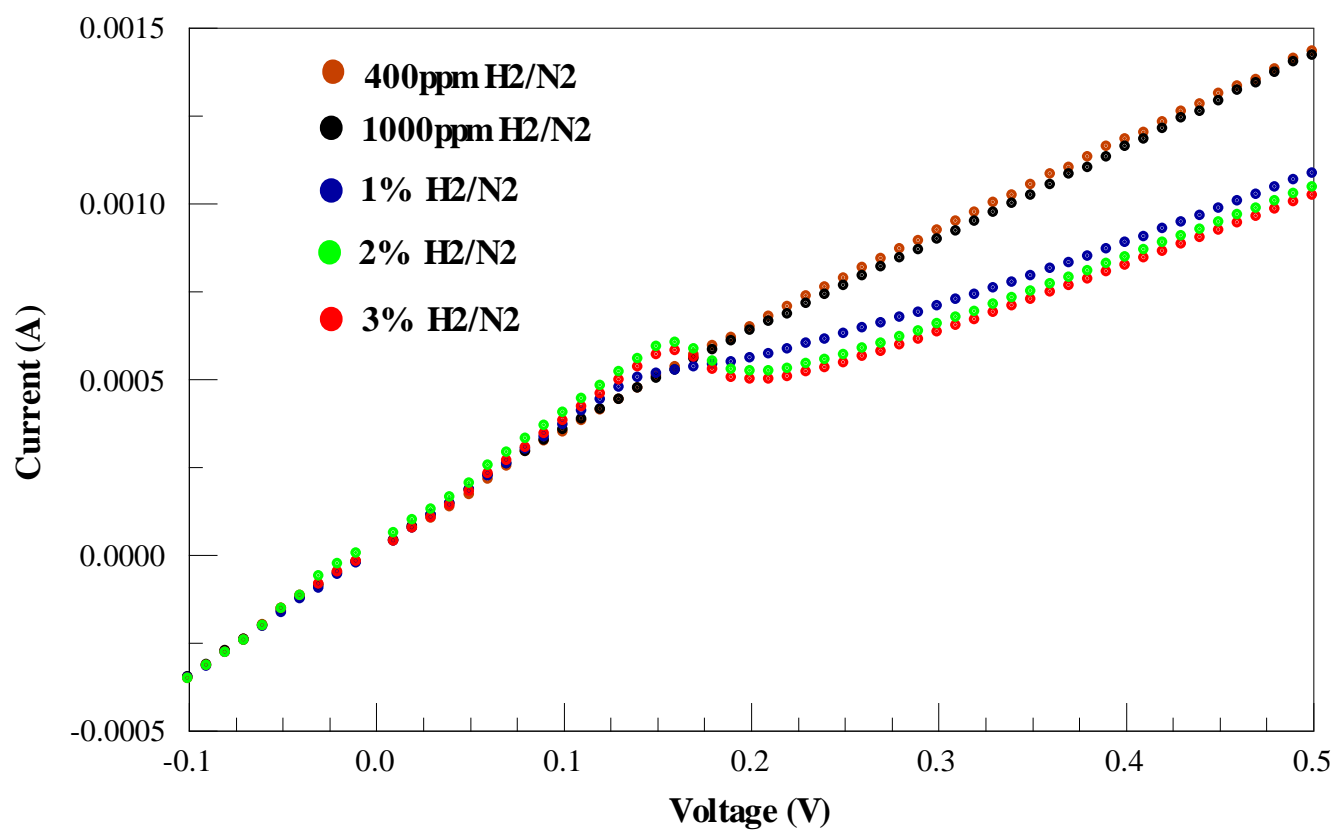

Figure D.2: Normalization of I'-V curves from Figure D.1 where $\alpha=0.95$.

Figure D.2 shows the sensor current reading at different $\mathrm{H}_{2}$ gas concentration exposures when the bias was applied from $-0.1 \mathrm{~V}$ to $0.5 \mathrm{~V}$ at the rate of $0.01 \mathrm{~V} / \mathrm{s}$. The sensor current experienced three different responses throughout such an applied voltage range. From $-0.1 \mathrm{~V}$ to $\sim 0.17 \mathrm{~V}$, Ohmic resistance of the sensor was achieved. However, when the bias reached $0.17 \mathrm{~V}$, negative differential resistance-like behavior was observed. At this point, the voltage across and the current through the sensor changed in opposite direction. An increase in voltage from $0.17 \mathrm{~V}$ to $0.22 \mathrm{~V}$ caused a decrease in current from $\sim 0.6 \mathrm{~mA}$ to $\sim 0.48 \mathrm{~mA}$. After $0.22 \mathrm{~V}$, normal Ohmic characteristic of sensor resistance was recovered. It also can be seen from Figure D.2, such behavior is more noticeable at high $\mathrm{H}_{2}$ concentration exposures, especially above $1 \%$ where charge carrier inversion was observed (Chapter 4, section 4.4.2). 
It was believed that this phenomenon is similar to negative differential resistance (NDR), which is a characteristic of a tunnel diode. Therefore, the data analysis was based on the S.M Sze model, by which the NDR region was focused and analyzed similarly to

Figure D.3 below:

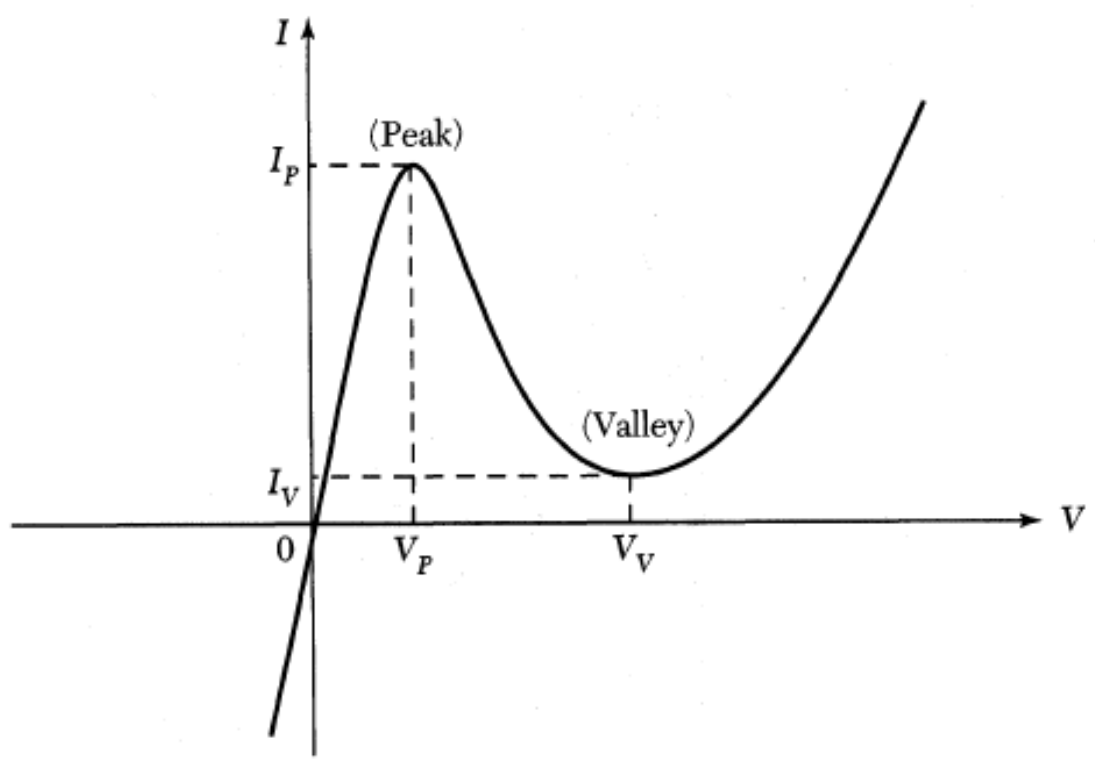

Figure D.3: Current-voltage characteristic of NDR behavior. $\mathrm{I}_{\mathrm{P}}$ and $\mathrm{V}_{\mathrm{P}}$ are the peak current and peak voltage, respectively. $I_{V}$ and $V_{V}$ are the valley current and valley voltage, respectively. 


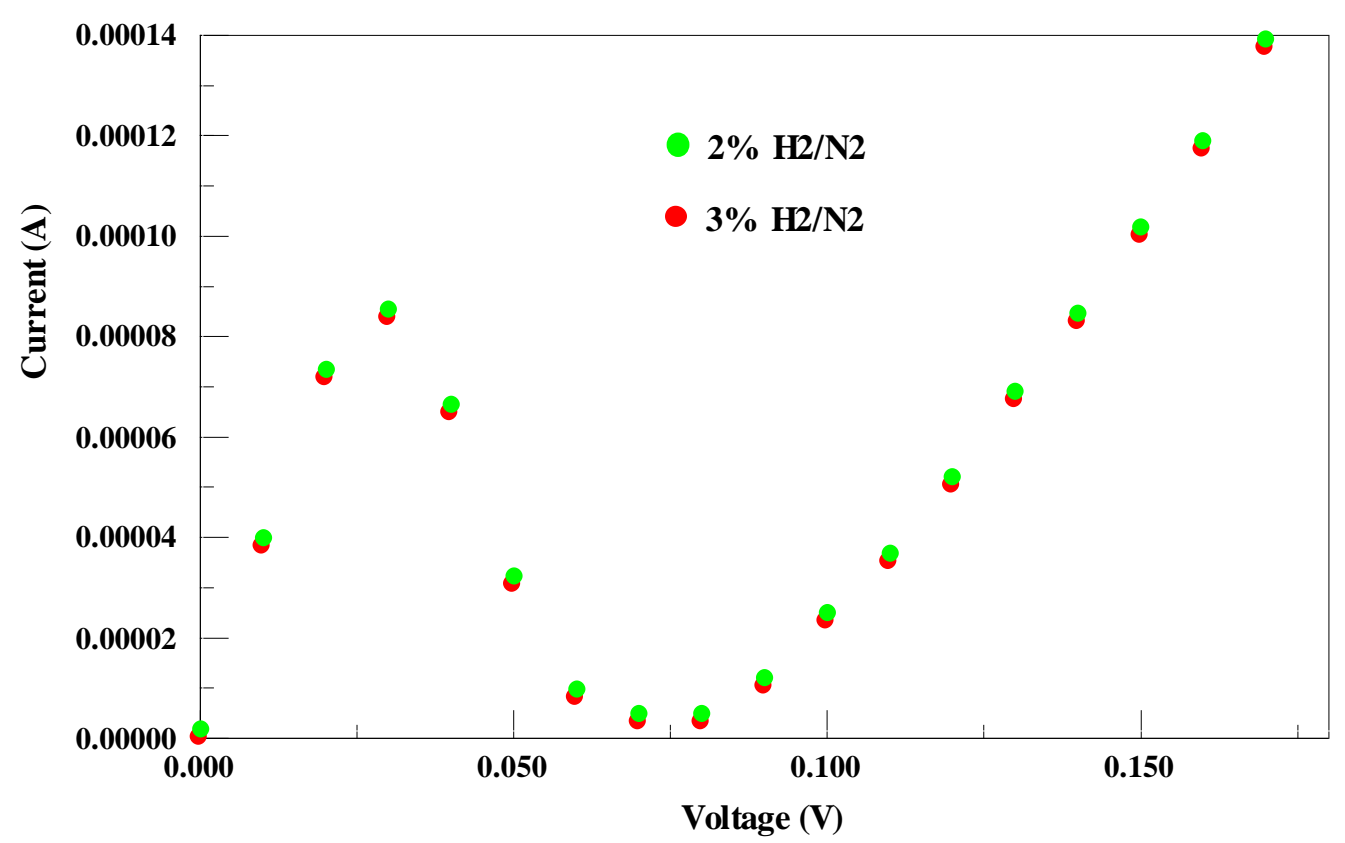

Figure D.3: Experimental I-V curves retrieved from Figure D.2. The curve data from $2 \%$ and $3 \% \mathrm{H}_{2}$ exposures were achieved from NDR-like behavior region and normalized by shifting to X0Y coordinate.

According to the S.M Sze model, the total current in negative differential

resistance behavior is the sum of tunneling, excess and diffusion currents. These currents are represented by following equations:

$$
\begin{gathered}
I_{\text {tunnel }}=I_{P}\left(\frac{V}{V_{P}}\right) \exp \left(1-\frac{V}{V_{P}}\right) \\
I_{\text {excess }}=I_{0} \exp \left(\frac{q}{K T} V\right) \\
I_{\text {diffusion }}=I_{V} \exp \left(B\left(V-V_{V}\right)\right)
\end{gathered}
$$

Where $I_{P}, I$ are the peak and valley current peak. $V_{P}$ and $V_{V}$ are the peak and valley voltage. $\mathrm{I}_{0}$ and $\mathrm{B}$ are the excess current constant and diffusion current coefficient, respectively.

Therefore: 


$$
I_{\text {Total }}=I_{P}\left(\frac{V}{V_{P}}\right) \exp \left(1-\frac{V}{V_{P}}\right)+I_{0} \exp \left(\frac{q}{K T} V\right)+I_{V} \exp \left(B_{2}\left(V-V_{V}\right)\right.
$$

Table D.1: Fitting parameters following equation D.1

\begin{tabular}{|c|c|}
\hline Fitting parameter & Value \\
\hline $\mathrm{I}_{\mathrm{P}}$ & $0.07(\mathrm{~mA})$ \\
\hline $\mathrm{V}_{\mathrm{P}}$ & $25 \mathrm{mV}$ \\
\hline $\mathrm{I}_{\mathrm{V}}$ & $0.005(\mathrm{~mA})$ \\
\hline $\mathrm{V}_{\mathrm{V}}$ & $75(\mathrm{mV})$ \\
\hline $\mathrm{B}$ & $31.2\left(\frac{1}{\mathrm{~V}}\right)$ \\
\hline $\mathrm{I}_{0}$ & $78 \mathrm{aA}$ \\
\hline $\mathrm{KT}$ & $25.8 \mathrm{eV}$ \\
\hline
\end{tabular}

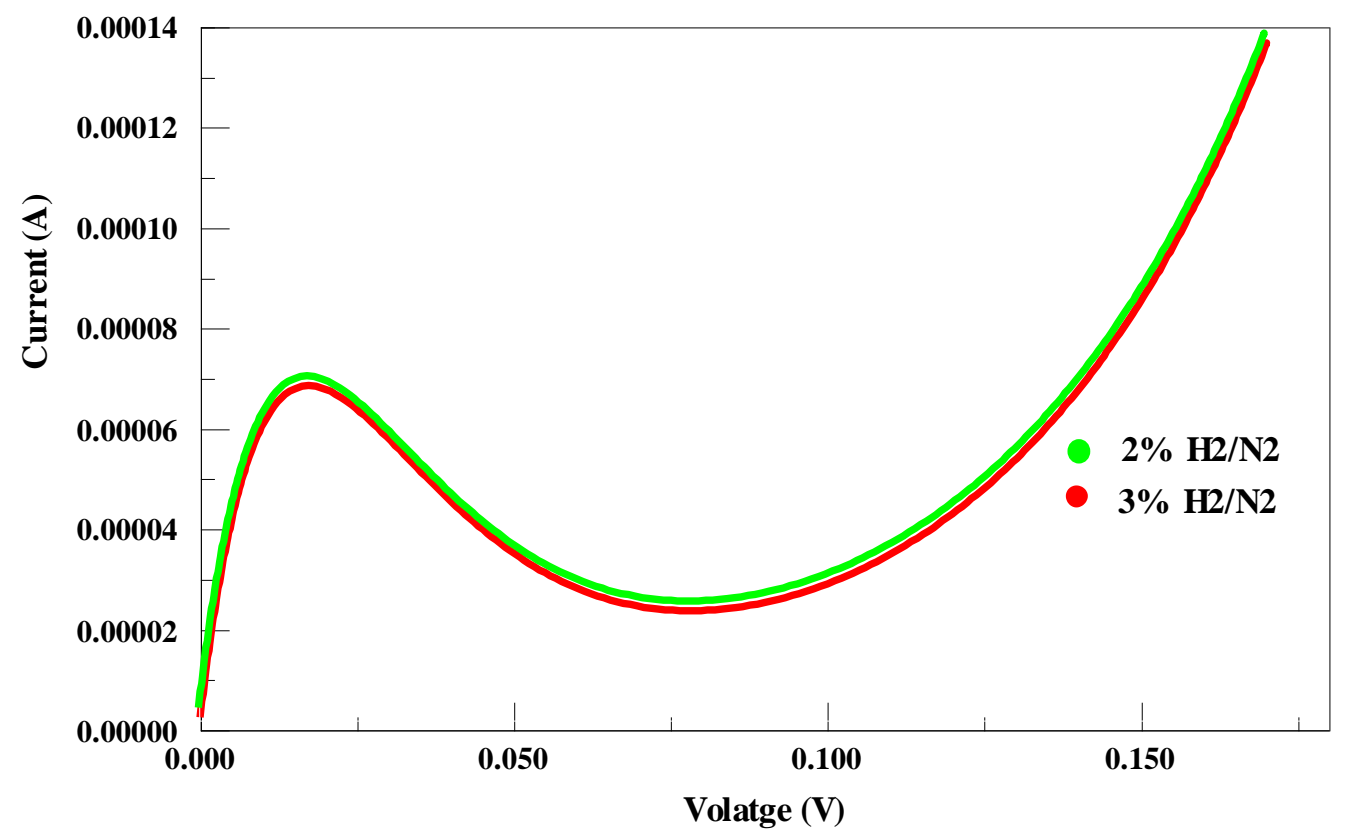

Figure D.4: I'-V curve from fitted data following eq. D.1. NDR-like behavior was obseved with $2 \%$ and 3\% $\mathrm{H}_{2}$ concetration exposures.

It was believed that such observed NDR-like behavior relates to the formation of p-n junctions, derived from charge carrier inversion. As discussed in chapter 4, under high $\mathrm{H}_{2}$ concentration exposure and the $\mathrm{H}_{2}$ molecule splitting process by Pd NPs, MWCNTs are converted from p-type to n-type semiconducting material, with respect to their outermost layers. In addition to the formation of a depletion layer, p-type 
semiconducting layers still remain inside the tube core. As a result, a single MWCNT is separated into three portions, which play a role as a p-n junction of a tunnel diode.
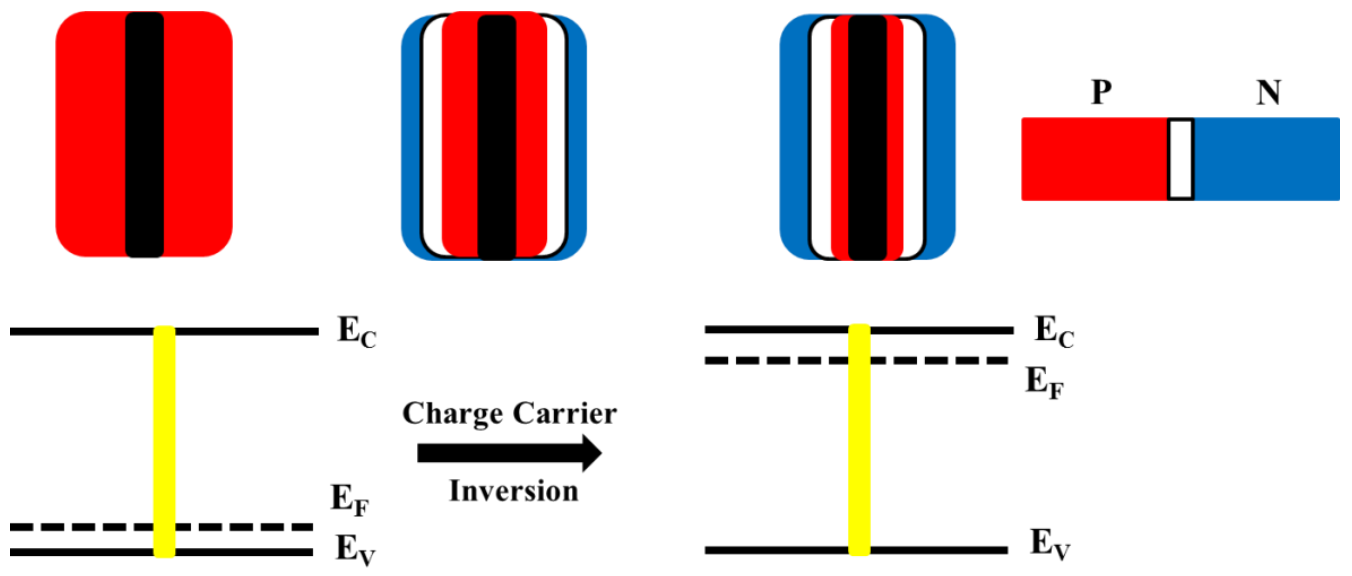

Inversion

Figure D.5: Model illustrating formation of a p-n junction in a single MWCNT along with the tube outermost layer band diagrams. The tube converted from p-type to n-type semiconducting material with respect to the outermost layers, but still remains the p-type portion inside the core. The generation of a depletion layer leads to the formation of a p-n junction.

As can be seen from the model above, both $\mathrm{p}$ and $\mathrm{n}$ sides are considered to be degenerate. When a bias is applied the electrons can tunnel from the n-side to the other due to available unoccupied states in p-side. This causes the increase of current and decrease of unoccupied state concentration. When reaching the peak value at $I_{P}$, if the voltage is further increased, there are fewer available unoccupied states on the p-side leading to the current decrease, and eventually this tunnel current stops flowing. As still increasing the bias, the normal thermal current will flow along with diffusion current. 

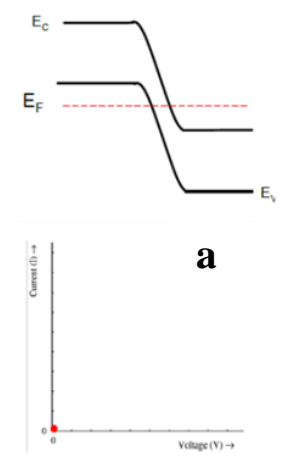
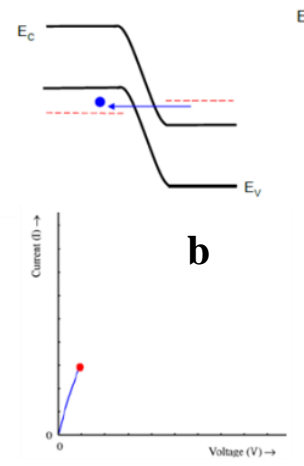
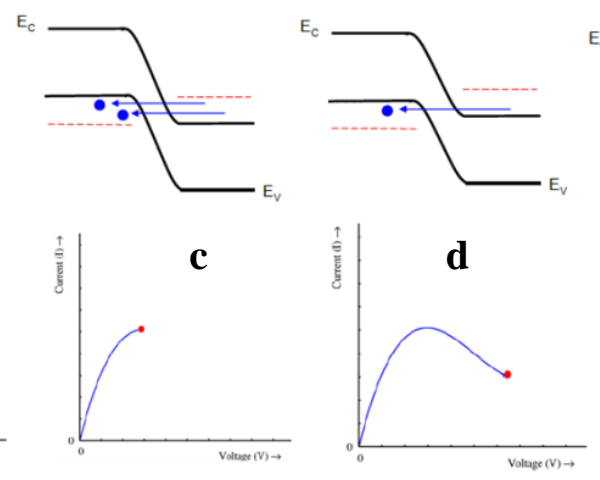
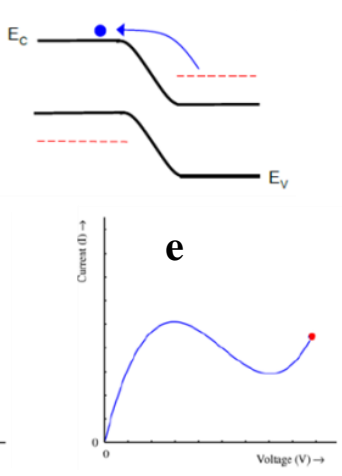

Figure D.6. Band diagram of a tunnel diode with $\mathrm{E}_{\mathrm{C}}, \mathrm{E}_{\mathrm{V}}$ and $\mathrm{E}_{\mathrm{F}}$ are the conduction band, valance band and Fermi level, respectively.

a) At zero bias, there is no current flow

b) With small bias applied, electrons in the conduction band of the n-side tunnel to available states of the valance band in the p-side

c) Increasing the voltage leads to maximum tunnel current, corresponding to a majority of electron in the n-side equal to that of available states in the p-side

d) Further increasing bias, the current decreases due to the number of electrons in the n-side decreasing

e) As higher voltage is applied, the tunnel current drops to zero. Regular diode current (thermal and diffusion currents) takes place. 